Cochrane Database of Systematic Reviews

\title{
Home-based chemically-induced whitening (bleaching) of teeth in adults (Review)
}

Eachempati P, Kumbargere Nagraj S, Kiran Kumar Krishanappa S, Gupta P, Yaylali IE

Eachempati P, Kumbargere Nagraj S, Kiran Kumar Krishanappa S, Gupta P, Yaylali IE.

Home-based chemically-induced whitening (bleaching) of teeth in adults.

Cochrane Database of Systematic Reviews 2018, Issue 12. Art. No.: CD006202.

DOI: 10.1002/14651858.CD006202.pub2.

www.cochranelibrary.com 
TABLE OF CONTENTS

HEADER

ABSTRACT

PLAIN LANGUAGE SUMMARY

SUMMARY OF FINDINGS

BACKGROUND

OBJECTIVES

METHODS

RESULTS

Figure 1.

Figure 2.

Figure 3.

Figure 4.

Figure 5.

Figure 6.

DISCUSSION

AUTHORS' CONCLUSIONS

ACKNOWLEDGEMENTS

REFERENCES

CHARACTERISTICS OF STUDIES

DATA AND ANALYSES

Analysis 1.1. Comparison $1 \mathrm{CP}$ gel in tray versus placebo, Outcome 1 Tooth whitening - assessed by the dentist.

Analysis 2.1. Comparison $2 \mathrm{CP}$ gel in tray versus placebo, Outcome 1 Tooth whitening - assessed by the dentist.

Analysis 3.1. Comparison $3 \mathrm{HP}$ gel in tray versus placebo, Outcome 1 Tooth whitening - assessed by the dentist.

Analysis 4.1. Comparison 4 HP strip versus placebo, Outcome 1 Tooth whitening - assessed by the dentist.

Analysis 5.1. Comparison 5 CP paint-on gel versus placebo, Outcome 1 Tooth whitening - assessed by the dentist.

Analysis 6.1. Comparison 6 HP paint-on gel versus placebo, Outcome 1 Tooth whitening - assessed by the dentist.

Analysis 7.1. Comparison 7 Chewing gum SHMP versus placebo, Outcome 1 Tooth whitening - assessed by the dentist. ..........

Analysis 8.1. Comparison 8 Chewing gum STPP versus placebo, Outcome 1 Tooth whitening - assessed by the dentist. ..........

Analysis 9.1. Comparison 9 HP mouthwash versus placebo, Outcome 1 Tooth whitening - assessed by the dentist.

Analysis 10.1. Comparison $10 \mathrm{CP}$ tray versus CP tray, Outcome 1 Tooth whitening - assessed by the dentist.

Analysis 11.1. Comparison $11 \mathrm{CP}$ tray versus CP tray, Outcome 1 Tooth whitening - assessed by the dentist.

Analysis 11.2. Comparison $11 \mathrm{CP}$ tray versus CP tray, Outcome 2 Tooth whitening - assessed by the dentist.

Analysis 11.3. Comparison $11 \mathrm{CP}$ tray versus CP tray, Outcome 3 Tooth whitening - reported by the patient.

Analysis 12.1. Comparison $12 \mathrm{CP}$ tray versus CP tray, Outcome 1 Tooth whitening - assessed by the dentist.

Analysis 12.2. Comparison $12 \mathrm{CP}$ tray versus CP tray, Outcome 2 Tooth whitening - assessed by the dentist.

Analysis 12.3. Comparison 12 CP tray versus CP tray, Outcome 3 Tooth whitening - assessed by the dentist.

Analysis 13.1. Comparison $13 \mathrm{CP}$ tray versus HP tray, Outcome 1 Tooth whitening - assessed by the dentist.

Analysis 13.2. Comparison $13 \mathrm{CP}$ tray versus HP tray, Outcome 2 Tooth whitening - assessed by the dentist.

Analysis 14.1. Comparison $14 \mathrm{HP}$ strip versus CP tray, Outcome 1 Tooth whitening - assessed by the dentist.

Analysis 14.2. Comparison $14 \mathrm{HP}$ strip versus CP tray, Outcome 2 Tooth whitening - assessed by the dentist.

Analysis 14.3. Comparison $14 \mathrm{HP}$ strip versus CP tray, Outcome 3 Tooth whitening - reported by the patient.

Analysis 15.1. Comparison 15 HP strip versus HP tray, Outcome 1 Tooth whitening - assessed by the dentist.

Analysis 15.2. Comparison 15 HP strip versus HP tray, Outcome 2 Patient comfort.

Analysis 16.1. Comparison 16 HP strip versus HP strip, Outcome 1 Tooth whitening - assessed by the dentist.

Analysis 17.1. Comparison 17 HP strip versus HP mouthwash, Outcome 1 Tooth whitening - assessed by the dentist.

Analysis 18.1. Comparison $18 \mathrm{CP}$ paint-on versus HP strip, Outcome 1 Tooth whitening - assessed by the dentist.

Analysis 19.1. Comparison 19 HP paint-on versus HP strip, Outcome 1 Tooth whitening - assessed by the dentist.

Analysis 19.2. Comparison 19 HP paint-on versus HP strip, Outcome 2 Tooth whitening - assessed by the dentist.

Analysis 19.3. Comparison 19 HP paint-on versus HP strip, Outcome 3 Tooth whitening - reported by the patient. 
Analysis 22.1. Comparison $22 \mathrm{CP}$ paint-on versus HP paint-on, Outcome 1 Tooth whitening - assessed by the dentist.

Analysis 23.1. Comparison 23 HP paint-on versus HP paint-on, Outcome 1 Tooth whitening - assessed by the dentist.

Analysis 24.1. Comparison 24 SPC paint-on versus CP paint-on, Outcome 1 Tooth whitening - assessed by the dentist.

Analysis 25.1. Comparison 25 SPC paint-on versus HP paint-on, Outcome 1 Tooth whitening - assessed by the dentist.

WHAT'S NEW

DECLARATIONS OF INTEREST

SOURCES OF SUPPORT 
[Intervention Review]

\section{Home-based chemically-induced whitening (bleaching) of teeth in adults}

Prashanti Eachempati¹, Sumanth Kumbargere Nagraj², Salian Kiran Kumar Krishanappa1, Puneet Gupta ${ }^{3}$, Ibrahim Ethem Yaylali4

1Department of Prosthodontics, Faculty of Dentistry, Melaka-Manipal Medical College, Manipal Academy of Higher Education (MAHE), Melaka, Malaysia. 2Department of Oral Medicine and Oral Radiology, Faculty of Dentistry, Melaka-Manipal Medical College, Manipal Academy of Higher Education (MAHE), Manipal, Melaka, Malaysia. 3Public Health Dentistry, Government College of Dentistry, Indore, Indore, India. ${ }^{4}$ Dentistry, Endodontics, Isparta Military Hospital, Isparta, Turkey

Contact address: Prashanti Eachempati, Department of Prosthodontics, Faculty of Dentistry, Melaka-Manipal Medical College, Manipal Academy of Higher Education (MAHE), Jalan Batu Hampar, Bukit Baru, Melaka, 75150, Malaysia. prashanti.eachempati@manipal.edu.my.

Editorial group: Cochrane Oral Health Group.

Publication status and date: New search for studies and content updated (conclusions changed), published in Issue 12, 2018.

Citation: Eachempati P, Kumbargere Nagraj S, Kiran Kumar Krishanappa S, Gupta P, Yaylali IE. Home-based chemically-induced whitening (bleaching) of teeth in adults. Cochrane Database of Systematic Reviews 2018, Issue 12. Art. No.: CD006202. DOI: 10.1002/14651858.CD006202.pub2.

Copyright @ 2018 The Cochrane Collaboration. Published by John Wiley \& Sons, Ltd.

\section{A B S T R A C T}

\section{Background}

With the increased demand for whiter teeth, home-based bleaching products, either dentist-prescribed or over-the-counter products have been exponentially increasing in the past few decades. This is an update of a Cochrane Review first published in 2006.

\section{Objectives}

To evaluate the effects of home-based tooth whitening products with chemical bleaching action, dispensed by a dentist or over-thecounter.

\section{Search methods}

Cochrane Oral Health's Information Specialist searched the following databases: Cochrane Oral Health's Trials Register (to 12 June 2018), the Cochrane Central Register of Controlled Trials (CENTRAL; 2018, Issue 6) in the Cochrane Library (searched 12 June 2018), MEDLINE Ovid (1946 to 12 June 2018), and Embase Ovid (1980 to 12 June 2018). The US National Institutes of Health Ongoing Trials Register ClinicalTrials.gov (12 June 2018) and the World Health Organization International Clinical Trials Registry Platform (12 June 2018 ) were searched for ongoing trials. No restrictions were placed on the language or date of publication when searching the electronic databases.

\section{Selection criteria}

We included in our review randomised controlled trials (RCTs) which involved adults who were 18 years and above, and compared dentistdispensed or over-the-counter tooth whitening (bleaching) products with placebo or other comparable products.

Quasi-randomised trials, combination of in-office and home-based treatments, and home-based products having physical removal of stains were excluded.

\section{Data collection and analysis}

Two review authors independently selected trials. Two pairs of review authors independently extracted data and assessed risk of bias. We estimated risk ratios (RRs) for dichotomous data, and mean differences (MDs) or standardised mean difference (SMD) for continuous data, with $95 \%$ confidence intervals (Cls). We assessed the certainty of the evidence using the GRADE approach. 


\section{Main results}

We included 71 trials in the review with 26 studies (1398 participants) comparing a bleaching agent to placebo and 51 studies (2382 participants) comparing a bleaching agent to another bleaching agent. Two studies were at low overall risk of bias; two at high overall risk of bias; and the remaining 67 at unclear overall risk of bias.

The bleaching agents (carbamide peroxide (CP) gel in tray, hydrogen peroxide (HP) gel in tray, HP strips, CP paint-on gel, HP paint-on gel, sodium hexametaphosphate (SHMP) chewing gum, sodium tripolyphosphate (STPP) chewing gum, and HP mouthwash) at different concentrations with varying application times whitened teeth compared to placebo over a short time period (from 2 weeks to 6 months), however the certainty of the evidence is low to very low.

In trials comparing one bleaching agent to another, concentrations, application method and application times, and duration of use varied widely. Most of the comparisons were reported in single trials with small sample sizes and event rates and certainty of the evidence was assessed as low to very low. Therefore the evidence currently available is insufficient to draw reliable conclusions regarding the superiority of home-based bleaching compositions or any particular method of application or concentration or application time or duration of use.

Tooth sensitivity and oral irritation were the most common side effects which were more prevalent with higher concentrations of active agents though the effects were mild and transient. Tooth whitening did not have any effect on oral health-related quality of life.

\section{Authors' conclusions}

We found low to very low-certainty evidence over short time periods to support the effectiveness of home-based chemically-induced bleaching methods compared to placebo for all the outcomes tested.

We were unable to draw any conclusions regarding the superiority of home-based bleaching compositions or any particular method of application or concentration or application time or duration of use, as the overall evidence generated was of very low certainty. Well-planned RCTs need to be conducted by standardising methods of application, concentrations, application times, and duration of treatment.

\section{PLAIN LANGUAGE SUMMARY}

\section{Home-based chemical bleaching of teeth in adults}

\section{Review question}

What evidence is available regarding the different home-based chemically-induced bleaching agents in whitening teeth?

\section{Background}

There has been an increasing demand for whiter teeth. Home-based whitening products with a bleaching action have become popular and are prescribed to people by the dentist or purchased over-the-counter. A variety of whitening products are available which include hydrogen peroxide, carbamide peroxide, sodium percarbonate, sodium hexametaphosphate, sodium tripolyphosphate, and calcium peroxide. These agents are supplied in different concentrations and are used with different methods of application (gel in tray, strips, painton gel, chewing gum, and mouthwash), which have varying application times and duration of treatment.

\section{Study characteristics}

Authors from Cochrane Oral Health carried out this review of existing studies and the evidence is current up to 12 June 2018 . We included 71 trials that involved 3780 adults who underwent tooth whitening procedures with various bleaching agents using different methods of application, length of application and duration of treatment. 26 studies compared a bleaching agent to placebo and 51 studies compared one bleaching agent to another bleaching agent.

\section{Key results}

The bleaching agents whitened teeth compared to placebo over a short time period (from 2 weeks to 6 months), however the certainty of the evidence is low to very low.

The evidence currently available is insufficient to draw reliable conclusions regarding the superiority of home-based bleaching compositions or any particular method of application or concentration or application time or duration of use.

The most common adverse events were tooth sensitivity and oral irritation, which were reported with higher concentrations of active agents, although the effects were mild and transient.

Well-planned randomised controlled trials need to be conducted by standardising methods of application, concentrations, application times and duration of treatment.

\section{Certainty of evidence}

The overall certainty of the evidence was low to very low for all comparisons. This was because most of the comparisons were reported in single trials with small sample sizes and event rates. There was an unclear risk of bias in most of the trials. 


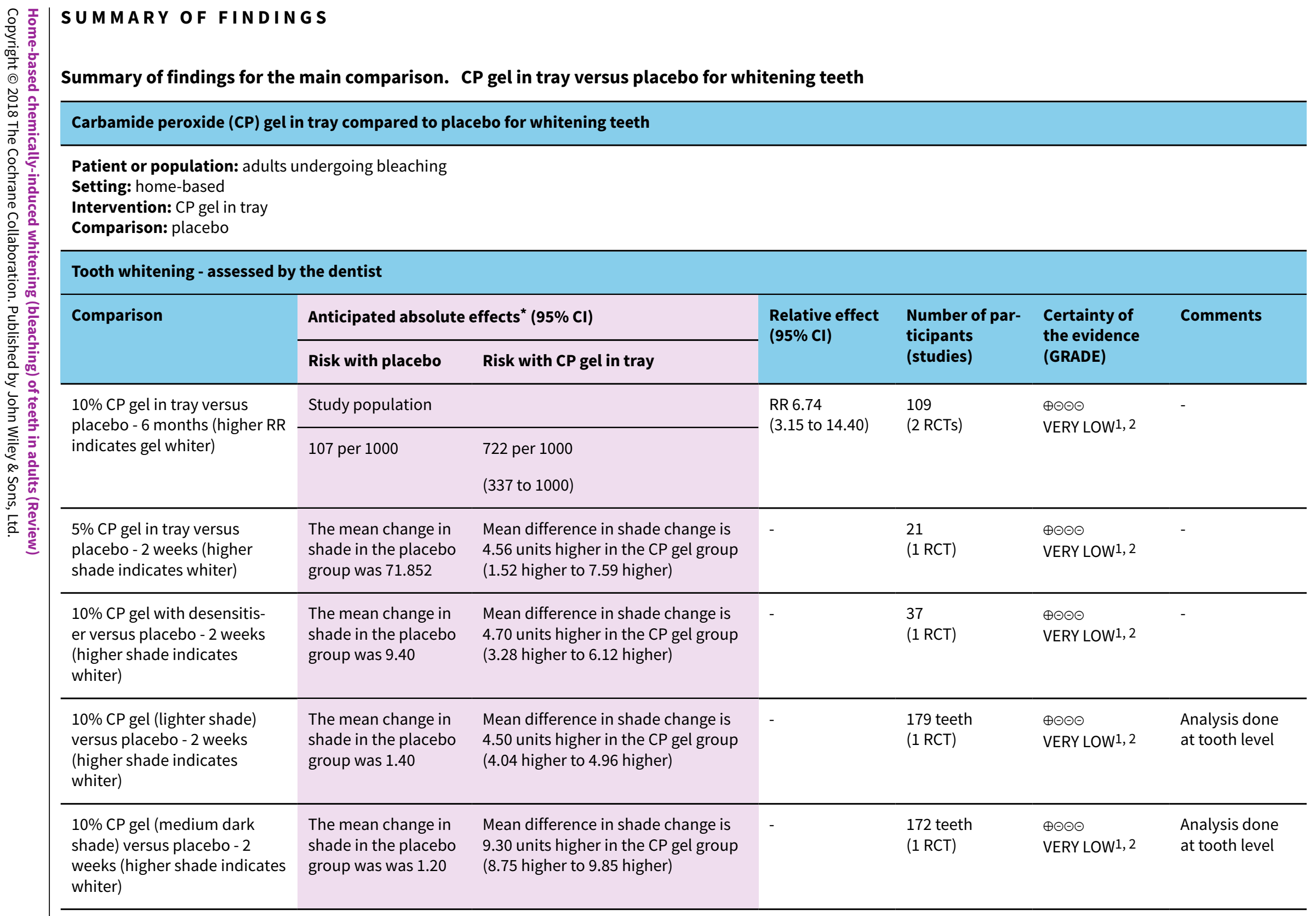


$10 \%$ CP gel (dark shade) versus placebo - 2 weeks (higher shade indicates whiter)

\section{Adverse effects}

Main adverse events reported in majority of trials were mild and transient tooth sensitivity and oral irritation which occurred more in the intervention group compared to placebo

${ }^{*}$ The risk in the intervention group (and its $95 \%$ confidence interval) is based on the assumed risk in the comparison group and the relative effect of the intervention (and its $95 \% \mathrm{Cl})$

Cl: confidence interval; $\mathbf{R C T}$ : randomised controlled trial; RR: risk ratio

\section{GRADE Working Group grades of evidence}

High certainty: we are very confident that the true effect lies close to that of the estimate of the effect

Moderate certainty: we are moderately confident in the effect estimate: the true effect is likely to be close to the estimate of the effect, but there is a possibility that it is substantially different

Low certainty: our confidence in the effect estimate is limited: the true effect may be substantially different from the estimate of the effect

Very low certainty: we have very little confidence in the effect estimate: the true effect is likely to be substantially different from the estimate of effect

1Downgraded for risk of bias - unclear risk of bias due to lack of allocation concealment, performance and detection bias.

2Downgraded for imprecision - low sample size and event rate.

\section{Summary of findings 2. HP gel in tray versus placebo for whitening teeth}

\section{Hydrogen peroxide (HP) gel in tray compared to placebo for whitening teeth}

Patient or population: adults undergoing bleaching

Setting: home-based

Intervention: HP gel in tray

Comparison: placebo

\section{Tooth whitening - assessed by the dentist}

\section{Comparison}

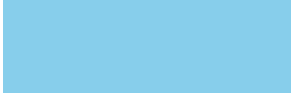

$6 \% \mathrm{HP}$ gel versus placebo - 14 days

\begin{tabular}{ll}
\hline \multicolumn{2}{l}{ Anticipated absolute effects ${ }^{\star}(\mathbf{9 5 \%} \mathrm{Cl})$} \\
\hline Risk with placebo & Risk with HP gel in tray \\
\hline $\begin{array}{l}\text { The mean change in } \\
\text { shade in placebo group } \\
\text { was 0.48 }\end{array}$ & $\begin{array}{l}\text { Mean difference in shade change is 3.08 } \\
\text { units higher in the HP gel group } \\
(2.28 \text { higher to 3.88 higher })\end{array}$ \\
\hline
\end{tabular}

Relative effect
$(95 \% \mathrm{CI})$

$(95 \% \mathrm{Cl})$

Number of par-

ticipants

(studies)

(1 RCT)

was 0.48

(2.28 higher to 3.88 higher)

$\begin{aligned} & \text { Certainty of } \\ & \text { the evidence } \\ & \text { (GRADE) }\end{aligned}$
$\begin{aligned} & \oplus \odot \odot \ominus \\ & \text { VERY LOW1, } 2\end{aligned}$

\begin{tabular}{|c|c|}
\hline $\begin{array}{l}\text { The mean change in } \\
\text { shade in the placebo }\end{array}$ & $\begin{array}{l}\text { Mean difference in shade change is } \\
10 \text { units higher in the CP gel group }\end{array}$ \\
\hline
\end{tabular}




\section{Adverse effects}

Not reported

${ }^{\star}$ The risk in the intervention group (and its $95 \%$ confidence interval) is based on the assumed risk in the comparison group and the relative effect of the intervention (and its $95 \% \mathrm{Cl}$ )

Cl: confidence interval; RCT: randomised controlled trial

\section{GRADE Working Group grades of evidence}

High certainty: we are very confident that the true effect lies close to that of the estimate of the effect

Moderate certainty: we are moderately confident in the effect estimate: the true effect is likely to be close to the estimate of the effect, but there is a possibility that it is

substantially different

Low certainty: our confidence in the effect estimate is limited: the true effect may be substantially different from the estimate of the effect

Very low certainty: we have very little confidence in the effect estimate: the true effect is likely to be substantially different from the estimate of effect

1Downgraded for risk of bias - unclear risk of bias due to selection, performance and detection bias.

2Downgraded for imprecision - single trial and low sample and event rate.

Summary of findings 3. HP strips versus placebo for whitening teeth

Hydrogen peroxide (HP) strips compared to placebo for whitening teeth

Patient or population: adults undergoing bleaching

Setting: home-based

Intervention: HP strips

Comparison: placebo

Tooth whitening - assessed by the dentist

\begin{tabular}{|c|c|c|c|c|c|c|}
\hline \multirow[t]{2}{*}{ Comparison } & \multicolumn{2}{|c|}{ Anticipated absolute effects ${ }^{\star}(95 \% \mathrm{Cl})$} & \multirow{2}{*}{$\begin{array}{l}\text { Relative effect } \\
(95 \% \mathrm{Cl})\end{array}$} & \multirow{2}{*}{$\begin{array}{l}\text { Number of par- } \\
\text { ticipants } \\
\text { (studies) }\end{array}$} & \multirow{2}{*}{$\begin{array}{l}\text { Certainty of } \\
\text { the evidence } \\
\text { (GRADE) }\end{array}$} & \multirow[t]{2}{*}{ Comments } \\
\hline & Risk with placebo & Risk with HP strips & & & & \\
\hline $\begin{array}{l}10 \% \text { HP strip versus place- } \\
\text { bo - day } 8 \text { (higher shade in- } \\
\text { dicates whiter) }\end{array}$ & $\begin{array}{l}\text { The mean change } \\
\text { in shade in placebo } \\
\text { group was } 0.21\end{array}$ & $\begin{array}{l}\text { Mean difference in shade change is } \\
2.24 \text { units higher in the HP strip group } \\
\text { ( } 1.72 \text { higher to } 2.76 \text { higher) }\end{array}$ & - & $\begin{array}{l}36 \\
(1 \mathrm{RCT})\end{array}$ & $\begin{array}{l}\oplus \odot \odot \odot \\
\text { VERY LOW } 1,2\end{array}$ & - \\
\hline
\end{tabular}




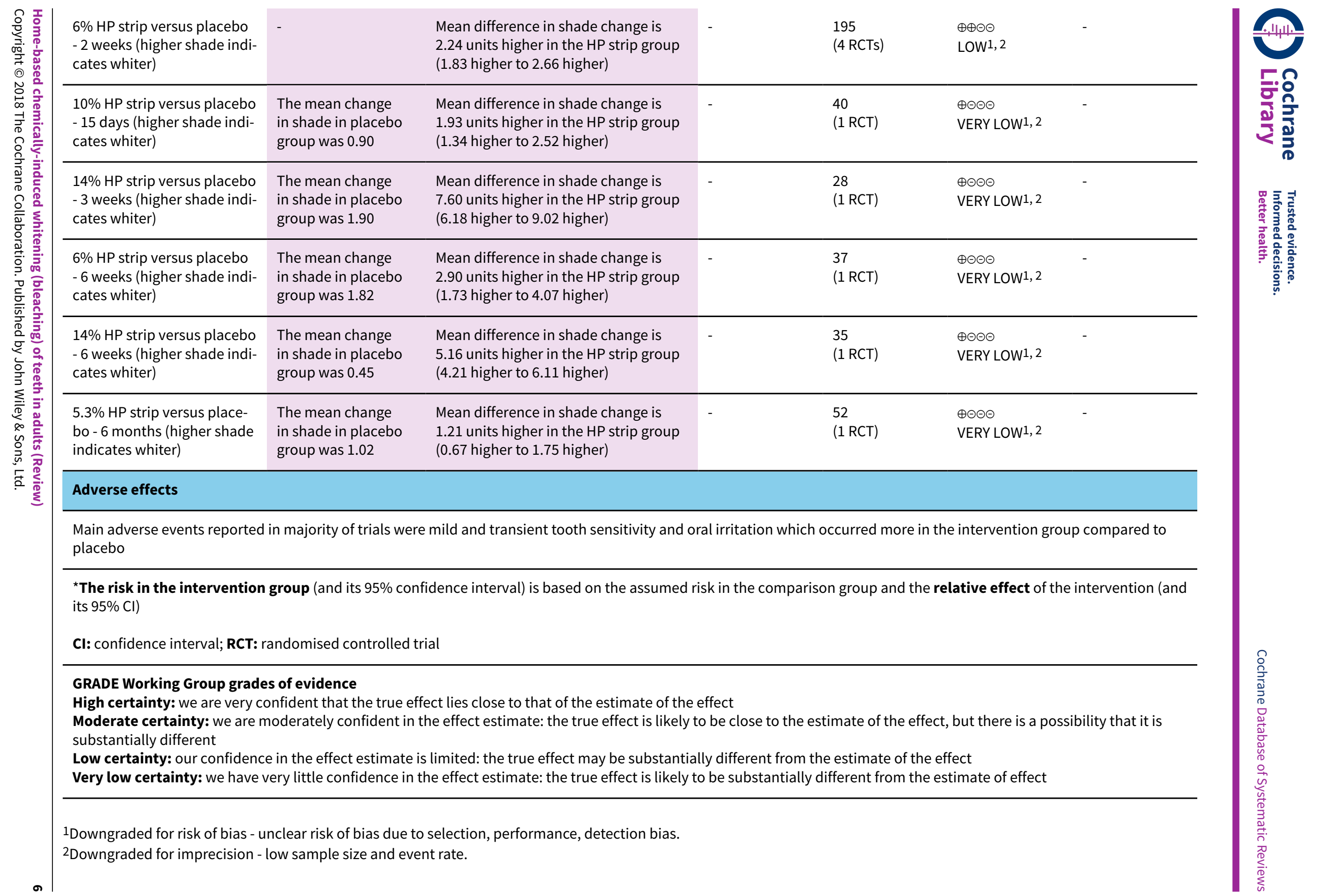




\section{Carbamide peroxide (CP) paint-on gel compared to placebo for whitening teeth}

Patient or population: adults undergoing bleaching

Setting: home-based

Intervention: $\mathrm{CP}$ paint-on ge

Comparison: placebo

\section{Tooth whitening - assessed by the dentist}

Comparisons

Anticipated absolute effects* $(95 \% \mathrm{Cl})$

Relative effect

Number of par-

Certainty of

Comments Risk with placebo

(studies)

the evidence

(GRADE)

$18 \%$ CP paint-on gel

versus placebo - 3

The mean shade

Mean difference in the shade change was

change in the placebo 3.50 higher in the CP paint-on gel group

$\oplus \odot \odot \odot$

weeks (higher shade

group was 0.34

(3.12 higher to 3.88 higher)

indicates whiter)

\section{Adverse effects}

Not reported

${ }^{*}$ The risk in the intervention group (and its 95\% confidence interval) is based on the assumed risk in the comparison group and the relative effect of the intervention (and its $95 \% \mathrm{Cl}$

Cl: confidence interval; RCT: randomised controlled trial

\section{GRADE Working Group grades of evidence}

High certainty: we are very confident that the true effect lies close to that of the estimate of the effect

Moderate certainty: we are moderately confident in the effect estimate: the true effect is likely to be close to the estimate of the effect, but there is a possibility that it is substantially different

Low certainty: our confidence in the effect estimate is limited: the true effect may be substantially different from the estimate of the effect

Very low certainty: we have very little confidence in the effect estimate: the true effect is likely to be substantially different from the estimate of effect

1Downgraded for risk of bias - unclear risk of bias due to selection and detection bias.

2Downgraded for imprecision - single trial and low sample and event rate. 
Summary of findings 5. HP paint-on gel versus placebo for whitening teeth

Hydrogen peroxide (HP) paint-on gel compared to placebo for whitening teeth

Patient or population: adults undergoing bleaching

Setting: home-based

Intervention: HP paint-on gel

Comparison: placebo

\section{Tooth whitening - assessed by the dentist}

Comparisons

Anticipated absolute effects ${ }^{*}(95 \% \mathrm{Cl})$

Relative effect

Number of par-

ticipants

Certainty of the Comments

Risk with Risk with HP paint-on

(studies)

evidence

placebo

SMD was 0.67 higher in HP paint-on gel

(GRADE)

$6 \%$ HP paint-on gel versus

placebo - 2 weeks (higher

shade indicates whiter)

group

( 0.19 higher to 1.14 higher)

Adverse effects

Main adverse events reported in majority of trials were mild and transient tooth sensitivity and oral irritation which occurred more in the intervention group compared to placebo

${ }^{*}$ The risk in the intervention group (and its $95 \%$ confidence interval) is based on the assumed risk in the comparison group and the relative effect of the intervention (and its $95 \% \mathrm{Cl}$ )

CI: confidence interval; RCT: randomised controlled trial; SMD: standardised mean difference

\section{GRADE Working Group grades of evidence}

High certainty: we are very confident that the true effect lies close to that of the estimate of the effect

Moderate certainty: we are moderately confident in the effect estimate: the true effect is likely to be close to the estimate of the effect, but there is a possibility that it is substantially different

Low certainty: our confidence in the effect estimate is limited: the true effect may be substantially different from the estimate of the effect

Very low certainty: we have very little confidence in the effect estimate: the true effect is likely to be substantially different from the estimate of effect

1Downgraded for risk of bias - unclear risk of bias due to selection, performance, and detection bias.

2Downgraded for imprecision - low sample size and event rate. 


\begin{tabular}{|c|c|c|c|c|c|c|}
\hline \multicolumn{7}{|c|}{ Sodium hexametaphosphate (SHMP) chewing gum compared to placebo for whitening teeth } \\
\hline \multicolumn{7}{|c|}{$\begin{array}{l}\text { Patient or population: adults undergoing bleaching } \\
\text { Setting: home-based } \\
\text { Intervention: SHMP chewing gum } \\
\text { Comparison: placebo }\end{array}$} \\
\hline \multicolumn{7}{|c|}{ Tooth whitening - assessed by the dentist } \\
\hline \multirow[t]{2}{*}{ Comparisons } & \multicolumn{2}{|c|}{ Anticipated absolute effects ${ }^{\star}(95 \% \mathrm{Cl})$} & \multirow{2}{*}{$\begin{array}{l}\text { Relative effect } \\
(95 \% \mathrm{CI})\end{array}$} & \multirow{2}{*}{$\begin{array}{l}\text { Number of par- } \\
\text { ticipants } \\
\text { (studies) }\end{array}$} & \multirow{2}{*}{$\begin{array}{l}\text { Certainty of } \\
\text { the evidence } \\
\text { (GRADE) }\end{array}$} & \multirow[t]{2}{*}{ Comments } \\
\hline & Risk with placebo & Risk with SHMP chewing gum & & & & \\
\hline $\begin{array}{l}7.5 \% \text { SHMP chewing gum } \\
\text { versus placebo - } 2 \text { days } \\
\text { (higher shade indicates } \\
\text { whiter) }\end{array}$ & $\begin{array}{l}\text { The mean shade } \\
\text { change in placebo } \\
\text { gum was }-4.28\end{array}$ & $\begin{array}{l}\text { Mean difference in the shade change } \\
\text { was } 0.89 \text { higher in the SHMP chewing } \\
\text { gum group } \\
\text { ( } 0.77 \text { higher to } 1.01 \text { higher) }\end{array}$ & - & $\begin{array}{l}37 \\
(1 \mathrm{RCT})\end{array}$ & $\begin{array}{l}\oplus \odot \odot \odot \\
\text { VERY LOW } 1,2\end{array}$ & - \\
\hline $\begin{array}{l}5.6 \% \text { SHMP chewing gum } \\
\text { versus placebo - } 3 \text { days } \\
\text { (higher shade indicates } \\
\text { whiter) }\end{array}$ & $\begin{array}{l}\text { The mean shade } \\
\text { change in placebo } \\
\text { gum was }-7.27\end{array}$ & $\begin{array}{l}\text { Mean difference in the shade change } \\
\text { was } 2.60 \text { higher in the SHMP chewing } \\
\text { gum group } \\
\text { (1.45 higher to } 3.75 \text { higher) }\end{array}$ & - & $\begin{array}{l}20 \\
(1 \mathrm{RCT})\end{array}$ & $\begin{array}{l}\oplus \odot \odot \ominus \\
\text { VERY LOW } 1,2\end{array}$ & - \\
\hline $\begin{array}{l}4 \% \text { SHMP chewing gum } \\
\text { versus placebo - } 12 \text { weeks } \\
\text { (higher shade indicates } \\
\text { whiter) }\end{array}$ & $\begin{array}{l}\text { The mean shade } \\
\text { change in placebo } \\
\text { gum was }-1.27\end{array}$ & $\begin{array}{l}\text { Mean difference in the shade change } \\
\text { was } 0.14 \text { lower in the SHMP chewing } \\
\text { gum group } \\
\text { ( } 0.38 \text { lower to } 0.10 \text { higher) }\end{array}$ & - & $\begin{array}{l}108 \\
(1 \mathrm{RCT})\end{array}$ & $\begin{array}{l}\oplus \odot \odot \odot \\
\text { VERY LOW } 1,2\end{array}$ & - \\
\hline \multicolumn{7}{|l|}{ Adverse effects } \\
\hline \multicolumn{7}{|l|}{ Not reported } \\
\hline \multicolumn{7}{|c|}{$\begin{array}{l}\text { *The risk in the intervention group (and its } 95 \% \text { confidence interval) is based on the assumed risk in the comparison group and the relative effect of the intervention (an } \\
\text { its } 95 \% \mathrm{Cl} \text { ) }\end{array}$} \\
\hline \multicolumn{7}{|c|}{ Cl: confidence interval; $\mathbf{R C T}$ : randomised controlled trial } \\
\hline
\end{tabular}

\section{GRADE Working Group grades of evidence}

High certainty: we are very confident that the true effect lies close to that of the estimate of the effect

Moderate certainty: we are moderately confident in the effect estimate: the true effect is likely to be close to the estimate of the effect, but there is a possibility that it is substantially different

Low certainty: our confidence in the effect estimate is limited: the true effect may be substantially different from the estimate of the effect 
1Downgraded for risk of bias - unclear risk of bias due to selection, performance, and detection bias.

2Downgraded for imprecision - single trial and low sample and event rate.

Summary of findings 7 . STPP chewing gum versus placebo for whitening teeth

Sodium tripolyphosphate (STPP) chewing gum compared to placebo for whitening teeth

Patient or population: adults undergoing bleaching

Setting: home-based

Intervention: STPP chewing gum

Comparison: placebo

Tooth whitening - assessed by the dentist

Comparisons

Anticipated absolute effects ${ }^{*}(95 \% \mathrm{Cl})$

Relative effect

Number of par-

ticipants

Certainty of

Comments

Risk with placebo

Risk with STPP chewing gum

(studies)

(GRADE)

$1 \%$ STPP chewing gum The mean shade Mean difference in the shade change was

versus placebo - 6

108

0.18 higher in the STPP chewing gum

(1 RCT)

$\oplus \odot)^{2}$

weeks (higher shade gum was -0.09

(0.10 higher to 0.26 higher)

\section{Adverse effects}

Not reported

${ }^{\star}$ The risk in the intervention group (and its 95\% confidence interval) is based on the assumed risk in the comparison group and the relative effect of the intervention (and its $95 \% \mathrm{Cl}$ )

Cl: confidence interval; RCT: randomised controlled trial

\section{GRADE Working Group grades of evidence}

High certainty: we are very confident that the true effect lies close to that of the estimate of the effect

Moderate certainty: we are moderately confident in the effect estimate: the true effect is likely to be close to the estimate of the effect, but there is a possibility that it is

substantially different

Low certainty: our confidence in the effect estimate is limited: the true effect may be substantially different from the estimate of the effect

Very low certainty: we have very little confidence in the effect estimate: the true effect is likely to be substantially different from the estimate of effect 
1Downgraded for risk of bias - unclear risk of bias due to lack of allocation concealment.

2Downgraded for imprecision - single trial and low sample and event rate.

\section{Summary of findings 8 . HP mouthwash versus placebo for whitening teeth}

\section{Hydrogen peroxide (HP) mouthwash compared to placebo for whitening teeth}

Patient or population: adults undergoing bleaching

Setting: home-based

Intervention: HP mouthwash

Comparison: placebo

\section{Tooth whitening - assessed by the dentist}

Comparisons

Anticipated absolute effects ${ }^{\star}(95 \% \mathrm{Cl})$

Relative effect

Number of par-

Certainty of

Comments

Risk with Risk with HP

$(95 \% \mathrm{CI})$

ticipants

the evidence

placebo mouthwash

\section{$1.5 \% \mathrm{HP}+0.05 \% \mathrm{HF}$ mouth}

wash versus placebo - 6

months (higher OR indicates

Study population

whiter)

$\begin{array}{ll}0 \text { per } 1000 & 0 \text { per } 1000 \\ (0 \text { to } 0)\end{array}$

$\oplus \odot \odot \odot$

OR 10.89

(5.08 to 23.35$)$

$(1 \mathrm{RCT})$

Log of odds ratio and SE were calcu-

lated and data analyzed using gener-

ic inverse variance method

\section{Adverse effects}

Not reported

${ }^{\star}$ The risk in the intervention group (and its 95\% confidence interval) is based on the assumed risk in the comparison group and the relative effect of the intervention (and its $95 \% \mathrm{Cl}$ )

CI: confidence interval; F: fluoride; OR: odds ratio; $\mathbf{R C T}$ : randomised controlled trial; SE: standard error

\section{GRADE Working Group grades of evidence}

High certainty: we are very confident that the true effect lies close to that of the estimate of the effect

Moderate certainty: we are moderately confident in the effect estimate: the true effect is likely to be close to the estimate of the effect, but there is a possibility that it is substantially different

Low certainty: our confidence in the effect estimate is limited: the true effect may be substantially different from the estimate of the effect

Very low certainty: we have very little confidence in the effect estimate: the true effect is likely to be substantially different from the estimate of effect

1Downgraded for risk of bias - unclear risk of bias due to attrition bias.

2Downgraded for imprecision - low sample size and event rate. 


\section{Carbamide peroxide (CP) gel in tray compared to CP gel in tray for whitening teeth}

Patient or population: adults undergoing bleaching

Setting: home-based

Intervention: $\mathrm{CP}$ gel in tray

Comparison: $\mathrm{CP}$ gel in tray

\section{Tooth whitening - assessed by the dentist}

Comparisons

Anticipated absolute effects ${ }^{*}(95 \% \mathrm{Cl})$

Relative effect

Number of par-

Certainty of

Comments

Risk with CP gel in Risk with CP gel in tray

ticipants

the evidence

(studies) (GRADE)

tray

$10 \% \mathrm{CP}$ versus $10 \% \mathrm{CP}-2$

weeks (higher RR indicates

whiter)

Study population

66

RR 1.03

912 per 1000

937 per 1000

(704 to 990)

$10 \%$ CP versus $16 \% \mathrm{CP}$

2-year follow-up (higher

shade indicates whiter)

The mean after in-

Mean difference in shade change was

tervention in $10 \% \quad 1.20$ higher in $16 \%$ CP group

( 0.35 lower to 2.75 higher)

$16 \%$ CP versus $16 \% \mathrm{CP}+$

ACP - 6 months (higher

CP group was -81

The mean change

in $16 \%$ CP group

Mean difference in shade change was

0.78 higher in $16 \% \mathrm{CP}+\mathrm{ACP}$ group

(0.37 higher to 1.19 higher)

shade indicates whiter)

$5 \%$ CP versus $10 \%$ CP - 2

weeks (higher shade indi-

cates whiter)

The mean after

Mean difference in shade change was

intervention for $\quad 0.41$ higher in $5 \%$ CP group

$10 \%$ CP group was (2.17 lower to 2.98 higher)

(0.90 to 1.18$)$

(1 RCT)

VERY LOW 1,2

$-76.813$

$10 \%$ CP versus $15 \%$ CP - $2 \quad$ - $\quad$ Mean difference was 2.22 higher in $15 \%$

weeks (higher shade

cates whiter)

$\mathrm{CP}$ group

(1.29 higher to 3.15 higher)

$\begin{array}{ll}81 & \oplus \ominus \ominus \ominus \\ (1 \text { RCT }) & \text { VERY LOW } 1,2\end{array}$

$10 \% \mathrm{CP}$ versus $10 \% \mathrm{CP}+\mathrm{KN}$

Standardised mean difference was 0.32

higher in $10 \% \mathrm{CP}+\mathrm{KN}+\mathrm{NaF}$ group

27

$27 \quad \oplus \oplus \ominus \ominus$

$+\mathrm{NaF}-2$ weeks

( 0.20 lower to 0.84 higher)

$\begin{array}{ll}21 & \oplus \ominus \ominus \ominus \\ (1 \mathrm{RCT}) & \text { VERY LOW1, }\end{array}$

Tooth whitening - reported by the patient 
$10 \%$ CP versus $17 \% \mathrm{CP}-3$ weeks (higher shade indicates whiter)
The mean change in shade for $10 \%$

$\mathrm{CP}$ group was

Mean difference in patient contentment was 2.6 higher in $17 \% \mathrm{CP}$ group

(2.57 higher to 2.63 higher)

\section{Adverse effects}

Higher concentrations of CP in tray led to more tooth sensitivity and gingival irritation. However, the symptoms were mild and transient. CP in tray with desensitiser showed significantly less sensitivity compared to the groups without the desensitiser

${ }^{\star}$ The risk in the intervention group (and its $95 \%$ confidence interval) is based on the assumed risk in the comparison group and the relative effect of the intervention (and its $95 \% \mathrm{Cl}$ )

ACP: amorphous calcium phosphate; Cl: confidence interval; KN: potassium nitrate; NaF: sodium fluoride; RCT: randomised controlled trial; $\mathbf{R R}$ : risk ratio

\section{GRADE Working Group grades of evidence}

High certainty: we are very confident that the true effect lies close to that of the estimate of the effect

Moderate certainty: we are moderately confident in the effect estimate: the true effect is likely to be close to the estimate of the effect, but there is a possibility that it is substantially different

Low certainty: our confidence in the effect estimate is limited: the true effect may be substantially different from the estimate of the effect

Very low certainty: we have very little confidence in the effect estimate: the true effect is likely to be substantially different from the estimate of effect

1Downgraded for risk of bias - unclear risk of bias due to lack of allocation concealment.

2 Downgraded for imprecision - low sample size and event rate.

\section{Summary of findings 10. CP gel in tray versus HP gel in tray for whitening teeth}

\section{Carbamide peroxide (CP) gel in tray compared to hydrogen peroxide (HP) gel in tray for whitening teeth}

Patient or population: adults undergoing bleaching

Setting: home-based

Intervention: $\mathrm{CP}$ gel in tray

Comparison: HP gel in tray

\section{Tooth whitening - assessed by the dentist}

Comparisons
Anticipated absolute effects ${ }^{\star}(95 \% \mathrm{Cl})$

Risk with CP gel in

tray

\section{Relative effect}

$(95 \% \mathrm{Cl})$ ticipants

(studies)
Certainty of

the evidence

(GRADE)

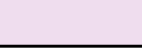

\section{Comments}

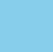




\begin{tabular}{|c|c|c|c|c|c|}
\hline $\begin{array}{l}10 \% \mathrm{CP} \text { versus } 7.5 \% \mathrm{HP}-2 \\
\text { weeks (higher shade indi- } \\
\text { cates whiter) }\end{array}$ & $\begin{array}{l}\text { The mean shade } \\
\text { change in the CP gel in } \\
\text { tray group was } 3.40\end{array}$ & $\begin{array}{l}\text { Mean difference in shade change was } \\
1 \text { lower in the HP group } \\
\text { ( } 2.86 \text { lower to } 0.86 \text { higher) }\end{array}$ & $\begin{array}{l}48 \\
(1 \mathrm{RCT})\end{array}$ & $\begin{array}{l}\oplus \ominus \ominus \ominus \\
\text { VERY LOW } 1,2\end{array}$ & - \\
\hline $\begin{array}{l}20 \% \text { CP versus } 9 \% \mathrm{HP} \text { - } 2 \\
\text { weeks (higher shade indi- } \\
\text { cates whiter) }\end{array}$ & $\begin{array}{l}\text { The mean shade } \\
\text { change in the CP gel in } \\
\text { tray group was }-6.97\end{array}$ & $\begin{array}{l}\text { Mean difference in shade change was } \\
0.58 \text { lower in the HP group } \\
\text { ( } 8.01 \text { lower to } 6.85 \text { higher) }\end{array}$ & $\begin{array}{l}37 \\
(1 \mathrm{RCT})\end{array}$ & $\begin{array}{l}\oplus \ominus \ominus \ominus \\
\text { VERY LOW } 1,2\end{array}$ & - \\
\hline $\begin{array}{l}20 \% \text { CP versus } 7.5 \% \mathrm{HP}- \\
12 \text { days (higher shade indi- } \\
\text { cates whiter) }\end{array}$ & $\begin{array}{l}\text { The mean shade } \\
\text { change in the CP gel in } \\
\text { tray group was }-2.59\end{array}$ & $\begin{array}{l}\text { Mean difference in shade change was } \\
0.99 \text { lower in the HP group } \\
\text { ( } 2.32 \text { lower to } 0.34 \text { higher) }\end{array}$ & $\begin{array}{l}56 \\
(1 \mathrm{RCT})\end{array}$ & $\begin{array}{l}\oplus \ominus \ominus \ominus \\
\text { VERY LOW } 1,2\end{array}$ & - \\
\hline $\begin{array}{l}20 \% \text { CP versus } 7.5 \% \mathrm{HP}-12 \\
\text { weeks (higher shade indi- } \\
\text { cates whiter) }\end{array}$ & $\begin{array}{l}\text { The mean shade } \\
\text { change in the CP gel in } \\
\text { tray group was }-2\end{array}$ & $\begin{array}{l}\text { Mean difference in shade change was } \\
0.25 \text { lower in the HP group } \\
\text { ( } 0.40 \text { lower to } 0.10 \text { lower) }\end{array}$ & $\begin{array}{l}24 \\
(1 \mathrm{RCT})\end{array}$ & $\begin{array}{l}\oplus \ominus \ominus \ominus \\
\text { VERY LOW } 1,2\end{array}$ & - \\
\hline $\begin{array}{l}10 \% \text { CP versus } 6 \% \text { HP (dark- } \\
\text { er shade) - } 2 \text { weeks (higher } \\
\text { shade indicates whiter) }\end{array}$ & $\begin{array}{l}\text { The mean shade } \\
\text { change in the CP gel in } \\
\text { tray group was }-11.10\end{array}$ & $\begin{array}{l}\text { Mean difference in shade change was } \\
4.30 \text { lower in the HP group } \\
\text { ( } 5.02 \text { lower to } 3.58 \text { lower) }\end{array}$ & $\begin{array}{l}164 \text { teeth } \\
\text { (1 RCT) }\end{array}$ & $\begin{array}{l}\oplus \ominus \ominus \ominus \\
\text { VERY LOW } 1,2\end{array}$ & $\begin{array}{l}\text { Analysis done } \\
\text { at tooth level }\end{array}$ \\
\hline $\begin{array}{l}10 \% \text { CP versus } 6 \% \text { HP } \\
\text { (medium dark and lighter } \\
\text { shade) - } 2 \text { weeks (higher } \\
\text { shade indicates whiter) }\end{array}$ & - & $\begin{array}{l}\text { Mean difference in shade change was } \\
2.22 \text { lower in the HP group } \\
\text { ( } 2.63 \text { lower to } 1.81 \text { lower) }\end{array}$ & $\begin{array}{l}349 \text { teeth } \\
(1 \mathrm{RCT})\end{array}$ & $\begin{array}{l}\oplus \ominus \ominus \ominus \\
\text { VERY LOW } 1,2\end{array}$ & $\begin{array}{l}\text { Analysis done } \\
\text { at tooth level }\end{array}$ \\
\hline Adverse effects & & & & & \\
\hline
\end{tabular}

${ }^{*}$ The risk in the intervention group (and its $95 \%$ confidence interval) is based on the assumed risk in the comparison group and the relative effect of the intervention (and its $95 \% \mathrm{Cl}$ )

Cl: confidence interval; $\mathbf{R C T}$ : randomised controlled trial

\section{GRADE Working Group grades of evidence}

High certainty: we are very confident that the true effect lies close to that of the estimate of the effect

Moderate certainty: we are moderately confident in the effect estimate: the true effect is likely to be close to the estimate of the effect, but there is a possibility that it is substantially different

Low certainty: our confidence in the effect estimate is limited: the true effect may be substantially different from the estimate of the effect

Very low certainty: we have very little confidence in the effect estimate: the true effect is likely to be substantially different from the estimate of effect

1Downgraded for risk of bias - unclear risk of bias due to lack of allocation concealment.

2Downgraded for imprecision - low sample size and event rate. 


\section{Hydrogen peroxide (HP) strips compared to carbamide peroxide (CP) gel in tray for whitening teeth}

Patient or population: adults undergoing bleaching

Setting: home-based

Intervention: HP strips

Comparison: $\mathrm{CP}$ gel in tray

\section{Tooth whitening - assessed by the dentist}

Comparisons

$6 \% \mathrm{HP}$ versus $5 \% \mathrm{CP}+5 \%$

$\mathrm{KN}$ - 1 week (higher shade

indicates whiter)

$6 \% \mathrm{HP}$ versus $10 \% \mathrm{CP}-2$

weeks (higher shade indi-

cates whiter)

$14 \%$ HP versus $35 \%$ CP -

30 days (higher shade indi-

cates whiter)

$6 \% \mathrm{HP}$ versus $10 \% \mathrm{CP}-6$

weeks (higher shade indi-

cates whiter)

$6.5 \% \mathrm{HP}$ versus $15 \% \mathrm{CP}-3$

months (higher shade indi-

cates whiter)

$6.5 \% \mathrm{HP}$ versus $16 \% \mathrm{CP}$ -

21 days (higher shade indi-

cates whiter)

\section{Anticipated absolute effects ${ }^{*}(95 \% \mathrm{CI})$}

Risk with CP gel in tray

The mean change in shade for the CP gel in tray was -1.20

-

$-$

The mean change in

shade for the CP gel in

tray was -4

The mean change in

shade for the CP gel in tray was -0.90

The mean change in shade for the CP gel in tray was -8.76

The mean change in shade for the CP gel in tray was 2.10

\section{Relative effect}

(95\% CI)

Risk with HP strips

Mean difference was 0.71 higher for

the strip group

1.35 lower to 0.07 lower)

Mean difference was 0.42 higher for

the strip group

(0.92 lower to 0.09 higher)

Mean difference was 0.58 lower for

the strip group

( 0.61 lower to 1.77 higher)

Mean difference was 0.30 higher for

the strip group

( 0.95 lower to 0.35 higher)

Mean difference was 3.15 lower for the strip group

( 0.15 lower to 6.45 lower)

Mean difference was 2.10 lower for

the strip group

Number of par- Certainty of

the evidence

Comments

ticipants

(GRADE)

32

$\oplus \ominus \odot \odot$

$149 \quad \oplus \odot \ominus \ominus$

(4 RCTs) VERY LOW1, 2

$24 \quad \oplus \ominus \ominus \ominus$

(1 RCT) VERY LOW 1,2

$36 \quad \oplus \ominus \ominus \ominus$

(1 RCT) VERY LOW1, 2

24

24

$\oplus \ominus \odot \odot$

(1.16 lower to 3.04 lower)

Adverse effects 


\begin{tabular}{|c|c|c|c|c|c|c|}
\hline \multicolumn{7}{|c|}{$\begin{array}{l}\text { When HP strips were compared to CP gel in tray, results were variable for adverse reactions (tooth sensitivity and oral irritation) with some trials favouring the strip group, } \\
\text { some favouring the tray group and some showing no differences between the groups }\end{array}$} \\
\hline \multicolumn{7}{|c|}{$\begin{array}{l}{ }^{*} \text { The risk in the intervention group (and its } 95 \% \text { confidence interval) is based on the assur } \\
\text { its } 95 \% \mathrm{CI} \text { ) } \\
\text { Cl: confidence interval; } \mathbf{K N} \text { : potassium nitrate; } \mathbf{R C T} \text { : randomised controlled trial }\end{array}$} \\
\hline \multicolumn{7}{|c|}{$\begin{array}{l}\text { GRADE Working Group grades of evidence } \\
\text { High certainty: we are very confident that the true effect lies close to that of the estimate of the effect } \\
\text { Moderate certainty: we are moderately confident in the effect estimate: the true effect is likely to be close to the estimate of the effect, but there is a possibility that it is } \\
\text { substantially different } \\
\text { Low certainty: our confidence in the effect estimate is limited: the true effect may be substantially different from the estimate of the effect } \\
\text { Very low certainty: we have very little confidence in the effect estimate: the true effect is likely to be substantially different from the estimate of effect }\end{array}$} \\
\hline \multicolumn{7}{|c|}{$\begin{array}{l}\text { 1Downgraded for risk of bias - unclear risk of bias due to lack of selection, performance and detection bias. } \\
\text { 2Downgraded for imprecision - low sample size and event rate. }\end{array}$} \\
\hline \multicolumn{7}{|c|}{ Hydrogen peroxide (HP) strips compared to HP gel in tray for whitening teeth } \\
\hline \multicolumn{7}{|c|}{$\begin{array}{l}\text { Patient or population: adults undergoing bleaching } \\
\text { Setting: home-based } \\
\text { Intervention: HP strips } \\
\text { Comparison: HP gel in tray }\end{array}$} \\
\hline \multicolumn{7}{|c|}{ Tooth whitening - assessed by the dentist } \\
\hline \multirow[t]{2}{*}{ Comparisons } & \multicolumn{2}{|c|}{ Anticipated absolute effects ${ }^{*}(95 \% \mathrm{Cl})$} & \multirow{2}{*}{$\begin{array}{l}\text { Relative effect } \\
(95 \% \mathrm{Cl})\end{array}$} & \multirow{2}{*}{$\begin{array}{l}\text { Number of par- } \\
\text { ticipants } \\
\text { (studies) }\end{array}$} & \multirow{2}{*}{$\begin{array}{l}\text { Certainty of } \\
\text { the evidence } \\
\text { (GRADE) }\end{array}$} & \multirow[t]{2}{*}{ Comments } \\
\hline & Risk with HP gel in tray & Risk with HP strips & & & & \\
\hline $\begin{array}{l}14 \% \text { HP strip versus } 9.5 \% \\
\text { HP gel in tray - } 22 \text { days } \\
\text { (higher shade indicates } \\
\text { whiter) }\end{array}$ & $\begin{array}{l}\text { The mean shade change } \\
\text { for HP gel in tray was } \\
-1.75\end{array}$ & $\begin{array}{l}\text { Mean difference was } 1.40 \text { higher for } \\
\text { the strip group } \\
\text { ( } 2.35 \text { lower to } 0.45 \text { lower) }\end{array}$ & - & $\begin{array}{l}29 \\
(1 \mathrm{RCT})\end{array}$ & $\begin{array}{l}\oplus \ominus \Theta \odot \\
\text { VERY LOW1, } 2\end{array}$ & - \\
\hline $\begin{array}{l}5.3 \% \text { HP strip versus } 5 \% \\
\text { HP gel in tray - } 18 \text { months } \\
\text { (higher shade indicates } \\
\text { whiter) }\end{array}$ & $\begin{array}{l}\text { The mean shade change } \\
\text { for HP gel in tray was - } \\
6.35\end{array}$ & $\begin{array}{l}\text { Mean difference was } 0.06 \text { higher for } \\
\text { the strip group } \\
\text { ( } 2.36 \text { lower to } 2.24 \text { higher) }\end{array}$ & - & $\begin{array}{l}28 \\
(1 \mathrm{RCT})\end{array}$ & $\begin{array}{l}\oplus \odot \Theta \odot \\
\text { VERY LOW1, } 2\end{array}$ & - \\
\hline
\end{tabular}

igh certainty: we are very confident that the tru

substantially different

Low certainty: our confidence in the effect estimate is limited: the true effect may be substantially different from the estimate of the effect

Very low certainty: we have very little confidence in the effect estimate: the true effect is likely to be substantially different from the estimate of effect

1Downgraded for risk of bias - unclear risk of bias due to lack of selection, performance and detection bias.

2Downgraded for imprecision - low sample size and event rate.

Summary of findings 12 . HP strips versus HP gel in tray for whitening teeth

Hydrogen peroxide (HP) strips compared to HP gel in tray for whitening teeth

Comparison: HP gel in tray

Tooth whitening - assessed by the dentist 


\begin{tabular}{|c|c|c|c|c|c|}
\hline \multicolumn{6}{|l|}{ Patient comfort } \\
\hline $\begin{array}{l}5.3 \% \text { HP strip versus } 5 \% \\
\text { HP gel in tray }\end{array}$ & $\begin{array}{l}\text { The mean patient accep- } \\
\text { tance for HP gel in tray } \\
\text { was } 2.23\end{array}$ & $\begin{array}{l}\text { Mean difference in patient accep- } \\
\text { tance was } 1.27 \text { lower for the strip } \\
\text { group } \\
\text { ( } 0.13 \text { higher to } 2.41 \text { higher) }\end{array}$ & - & $\begin{array}{l}28 \\
(1 \mathrm{RCT})\end{array}$ & $\begin{array}{l}\oplus \odot \Theta \odot \\
\text { VERY LOW1, } 2\end{array}$ \\
\hline \multicolumn{6}{|l|}{ Adverse effects } \\
\hline \multicolumn{6}{|c|}{ Tooth sensitivity and oral irritation were mild and transient and did not differ between the groups } \\
\hline \multicolumn{6}{|c|}{$\begin{array}{l}{ }^{\star} \text { The risk in the intervention group (and its } 95 \% \text { confidence interval) is based on the assumed risk in the comparison group and the relative effect of the intervention (and } \\
\text { its } 95 \% \mathrm{Cl} \text { ) }\end{array}$} \\
\hline \multicolumn{6}{|c|}{$\begin{array}{l}\text { GRADE Working Group grades of evidence } \\
\text { High certainty: we are very confident that the true effect lies close to that of the estimate of the effect } \\
\text { Moderate certainty: we are moderately confident in the effect estimate: the true effect is likely to be close to the estimate of the effect, but there is a possibility that it is } \\
\text { substantially different } \\
\text { Low certainty: our confidence in the effect estimate is limited: the true effect may be substantially different from the estimate of the effect } \\
\text { Very low certainty: we have very little confidence in the effect estimate: the true effect is likely to be substantially different from the estimate of effect }\end{array}$} \\
\hline \multicolumn{6}{|c|}{$\begin{array}{l}\text { 1Downgraded for risk of bias - unclear risk of bias due to lack of selection, performance and detection bias. } \\
\text { 2Downgraded for imprecision - single trials, low sample and event rate. }\end{array}$} \\
\hline \multicolumn{6}{|c|}{ Summary of findings 13. HP strips versus HP strips (different concentrations) for whitening teeth } \\
\hline \multicolumn{6}{|c|}{ Hydrogen peroxide (HP) strips compared to HP strips for whitening teeth } \\
\hline \multicolumn{6}{|c|}{$\begin{array}{l}\text { Patient or population: adults undergoing bleaching } \\
\text { Setting: home-based } \\
\text { Intervention: HP strips } \\
\text { Comparison: HP strips }\end{array}$} \\
\hline \multicolumn{6}{|c|}{ Tooth whitening - assessed by the dentist } \\
\hline \multirow[t]{2}{*}{ Comparisons } & \multicolumn{2}{|c|}{ Anticipated absolute effects ${ }^{\star}(95 \% \mathrm{Cl})$} & \multirow{2}{*}{$\begin{array}{l}\text { Relative effect } \\
(95 \% \mathrm{Cl})\end{array}$} & \multirow{2}{*}{$\begin{array}{l}\text { Number of par- } \\
\text { ticipants } \\
\text { (studies) }\end{array}$} & \multirow{2}{*}{$\begin{array}{l}\text { Certainty of } \\
\text { the evidence } \\
\text { (GRADE) }\end{array}$} \\
\hline & Risk with $10 \%$ HP strips & Risk with HP strips & & & \\
\hline
\end{tabular}


$6 \% \mathrm{HP}$ versus $10 \% \mathrm{HP}$ thin gel - 15 days (higher shade indicates whiter)

9.5\% adhesion HP strips versus 10\% HP strips - day 9 (higher shade indicates whiter)
The mean shade change in $10 \%$ thin HP gel strip group was -3.03

The mean shade change $-2.30$
Mean difference in $6 \%$ HP strip

group was 0.68 lower

(0.16 higher to 1.20 higher)
Mean difference in $9.5 \%$ adhesion

strip group was 1.50 higher

29

(1 RCT)

VERY LOW 1,2

\section{Adverse effects}

When HP strips were compared to HP strips, very thin gel had lesser tooth sensitivity compared to thicker gel even though the concentration of HP was higher. Strips applied for 2 hours had greater symptoms of sensitivity compared with 30-minute group. However, these results were not significant

${ }^{*}$ The risk in the intervention group (and its $95 \%$ confidence interval) is based on the assumed risk in the comparison group and the relative effect of the intervention (and its $95 \% \mathrm{Cl}$ )

Cl: confidence interval; $\mathbf{R C T}$ : randomised controlled trial

\section{GRADE Working Group grades of evidence}

High certainty: we are very confident that the true effect lies close to that of the estimate of the effect

Moderate certainty: we are moderately confident in the effect estimate: the true effect is likely to be close to the estimate of the effect, but there is a possibility that it is

substantially different

Low certainty: our confidence in the effect estimate is limited: the true effect may be substantially different from the estimate of the effect

Very low certainty: we have very little confidence in the effect estimate: the true effect is likely to be substantially different from the estimate of effect

1Downgraded for risk of bias - unclear risk of bias due to lack of selection, performance, detection and attrition bias.

2Downgraded for imprecision - single trials, low sample and event rate.

\section{Summary of findings 14. CP paint-on gel versus HP strips for whitening teeth}

Carbamide peroxide (CP) paint-on gel compared to hydrogen peroxide (HP) strips for whitening teeth

Patient or population: adults undergoing bleaching

Setting: home-based

Intervention: $\mathrm{CP}$ paint-on

Comparison: HP strips

Tooth whitening - assessed by the dentist

Comparison Anticipated absolute effects ${ }^{*}(95 \% \mathrm{CI})$

Relative effect

$(95 \% \mathrm{Cl})$
Number of par-

ticipants

(studies)
Certainty of the

Comments

evidence

(GRADE) 


\begin{tabular}{|c|c|c|c|c|c|c|}
\hline & $\begin{array}{l}\text { Risk with CP } \\
\text { paint-on }\end{array}$ & \multicolumn{3}{|l|}{ Risk with HP strips } & & \\
\hline $\begin{array}{l}18 \% \text { CP paint-on gel ver- } \\
\text { sus } 6 \% \text { HP strips (higher } \\
\text { shade indicates whiter) }\end{array}$ & - & $\begin{array}{l}\text { Standardised mean difference for HP strip } \\
\text { group was } 1.50 \text { higher } \\
\text { (1.06 higher to } 1.94 \text { higher) }\end{array}$ & - & $\begin{array}{l}102 \\
(2 \mathrm{RCTs})\end{array}$ & \multicolumn{2}{|l|}{$\begin{array}{l}\oplus \odot \odot \odot \\
\text { VERY LOW1, } 2\end{array}$} \\
\hline \multicolumn{7}{|l|}{ Adverse effects } \\
\hline \multicolumn{7}{|l|}{ Not reported } \\
\hline \multicolumn{7}{|c|}{$\begin{array}{l}{ }^{*} \text { The risk in the intervention group (and its } 95 \% \text { confidence interval) is based on the assumed risk in the comparison group and the relative effect of the intervention (and } \\
\text { its } 95 \% \mathrm{Cl} \text { ) }\end{array}$} \\
\hline \multicolumn{7}{|c|}{$\begin{array}{l}\text { GRADE Working Group grades of evidence } \\
\text { High certainty: We are very confident that the true effect lies close to that of the estimate of the effect } \\
\text { Moderate certainty: We are moderately confident in the effect estimate: The true effect is likely to be close to the estimate of the effect, but there is a possibility that it is } \\
\text { substantially different } \\
\text { Low certainty: Our confidence in the effect estimate is limited: The true effect may be substantially different from the estimate of the effect } \\
\text { Very low certainty: We have very little confidence in the effect estimate: The true effect is likely to be substantially different from the estimate of effect }\end{array}$} \\
\hline \multicolumn{7}{|c|}{$\begin{array}{l}\text { 1Downgraded for risk of bias - unclear risk of bias due to lack of selection, performance and detection bias. } \\
\text { 2Downgraded for imprecision - low sample size and event rate. }\end{array}$} \\
\hline \multicolumn{7}{|c|}{ Summary of findings 15 . HP paint-on gel versus HP strips for whitening teeth } \\
\hline \multicolumn{7}{|c|}{ Hydrogen peroxide (HP) paint-on gel compared to HP strips for whitening teeth } \\
\hline \multicolumn{7}{|c|}{$\begin{array}{l}\text { Patient or population: adults undergoing bleaching } \\
\text { Setting: home-based } \\
\text { Intervention: HP paint-on } \\
\text { Comparison: HP strips }\end{array}$} \\
\hline \multicolumn{7}{|c|}{ Tooth whitening - assessed by the dentist } \\
\hline \multirow[t]{2}{*}{ Comparisons } & \multicolumn{2}{|c|}{ Anticipated absolute effects ${ }^{\star}(95 \% \mathrm{Cl})$} & Relative effect & \multirow{2}{*}{$\begin{array}{l}\text { Number of par- } \\
\text { ticipants } \\
\text { (studies) }\end{array}$} & \multirow{2}{*}{\multicolumn{2}{|c|}{$\begin{array}{l}\text { Certainty of } \\
\text { the evidence } \\
\text { (GRADE) }\end{array}$}} \\
\hline & $\begin{array}{l}\text { Risk with } 5.9 \% \text { HP } \\
\text { paint-on }\end{array}$ & Risk with $5.9 \%$ HP strip & & & & \\
\hline
\end{tabular}

$\bullet$ 


\begin{tabular}{|c|c|c|c|c|c|}
\hline \multicolumn{2}{|c|}{ 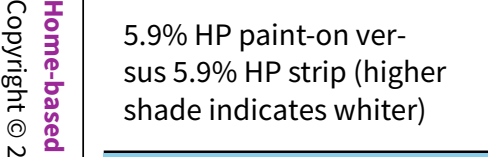 } & - & $\begin{array}{l}\text { Mean difference in } 5.90 \% \text { HP strip group } \\
\text { was } 2.70 \text { higher } \\
\text { ( } 2.08 \text { higher to } 3.32 \text { higher) }\end{array}$ & $\begin{array}{l}40 \\
(1 \mathrm{RCT})\end{array}$ & $\begin{array}{l}\oplus \odot \odot \ominus \\
\text { VERY LOW1, } 2\end{array}$ \\
\hline & \multicolumn{5}{|c|}{ Tooth whitening - reported by the patient } \\
\hline 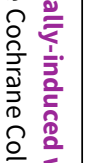 & $\begin{array}{l}5.9 \% \text { HP paint-on ver- } \\
\text { sus } 5.9 \% \text { HP strip (higher } \\
\text { shade indicates whiter) }\end{array}$ & $\begin{array}{l}\text { The mean patient } \\
\text { satisfaction for } \\
5.90 \% \text { HP paint on } \\
\text { gel group was }-4\end{array}$ & $\begin{array}{l}\text { Mean difference in patient satisfaction } \\
\text { was } 0.25 \text { lower for } 5.90 \% \text { HP strip group } \\
\text { (1.88 lower to } 1.38 \text { higher) }\end{array}$ & $\begin{array}{l}40 \\
(1 \mathrm{RCT})\end{array}$ & $\begin{array}{l}\oplus \odot \odot \ominus \\
\text { VERY LOW1, } 2\end{array}$ \\
\hline 高突 & \multicolumn{5}{|l|}{ Adverse effects } \\
\hline 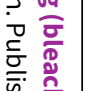 & \multicolumn{5}{|c|}{$\begin{array}{l}\text { Adverse events were mild in severity, and did not contribute to any treatment modification or early withdrawal. Slightly higher tooth hypersensitivity and gingival irritation } \\
\text { in the strip group was found although there was no evidence of a difference between the groups }\end{array}$} \\
\hline 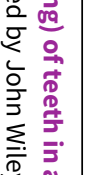 & \multicolumn{5}{|c|}{$\begin{array}{l}{ }^{*} \text { The risk in the intervention group (and its } 95 \% \text { confidence interval) is based on the assumed risk in the comparison group and the relative effect of the intervention (anc } \\
\text { its } 95 \% \mathrm{Cl} \text { ) }\end{array}$} \\
\hline 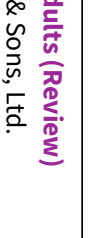 & \multicolumn{5}{|c|}{$\begin{array}{l}\text { GRADE Working Group grades of evidence } \\
\text { High certainty: we are very confident that the true effect lies close to that of the estimate of the effect } \\
\text { Moderate certainty: we are moderately confident in the effect estimate: the true effect is likely to be close to the estimate of the effect, but there is a possibility that it is } \\
\text { substantially different } \\
\text { Low certainty: our confidence in the effect estimate is limited: the true effect may be substantially different from the estimate of the effect } \\
\text { Very low certainty: we have very little confidence in the effect estimate: the true effect is likely to be substantially different from the estimate of effect }\end{array}$} \\
\hline & \multicolumn{5}{|c|}{$\begin{array}{l}\text { 1Downgraded for risk of bias - unclear risk of bias due to lack of selection, performance and detection bias. } \\
\text { 2Downgraded for imprecision - single trial, low sample and event rate. }\end{array}$} \\
\hline & \multicolumn{5}{|c|}{ Summary of findings 16. CP paint-on versus CP paint-on (different concentrations) for whitening teeth } \\
\hline & \multicolumn{5}{|c|}{ Carbamide peroxide (CP) paint-on compared to CP paint-on for whitening teeth } \\
\hline & \multicolumn{5}{|c|}{$\begin{array}{l}\text { Patient or population: adults undergoing bleaching } \\
\text { Setting: home-based } \\
\text { Intervention: CP paint-on } \\
\text { Comparison: CP paint-on }\end{array}$} \\
\hline & \multicolumn{5}{|c|}{ Tooth whitening - assessed by the dentist } \\
\hline
\end{tabular}


Comparison: HP paint-on

Tooth whitening - assessed by the dentist

Comparison

Anticipated absolute effects* $(95 \% \mathrm{Cl})$

Relative effect

Relative
$(95 \% \mathrm{Cl})$

Number of par-

ticipants

Certainty of

the evidence

Comments

Risk with CP paint-on Risk with HP paint-on

$25 \%$ CP paint-on ver-

sus $8.75 \%$ HP paint-

The mean change in shade Mean difference in shade change was

(studies)

(GRADE)

on (higher shade indi-

in $25 \%$ CP paint-on

0.16 lower in $8.75 \% \mathrm{HP}$ paint-on group

59

was 6.54

(1.39 lower to 1.07 higher)

$\oplus \ominus \ominus \odot$

(1 RCT)

VERY LOW 1,2

cates whiter)

\section{Adverse effects}

Not reported

${ }^{*}$ The risk in the intervention group (and its 95\% confidence interval) is based on the assumed risk in the comparison group and the relative effect of the intervention (and its $95 \% \mathrm{Cl}$ )

Cl: confidence interval; $\mathbf{R C T}$ : randomised controlled trial

\section{GRADE Working Group grades of evidence}

High certainty: we are very confident that the true effect lies close to that of the estimate of the effect

Moderate certainty: we are moderately confident in the effect estimate: the true effect is likely to be close to the estimate of the effect, but there is a possibility that it is substantially different

Low certainty: our confidence in the effect estimate is limited: the true effect may be substantially different from the estimate of the effect

Very low certainty: we have very little confidence in the effect estimate: the true effect is likely to be substantially different from the estimate of effect

1Downgraded for risk of bias - unclear risk of bias due to lack of selection, performance and detection bias.

2Downgraded for imprecision - single trial, low sample and event rate.

\section{Summary of findings 18. HP paint-on versus HP paint-on for whitening teeth}

Hydrogen peroxide (HP) paint-on compared to HP paint-on for whitening teeth

Patient or population: adults undergoing bleaching

Setting: home-based

Intervention: HP paint-on

Comparison: HP paint-on

Tooth whitening - assessed by the dentist

N 


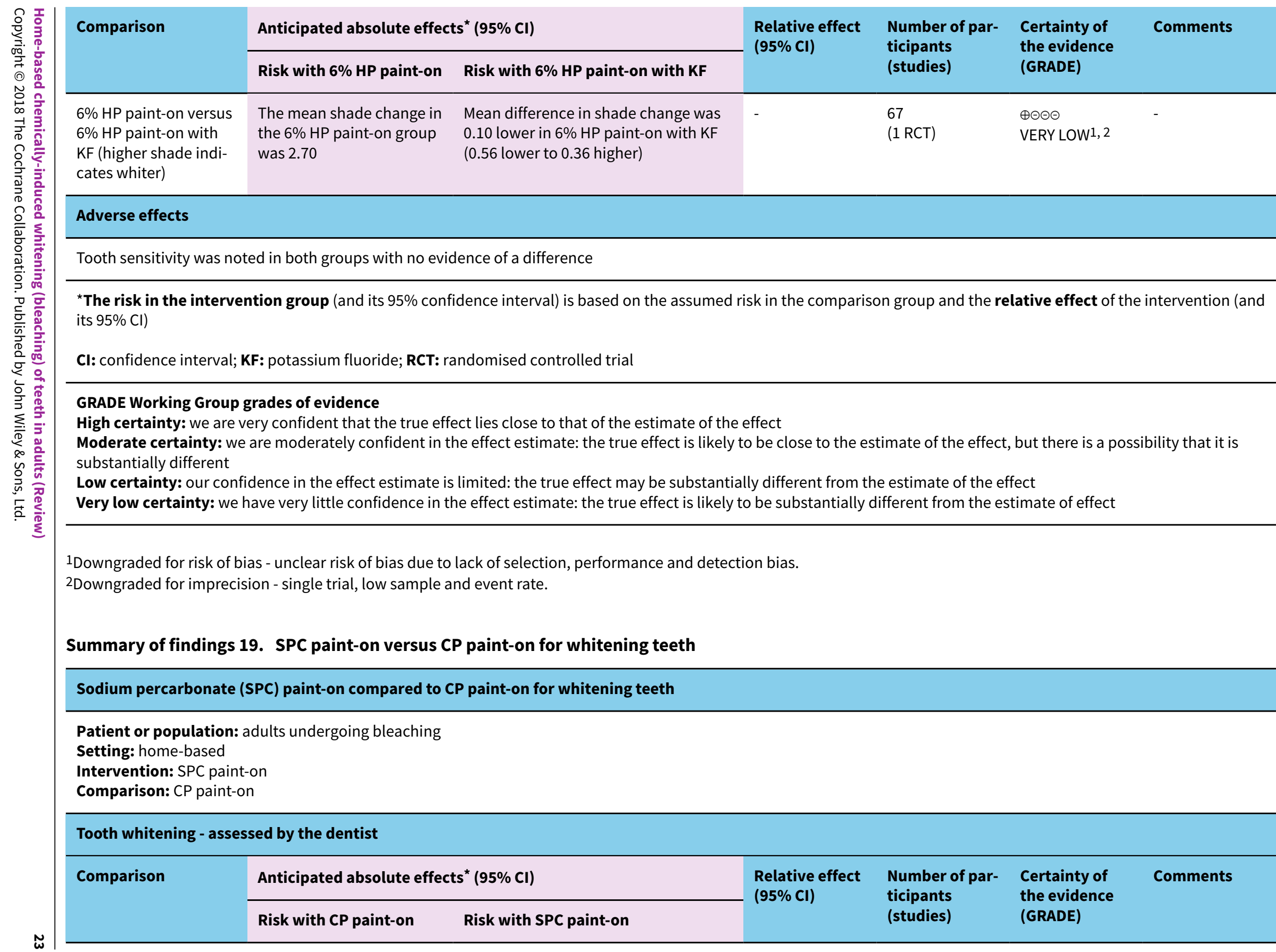


1 subject in 19\% SPC paint-on group reported oral sensitivity. All adverse events were symptomatic and mild in severity

* The risk in the intervention group (and its $95 \%$ confidence interval) is based on the assumed risk in the comparison group and the relative effect of the intervention (and its $95 \% \mathrm{Cl}$ )

Cl: confidence interval; $\mathbf{R C T}$ : randomised controlled trial

\section{GRADE Working Group grades of evidence}

High certainty: we are very confident that the true effect lies close to that of the estimate of the effect

Moderate certainty: we are moderately confident in the effect estimate: the true effect is likely to be close to the estimate of the effect, but there is a possibility that it is substantially different

Low certainty: our confidence in the effect estimate is limited: the true effect may be substantially different from the estimate of the effect

Very low certainty: we have very little confidence in the effect estimate: the true effect is likely to be substantially different from the estimate of effect

1Downgraded for risk of bias - unclear risk of bias due to lack of selection and detection bias.

2Downgraded for imprecision - single trial, low sample and event rate. 


\section{B A C K G R O U N D}

Aesthetic dentistry has received increased attention in recent years, especially because people are more concerned about the aesthetic appearance of their smile (Demarco 2009). Technological innovations in dentistry have been added due to patients' desire to improve aesthetics of their teeth, which is an important aspect of quality of life (Pinto 2014). A survey conducted in the UK revealed that $28 \%$ of adults were dissatisfied with their smile and among 3215 subjects examined, 50\% had some kind of tooth discolouration (Joiner 2006). Another survey performed in the UK in 2004 suggested that the public is concerned about dental appearance in terms of tooth colours (Alkhatib 2004). Based on the survey conducted by the American Academy of Cosmetic Dentistry in 2012, discoloured, stained or yellow teeth were the main reason for an unattractive smile. The same survey reported that there was a $29 \%$ increase in patients receiving tooth whitening in a span of 1 year and was expected to increase to $45 \%$ and more in the years to come. $70 \%$ of patients who opted for bleaching were females. $75 \%$ of respondents reported the use of at-home or over-the-counter whitening products. $18 \%$ of dentists in the US recommended a home-based bleaching method (Whitening survey 2012).

With this increased demand for whiter teeth, tooth whitening products have been exponentially increasing in the past few decades. Presently, tooth whitening products are the most popularly marketed oral care products (Whitening survey 2012).

\section{Description of the condition}

Tooth discolouration can be described as any change in the colour or translucency of a tooth and can be classified based on aetiology as extrinsic or intrinsic discolourations. Extrinsic discolourations adhere to the tooth surface (superficial stains), while intrinsic discolourations are integrated in the structure of teeth (Demarco 2009). However, in some cases, both intrinsic and extrinsic discolourations may affect tooth enamel, dentine or pulp.

\section{Extrinsic staining}

This usually results from accumulation of chromatogenic substances on the external tooth surface. These include smoking, pigments in foods and beverages, and metals such as iron or copper which lead to dark, brownish discolourations. These stains are localised mainly in the pellicle and are either generated by the reaction between sugars and amino acids or acquired from the retention of exogenous chromophores in the pellicle (Viscio 2000). This reaction is called Maillard reaction or the nonenzymatic browning reaction. Most extrinsic stains can be removed by routine prophylactic procedures. With time, these stains darken and become more persistent but they are highly responsive to bleaching.

\section{Intrinsic staining}

This can result from genetic disorders such as dentinogenesis imperfecta, amelogenesis imperfecta, thalassaemia, sickle cell anaemia, antibiotics such as tetracyclines, high levels of fluoride intake, dental caries, pulpal haemorrhage, pulpal necrosis, pulp tissue remnants, root filling materials/endodontic irrigation, or amalgam restorations (Nathoo 1997; Kim 2010; Belobrov 2011; Carey 2014; Kolosowski 2014). Likewise, high fevers during the time of a tooth development may cause enamel hypoplasia that leads to banding-type discolourations on the affected tooth surface. Aging is another common cause of discolouration. Over time, dentine tends to darken due to the formation of secondary dentine and the overlying enamel becomes thinner. Intrinsic stains cannot be removed by regular prophylactic procedures. However, they can be reduced by bleaching with agents penetrating the enamel and dentine to oxidize the chromogens, in some conditions.

\section{Description of the intervention}

Tooth whitening or bleaching is a procedure most commonly employed by professionals and patients. It is considered the least invasive aesthetic treatment for improving the appearance of discoloured teeth (Pinto 2014). It may be accomplished by physical removal of the stain or a chemical reaction (bleaching) to lighten the tooth colour. The active ingredient in most chemically-induced whitening products is hydrogen peroxide $\left(\mathrm{H}_{2} \mathrm{O}_{2}\right)$ which is delivered as hydrogen peroxide (HP) or carbamide peroxide (CP). CP is a stable complex, which will break down to HP and urea, once in contact with water. The basic bleaching action is due to the HP, which can be explained in three phases (Joshi 2016):

- initial phase: diffusion of HP through the inter-prismatic spaces and circulation within the tooth for 2 weeks;

- second phase: interaction of HP with organic chromophores which can be influenced by temperature, $\mathrm{pH}$, light and metal cations (Kwon 2015);

- third phase: colour change through an altered tooth surface.

Bleaching action reaches an end point which is known as inherent lightness potential for that tooth (Matis 2000). Usually if there is no improvement in the shade after 6 weeks of bleaching, irrespective of the bleaching agent and concentration, then bleaching is assumed to have reached its end point (Joshi 2016).

\section{Types of dental bleaching procedures}

Tooth discolourations can be improved by several methods such as internal bleaching for non-vital teeth, or external bleaching for vital teeth (Joiner 2006) or a combination of techniques.

\section{Internal bleaching/non-vital bleaching}

It consists of walking bleach and thermocatalytic bleaching techniques and is done after endodontic treatment by the dentist and comprises of in-office techniques, which are not in the scope of this review.

\section{External bleaching methods/vital bleaching}

There are three fundamental approaches for bleaching vital teeth.

1. In-office or power bleaching (not in the scope of this review).

2. At-home or dentist-supervised bleaching.

3. Consumer-purchased or over-the-counter (OTC) products which are available in pharmacies or supermarkets without any prescription or professional monitoring.

(Other non-dental products like malic acid found in juice of green apples or do-it-yourself whitening with strawberry and baking soda were reported (Kwon 2015) but are not in the scope of this review.)

This review includes only at-home or dentist-supervised bleaching and consumer-purchased or OTC products. 


\section{Home-based bleaching methods (dentist-supervised and OTC)}

A variety of peroxide compounds, including hydrogen peroxide (HP), carbamide peroxide (CP) or urea peroxide, sodium percarbonate (SPC), sodium hexametaphosphate (SHMP), sodium tripolyphosphate (STPP), and calcium peroxide have been used as active ingredients in home-based bleaching methods. These agents are supplied in different concentrations, used with varying application times and duration of treatment (Alqahtani 2014), and delivered in various forms.

\section{Gels in trays}

Dentist-supervised home-use tooth bleaching with custom trays is the most common bleaching procedure dispensed by dentists to their patients. Usually, this treatment modality consists of fabrication of a custom tray with and without reservoirs (Javaheri 2000; Caballero 2006; Baroudi 2014).

\section{Whitening strips}

These strips mainly contain hydrogen peroxide as the active agent in different concentrations. They are applied directly to the tooth surfaces and are thin flexible polyethylene strips coated with the bleaching gel. Continued research led to the development of stripbased whitening with very thin peroxide gels less than $0.20 \mathrm{~mm}$ in thickness (Perdigão 2004; Duschner 2006).

Disadvantages of the strip system are that it can reach only a finite number of teeth, cannot adapt well on malposed teeth, may interfere with speech patterns and can impinge on gingiva.

\section{Paint-on gels}

Paint-on gels or varnishes are barrier-free whitening products that present hydrogen or carbamide peroxide in a suspension that is brushed by an applicator over the tooth surface and which adheres to enamel. Some paint-on gels have sodium percarbonate as their active ingredient. This method has gained popularity since the consumer just needs to paint a thin layer of whitening gel on their teeth (similar to nail polish application on finger nails). The added advantage is that the users can scallop the product around the gingiva and apply it to an unlimited number of teeth, regardless of the position in the arch.

A disadvantage of this method is that lesser contact time of these agents to the tooth surface may result in reduced whitening of teeth. In addition, the applicator brush is re-used and stored in the gel product which might lead to microbial contamination. Hence, some manufacturers supply disposable cotton bud applicators for this purpose (Goldstein 1995; Carey 2014).

\section{Whitening mouthrinses}

Whitening mouthrinses prevent stains and fight plaque buildup. Generally, a low concentration of hydrogen peroxide $(1.5 \%)$, sodium hexametaphosphate have been included in the formulation to protect the teeth surface from new stains (Lima 2012).

\section{Whitening chewing gums}

These are well accepted and enjoyed by many as a frequent activity among children and adults, therefore, may be a means for local drug administration into the oral cavity (Barabolak 1991). Chewing gum based products possess a number of therapeutic benefits, including increased saliva flow and removal of food debris, plaque and surface stains (Walters 2004). Baking soda (Mankodi 2001; Soparkar 2001), sodium hexametaphosphate (White 2002; Walters 2004), and sodium tripolyphosphate (Shellis 2005; Porciani 2010) have been reported as the active ingredients in chewing gums.

\section{Whitening dentifrices}

The active components of tooth whitening dentifrices include hydrogen peroxide or carbamide peroxide which break down the organic molecules of biological film (Horn 2014). Additionally, abrasives such as alumina, dicalcium phosphate dihydrate and silica are also present in the formulation to promote stain removal (Demarco 2009). Their stain removal ability is related to the large quantity of abrasives in their formulation, which remove superficial extrinsic stains. However, the toothpaste abrasiveness needs to be moderated in order to prevent excessive wear to the underlying enamel and dentine. Toothpastes containing blue covarine, a pigment, which increases the perception of tooth whiteness are available in the market as bleaching toothpastes (Dantas 2015). Whitening dentifrices with desensitizing agents (Po 2014) to reduce the adverse event of sensitivity or dentifrices with casein phosphopeptide-amorphous calcium phosphate (CPP-ACP) to remineralize the enamel have been in use (de Vasconcelos 2012).

\section{Whitening dental floss}

Whitening dental floss has been introduced to promote stain reduction around the interproximal and subgingival areas. The stain removal properties are associated with the presence of silica in the composition, which promotes a superficial surface abrasion during application in the interdental region (Demarco 2009).

Whitening dentifrices, floss and toothbrushes which involve an abrasive action are not included in this review.

All the above products are either prescribed by the dentist for use at home or purchased by the consumer over-the-counter without professional consultation. The permitted concentration of $\mathrm{HP}$ varies between countries.

The at-home technique offers many advantages:

- self-administration by the patient

- less chair-side time

- high degree of safety

- fewer adverse effects

- low cost.

However, there are certain disadvantages:

- results dependent on active patient compliance and diligence of use

- high dropout rates (Leonard 2003)

- excessive use by overzealous patients leads to thermal sensitivity, reported to be as high as $67 \%$ (Haywood 1992).

\section{How the intervention might work}

\section{General mechanism of action}

\section{Hydrogen peroxide and carbamide peroxide formulations used as gels in trays/strips/paint-on gels and mouthrinses}

Tooth stains consist of compounds that have colour or darker shades called chromogens. Bleaching mainly constitutes removal 
of stains by chemical degradation of these chromogens. It is hypothesized that the basic chemistry of peroxide-based whitening agents is attributed to reaction of hydrogen peroxide with the chromogens. Carbamide peroxide is also an active ingredient in many whitening products. It is a stable complex which breaks down in contact with water to release hydrogen peroxide $(10 \% \mathrm{CP}$ on contact with water, gets converted to $3 \% \mathrm{HP}$ and $7 \%$ urea).

Chromogens fall into two categories: large organic compounds that have conjugated double bonds in their chemical structure and metal containing compounds. Bleaching of the organic compounds by hydrogen peroxide involves reacting with the double bonds to oxidize the double bond.This causes the chromogen to become a lighter coloured compound. Other hypothesized mechanisms include oxidation of proteins within the tooth matrix.

Bleaching of the metallic compounds is much more difficult. There are some professional products that contain sodium hypochlorite $(\mathrm{NaOCl})$ which react with the double bonds of the chromogen in much the same way as peroxide (Carey 2014).

\section{Sodium percarbonate used in paint-on gel}

$19 \%$ sodium percarbonate has been developed to deliver peroxide over a sustained period without a fixed barrier. The anhydrous system has peroxide bound in a silicone polymer suspension. Applied with a brush to the dried tooth surface, the suspension is designed to form an enamel adherent substantive film that hydrates overnight to slowly release peroxide into the tooth.

\section{Sodium hexametaphosphate used in chewing gums}

This active ingredient is a high molecular weight condensed phosphate analogue which inhibits stain chromogen adsorption reducing overall extrinsic staining.

\section{Sodium tripolyphosphate (STPP) used in chewing gums}

The inhibitory action of hydroxy apatite bound STPP on adsorption of salivary proteins, makes it an effective agent for inhibiting and removing dental stain.

\section{Why it is important to do this review}

The first published version of this Cochrane Review concluded that there was evidence that whitening products work when compared with placebo/no treatment (Hasson 2006). However, all trials in that review were short term and the majority of the included studies had low methodological quality (i.e. high risk of bias). Moreover, in the past 12 years there may have been additional randomised controlled trials published, which needed to be considered in the review. Therefore, there was a need to update this review to identify new evidence from pragmatic long-term clinical trials and also to re-look at the outcomes and comparisons used in the previous version.

\section{O B JE C T IVES}

To evaluate the effects of home-based tooth whitening products with chemical bleaching action, dispensed by a dentist or over-thecounter.

\section{METHODS}

\section{Criteria for considering studies for this review}

\section{Types of studies}

\section{Inclusion criteria}

- Randomised controlled clinical trials comparing dentistdispensed or over-the-counter (OTC) tooth whitening products (with chemical, bleaching action) with placebo or other comparable products.

- Full reports (either published or obtained from the investigators) had to be available for inclusion in the review.

- The application of tooth whitening products had to be exclusively carried out at home and outcome data had to be presented for tooth whitening, irrespective of the application time.

\section{Exclusion criteria}

- Quasi-randomised trials.

\section{Types of participants}

- Home-based whitening involving adults who were 18 years old and above were included in our review regardless of gender, race, profession, geographical location or baseline tooth shade. Because of issues related to compliance and ingestion of a bleaching agent in children and young adults, we decided to include trials including adults who were 18 years old and above.

- We included participants with teeth stained because of tetracycline use and smoking.

\section{Types of interventions}

\section{Inclusion criteria}

We considered any intervention including home-based chemicallyinduced whitening with the following comparisons:

- comparisons of different interventions (e.g. professional monitored versus over-the-counter; over-the-counter product $A$ versus $B$; professional monitored technique $A$ versus $B$ );

- intervention versus placebo or no treatment;

- comparisons between different concentrations;

- comparisons between different time periods of application.

\section{Exclusion criteria}

- Combination of in-office and home-based treatments were excluded.

- Home-based products having an abrasive action or physical removal of stains were excluded from the review (e.g. whitening dentifrices, whitening dental floss).

\section{Types of outcome measures}

\section{Primary outcomes}

Two primary outcomes were of interest.

\section{Tooth whitening - assessed by the dentist using any relevant tool}

The American Dental Association (ADA) acceptance programme guidelines for home-based tooth whitening products specify the 
use of two main methods to measure tooth colour in bleaching studies:

- value-oriented shade guides (subjective measurement); and

- electronic devices/colour measurement devices (objective measurement).

\section{Value-oriented shade guides}

Traditionally, visual colour determination is used based on visual comparison of tooth with colour standards (also called shade guides). The most common shade guides are Vitapan classical and its derivatives like Vitapan 3D master, tooth guide, bleached guide and linear guide. Ordinal scale ranging from 1 to 16 has been suggested by the manufacturer with 1 representing the lightest shade and 16 representing the darker shade. However, some authors considered 1 as the darkest and 16 as the lighter shade. Some studies reported a scale below 1 and beyond 16 when the tooth shade was lighter than the lightest shade tab and darker than the darkest shade.

Trubyte Bioform shade guide has also been used by some authors using a scale from 1 to 24 .

\section{Electronic devices/colour measurement devices}

Instruments for clinical shade matching encompass spectrophotometers, colorimeters and imaging systems. They provide a more objective measurement of whiteness compared to shade guides. For instrumental colour assessment of teeth the issue of suitable metric that corresponds to perpetual whiteness is important. Colourimeters, spectrophotometers, spectroradiometer and camera systems can allow computation of CIExyz or CIEL ${ }^{\star}{ }^{\star} b^{\star}$ values described by commission international deLE clariage (CIE 1978) an international standard for three dimensional colour space.

Using a calibration standard, red-green-blue values are determined and converted to $L^{*} a^{*} b^{*}$ values where $L^{*}$ represents the degree of lightness and $b^{\star}$ represents degree of yellowness. Tooth whitening is characterised by negative or decreased $b^{\star}$ values (reduction in yellowness) and positive or increased $L^{\star}$ values (increased lightness). A composite colour W is used by some and derived from individual $L^{*} a^{*} b^{\star}$ changes from baseline values. Some clinicians use $E^{\star}$ which indicates composite colour change irrespective of the direction of change.

In our review we considered the composite score represented by $W^{*}$ or $E^{\star}$ values wherever provided. In the absence of both, the value of $L^{*}$ is considered as it indicates a positive change towards increased lightness.

\section{Tooth whitening - reported by the patient using any relevant tool}

Improvement in tooth whitening as reported by the patient using any tool was considered in this review. Some authors reported visual analogue scale (VAS) from 0 to 10 to record patient acceptance with 0 indicating best acceptance and 10 indicating no acceptance, while some others used the scale where 0 represents least satisfaction and 10 represents most satisfaction.

Other scores used were on an ordinal scale of 0 to 3 where $0=$ no improvement in whiteness, 1 and $2=$ moderate improvement, and $3=$ improvement. In such cases, we combined 1,2 , and 3 as improvement in shade and counted them as events.

\section{Secondary outcomes}

1. Patient satisfaction or acceptability of the tooth whitening procedure (patient comfort).

2. Adverse effects: any side effects reported due to the bleaching procedure were considered in this review and described qualitatively.

3. Oral health-related quality of life.

\section{Search methods for identification of studies}

\section{Electronic searches}

Cochrane Oral Health's Information Specialist conducted systematic searches in the following databases for randomised controlled trials and controlled clinical trials. There were no language, publication year or publication status restrictions:

- Cochrane Oral Health's Trials Register (searched 12 June 2018) (Appendix 1);

- Cochrane Central Register of Controlled Trials (CENTRAL; 2018, Issue 6) in the Cochrane Library (searched 12 June 2018) (Appendix 2);

- MEDLINE Ovid (1946 to 12 June 2018) (Appendix 3);

- Embase Ovid (1980 to 12 June 2018) (Appendix 4).

Subject strategies were modelled on the search strategy designed for MEDLINE Ovid. Where appropriate, they were combined with subject strategy adaptations of the highly sensitive search strategy designed by Cochrane for identifying randomised controlled trials and controlled clinical trials as described in the Cochrane Handbook for Systematic Reviews of Interventions Chapter 6 (Lefebvre 2011).

\section{Searching other resources}

The following trial registries were searched for ongoing studies:

- US National Institutes of Health Ongoing Trials Register ClinicalTrials.gov (clinicaltrials.gov; searched 12 June 2018) (Appendix 5);

- World Health Organization International Clinical Trials Registry Platform (apps.who.int/trialsearch; searched 12 June 2018) (Appendix 6).

We searched the reference lists of included studies and relevant systematic reviews for further studies.

We did not perform a separate search for adverse effects of interventions used, we considered adverse effects described in included studies only.

\section{Data collection and analysis}

\section{Selection of studies}

Two review authors (Eachempati Prashanti (EP), Salian Kiran (SK)) independently screened the titles and abstracts from the electronic searches to identify potentially eligible studies. The search was designed to be sensitive and include controlled clinical trials, these were filtered out early in the selection process if they were not randomised. We obtained full-text copies of all potentially eligible studies and two pairs of review authors (EP and Ibrahim Ethem (IE), SK and Puneet Gupta (PG)) further evaluated in detail the studies for inclusion. We recorded the reasons why studies did not meet the inclusion criteria in the 'Characteristics of excluded 
studies' table. We resolved any disagreements by discussion. When resolution was not possible, we consulted the arbiter (Sumanth Kumbargere Nagraj (SKN)). Articles in languages other than English were assessed by their abstracts where possible and if they appeared to be potentially eligible, we obtained and translated the full-text article.

\section{Data extraction and management}

Two pairs of review authors (EP and IE, SK and PG) extracted the data independently, using a data extraction form specifically designed for this Cochrane Review. We resolved any disagreements by discussion. The two review authors independently checked data extraction forms obtained from translators and cross checked any doubtful aspects using Google translator. We entered all study details in the 'Characteristics of included studies' table in Review Manager 5 software (Review Manager 2014). We recorded the following details for each included trial.

- Publication details like year of publication and language.

- Demographic details of the report.

- Inclusion and exclusion criteria.

- Sample size.

- Method of randomisation.

- Allocation concealment.

- Blinding.

- Type of trial.

- Method of assessing the outcome, and dropouts if any.
- Type of intervention.

- Details of the outcome reported.

- Duration of follow-up.

- Results of the intervention.

- Funding details.

- Details about trials registration.

- For obtaining additional data and clarifications, we contacted the authors of the included and excluded trials via email.

\section{Assessment of risk of bias in included studies}

We independently assessed the risk of bias in the included trials for seven domains: sequence generation; allocation concealment; performance bias and detection bias; incomplete outcome data; selective outcome reporting; and other biases. For each of these components, we assigned a judgement regarding the risk of bias as either 'high', 'low' or 'unclear', based on guidance in section 8.5.d of the Cochrane Handbook for Systematic Reviews of Interventions (Higgins 2011). We contacted the trial authors if details were missing in the publications or were unclear. We resolved disagreements through consensus. We recorded our judgements and justifications in 'Risk of bias' tables for each included study and generated a 'Risk of bias' summary figure. We used these judgements while grading of the overall quality of evidence for outcomes in the 'Summary of findings' tables for each comparison.

We summarised the risk of bias according to Higgins 2011 as follows:

\begin{tabular}{llll}
\hline Risk of bias & Interpretation & In outcome & In included studies \\
\hline Low & $\begin{array}{l}\text { Plausible bias unlikely to seri- } \\
\text { ously alter the results }\end{array}$ & $\begin{array}{l}\text { Low risk of bias for all key } \\
\text { domains }\end{array}$ & $\begin{array}{l}\text { Most information is from studies at low risk of } \\
\text { bias }\end{array}$ \\
\hline Unclear & $\begin{array}{l}\text { Plausible bias that raises some } \\
\text { doubt about the results }\end{array}$ & $\begin{array}{l}\text { Unclear risk of bias for } \\
\text { one or more key domains }\end{array}$ & $\begin{array}{l}\text { Most information is from studies at low or un- } \\
\text { clear risk of bias }\end{array}$ \\
\hline High & $\begin{array}{l}\text { Plausible bias that seriously } \\
\text { weakens confidence in the re- } \\
\text { sults }\end{array}$ & $\begin{array}{l}\text { High risk of bias for one } \\
\text { or more key domains }\end{array}$ & $\begin{array}{l}\text { The proportion of information from studies at } \\
\text { high risk of bias is sufficient to affect the inter- } \\
\text { pretation of results }\end{array}$ \\
\hline
\end{tabular}

\section{Measures of treatment effect}

For dichotomous data, we used risk ratios (RRs), and for continuous data, we assessed the mean difference (MD) and presented results with $95 \%$ confidence intervals (Cls). For continuous data using different scales to measure the same primary outcome (improvement in tooth shade), we used the standardised mean difference (SMD). Change score and final score were combined in meta-analysis according to Higgins 2011 section 7.7.3.1. When multiple time points were given, longest follow-up time was considered (Higgins 2011, section 9.3.4).

\section{Unit of analysis issues}

For parallel-group and cluster-randomised studies, we used the individual as the unit of analysis. If clustered data were provided, we planned to adjust the standard errors of the estimates to take clustering into account (as outlined in section 16.3.4 of the
Cochrane Handbook for Systemic Reviews of Interventions (Higgins 2011)). For split-mouth studies, we used the quadrant of the mouth within an individual as a unit of analysis. For studies that have used a split-mouth design but reported data as a parallel-group study, we calculated the odds ratios using the Becker-Balagtas method, as outlined in Curtin 2002, using Stata software.

\section{Dealing with missing data}

We attempted to obtain missing data by contacting trial authors. If both mean and standard deviation were reported as graphs, we derived the data from the graphs by magnifying them and approximating the measures of mean and standard deviation. When mean and standard error (SE) were given, we calculated the standard deviation (SD) as given in Higgins 2011, section 7.7.3.3. When adjusted mean was given, we considered it in the analysis (Higgins 2011, section 9.2.3.2). When median and inter quartile 
range were given we used the data to calculate mean and SD. When mean and $P$ value were given, SD was calculated.

When data were presented as median (skewed data), we qualitatively described the results in the review.

\section{Assessment of heterogeneity}

We assessed heterogeneity by examining the forest plot to check for overlapping $\mathrm{Cls}$, using the $\mathrm{Chi}^{2}$ test for heterogeneity with a $10 \%$ level of significance to detect inconsistency in study results that were not due to random error (chance), and the $1^{2}$ statistic to denote the percentage of inconsistency in results due to intertrial variability that exceeded chance. We used the guidance given by the Cochrane Handbook for Systematic Reviews of Interventions to interpret the $1^{2}$ statistic: $0 \%$ to $40 \%$ as possibly insignificant, $30 \%$ to $60 \%$ as moderate heterogeneity, $50 \%$ to $90 \%$ as possibly substantial, and $75 \%$ to $100 \%$ as considerable heterogeneity depending on two factors: 1 . inconsistency in results was due to differences in the direction of effect estimates between trials rather than due to differences in the magnitude of effect estimates favouring an intervention; 2 . based on the strength of the evidence for heterogeneity from the $\mathrm{P}$ value for the $\mathrm{Chi}^{2}$ test for heterogeneity (Deeks 2011).

\section{Assessment of reporting biases}

We tested for publication bias using funnel plots and a formal test investigation of the degree of asymmetry using the method proposed by Egger 1997 wherever possible.

\section{Data synthesis}

We analysed the data using Review Manager 5 software (Review Manager 2014). We combined data available from trials with similar comparisons (same concentration and duration of application) and outcomes in the meta-analysis. We used standardised mean differences to combine continuous data as some trials used different scales. We used the random-effects model in the metaanalysis. If data were presented as adjusted mean and SE, we calculated SD from SE and considered the adjusted mean for analysis. If data were described in the form of ordinal outcomes, we converted the scale into dichotomous data by combining relevant adjacent categories and analysed using risk ratios and $95 \% \mathrm{Cl}$. When data were presented as odds ratios, log (odds ratio) was calculated based on the odds ratios and $95 \% \mathrm{Cl}$ given in the trial and the generic inverse variance method was applied. We calculated mean difference and standard error in split-mouth and cross-over trials and analysed the data using the generic inverse variance method.

\section{Subgroup analysis and investigation of heterogeneity}

We conducted subgroup analyses where there was heterogeneity.

To identify the reasons for clinical or methodological heterogeneity in meta-analyses, we carried out subgroup analyses, based on.

\section{Population}

- Age group of the patient.
- Baseline tooth shade.

\section{Method}

- Different concentrations.

- Varying application times and duration of treatment.

\section{Outcome measures}

- Subjective measurement involving shade guide.

- Objective measurements using electronic devices/instrument.

\section{Sensitivity analysis}

A sensitivity analysis is a repeat of the primary analysis or metaanalysis, substituting alternative decisions or ranges of values for decisions that were arbitrary or unclear. It involves undertaking the meta-analysis twice: first, including all studies and second, only including those that are definitely known to be eligible. Wherever feasible we did sensitivity analyses to assess the robustness of our findings by excluding data from trials at high risk and at unclear risk of bias.

\section{Summary of findings and assessment of the certainty of the evidence}

We used the GRADE approach to interpret findings (Schünemann 2011). We used GRADEpro GDT 2015 (GRADEpro GDT 2015) and imported data from Review Manager 5 (Review Manager 2014) to create 'Summary of findings' tables for the comparisons included in this review. The tables provide information concerning the overall certainty of the evidence at the outcome level, the magnitude of effect of the intervention examined and the sum of available data on the primary and secondary outcomes. The GRADE approach (Schünemann 2011) considers 'certainty' to be a judgement of the extent to which we can be confident that the estimates of effect are correct. Evidence from randomised controlled studies is initially graded as high and downgraded on each of five domains after full consideration of risk of bias, indirectness, imprecision, inconsistency and publication bias. A GRADE certainty level of 'high' reflects confidence that the true effect lies close to that of the estimate of the effect for an outcome. A judgement of 'moderate' certainty indicates that the true effect is likely to be close to the estimate of the effect, but acknowledges the possibility that it could be substantially different. 'Low' and 'very low' certainty evidence limit our confidence in the effect estimate (Balshem 2011).

\section{RES U L T S}

\section{Description of studies}

See Characteristics of included studies; Characteristics of excluded studies; and Characteristics of studies awaiting classification.

\section{Results of the search}

We included 71 trials (78 reports) in the review. (If the same study (one population) was separated into multiple reports we included the primary study and considered the rest as reports as per Higgins 2011.)

See Figure 1 for the selection process of search results. 
Figure 1. Study flow diagram.

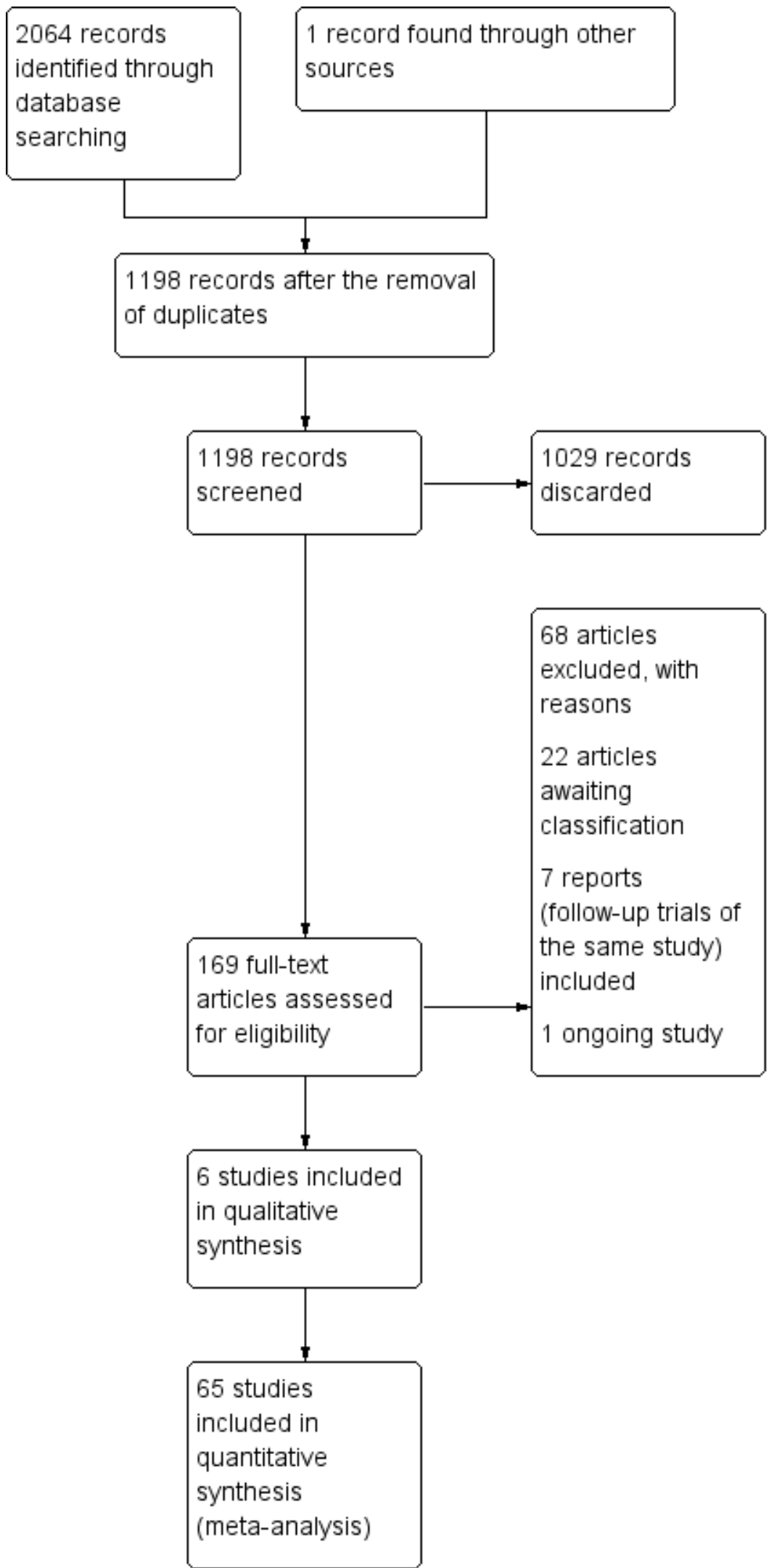




\section{Included studies}

See Characteristics of included studies for further details.

\section{Characteristics of trial settings and investigators}

Seventy studies were in the English language, one was in German (Auschill 2007).

The countries of origin for the included studies were.

- Forty-two from the USA (Kowitz 1994; Nathoo 1994; Russell 1996; Matis 1998; Cibirka 1999; Gerlach 2000; Kihn 2000; Kugel 2000; Matis 2000; Mokhlis 2000; Nathoo 2001; Gerlach 2002; Gerlach 2002a; Gerlach 2002b; Karpinia 2002; Kugel 2002; Nathoo 2002; Barlow 2003; Gerlach 2003; Li 2003; Myers 2003; Nathoo 2003; Biesbrock 2004; Garcia-Godoy 2004; Gerlach 2004; Gerlach 2004e; Hasturk 2004; Li 2004; Swift 2004; Cronin 2005; Gerlach 2005; Giniger 2005; Shahidi 2005; Matis 2006; Delgado 2007; Browning 2008; Gallo 2009; Papas 2009; Swift 2009; Bruhn 2012; Costa 2012; Oliveira 2013).

- Three trials were from Brazil (Mederios 2008; Meireles 2010; Kose 2011); five from Germany (Auschill 2007; Bizhang 2007; Krause 2008; Ziebolz 2008; Auschill 2012); four from Italy (Porciani 2006; Ferrari 2007; Porciani 2010; Navarra 2014); four from the UK (Brunton 2004; Collins 2004a; Walters 2004; Mohan 2008); three from Spain (Alonso 2006; Berga-Caballero 2006; Alonso 2014); two from Switzerland (Hannig 2007; Ziebolz 2007); two from Hong Kong (Wong 2004; Botelho 2017); one from China (Xu 2007); two from Turkey (Turkun 2010; Aka 2017); one from Japan (Tsubura 2005); one from Canada (Tam 2001); and one from Ireland (Hyland 2015).

- Sixty-one trials were parallel-design trials and seven were splitmouth (Matis 2000; Mokhlis 2000; Tsubura 2005; Alonso 2006; Matis 2006; Auschill 2007; Costa 2012); and three had a crossover design (Biesbrock 2004; Walters 2004; Porciani 2006).

- Twelve of the included trials had more than two groups for comparison. One trial had five groups (Browning 2008). Two trials, Alonso 2014 and Gerlach 2000, had four groups. The remaining nine trials had three groups for comparison (Li 2003; Li 2004; Wong 2004; Matis 2006; Bizhang 2007; Xu 2007; Krause 2008; Hyland 2015; Aka 2017). Seven trials included placebo in their comparisons (Wong 2004; Bizhang 2007; Xu 2007; Browning 2008; Krause 2008; Hyland 2015; Aka 2017). We included these seven trials separately in both the analysis testing bleaching agent versus placebo and bleaching agent versus bleaching agent. See Additional Table 1 for details.

- Out of the 71 trials, 54 provided grant information and out of these one was government funded (Navarra 2014). 53 were funded by a pharmaceutical company (Nathoo 1994; Cibirka 1999; Gerlach 2000; Kihn 2000; Kugel 2000; Matis 2000; Mokhlis 2000; Nathoo 2001; Gerlach 2002; Gerlach 2002a; Gerlach 2002b; Karpinia 2002; Kugel 2002; Nathoo 2002; Barlow 2003; Gerlach 2003; Li 2003; Myers 2003; Nathoo 2003; Biesbrock 2004; Brunton 2004; Collins 2004a; Garcia-Godoy 2004; Gerlach 2004; Gerlach 2004e; Hasturk 2004; Li 2004; Swift 2004; Walters 2004; Wong 2004; Cronin 2005; Gerlach 2005; Giniger 2005; Shahidi 2005; Tsubura 2005; Matis 2006; Porciani 2006; Bizhang 2007; Delgado 2007; Ferrari 2007; Hannig 2007; Xu 2007; Ziebolz 2007; Ziebolz 2008; Gallo 2009; Papas 2009; Swift 2009; Porciani 2010; Kose 2011; Auschill 2012; Costa 2012; Oliveira 2013; Hyland 2015).

- All the trials were carried out in a single centre.

\section{Characteristics of participants}

Forty-seven trials reported on both genders. The remaining 24 trials did not report on distribution of gender (Kowitz 1994; Nathoo 1994; Matis 1998; Cibirka 1999; Kihn 2000; Mokhlis 2000; Nathoo 2001; Nathoo 2002; Gerlach 2003; Myers 2003; Brunton 2004; Garcia-Godoy 2004; Walters 2004; Berga-Caballero 2006; Auschill 2007; Browning 2008; Krause 2008; Gallo 2009; Turkun 2010; Kose 2011; Bruhn 2012; Alonso 2014; Navarra 2014; Hyland 2015). The minimum age included in a study was 18 years and the maximum age included in a study was 79 years (Russell 1996). The minimum sample size was six (Berga-Caballero 2006) and the maximum sample size was 117 (Collins 2004a) with a median value of 58 .

Thirty-one trials reported on the minimum baseline shade for inclusion in the trial. Twelve reported $A 2$ as the baseline shade (Kugel 2000; Karpinia 2002; Gerlach 2003; Garcia-Godoy 2004; Wong 2004; Cronin 2005; Hannig 2007; Ziebolz 2007; Ziebolz 2008; Papas 2009; Kose 2011; Oliveira 2013), 14 reported A3 as the baseline shade (Nathoo 1994; Kihn 2000; Nathoo 2001; Nathoo 2002; Nathoo 2003; Li 2003; Brunton 2004; Li 2004; Giniger 2005; Auschill 2007; Delgado 2007; Browning 2008; Mohan 2008; Auschill 2012), and three reported C1 (Matis 2006; Meireles 2010; Turkun 2010) based on Vita shade guide. Two studies used Trubyte shade guide; one used B85 as the baseline shade (Mokhlis 2000) while B65 was used in the other (Gallo 2009).

Two trials compared the effects of bleaching agent on participants with tetracycline stains (Kugel 2002; Matis 2006). One trial discussed the effect of dietary habits of participants on tooth whitening (Meireles 2010). One trial compared the effect of tooth whitening between smokers and non-smokers (Porciani 2006).

\section{Characteristics of interventions}

We divided the 71 trials included in our review into two categories.

\section{Bleaching agent versus placebo}

Twenty-six trials were included in this group. Among the 26 studies, the following comparisons were identified.

- Six trials comparing carbamide peroxide (CP) gel in tray versus placebo.

- Two trials comparing hydrogen peroxide (HP) gel in tray versus placebo.

- Ten trials comparing HP strips versus placebo.

- One trial comparing CP paint-on gel versus placebo.

- Two trials comparing HP paint-on gel versus placebo.

- Three trials comparing sodium hexametaphosphate (SHMP) chewing gum versus placebo.

- One trial comparing sodium tripolyphosphate (STPP) chewing gum versus placebo.

- One trial comparing HP mouthwash versus placebo.

\section{1a. CP gel in tray versus placebo}

Six trials were included in this comparison.

Hyland 2015, a three-arm study compared $10 \%$ CP and 5\% CP applied 2 hours a day for 2 weeks to the placebo gel. The CP formulation in this trial contained sodium tripolyphosphate. We compared $5 \% \mathrm{CP}$ to placebo gel. 
Browning 2008 used five interventions in his multiarm study. We compared $10 \% \mathrm{CP}+0.5 \%$ potassium nitrate $(\mathrm{KNO} 3)+0.25 \%$ sodium fluoride $(\mathrm{NaF})$ to a placebo gel, applied for 11 weeks.

A three-arm trial conducted by Aka 2017 used 6\% HP, 10\% CP and placebo. Analysis in this trial was done at tooth level. The study authors divided the groups based on the tooth shade as light, medium dark and dark. 10\% CP gel was compared to placebo in our meta-analysis, which was applied for 8 hours to 10 hours daily for 14 days. For the control group, no bleaching agent was applied.

Russell 1996 and Matis 1998 used 10\% CP and used it overnight for 2 weeks with a follow-up of 6 months.

Mederios 2008 used 10\% CP overnight for 3 weeks.

\section{1b. HP gel in tray versus placebo}

Mohan 2008 and Myers 2003 studied the effect of $6 \%$ and 3\% HP gel respectively which was applied twice daily for 14 days.

\section{1c. HP strips versus placebo}

Ten trials, studied the effect of HP strips versus placebo. All trials included HP strips with varying concentrations.

Gerlach $2004 \mathrm{e}$ used 10\% HP, twice a day for 30 minutes for 1 week and 2 weeks respectively.

$6 \%$ HP was used twice a day for 30 minutes in one multiarm trial Bizhang 2007.

Kugel 2000 used 5.3\% HP, twice daily for 30 minutes each time for 2 weeks.

$6 \% \mathrm{HP}$ was used twice a day for 30 minutes in Swift 2009 for 2 weeks and 6 weeks.

Wong 2004 a multiarm trial, compared 6\% HP strip, 18\% CP painton gel and placebo. Data for $6 \%$ HP strip versus placebo were used in this meta-analysis. Strips were used twice a day for 30 minutes, for 14 days.

Papas 2009 used 10\% HP, twice a day for 30 minutes for 1 week and 2 weeks.

Swift 2004 and Garcia-Godoy 2004 compared 14\% HP strips applied twice daily for 30 minutes for 3 weeks and 6 weeks respectively.

Gerlach 2002 used 5.3\% HP, twice daily for 30 minutes each time for 2 weeks with a follow-up of 6 months.

Bruhn 2012 used a HP gel twice a day for 3 weeks and reported on patient-reported satisfaction and oral health-related quality of life.

\section{1d. CP paint-on gel versus placebo}

One trial Nathoo 2002 compared the effect of CP paint-on gel with placebo.

Nathoo 2002 studied 18\% CP versus placebo gel, which was applied immediately after brushing and subjects were instructed to keep their mouth open for 30 seconds after application. They refrained from eating and drinking for 30 minutes after application.

\section{1e. HP paint-on gel versus placebo}

Two trials compared the effect of HP paint-on gel with control gel.
Xu 2007, a multiarm trial, and Collins 2004a compared 6\% and 5.9\% HP gel applied twice daily for 2 weeks respectively. Collins also evaluated colour change at 1 week.

\section{1f. SHMP chewing gum versus placebo}

Three trials compared SHMP chewing gum to placebo.

Biesbrock 2004, a cross-over study used $7.5 \%$ SHMP on 19 subjects each (period A) and 18 subjects each (period B) for 5 minutes daily for 2 days followed by 60 seconds rinse with tea. This was repeated approximately 8 times a day. Outcomes looked into were stain prevention and removal. Placebo group were given a negative control chewing gum.

Porciani 2006 was another cross-over study among smokers and non-smokers, using 4\% SHMP on 54 subjects each in placebo and experimental groups. The subjects chewed chewing gum 4 times a day for 12 weeks. Placebo group received no gum.

Walters 2004 compared 5.6\% SHMP to a negative control chewing gum in a 3-day cross-over trial separated by a 10-day wash out period including 10 subjects in each group.

\section{1g. STPP chewing gum versus placebo}

Sodium tripolyphosphate was used in a study by Porciani 2010, including smokers and habitual tea users in each group. They were asked to chew the gum 3 times a day for 10 minutes for 6 weeks and compared to the placebo group who received a control chewing gum.

\section{1h. HP mouthwash versus placebo}

One trial (Hasturk 2004) compared 1.5\% fluoridated HP-based mouthrinse to placebo. Mouthrinse was used twice daily for 30 seconds for 6 months.

\section{Bleaching agent versus bleaching agent}

Fifty-one trials were included in this group among which six are multiarm trials which were also included in the comparison of bleaching agent versus placebo.

Among these, the following comparisons were made.

- Eighteen trials compared CP tray versus CP tray.

- Seven trials compared CP tray versus HP tray.

- Ten trials compared HP strips versus CP tray.

- Two trials compared HP strips versus HP tray.

- Two trials compared HP strips versus HP strips.

- One trial compared HP strip versus HP mouthwash.

- Two trials compared CP paint-on versus HP strip.

- Two trials compared HP paint-on versus HP strip.

- One trial compared SPC paint-on versus HP strip.

- Two trials compared CP paint-on versus CP paint-on.

- One trial compared CP paint-on versus HP paint-on.

- One trial compared HP paint-on versus HP paint-on.

- One trial compared sodium percarbonate (SPC) paint-on versus CP paint-on.

- One trials compared SPC paint-on versus HP paint-on. 


\section{2a. CP tray versus CP tray}

A total of 18 trials are included comparing $C P$ in a tray versus $C P$ in a tray.

Nathoo 2001 used an overnight application of 5\% and 10\% CP ( 6 to 8 hours per day) for 1 week. Hyland 2015 used the same concentrations of bleaching agent for 2 hours daily for 2 weeks.

Four trials (Kowitz 1994; Nathoo 1994; Cibirka 1999; Tsubura 2005) reported on the effect of 10\% CP of different brands. Tsubura 2005 and Cibirka 1999 used the gel in tray overnight for 2 weeks while Kowitz 1994 used it for 3 hours or more for 2 weeks. Nathoo 1994 applied the gel twice daily for 30 minutes, for a duration of 2 weeks.

Turkun 2010 reported on effects of 10\% CP and 28\% CP with an application time of 8 hours overnight and 20 minutes per day over a 2-week period.

Meireles 2010 had 46 and 45 subjects in experimental and control groups respectively and applied the whitening agents 2 hours a day for 2 weeks. He followed the same regimen for 3 weeks with 45 and 44 participants in $10 \%$ and $16 \%$ CP groups respectively. He did a follow-up study including 45 and 44 participants for 1 year and 42 and 39 participants for 2 years respectively, following the same application protocol.

Gallo 2009 reported a trial of 10 days duration with $30 \%$ CP with and without potassium nitrate. The application time was 1 hour per day.

Kose 2011 reported the effect of bleaching with 16\% CP with and without potassium nitrate and sodium fluoride. The bleaching was done for 2 weeks with 6 hours application time.

Giniger 2005 is a 5-year follow-up trial comparing the effect of $16 \%$ $\mathrm{CP}(\mathrm{n}=13)$ versus $16 \%$ CP with amorphous calcium phosphate $(\mathrm{n}=$ 14). The application time was 3 hours per day over 2 -week period.

Matis 2006 is a follow-up trial for 180 days continued from a splitmouth trial with 40 and 39 participants in experimental and control groups on tetracycline stained teeth with overnight application using $10 \%$ CP and 15\% CP over 6 months.

Krause 2008 conducted a trial on $10 \%$ CP and 17\% CP with 2 hours a day application over a period of 2 weeks.

Matis 2000 and Kihn 2000 reported the effect of overnight application of $10 \%$ CP and $16 \%$ CP for 2 weeks.

Two trials, Navarra 2014 ( $\mathrm{n}=10$ per group) and Browning 2008 ( $n=19$ per group), reported on the effect of $10 \%$ CP with and without potassium nitrate and sodium fluoride applied overnight for 2 weeks.

Tam 2001 reported a trial with 10\% CP and 10\% CP without potassium nitrate with overnight application time for 2 weeks.

\section{2b. CP tray versus HP tray}

Ziebolz 2007 studied the effect of $20 \%$ CP versus $7.5 \%$ HP. He used the bleaching gel for 4 hours a day for $20 \%$ CP group and 30 minutes a day for 7.5\% HP group for 12 days. Mokhlis 2000 used the same concentration but applied the gel twice daily for an hour over a period of 12 weeks.
Alonso 2014 reported a 2-week trial with an application time of 1 hour per day using $10 \% \mathrm{CP}$ and $7.5 \% \mathrm{HP}$.

Delgado 2007 reported on application time of 30 minutes per day over 2 weeks for $20 \% \mathrm{CP}$ and $9 \% \mathrm{HP}$.

Aka 2017 reported a multiarm trial at tooth level, comparing 10\% $\mathrm{CP}$ and $6 \% \mathrm{HP}$. The application time was 8 hours to 10 hours per day over 2 weeks for CP group.

Berga-Caballero 2006 reported a trial with 3.5\% HP and 10\% CP. The application time was 3 hours daily for 24 days for $3.5 \%$ HP group and 2 hours daily for 28 days in $10 \%$ CP group.

Alonso 2006 conducted a split-mouth study on the effect of 3.5\% HP and $5 \%$ potassium nitrate over $10 \% \mathrm{CP}$ for 4 weeks. The application time was 3 hours per day.

\section{2c. HP strips versus CP tray}

Ten trials compared HP strips to $\mathrm{CP}$ gel in tray in varying concentrations and application times.

Gerlach 2002b used 5\% CP gel with 5\% potassium nitrate in tray and compared it to $6 \%$ HP strips used for 30 minutes, twice daily for 7 days.

Gerlach 2000 a multiarm trial; Gerlach 2002a; Hannig 2007; and Karpinia 2002 compared $10 \%$ CP gel in tray versus $5.3 \%$ or $6 \%$ or $6.5 \%$ HP strips for 2 weeks. Gerlach 2002b applied the strips twice daily for 30 minutes and gel in tray for 2 hours a day, both for a duration of 2 weeks. Gerlach 2002a applied strips twice a day for 1 hour and the tray for 2 hours once daily for 2 weeks. Hannig 2007 followed the same application protocol for strips as mentioned above, however, the tray was used once daily for 1 hour over 2 weeks. Karpinia 2002 applied whitening strips for 30 minutes, twice daily and whitening gel in tray for 2 hours daily.

Costa 2012 compared 35\% CP gel in tray to 14\% HP strips in a splitmouth trial. Both interventions were applied simultaneously for 30 minutes, twice a day for 2 weeks and the application times were separated by 3 hours.

Ferrari 2007 used $6 \%$ HP versus 10\% CP for 6 weeks. He applied the strips for 30 minutes, twice daily and gel in tray for 2 hours daily.

Kugel 2002 also followed the same regimen as Ferrari 2007 for a duration of 2 months.

Botelho 2017 used $6.5 \%$ HP strips versus 16\% CP in tray with 13 and 11 participants allocated to each group respectively. The subjects wore tray with the gel for up to 2 hours or overnight during the 3month trial. Strip group applied the strips onto the labial surfaces of the teeth twice daily for 30 minutes for 3 months.

Li 2003 a multiarm trial compared 6.5\% HP strips to 7.5\% HP in tray and $16 \%$ CP in tray. Strips were applied twice a day for 30 minutes and tray was used overnight, both for 21 days.

\section{2d. HP strips versus HP tray}

Two trials compared HP strips to HP gel in tray.

Gerlach 2004 compared 14\% HP strips used for 21 days and 9.5\% HP in custom tray used for 9 days. Both the groups applied the bleaching agent twice a day for 30 minutes. 
Auschill 2012 studied 5\% HP in tray and 5.3\% HP strips for 30 minutes, twice daily for 14 consecutive days.

\section{2e. HP strip versus HP strip}

Oliveira 2013 compared 9.5\% high adhesion HP strips to marketed $10 \%$ control strip for 8 days. $9.5 \%$ HP strips were applied for 2 hours once daily and control strips were applied for 30 minutes once a day.

Shahidi 2005 studied 10\% HP strip with very thin 6\% HP gel $(0.12$ $\mathrm{mm}$ strip) versus $6 \% \mathrm{HP}$ gel $(0.2 \mathrm{~mm})$ applied for 30 minutes, twice daily for 14 days.

\section{2f. HP strip versus HP mouthwash}

One trial (Gerlach 2005) compared two HP tooth whitening systems including 2\% HP pre-rinse and 10\% HP strips. Pre-rinse group was instructed to rinse twice daily with $15 \mathrm{ml}$ solution for 60 seconds before brushing. The strip group were specified twice daily application for 30 minutes.

\section{2g. CP paint-on gel versus HP strip}

Two trials compared HP strips to CP paint-on gel (Wong 2004; Cronin 2005).

Both trials compared $6 \%$ HP strips to $18 \%$ CP paint-on gel. In both trials strips were used twice daily for 2 weeks. Paint-on was used twice daily for 30 minutes for 2 weeks in Cronin 2005 and 15 minutes in Wong 2004.

\section{2h. HP paint-on gel versus HP strip}

Two trials compared $5.9 \%$ or $6 \%$ HP strips to $5.9 \%$ HP paint-on gel (Auschill 2007; Xu 2007). In Xu 2007 a multiarm trial, both the groups applied the bleaching agent twice daily for 1 week. Auschill 2007 used strips twice daily for 30 minutes and paint-on gel twice daily for 15 minutes.

\section{2i. SPC paint-on versus HP strip}

One trial compared HP strips to sodium percarbonate (Bizhang 2007).

Bizhang 2007 compared 6\% HP strips to 19\% SPC. Strips were instructed to be applied twice daily for 30 minutes over a 14-day period. Paint-on gel was applied to the facial surfaces of the teeth for 14 days.

\section{2j. CP paint-on versus CP paint-on}

Two trials were included in this comparison.

Li 2004 a multiarm trial with 120 participants balanced equally into three groups, used $18 \%$ CP with different application times (twice $2 x$, thrice $3 x, 4$ times $4 x$ per day). In the $2 x$ group, no air drying was used and participants were asked not to eat and drink for 15 minutes after the gel was applied. In the $4 x$ group, 30 seconds air drying and 30 minutes refraining from eating and drinking was advocated.

Brunton 2004 compared $18 \%$ CP gel and $16.4 \%$ CP gel, applied twice a day for 30 seconds each for 2 weeks. Subjects were asked to refrain from eating and drinking for 30 minutes.

\section{2k. CP paint-on versus HP paint-on}

One trial (Nathoo 2003) compared $8.7 \%$ HP versus $25 \% \mathrm{CP}$, where a thin layer of gel was applied one tooth at a time and subjects were instructed not to rinse, eat or drink for 15 minutes. This was repeated 3 times a day for 2 weeks.

\section{HP paint-on versus HP paint-on}

One trial (Ziebolz 2008) studied 6\% HP after potassium fluoride application versus 6\% HP without desensitiser application. Application was done twice a day for 10 minutes for 7 days.

\section{$2 \mathrm{~m}$. SPC paint-on versus CP paint-on}

Barlow 2003 compared 19\% SPC and 18\% CP gel twice a day for 2 weeks.

\section{2n. SPC paint-on versus HP paint-on}

Gerlach 2003 compared 19\% SPC and 8.7\% HP. Both groups advocated application of a thin layer of gel after drying the tooth at night. They were instructed not to eat or drink after application and to brush normally the next morning.

\section{Outcomes reported in the trials}

\section{Primary outcomes}

Sixty-nine trials studied improvement in tooth whitening as assessed by the dentist using any relevant scale. 11 trials reported improvement in tooth whitening based on patient's satisfaction levels (Kowitz 1994; Tam 2001; Wong 2004; Matis 2006; Hannig 2007; Krause 2008; Mederios 2008; Meireles 2010; Bruhn 2012; Costa 2012; Aka 2017).

\section{Secondary outcomes}

Eight trials gave patient-reported level of comfort with the treatment (Kugel 2002; Nathoo 2002; Wong 2004; Ziebolz 2007; Ziebolz 2008; Meireles 2010; Auschill 2012; Costa 2012).

Fourteen trials did not report on any adverse reaction after tooth whitening (Nathoo 1994; Cibirka 1999; Russell 1996; Nathoo 2002; Gerlach 2003; Nathoo 2003; Biesbrock 2004; Collins 2004a; Hasturk 2004; Walters 2004; Porciani 2006; Mohan 2008; Meireles 2010; Porciani 2010).

Meireles 2010 in one of his 2-year follow-up reports studied only oral health-related quality of life as an outcome. Two trials have reported oral health-related quality of life along with other outcomes (Wong 2004; Bruhn 2012).

\section{Excluded studies}

See Characteristics of excluded studies tables for further details. 22 articles were excluded as they were abstracts of conference presentations (Andreana 2000; Dickinson 2000; Browning 2001; Donly 2001; Godson 2001; Sagel 2001; Smith 2001; Swift 2001; Gerlach 2002d; Lee 2003; Amini 2009; Auschill 2009; Lisante 2009; Anastasia 2010; Archila 2010; Amini 2011; Majeed 2011; Simon 2011; Walter 2011; Garcia-Godoy 2012; Mazur 2013; Perdigao 2013).

We procured 46 full-text articles and excluded them for the following reasons. 
- Six trials included studies with in-office bleaching (Burgio 2001; Matis 2005; Zantner 2006; Martin 2015; NCT02603354; NCT02682329).

- Nine trials included children or adolescents in their study (Tam 1999; Donly 2002; Donly 2002a; Loyola-Rodriguez 2003; Gerlach 2004d; Cardoso 2010; Corby 2014; Pinto 2014; Pinto 2017).

- Five trials included studies, which used mechanical method of stain removal like toothbrushing, and whitening dentifrices (Simon 2001; Gerlach 2002c; Gerlach 2003a; Karpinia 2003; Gerlach 2004a).

- One trial reported on home bleaching in which the agent was applied by a professional (Farrell 2006).

- One trial reported the use of a non-whitening chewing gum without an active ingredient (Yankell 1997).

- Of the remaining 24 trials.

- Nine trials reported only on effects on oral tissues or associated tooth sensitivity or both or were controlled clinical trials (Schulte 1993; Curtis 1996; Jorgensen 2002; Leonard 2002; Collins 2004; Leonard 2007; Farrell 2008; de Geus 2015a; de Geus 2015b).

- Three trials reported the effects of whitening agents on pulp (Schulte 1994; Fugaro 2004; Fugaro 2005).

- Four trials reported on the dilution kinetics of whitening agents (Matis 1999; Matis 2002; Gerlach 2004c; Marques 2012).

- Two studies reported on plaque retention post-bleaching (Schiff 1994; Gursoy 2008).

- One study reported on oral microflora (Alkmin 2005).

- One study reported on the effect of a desensitising agent (Leonard 2004).
- One study reported on the effect of coffee exposure on bleaching (Rezende 2013).

- One study reported the effect of bleaching on orthodontic brackets (Jadad 2011).

- One study reported the effect of two different tray designs used during bleaching (Matis 2002a).

- One study reported the efficacy of using a chromameter to assess bleaching (Gerlach 2002e).

\section{Studies awaiting classification}

See Characteristics of Characteristics of studies awaiting classification tables for further details.

- Twelve studies had incomplete or missing data; hence, we could not use them for analysis (Gegauff 1993; Reinhardt 1993; Rosenstiel 1996; Barnes 1998; Pohjola 2002; Ozcan 2003; Browning 2004; Ferrari 2004; Gambarini 2004; Braun 2007; Shin 2010; Simon 2014).

- We are awaiting full texts for eight published trials (Heymann 1998; Sielski 2003; Gerlach 2004b; Guerrero 2007; Bizhang 2017; Kim 2018; Maran 2018; Rossi 2018).

- Two studies were protocol registration of completed studies, but we could not access the full text (NCT02151058; NCT03217994).

\section{Ongoing studies}

- One clinical trial has not yet published the results and is ongoing (NCT03026725).

\section{Risk of bias in included studies}

See Figure 2 for details. 
Figure 2. Risk of bias summary: review authors' judgements about each risk of bias item for each included study.

\begin{tabular}{|c|c|c|c|c|c|c|c|}
\hline & 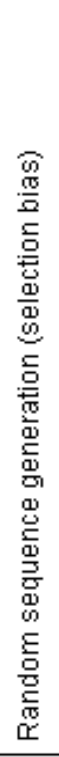 & 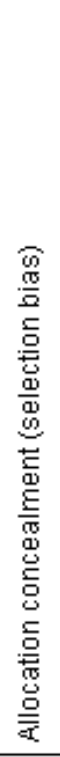 & 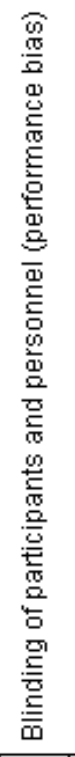 & 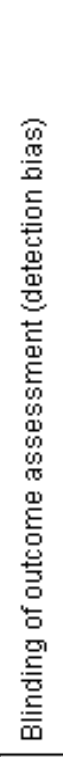 & 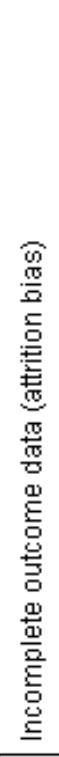 & 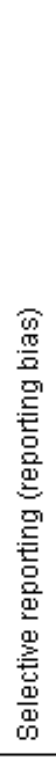 & 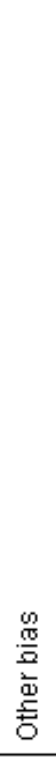 \\
\hline Aka 2017 & + & $?$ & $?$ & $\odot$ & $\oplus$ & + & + \\
\hline Alonso 2006 & $?$ & $?$ & $?$ & $?$ & + & + & + \\
\hline Alonso 2014 & + & $?$ & $?$ & $?$ & + & + & + \\
\hline Auschill 2007 & $?$ & $?$ & $?$ & $?$ & + & + & + \\
\hline Auschill 2012 & $\odot$ & $?$ & $?$ & + & + & + & + \\
\hline Barlow 2003 & $?$ & $?$ & 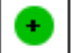 & $?$ & + & + & + \\
\hline Berga-Caballero 2006 & $?$ & $?$ & $?$ & $?$ & + & + & + \\
\hline Biesbrock 2004 & $?$ & $?$ & $?$ & $?$ & + & + & + \\
\hline Bizhang 2007 & $?$ & $?$ & + & + & + & + & + \\
\hline Botelho 2017 & + & ? & ? & $?$ & + & + & + \\
\hline Browning 2008 & $?$ & $?$ & $?$ & $?$ & + & + & + \\
\hline Bruhn 2012 & $?$ & ? & $?$ & + & $?$ & + & + \\
\hline Brunton 2004 & $?$ & $?$ & $?$ & $?$ & + & + & + \\
\hline Cibirka 1999 & + & ? & $\odot$ & $?$ & ? & + & + \\
\hline Collins 2004a & ? & $?$ & $?$ & $?$ & + & + & + \\
\hline Costa 2012 & ? & $?$ & $?$ & $?$ & + & + & + \\
\hline Cronin 2005 & $?$ & $?$ & $?$ & $?$ & + & + & + \\
\hline Delgado 2007 & + & $?$ & $?$ & $?$ & $?$ & + & + \\
\hline Ferrari 2007 & $?$ & $?$ & + & $?$ & + & + & + \\
\hline Gallo 2009 & $?$ & $?$ & $?$ & $?$ & + & + & + \\
\hline
\end{tabular}


Figure 2. (Continued)

\begin{tabular}{|c|c|c|c|c|c|c|c|}
\hline Gallo 2009 & $?$ & $?$ & $?$ & $?$ & $\odot$ & $\odot$ & + \\
\hline Garcia-Godoy 2004 & ? & $?$ & $?$ & $?$ & + & $\odot$ & + \\
\hline Gerlach 2000 & $?$ & $?$ & $?$ & $?$ & $\oplus$ & + & + \\
\hline Gerlach 2002 & $?$ & $?$ & + & $?$ & + & + & + \\
\hline Gerlach 2002a & $?$ & $?$ & $\odot$ & $?$ & + & + & + \\
\hline Gerlach 2002b & $?$ & $?$ & $?$ & $?$ & + & $\odot$ & + \\
\hline Gerlach 2003 & $?$ & $?$ & $\odot$ & $?$ & + & + & + \\
\hline Gerlach 2004 & $?$ & $?$ & + & ? & + & $\odot$ & + \\
\hline Gerlach 2004e & $?$ & $?$ & $\odot$ & $?$ & + & + & + \\
\hline Gerlach 2005 & ? & ? & ? & ? & + & + & + \\
\hline Giniger 2005 & + & + & + & + & + & + & + \\
\hline Hannig 2007 & $?$ & $?$ & $?$ & $?$ & + & + & + \\
\hline Hasturk 2004 & + & + & + & + & ? & + & + \\
\hline Hyland 2015 & ? & ? & ? & ? & + & + & + \\
\hline Karpinia 2002 & + & $?$ & + & $?$ & + & + & + \\
\hline Kihn 2000 & $\odot$ & $?$ & $?$ & $?$ & + & + & + \\
\hline Kose 2011 & + & ? & + & + & + & + & + \\
\hline Kowitz 1994 & $?$ & + & + & ? & + & + & + \\
\hline Krause 2008 & + & $?$ & + & + & + & + & + \\
\hline Kugel 2000 & $\odot$ & ? & + & $\odot$ & + & + & + \\
\hline Kugel 2002 & $?$ & ? & $\odot$ & $?$ & + & + & + \\
\hline Li 2003 & + & + & + & $\odot$ & + & + & + \\
\hline Li 2004 & $?$ & $?$ & $?$ & ? & + & + & + \\
\hline Matis 1998 & ? & ? & $\odot$ & $\odot$ & + & + & + \\
\hline Matis 2000 & $?$ & $?$ & $?$ & $?$ & + & + & + \\
\hline Matis 2006 & $?$ & $?$ & $?$ & $?$ & + & + & + \\
\hline Mederios 2008 & + & ? & + & $?$ & + & + & + \\
\hline Meireles 2010 & + & $?$ & $\odot$ & $?$ & + & + & + \\
\hline Mohan 2008 & $?$ & $?$ & $?$ & $?$ & + & + & + \\
\hline Mokhlis 2000 & + & + & $?$ & $?$ & + & + & + \\
\hline Myer & + & + & $?$ & $?$ & + & + & + \\
\hline
\end{tabular}


Figure 2. (Continued)

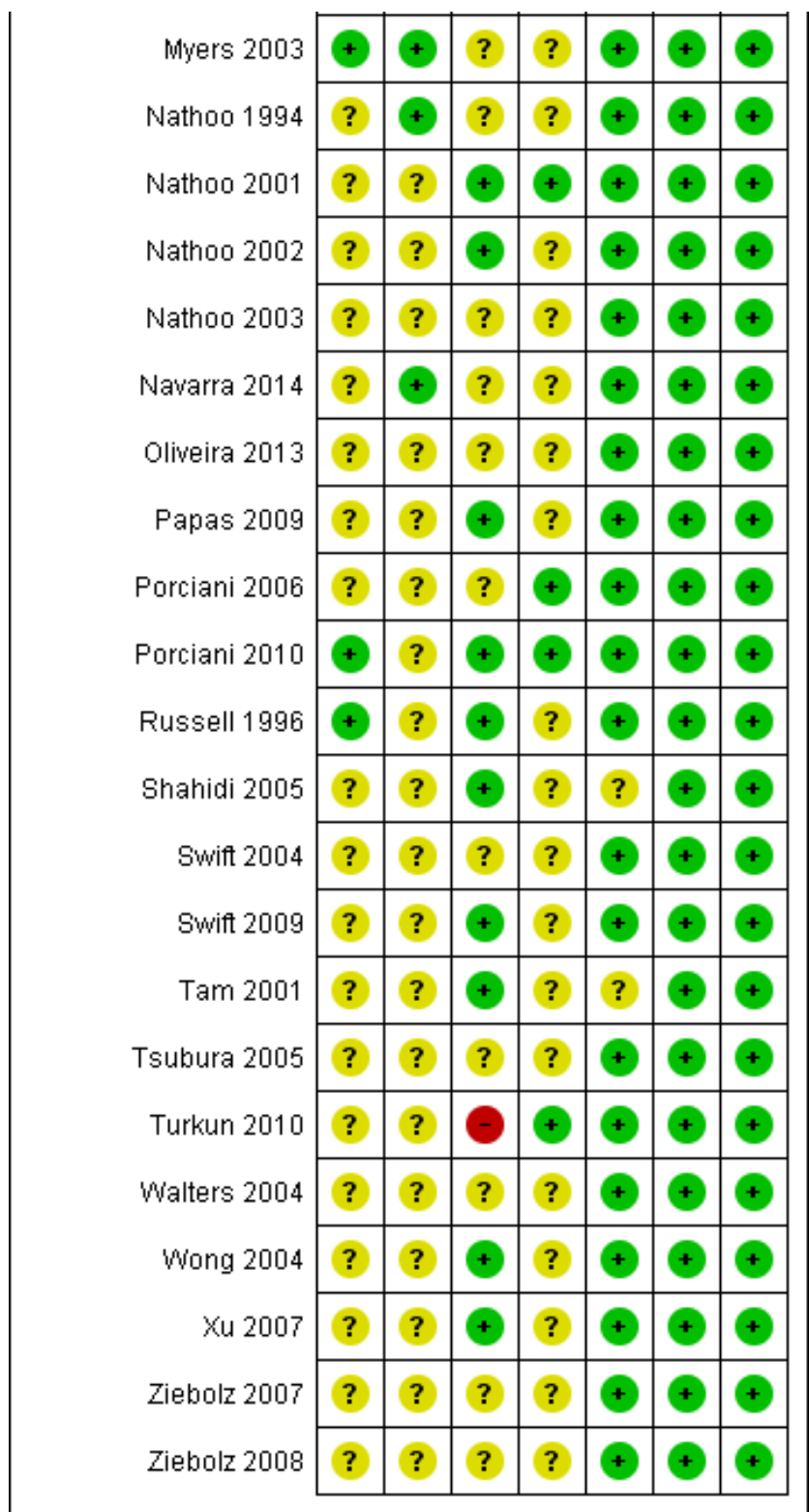

\section{Allocation}

Twenty of the included trials reported the method of sequence generation and were at a low risk of bias (Russell 1996; Cibirka 1999; Kihn 2000; Mokhlis 2000; Karpinia 2002; Kugel 2002; Li 2003; Myers 2003; Hasturk 2004; Giniger 2005; Delgado 2007; Krause 2008; Mederios 2008; Meireles 2010; Porciani 2010; Kose 2011; Auschill 2012; Alonso 2014; Aka 2017; Botelho 2017); the remaining trials were at an unclear risk of bias.

Eight of the included studies reported concealment of allocation (Kowitz 1994; Nathoo 1994; Mokhlis 2000; Li 2003; Myers 2003; Hasturk 2004; Giniger 2005; Navarra 2014). The rest of the trials were marked unclear.

\section{Blinding}

Out of 71 included trials, blinding of participants and personnel was unclear in 39 trials (Nathoo 1994; Gerlach 2000; Kihn 2000;
Matis 2000; Mokhlis 2000; Gerlach 2002b; Myers 2003; Nathoo 2003; Biesbrock 2004; Brunton 2004; Collins 2004a; Garcia-Godoy 2004; Li 2004; Swift 2004; Walters 2004; Cronin 2005; Gerlach 2005; Tsubura 2005; Alonso 2006; Berga-Caballero 2006; Matis 2006; Porciani 2006; Auschill 2007; Delgado 2007; Hannig 2007; Ziebolz 2007; Browning 2008; Mohan 2008; Ziebolz 2008; Gallo 2009; Auschill 2012; Bruhn 2012; Costa 2012; Oliveira 2013; Alonso 2014; Navarra 2014; Hyland 2015; Aka 2017; Botelho 2017) and high in one (Turkun 2010); 31 trials had reported satisfactory blinding of participants and personnel.

Blinding of assessors was reported in 14 trials and were at a low risk of detection bias (Matis 1998; Kugel 2000; Nathoo 2001; Kugel 2002; Li 2003; Hasturk 2004; Giniger 2005; Porciani 2006; Bizhang 2007; Krause 2008; Porciani 2010; Turkun 2010; Auschill 2012; Bruhn 2012). 
Aka 2017 was at a high risk of detection bias due to lack of clarity of how teeth were selected for outcome assessment.

The other 56 were marked unclear as the trials described their studies as 'double-blinded', but no details of assessor blinding were given, or were single-blinded studies (Kowitz 1994; Nathoo 1994; Russell 1996; Cibirka 1999; Gerlach 2000; Kihn 2000; Matis 2000; Mokhlis 2000; Tam 2001; Gerlach 2002; Gerlach 2002a; Gerlach 2002b; Karpinia 2002; Nathoo 2002; Barlow 2003; Gerlach 2003; Myers 2003; Nathoo 2003; Biesbrock 2004; Brunton 2004; Collins 2004a; Garcia-Godoy 2004; Gerlach 2004; Gerlach 2004e; Li 2004; Swift 2004; Walters 2004; Wong 2004; Cronin 2005; Gerlach 2005; Shahidi 2005; Tsubura 2005; Alonso 2006; Berga-Caballero 2006; Matis 2006; Auschill 2007; Delgado 2007; Hannig 2007; Ferrari 2007; Xu 2007; Ziebolz 2007; Browning 2008; Mederios 2008; Mohan 2008; Ziebolz 2008; Gallo 2009; Papas 2009; Swift 2009; Meireles 2010; Kose 2011; Costa 2012; Oliveira 2013; Alonso 2014; Navarra 2014; Hyland 2015; Botelho 2017).

\section{Incomplete outcome data}

Six trials (Cibirka 1999; Tam 2001; Hasturk 2004; Shahidi 2005; Delgado 2007; Bruhn 2012) were marked unclear for risk of attrition bias as the trials did not mention any details about dropouts and there was a mismatch between the number randomised and number analysed.

\section{Selective reporting}

All 71 trials were at a low risk of bias for selective reporting.

\section{Other potential sources of bias}

All 71 trials were at a low risk for other bias.

\section{Overall risk of bias}

Two studies were at low overall risk of bias (Li 2003; Giniger 2005); two at high overall risk of bias (Turkun 2010; Aka 2017); and the remaining 67 at unclear overall risk of bias.

\section{Effects of interventions}

See: Summary of findings for the main comparison CP gel in tray versus placebo for whitening teeth; Summary of findings $2 \mathrm{HP}$ gel in tray versus placebo for whitening teeth; Summary of findings 3 HP strips versus placebo for whitening teeth; Summary of findings $4 \mathrm{CP}$ paint-on gel versus placebo for whitening teeth; Summary of findings 5 HP paint-on gel versus placebo for whitening teeth; Summary of findings 6 SHMP chewing gum versus placebo for whitening teeth; Summary of findings 7 STPP chewing gum versus placebo for whitening teeth; Summary of findings 8 HP mouthwash versus placebo for whitening teeth; Summary of findings $9 \mathrm{CP}$ gel in tray versus $\mathrm{CP}$ gel in tray for whitening teeth; Summary of findings $10 \mathrm{CP}$ gel in tray versus HP gel in tray for whitening teeth; Summary of findings 11 HP strips versus CP gel in tray for whitening teeth; Summary of findings 12 HP strips versus HP gel in tray for whitening teeth; Summary of findings 13 HP strips versus HP strips (different concentrations) for whitening teeth; Summary of findings 14 CP paint-on gel versus HP strips for whitening teeth; Summary of findings 15 HP paint-on gel versus HP strips for whitening teeth; Summary of findings 16 CP painton versus $\mathrm{CP}$ paint-on (different concentrations) for whitening teeth; Summary of findings $17 \mathrm{CP}$ paint-on versus HP paint-on for whitening teeth; Summary of findings $18 \mathrm{HP}$ paint-on versus HP paint-on for whitening teeth; Summary of findings 19 SPC painton versus $\mathrm{CP}$ paint-on for whitening teeth

In our review, we tried to do the analyses based on duration as early effects, intermediate effects and long-term effects. However due to high heterogeneity, many trials could not be combined in the individual categories. Also not many trials reported long-term effects of bleaching agents. We also felt that combining varying methods of application and concentrations would not give a clearer picture to the customers who would want to choose among the different products available over-the-counter. With these considerations in mind, we chose to conduct the analyses based on the concentration of active agents and method of application. Only those trials having all the variables (concentration, active agent, method and timing of application and duration) similar were combined in meta-analyses. The rest of the comparisons were reported independently.

As most of the trials reported data at multiple time points, the longest follow-up time was considered in the meta-analysis as described by the Cochrane Handbook for Systematic Reviews of Interventions section 9.3.4 (Higgins 2011). However, the other time points have also been discussed descriptively wherever relevant, as bleaching effect at the shorter time duration may have clinical implications. We discussed all the outcomes separately in the individual comparisons for ease of understanding.

\section{Bleaching agent versus placebo}

\section{1a. CP gel in tray versus placebo}

\section{Tooth whitening - assessed by the dentist}

Six trials studied carbamide peroxide (CP) versus placebo (Russell 1996; Matis 1998; Browning 2008; Mederios 2008; Hyland 2015; Aka 2017) with Russell 1996 and Matis 1998 expressing dichotomous data. Browning 2008; Hyland 2015; and Aka 2017 were the multiarm trials from which we analysed data comparing CP gel in tray to placebo. Mederios 2008 was described qualitatively and not included in meta-analyses as the data were represented as median scores.

\section{Continuous outcome}

Hyland 2015 and Aka 2017 used the digital images with CIEL*a* ${ }^{\star}$ scoring while Browning 2008 and Mederios 2008 used the Vita shade guide. Aka 2017 analysed the groups based on baseline tooth shade as light shade, medium dark and dark shades. In all categories, the test group showed favourable results for whitening of teeth compared to placebo gel.

Hyland 2015 compared 5\% CP formulation containing sodium tripolyphosphate for 2 weeks and showed $L^{*}$ values significantly higher compared to placebo group (mean difference (MD) 4.56, 95\% confidence interval (CI) 1.52 to $7.59 ; 1$ trial, 21 participants; Analysis 1.1).

Browning 2008 used $10 \%$ CP with a desensitiser versus placebo gel for 2 weeks with the test group showing better Vita shade scores for lightness compared to placebo (MD 4.70,95\% $\mathrm{Cl} 3.28$ to $6.12 ; 1$ trial, 37 participants; Analysis 1.1).

Irrespective of the original shade, the CP group showed significantly higher E values compared to placebo in Aka 2017 (light shade: MD 4.50, 95\% Cl 4.04 to 4.96; 1 trial, 179 teeth; medium dark 
shade: MD 6.90, 95\% Cl 6.35 to $7.45 ; 1$ trial, 172 teeth; darker shade: MD 10, 95\% Cl 9.44 to $10.56 ; 1$ trial, 176 teeth) (Analysis 1.1).

Mederios 2008 compared $10 \% \mathrm{CP}$ to placebo for 21 days and presented data as a medium score and interquartile range. The median increase in lightness of the teeth in the test group was 3 units based on the value-ordered Vitapan shade guide. This improvement in lightness was maintained for 6 months in $88 \%$ of this group. In the placebo group, $8 \%$ has a 2 -unit reduction in tooth colour at day 21 .

\section{Dichotomous outcome}

Russell 1996 and Matis 1998 studied the effect of 10\% CP and the data at 2 weeks and 6 months for these trials were combined in the meta-analysis. Russell 1996 used the Vita shade guide an categorised the scale for measurement as darker, same or lighter. The lighter shade score was considered as events. Matis 1998 used an ordinal scale where 0 represented no change; 1 represented slight change; 2 was moderate change and 3 represented a large colour change. Scores 2 and 3 were combined and considered as events.

A significant lightening effect was shown at 2 weeks. In addition, the lightening effect lasted when tested at 6 months for the majority of the subjects (risk ratio (RR) $6.74,95 \% \mathrm{Cl} 3.15$ to 14.40 ; 2 trials, 109 participants; Analysis 2.1). Russell 1996 showed similar results at 1 week, 6 weeks and 3 months interval. Matis 1998 also showed similar results at 4 weeks.

\section{Tooth whitening - reported by the patient}

Mederios 2008 recorded volunteers satisfaction by administering a questionnaire at the end of 21 days of treatment and Aka 2017 reported patient satisfaction after 10 days, 14 days, 2 weeks and 6 months of bleaching, which was self-assessed on a 7-point scale, with 1 correlating to no satisfaction and 7 to maximum satisfaction. Patients in the CP group were more satisfied by the bleaching effect in both the trials.

\section{Adverse effects}

Most common adverse effects were gingival sensitivity, tooth sensitivity, gastrointestinal sensitivity in both the groups although they were transient and mild (Matis 1998). Sensitivity to hot and cold, gingival sensitivity, tongue and throat sensitivity was reported by Myers 2003 which was more for the test group. Mederios 2008 also reported tooth sensitivity, which was more for the CP group compared to the placebo group.

Other trials did not report any adverse reactions. No other secondary outcomes were reported.

\section{1b. HP gel in tray versus placebo}

Tooth whitening - assessed by the dentist

Two trials studied the effect of hydrogen peroxide (HP) gel versus placebo (Myers 2003; Mohan 2008). Mohan 2008 reported significantly greater $L^{*}$ values at 3, 7 and 14 days in the $6 \% \mathrm{HP}$ gel group indicating greater lightness (14 days: MD 3.08, 95\% Cl 2.28 to 3.88; 1 trial, 49 participants; Analysis 3.1).

Myers 2003 used Vita shade guide and found the 3\% HP group with significantly lighter shades at 2, 12 and 26 weeks compared to the placebo group. Mean shade change was 4.2 Vita shade tabs at 2 weeks. At 26 weeks ( 6 months), the degree of whitening was 4.1 tabs.

No other outcomes were reported.

\section{1c. HP strips versus placebo}

See Figure 3. 
Figure 3. Funnel plot of comparison: 4 HP strip versus placebo, outcome: 4.1 Tooth whitening - assessed by the dentist.

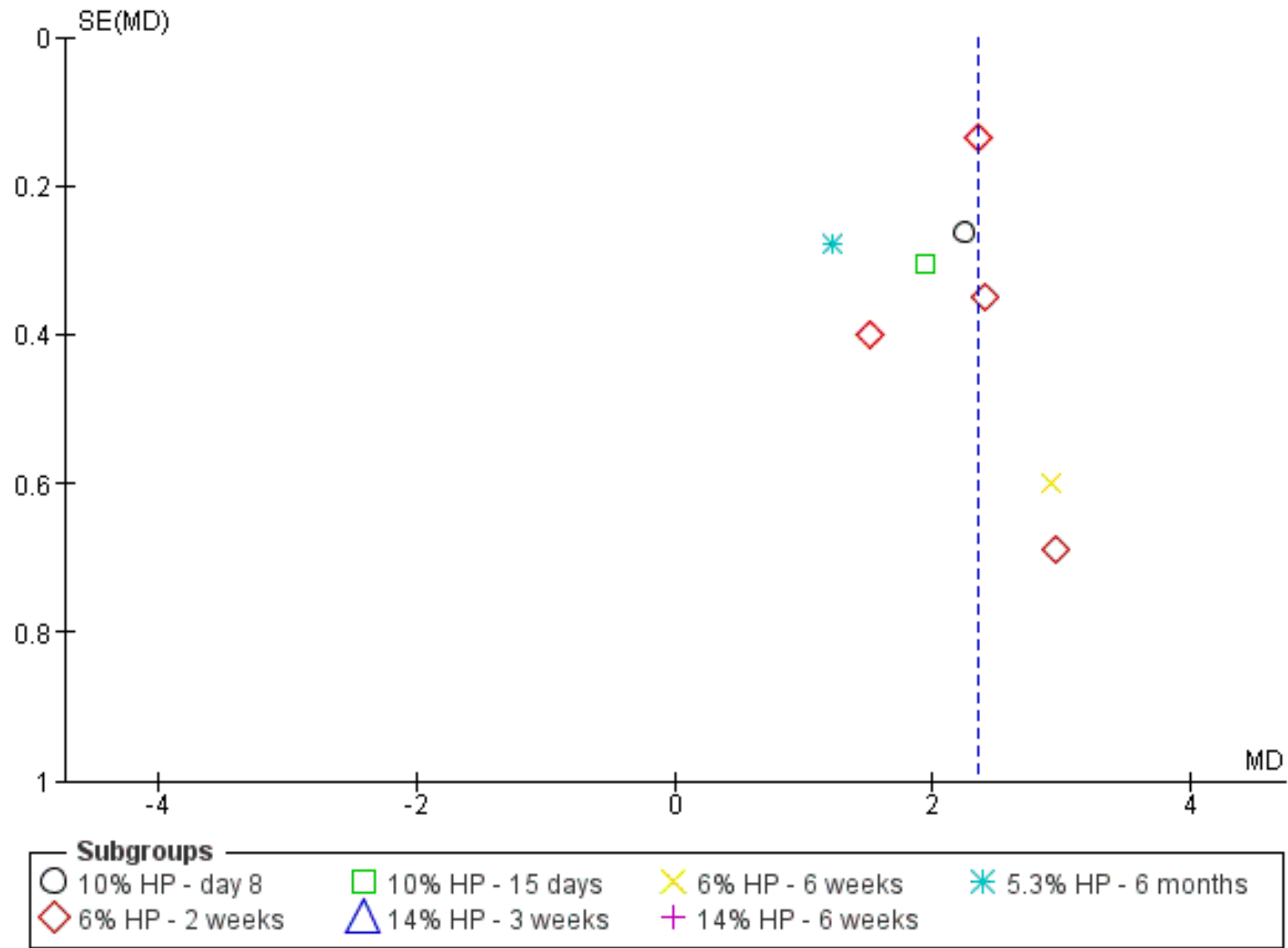

\section{Tooth whitening - assessed by the dentist}

Ten trials studied the effect of HP strips against placebo (Kugel 2000; Gerlach 2002; Garcia-Godoy 2004; Gerlach 2004e; Swift 2004; Wong 2004; Bizhang 2007; Papas 2009; Swift 2009; Bruhn 2012).

Gerlach 2004e who studied $10 \%$ HP for 8 days against a placebo showed greater $L^{*}$ value for the test group indicating better lightness (MD 2.24, 95\% Cl 1.72 to 2.76; 1 trial, 36 participants; Analysis 4.1).

The Kugel 2000; Wong 2004; Bizhang 2007; Swift 2009 trials were combined to analyse the effect of $6 \% / 5.3 \% \mathrm{HP}$ versus placebo for 2 weeks. $L^{*}$ values significantly higher in the HP group indicated greater lightness compared to placebo (MD 2.24, 95\% Cl 1.83 to 2.66; 4 trials, 195 participants; Analysis 4.1).

Papas 2009 showed similar results as Gerlach 2004e at 15 days (MD $1.93,95 \% \mathrm{Cl} 1.34$ to $2.52 ; 1$ trial, 40 participants; Analysis 4.1).

Swift 2004 and Garcia-Godoy 2004 studied 14\% HP versus placebo for 3 weeks and 6 weeks, using Vita shade guide and CIEL*a* $b^{\star}$ scoring respectively. The $14 \%$ HP group demonstrated improvement in shades relative to the placebo strip group with a difference between treatment groups favouring the peroxide group in both trials (Swift 2004: MD 7.60, 95\% Cl 6.18 to 9.02; 1 trial, 28 participants; Garcia-Godoy 2004: MD 5.16, 95\% Cl 4.21 to $6.11 ; 1$ trial, 35 participants (Analysis 4.1)).

Swift 2009 reported the HP group with better lightening effect compared to placebo at 4 weeks and 6 weeks ( 6 weeks: MD 2.90, $95 \% \mathrm{Cl} 1.73$ to $4.07 ; 1$ trial, 37 participants; Analysis 4.1).

5.3\% HP versus placebo studied by Gerlach 2002e showed that most of the initial colour change remained at 6 months post-treatment with the whitening strip group continuing to demonstrate highly significant improvements in tooth colour relative to the placebo group (MD 1.21, 95\% $\mathrm{Cl} 0.67$ to $1.75 ; 1$ trial, 52 participants; Analysis 4.1).

\section{Tooth whitening - reported by the patient}

One trial (Bruhn 2012) compared 14\% HP against placebo and reported the tooth colour satisfaction scale (TCSS). One trial (Wong 2004) reported patient satisfaction, rated using a product satisfaction questionnaire. Satisfaction regarding the whitening effect of the product was highest for the strip group compared to the placebo group in both trials.

\section{Adverse effects}

Treatment with HP whitening strips was generally well tolerated with adverse events confined to symptoms only. Mild and transient 
tooth sensitivity and oral irritation were the most common adverse events reported in most of the trials (Kugel 2000; Gerlach 2002e; Garcia-Godoy 2004; Gerlach 2004e; Swift 2004; Papas 2009; Swift 2009).

\section{Oral health-related quality of life}

One trial (Bruhn 2012) compared 14\% HP strips against placebo and reported the oral health-related quality of life (OHRQoL). Wong 2004 reported the standardised response mean for the oral health impact profile (OHIP) and its domains for subjects in each category of the global transition judgement on whether they were dissatisfied (-1), neutral (0) or satisfied (1). No significant impact of tooth whitening was found in both trials on OHRQoL.

No trial reported on patient's level of comfort with treatment.

\section{1d. CP paint-on gel versus placebo}

\section{Tooth whitening - assessed by the dentist}

Nathoo 2002 compared 18\% CP gel to placebo using Vita shade guide and the mean changes from baseline tooth shade rank score were compared across treatment groups. CP paint-on gel exhibited a mean change score of 2.54 units greater that placebo at 2 weeks interval and 3.5 units higher at 3 weeks interval ( 3 weeks: MD 3.50, $95 \% \mathrm{Cl} 3.12$ to $3.88 ; 1$ trial, 77 participants; Analysis 5.1).

\section{Patient comfort}

In Nathoo 2002 participants anecdotally reported that the products were extremely easy to apply and did not interfere with speech or life style.

No other outcomes were reported.

\section{1e. HP paint-on gel versus placebo}

\section{Tooth whitening - assessed by the dentist}

Collins 2004a used Vita shade and Xu 2007 used digital image analysis with $\mathrm{CIEL}^{*} \mathrm{a}^{*} \mathrm{~b}^{\star}$ scoring to test $6 \% \mathrm{HP}$ paint-on gel versus placebo. We combined the 2-week data using standardised mean difference (SMD) and the paint-on group exhibited greater whitening compared to placebo (SMD $0.67,95 \% \mathrm{Cl} 0.19$ to $1.14 ; 2$ trials, 148 participants; Analysis 6.1). Similar results were found by Collins 2004a at 1 week interval.

\section{Adverse effects}

Collins 2004a reported the HP group participants with more gum irritation (reddening) compared to placebo although the symptoms were mild. One case of tooth sensitivity was noted.

No other outcomes were reported.

\section{1f. SHMP chewing gum versus placebo}

\section{Tooth whitening - assessed by the dentist}

Among the three trials comparing sodium hexametaphosphate (SHMP) chewing gum to placebo, Biesbrock 2004 used digital image analysis with $\mathrm{CIEL}^{\star} \mathrm{a}^{*} \mathrm{~b}^{\star}$ scoring using 7.5\% SHMP; Walters 2004 and Porciani 2006 used the Lobene stain index with 5.65\% and $4 \%$ SHMP respectively. Accumulation of stain in all the trials was lower in the test group compared to placebo in Biesbrock 2004 at 1 day and 2 days ( 2 days: MD $0.89,95 \% \mathrm{Cl} 0.77$ to $1.01 ; 1$ trial, 37 participants; Analysis 7.1); Walters 2004 at 3 days (MD 2.60, 95\% Cl 1.45 to $3.75 ; 1$ trial, 20 participants; Analysis 7.1 ) also found the test group exhibiting lesser stains compared to placebo.

Porciani 2006 reported 33\% reduction in induced stain formation by the test group compared to placebo at 12 weeks (MD - $0.14,95 \%$ $\mathrm{Cl}-0.38$ to 0.10 ; 1 trial, 108 participants; Analysis 7.1).

No other outcomes were reported.

\section{1g. STPP chewing gum versus placebo}

\section{Tooth whitening - assessed by the dentist}

Porciani 2010 studied 1\% sodium tripolyphosphate (STPP) against a placebo using Lobene composite stain index which was reduced by $8.8 \%$ in the test group and increased by $8 \%$ in the control group (MD $0.18,95 \% \mathrm{Cl} 0.10$ to $0.26 ; 1$ trial, 108 participants; Analysis 8.1 ).

No other outcomes were reported.

\section{1h. HP mouthwash versus placebo}

\section{Tooth whitening - assessed by the dentist}

Hasturk 2004 evaluated the tooth whitening effect of 1.5\% HP fluoridated rinse by dichotomising the change in shades as measured by the Lobene stain index. Data were expressed in odds ratios (OR) at 1 month, 3 months, 6 months and overall tooth whitening. Generic inverse variance method was used and log of odds ratio and standard error were calculated for the analysis.

Compared with placebo, the HP mouthrinse group was more than 7 times as likely to show whitening at 1 month and 3 months. Greater whitening was also demonstrated by the mouthwash group at 6 months (OR $10.89,95 \% \mathrm{Cl} 5.08$ to 23.35; 1 trial, 78 participants; Analysis 9.1). Overall tooth whitening was almost 8.7 times more likely among the mouthwash group than the placebo group.

No other outcomes were reported.

\section{Bleaching agent versus bleaching agent}

\section{2a. $C P$ tray versus $C P$ tray}

See Figure 4. 
Figure 4. Funnel plot of comparison: $11 \mathrm{CP}$ tray versus $\mathrm{CP}$ tray, outcome: 11.1 Tooth whitening - assessed by the dentist.

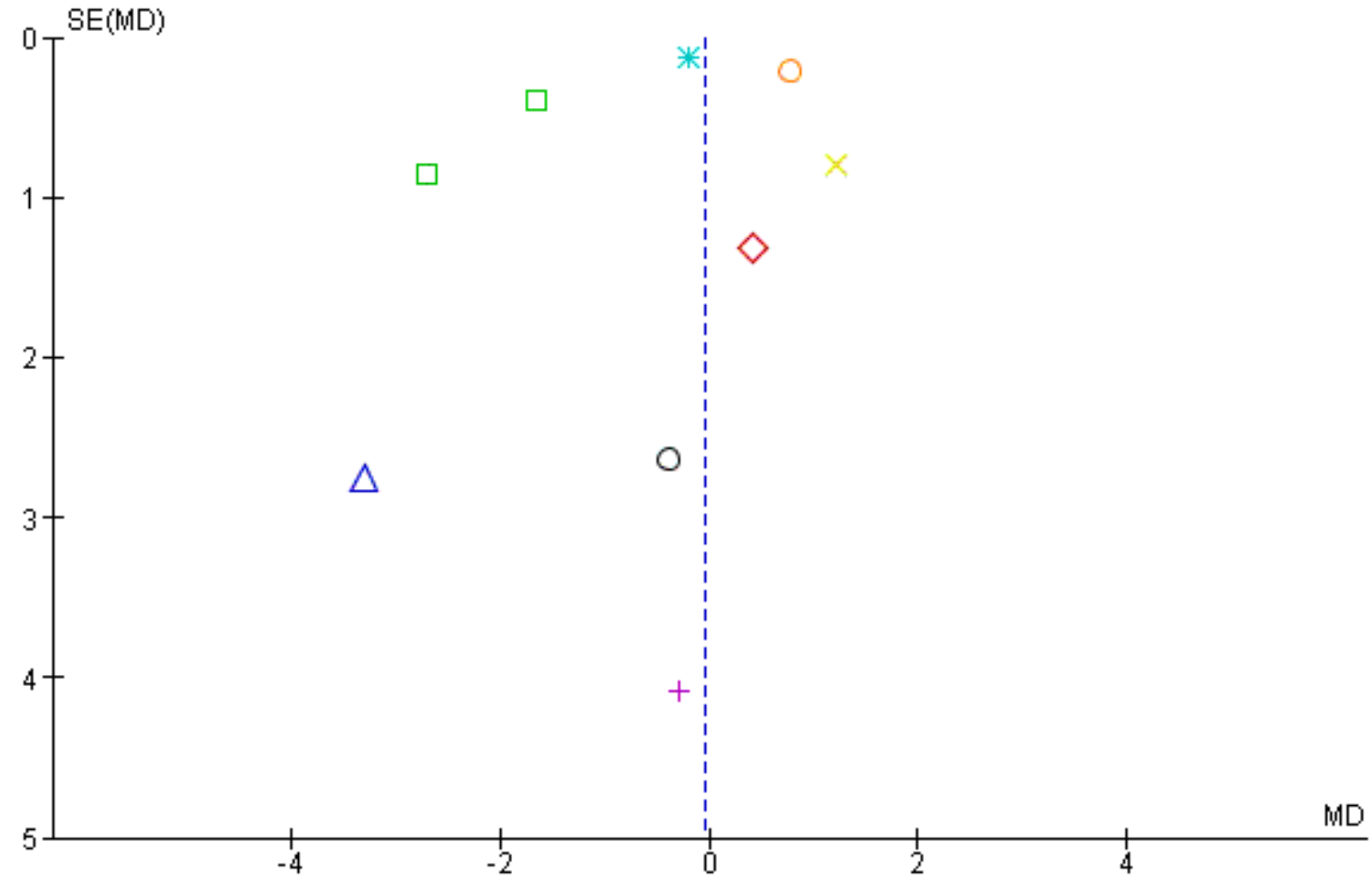

Subgroups

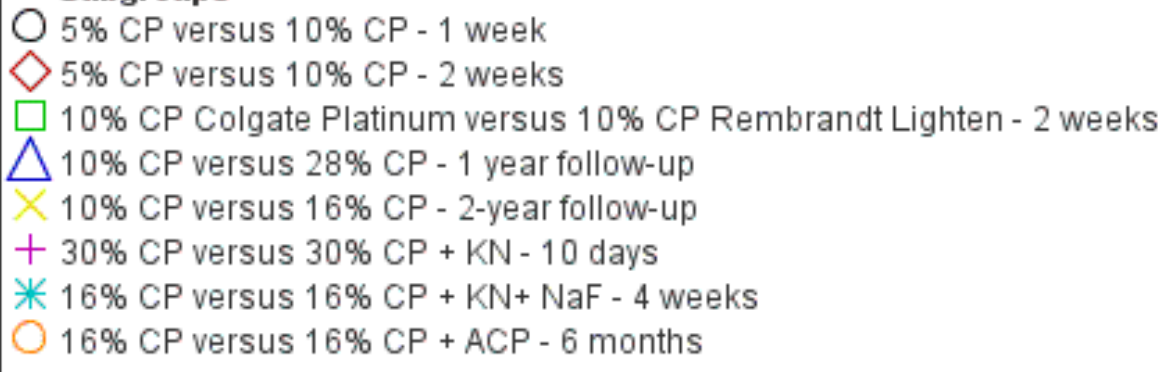

Among the 18 trials, six used value-oriented/Vita classical shade guide for measuring colour change (Cibirka 1999; Kihn 2000; Giniger 2005; Browning 2008; Gallo 2009; Kose 2011).

Seven trials used electronic instruments for colour change analysis (spectrophotometer, colorimeter, digital analysis) with $\mathrm{CIEL}^{\star} \mathrm{a}^{*} \mathrm{~b}^{\star}$ scoring system (Kowitz 1994; Matis 2000; Tsubura 2005; Matis 2006; Turkun 2010; Navarra 2014; Hyland 2015).

Three trials (Mokhlis 2000; Nathoo 2001; Meireles 2010) measured whitening using a combination of shade guide and electronic instruments. All these trials reported data in CIEL $a^{*} b^{\star}$ scoring which was considered for analysis.

Krause 2008 reported patient contentment using inter-personal modal intensity comparison where the patient pressed the bulb of a manometer in proportion to their objective concerning the bleaching outcome.
Meireles 2010 in one of his follow-up reports used oral impact on daily performance (OHIP) scoring to assess oral health-related quality of life.

Tam 2001 used photographic evaluation and visual analogue scale (VAS), which the patients marked daily in the evening before the next day's bleaching treatment.

\section{Tooth whitening - assessed by the dentist}

\section{Dichotomous outcome}

Data were presented as an ordinal scale of darker, same or lighter in Cibirka 1999 trial. Number of participants in the lighter shade category were considered as events. A significant degree of lightening of tooth shade relative to baseline values at 2 weeks was demonstrated for both Opalescence and NiteWhite Excel ( $10 \%$ $\mathrm{CP}$ formulations), however no evidence of a difference was found 
between the groups (RR $1.03,95 \% \mathrm{Cl} 0.90$ to $1.18 ; 1$ trial, 66 participants; Analysis 10.1).

\section{Continuous outcome}

Nathoo 2001 which compared 5\% and 10\% CP found no evidence of a difference between the groups. Mean unit change after 7 days for $5 \%$ group was $6.7 \pm 1.9$ while for the $10 \%$ group was $5.7 \pm 2$ though the result was not statistically significant (MD $-0.38,95 \% \mathrm{Cl}-5.55$ to 4.79; 1 trial, 58 participants; Analysis 11.1).

Hyland 2015 a multiarm trial compared $5 \%$ and $10 \%$ CP with STPP formulations. It found no evidence of a difference between $55 \%$ and $10 \% \mathrm{CP}$ in tooth whitening at 2 weeks following daily wear of tooth whitening trays for 2 hours a day (MD $0.41,95 \% \mathrm{Cl}-2.17$ to $2.98 ; 1$ trial, 21 participants; Analysis 11.1).

Colgate Platinum (CLP) and Rembrandt Lighten (RL) (both 10\% CP formulations) compared in Kowitz 1994; Nathoo 1994, showed significantly higher $L^{*}$ values for the CLP group compared to the RL group (MD -1.92, 95\% Cl -2.80 to -1.03; 2 trials, 88 participants; Analysis 11.1).

Both daytime at-home bleaching system including 28\% CP gel with a non-custom tray (Meta tray) and conventional overnight athome 10\% CP gel with a custom tray (Opalescence PF) in Turkun 2010 produced whitening compared to the baseline. Intergroup comparison showed $10 \% \mathrm{CP}$ overnight bleaching group to be superior to the day time 20 -minute bleaching at 2 weeks and 1 year follow-up (1 year follow-up: MD -3.30, 95\% Cl -8.71 to 2.11 ; 1 trial, 20 participants; Analysis 11.1).

Follow-up at 2 years (Meireles 2010) (MD 1.20, 95\% Cl -0.35 to 2.75; 1 trial, 81 participants; Analysis 11.1) revealed that the tooth shade remained significantly lighter compared to baseline in both the groups comparing $10 \%$ and $16 \%$ CP. However, tooth shade relapse showed no difference between the groups.

Gallo 2009 who used different concentrations of CP with and without desensitisers, showed no significant difference between the groups in terms of colour change at 10 days (MD $-0.30,95 \% \mathrm{Cl}$ -8.28 to $7.68 ; 1$ trial, 40 participants; Analysis 11.1). Similar results were observed in Kose 2011 at 4 weeks (MD $-0.20,95 \% \mathrm{Cl}-0.44$ to 0.04; 1 trial, 60 participants; Analysis 11.1).

At 180 days the amorphous calcium phosphate (ACP) group retained nearly $10 \%$ more of their original whitening treatment result compared to control in Giniger 2005 (MD 0.78, 95\% Cl 0.37 to 1.19; 1 trial, 27 participants; Analysis 11.1).

Polanight (PN) and Opalescence (OP) (both 10\% CP formulations) compared in Tsubura 2005, showed significant difference in $L^{\star}$ values for PN compared to OP. Bleaching with PN was considered more effective than that with $O P$ in young patient group and in women (MD 1.46, 95\% Cl 0.13 to 2.79; 1 trial, 116 participants; Analysis 11.2).

Five-year data of $10 \%$ CP versus $15 \%$ CP was analysed from Matis 2006. No evidence of a difference was found between the groups in relation to shade change (MD $-1.47,95 \% \mathrm{Cl}-3.56$ to $0.62 ; 1$ trial, 58 participants; Analysis 11.2).

Kihn 2000 showed the 15\% CP group to have a larger amount of shade change than did the control group (MD 1.65, 95\% Cl 0.22 to
3.08; 1 trial, 52 participants; Analysis 12.1). Matis 2000 also showed similar results (MD 2.22, 95\% Cl 1.29 to 3.15; 1 trial, 25 participants; Analysis 12.2).

Data for $10 \%$ CP with and without potassium nitrate and sodium fluoride used in two trials (Browning 2008; Navarra 2014), was combined in the meta-analysis and showed no evidence of a difference between the whitening systems (standardised mean difference (SMD) $0.32,95 \% \mathrm{Cl}-0.20$ to $0.84 ; 2$ trials, 58 participants; Analysis 12.3).

\section{Tooth whitening - reported by the patient}

Krause 2008 reported patient contentment with the bleaching outcome with an inter-modal intensity comparison. Although the patient contentment score in the $17 \%$ group was higher, no evidence of a difference was found between both groups at 2 weeks and 3 weeks interval ( 3 weeks: MD 2.60, 95\% Cl 2.57 to 2.63; 1 trial, 20 participants; Analysis 11.3). All participants completing the 2-year evaluation in Meireles 2010 reported no evidence of a difference between treatment groups regarding the patient-reported satisfaction in relation to whitening and retention of whitening. Meireles used a questionnaire to rate patient satisfaction regarding whitening outcome in one of his follow-up reports. No evidence of a difference in the whitening effect was found as reported by patients in both CP $10 \%$ and CP $16 \%$ groups.

In Matis 2006, at the 5th year evaluation appointment, seven were very pleased with how their teeth look at that time in both $10 \%$ and $15 \%$ groups. 14 of them were very pleased and seven reported they were not pleased with the appearance in both groups.

Tam 2001 presented data in range using the VAS scale reported by the patients. We did not use these values for analysis according to the Cochrane Handbook for Systematic Reviews of Interventions section 7.7.3.6 (Higgins 2011). The authors reported the lack of evidence of a difference in perceived whiteness between the groups with and without the desensitiser.

\section{Patient comfort}

One of Meireles 2010 follow-up reports used a questionnaire to rate patient comfort on 1 to 5 scale (1 representing agree and 5 representing disagree). Participants from both whitening regimens reported positive opinions about the treatment. Despite this, the CP 10\% group reported less interference with the tray when talking $(P=0.02)$ and less discomfort after application $(P=0.04)$ compared to the CP $16 \%$ group.

\section{Adverse effects}

Higher concentrations of $\mathrm{CP}$ had more sensitivity compared to lower concentrations (Nathoo 2001; Matis 2006; Krause 2008; Meireles 2010). Navarra 2014 and Browning 2008 reported bleaching agents with desensitiser with significantly lower sensitivity than the bleaching product that did not contain desensitising agents. Kihn 2000; Matis 2000; Gallo 2009 did not report any significant difference in the tooth and gingival irritation between the two groups. Tam 2001; Giniger 2005; Tsubura 2005; Kose 2011 reported mild irritation and sensitivity in both groups. Kowitz 1994; Nathoo 1994; Cibirka 1999; Turkun 2010; Hyland 2015 did not report any adverse events. 


\section{Oral health-related quality of life}

In Meireles 2010 2-year follow-up report the 16\% CP group showed greater bleaching effect than $10 \%$ CP, however, OHRQoL was similar for both groups.

\section{2b. CP tray versus HP tray}

See Figure 5.

\section{Figure 5. Forest plot of comparison: 13 CP tray versus HP tray, outcome: 13.1 Tooth whitening - assessed by the} dentist.

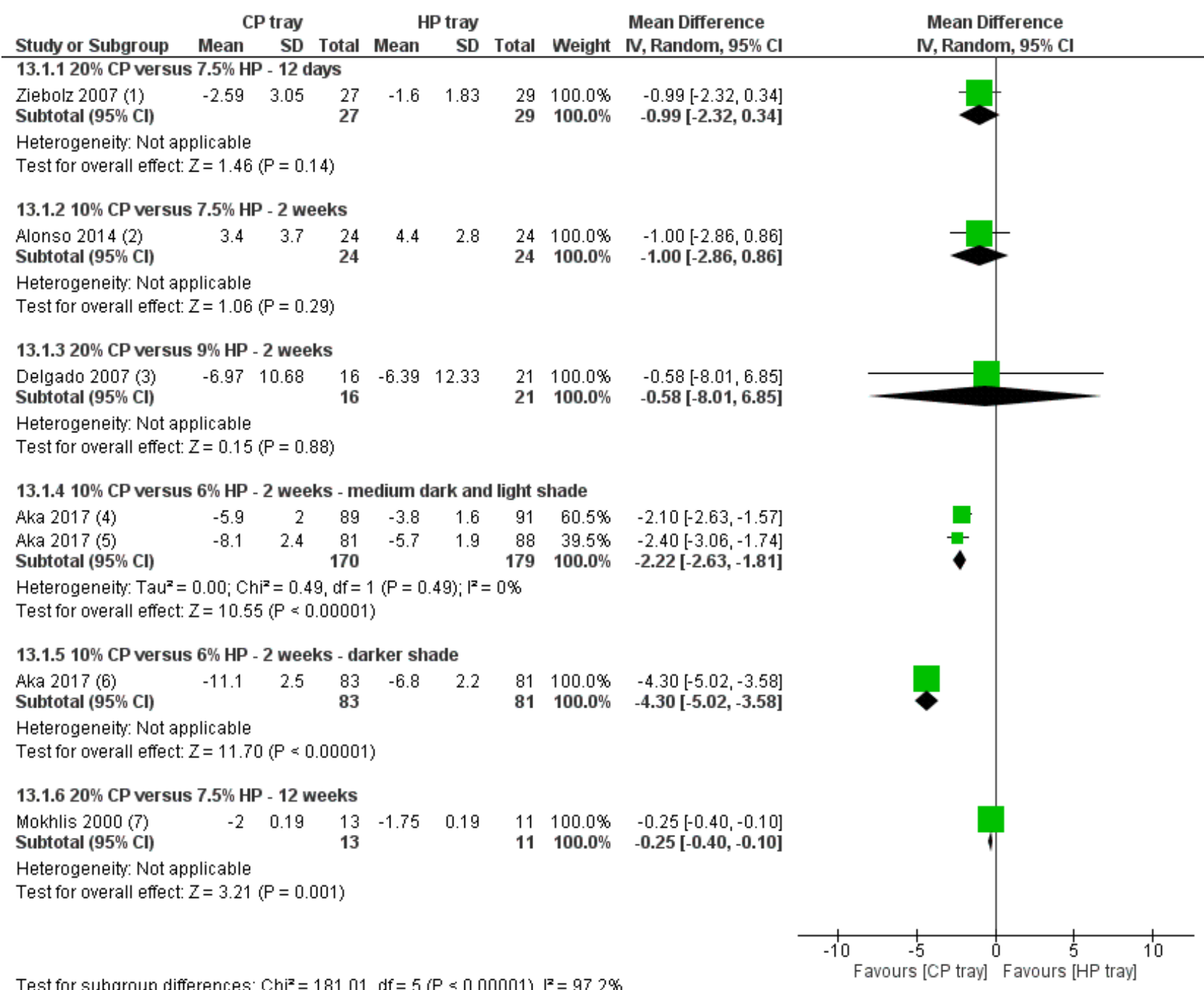

\section{Footnotes}

(1) Group 1:20\% CP; Group 2:7.5\% HP. CIELAB scoring was used. Greater value indicates better lightness.

(2) Group 1: $7.5 \% \mathrm{HP}$; Group 2: 10\% CP. Vita shade guide was used.Greater value indicates better lightness.

(3) Group 1: 20\% CP; Group 2: $9 \%$ HP. Vita shade guide was used.Greater value indicates better lightness.

(4) (Tooth level analysis) Group 1:10\% CP; Group 2: 6\% HP for lighter teeth. CIELAB scoring used.Greater value indicates better lightness.

(5) (Tooth level analysis) Group 1: $10 \% \mathrm{CP}$; Group 2: $6 \% \mathrm{HP}$ for medium dark teeth after 14 days of bleaching. CIELAB scoring was used.

(6) (Tooth level analysis) Group 1:10\% CP; Group 2: 6\% HP for darker teeth after 14 days of bleaching. CIELAB scoring was used.

(7) Group 1: 20\% CP; Group 2: 7.5\% HP. CIELAB scoring was used.Greater value indicates better lightness.

\section{Tooth whitening - assessed by the dentist}

Among the seven trials, three used value-oriented/Vita classical shade guide for measuring colour change (Alonso 2006; BergaCaballero 2006; Delgado 2007). Two trials used electronic instruments for colour change analysis (spectrophotometer, colorimeter, digital analysis) with CIEL*a*b* scoring system (Ziebolz 2007; Alonso 2014). Two trials (Mokhlis 2000; Aka 2017) measured whitening using a combination of shade guide and electronic instruments. All these trials reported data in $\mathrm{CIEL}^{\star} \mathrm{a}^{\star} \mathrm{b}^{\star}$ scoring which was considered for analysis.

Both $7.5 \%$ HP and 20\% CP in Ziebolz 2007 resulted in significant colour improvements in all parameters compared to baseline. Though the reduction in yellowness was significantly more in the $20 \%$ CP group compared to $7.5 \% \mathrm{HP}$, improvement in lightness $\left(\mathrm{L}^{*}\right)$ 
showed no evidence of a difference over the 12 days period (MD $-0.99,95 \% \mathrm{Cl}-2.32$ to $0.34 ; 1$ trial, 56 participants; Analysis 13.1 ).

We compared $10 \% \mathrm{CP}$ to $7.5 \% \mathrm{HP}$ used in a multiarm trial (Alonso 2014). There was no evidence of a difference between the groups though there was an increase in lightness compared to baseline in both groups (MD $-1,95 \% \mathrm{Cl}-2.86$ to $0.86 ; 1$ trial, 48 participants; Analysis 13.1).

Delgado 2007 9\% HP group showed a statistically significant 1.54 greater shade rank score unit reduction in the mean tooth shade rank score after 5 days. However, after 7 and 14 days the rank greater score unit reductions were 1.18 and 0.83 for the $9 \%$ HP group compared to the $20 \% \mathrm{CP}$ group, but not statistically significant (MD $-0.58,95 \% \mathrm{Cl}-8.01$ to $6.85 ; 1$ trial, 37 participants; Analysis 13.1).

Irrespective of the original shade, 10\% CP bleaching groups in Aka 2017 multiarm trial showed significantly higher E values compared to $6 \% \mathrm{HP}$ : medium dark and light shade: MD $-2.22,95 \% \mathrm{Cl}-2.63$ to -1.81 ; 2 trials, 349 teeth; darker shade: MD $-4.30,95 \% \mathrm{Cl}-5.02$ to -3.58; 1 trial, 164 teeth (Analysis 13.1).

Similar concentrations of CP and HP were used in another trial (Mokhlis 2000) which favoured the 20\% CP group for better lightness in the first 14 days. But at the end of the study there was no evidence of a difference between the two groups (12 weeks: MD $0.25,95 \% \mathrm{Cl} 0.10$ to 0.40 ; 1 trial, 24 participants; Analysis 13.2).

Berga-Caballero 2006 found that the changes in colour ranged from 1 to 10 shades of the Vita shade guide's brightness-based classification; the whitening success percentage was between
$315 \%$ and $100 \%$ on the Jane-Roig scale, which is based on the greatest percentage of whitening that can be achieved in a tooth, depending on its initial colour. Both $10 \% \mathrm{CP}$ applied for varying times and 3.5\% HP were effective.

Alonso 2006 reported no evidence of a difference between the groups using 3.5\% HP along with a desensitiser (potassium nitrate) versus $10 \% \mathrm{CP}$.

\section{Tooth whitening - reported by the patient}

Aka 2017 reported that the 10\% CP/PF (Opalescence PF) groups were more satisfied with the bleaching effect than those in the $6 \%$ HP groups.

\section{Patient comfort}

In Ziebolz 2007 both $7.5 \%$ HP and 10\% CP groups showed a similar proportion of subjects with complaints regarding comfort of the bleaching treatment with no significant differences on a 4-point ordinal scale.

\section{Adverse effects}

Mokhlis 2000; Ziebolz 2007; Aka 2017 did not report any significant difference in the tooth and gingival irritation between the two groups. Alonso 2006; Berga-Caballero 2006; Delgado 2007; Alonso 2014 reported mild irritation and sensitivity in both groups.

No other outcomes were reported.

\section{2c. HP strips versus CP tray}

See Figure 6. 
Figure 6. Forest plot of comparison: 14 HP strip versus CP tray, outcome: 14.1 Tooth whitening - assessed by the dentist.

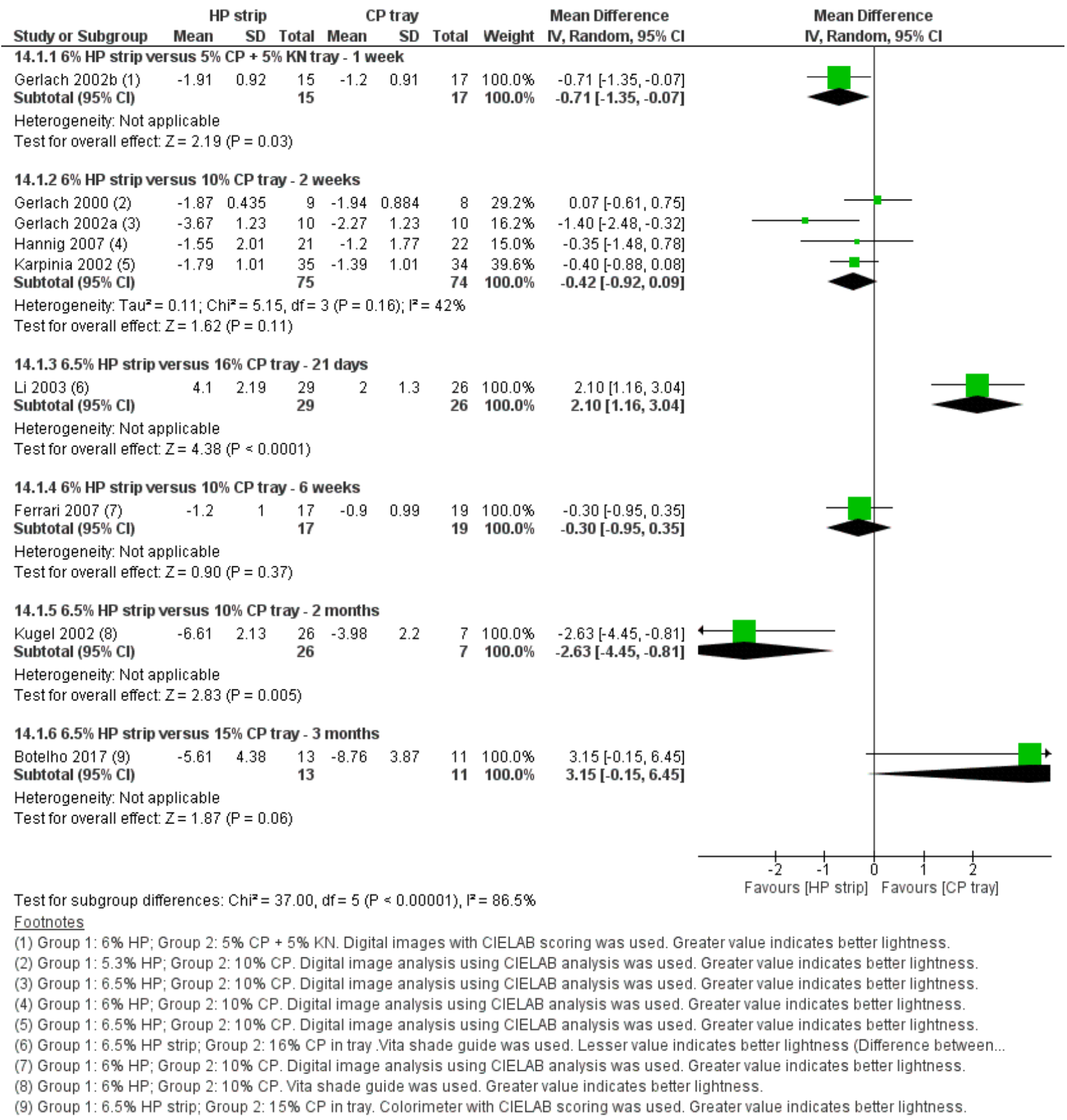

\section{Tooth whitening - assessed by the dentist}

Among the 10 trials included in this group, seven trials used digital image analysis with $\mathrm{CIEL}^{*} \mathrm{a}^{\star} \mathrm{b}^{\star}$ scoring (Gerlach 2000; Gerlach 2002b; Gerlach 2002a; Karpinia 2002; Ferrari 2007; Hannig 2007; Costa 2012). One trial (Botelho 2017) used a colorimeter with $\mathrm{CIEL}^{\star} \mathrm{a}^{\star} \mathrm{b}^{\star}$ scoring and two trials used the Vita shade guide (Kugel 2002; Li 2003).

Gerlach 2002b comparing 6\% HP strips to 5\% CP with desensitiser for 1 week, revealed $59 \%$ greater composite colour change for the strip group compared to the tray (MD $-0.71,95 \% \mathrm{Cl}-1.35$ to $-0.07 ; 1$ trial, 32 participants; Analysis 14.1).

Gerlach 2000; Gerlach 2002a; Karpinia 2002; and Hannig 2007 compared similar concentrations of HP strips and 10\% CP in tray and were combined in a meta-analysis. The combined analysis favoured the HP strip group in comparison to $10 \%$ CP in tray (MD $-0.42,95 \% \mathrm{Cl}-0.92$ to $0.09 ; 4$ trials, 149 participants; Analysis 14.1). 
Li 2003 (who used 6.5\% HP strips versus 16\% CP in tray) favoured the tray group (MD 2.10, 95\% Cl 1.16 to 3.04; 1 trial, 55 participants; Analysis 14.1).

Ferrari 2007 at 6 weeks reported better whitening with HP strip group $\left(\mathrm{L}^{*}\right)$ in comparison to $10 \% \mathrm{CP}$ though the result was not statistically significant (MD $-0.30,95 \% \mathrm{Cl}-0.95$ to $0.35 ; 1$ study, 36 participants; Analysis 14.1). However, b values showed favourable results for the strip group at all intervals with statistical significance $(P=0.049)$.

Kugel 2002 reported that the 6.5\% HP strip group experienced statistically significant superior reductions in shade compared to $10 \%$ CP in tray at both 1 and 2 month time points ( 2 months: MD $-2.63,95 \% \mathrm{Cl}-4.45$ to $-0.81 ; 1$ trial, 33 participants; Analysis 14.1 ).

Intergroup comparison of $6.5 \% \mathrm{HP}$ strips versus $15 \% \mathrm{CP}$ in tray in Botelho 2017 showed that the tray group had greater overall colour changes $(E)$ than the strip group at 3 months. However, no evidence of a difference was found between the groups at 3 months (MD 3.15, $95 \% \mathrm{Cl}-0.15$ to $6.45 ; 1$ trial, 24 participants; Analysis 14.1).

Twice daily use of $14 \%$ HP or $35 \%$ CP for 2 weeks in Costa 2012 splitmouth trial resulted in significant improvement in tooth lightness relative to baseline. However, no difference was seen between the groups at 2 weeks and 1 month interval ( 1 month: MD $0.58,95 \% \mathrm{Cl}$ -0.61 to $1.77 ; 1$ trial, 24 participants; Analysis 14.2).

\section{Tooth whitening - reported by the patient}

Costa 2012 used a patient satisfaction questionnaire to assess patient satisfaction of tooth whitening. $75 \%$ of participants could not see the difference in tooth whitening between the tray and strips. 255 of the patients who noted the difference felt the side that was whitened by the tray was whiter.

Hannig 2007 reported subjective colour score as reported by the patients. No difference between the groups was noted (MD -0.41, $95 \% \mathrm{Cl}-2.05$ to 1.23 ; 1 trial, 43 participants; Analysis 14.3).

\section{Patient comfort}

Costa 2012 used the patient satisfaction questionnaire to assess patient comfort. $83 \%$ of participants in this split-mouth trial preferred the tray treatment to the strips. $75 \%$ found it more comfortable to the teeth and only $38 \%$ found it more comfortable to soft tissues. $92 \%$ of the patients reported that it was easy to do the procedure twice a day. Kugel 2002 reported that two patients in the $10 \%$ CP tray group dropped out from the study after 1 month due to inconvenient regimen.

\section{Adverse effects}

Tooth sensitivity and oral irritation were the most common adverse events. Gerlach 2005 reported sensitivity was more common with the $20 \%$ tray system. In one trial (Karpinia 2002) tooth sensitivity was more common in the strip group and oral irritation was common in the tray group. Some trials did not show any difference in adverse events between the two groups (Gerlach 2002a; Gerlach 2002b; Kugel 2002; Li 2003; Ferrari 2007; Hannig 2007; Costa 2012).

No other outcomes were reported.

\section{2d. HP strips versus HP tray}

Among the two trials in this comparison, Auschill 2012 used Vita shade guide and Gerlach 2004 used digital image analysis using CIEL*a*b* scoring.

\section{Tooth whitening - assessed by the dentist}

Gerlach 2004 which compared 14\% HP strips and 9.5\% HP in tray reported a superior 2 -fold increase in lightness in the strip group at the end of treatment ( 22 days) compared to the tray group (MD $-1.40,95 \% \mathrm{Cl}-2.35$ to $-0.45 ; 1$ trial, 29 participants; Analysis 15.1). However, initial whitening at 10 days did not differ between the groups.

Comparison of 5\% HP strips to 5.3\% HP gel in tray in Auschill 2012 showed no difference in whitening between the groups at 7 days, 2 weeks and 18 months intervals (18 months: MD $0.06,95 \% \mathrm{Cl}-2.24$ to 2.36 ; 1 trial, 28 participants; Analysis 15.1).

\section{Patient comfort}

One trial (Auschill 2012) reported patient acceptance graded based on VAS scale ranging from 0 to 10 (where $0=$ no discomfort or best acceptance and $10=$ severe discomfort or no acceptance). Statistical analysis of data demonstrated that the tray group showed statistically significantly more comfort (VAS $2.23 \pm 1.49$ ) than the strip group (VAS $3.50 \pm 1.58$ ) (MD 1.27, $95 \% \mathrm{Cl} 0.13$ to 2.41; 1 study, 28 participants; Analysis 15.2).

\section{Adverse effects}

Tooth sensitivity and oral irritation were mild and transient and did not differ between the groups (Gerlach 2004; Auschill 2012).

No other outcomes were reported.

\section{2e. HP strip versus HP strip}

\section{Tooth whitening - assessed by the dentist}

Both trials (Shahidi 2005; Oliveira 2013) used digital image analysis with $\mathrm{CIEL}^{\star} \mathrm{a}^{\star} \mathrm{b}^{\star}$ scoring.

In Oliveira 2013 the 2-hour 9.5\% HP high adhesion strips group demonstrated statistically significant lightness improvement $\left(L^{*}\right)$ than the 30 minutes 10\% HP group on 3, 5 and 9 days ( 9 days: MD $-1.50,95 \% \mathrm{Cl}-2.33$ to $-0.67 ; 1$ trial, 29 participants; Analysis 16.1 ).

Shahidi 2005 concluded that twice daily use of the very thin $10 \% \mathrm{HP}$ gel strips resulted in significant tooth whitening after 7 days. Continued use of $10 \%$ strips at 15 days yielded significant incremental whitening greater than that seen with the lower concentration (6\% HP strips) (MD 0.68, 95\% Cl 0.16 to $1.20 ; 1$ trial, 35 participants; Analysis 16.1).

\section{Adverse effects}

Oliveira 2013 reported that nearly all adverse events were classified as mild in severity. Very thin gel group exhibited lower occurrence of oral irritation and higher tooth sensitivity compared to the $6 \%$ group (Shahidi 2005).

No other outcomes were reported. 


\section{2f. HP strip versus HP mouthwash}

\section{Tooth whitening - assessed by the dentist}

Gerlach 2005 used digital image analysis with CIEL*a*b* scoring comparing HP pre-rinse to HP strips. Under the head-to-head testing conditions, 7 -day use of $10 \% \mathrm{HP}$ whitening strips resulted in significant tooth colour improvement relative to the $2 \% \mathrm{HP}$ rinse (MD $-1.10,95 \% \mathrm{Cl}-1.49$ to $-0.71 ; 1$ trial, 28 participants; Analysis 17.1).

\section{Adverse effects}

In Gerlach 2005 tooth sensitivity and oral irritation was more common in the strip group. All adverse events were mild in severity and no subjects discontinued treatment because of these events.

No other outcomes were reported.

\section{2g. CP paint-on gel versus HP strips}

\section{Tooth whitening- assessed by the dentist}

Two trials (Wong 2004; Cronin 2005) comparing 18\% CP paint-on gel with 6\% HP strips and using digital images with $\mathrm{CIEL}^{\star} \mathrm{a}^{\star} \mathrm{b}^{\star}$ scoring were combined in a meta-analysis. In both trials 6\% HP strips were more effective in tooth whitening compared to the CP paint-on gel (SMD 1.50, 95\% Cl 1.06 to 1.94; 2 trials, 102 participants; Analysis 18.1).

\section{Tooth whitening - reported by the patient}

One trial (Wong 2004) reported patient satisfaction, rated using a product satisfaction questionnaire. Satisfaction regarding the whitening effect of the product was highest for the strip group (91\%) compared to the paint-on group (33\%).

\section{Oral health-related quality of life}

Wong 2004 reported the standardised response mean for the OHIP and its domains for subjects in each category of the global transition judgement on whether they were dissatisfied (-1), neutral (0) or satisfied (1). The authors felt that OHIP was not a suitable instrument to determine impact of tooth whitening on quality of life.

No other outcomes were reported.

\section{2h. HP paint-on gel versus HP strips}

\section{Tooth whitening - assessed by the dentist}

Two trials, Auschill 2007 using Vita shade guide and Xu 2007 using digital image analysis and $\mathrm{CIEL}^{\star} \mathrm{a}^{*} \mathrm{~b}^{\star}$ scoring showed that HP strips provided superior whitening compared to the paint-on gel group (Xu 2007: MD 1.28, 95\% Cl 0.77 to 1.79; 1 trial, 33 participants; Analysis 19.1); (Auschill 2007: MD 2.70, 95\% Cl 2.08 to 3.32; 1 trial, 40 participants; Analysis 19.2).

\section{Tooth whitening - reported by the patient}

One trial (Auschill 2007) reported no difference in patient satisfaction score between the $5.9 \%$ HP strip and paint-on groups (MD $-0.25,95 \% \mathrm{Cl}-1.88$ to $1.38 ; 1$ trial, 40 participants; Analysis 19.3).

\section{Adverse effects}

Xu 2007 reported that adverse events were mild in severity, and did not contribute to any treatment modification or early withdrawal. Auschill 2007 found a slightly higher tooth hypersensitivity and a slightly higher gingival irritation in the strip group although there was no evidence of a difference between the groups.

No other outcomes were reported.

\section{2i. SPC paint-on versus HP strips}

\section{Tooth whitening - assessed by the dentist}

Bizhang 2007 reported that the 6\% HP strips yielded significant $(P<0.02)$ initial whitening relative to $19 \%$ sodium percarbonate (SPC) paint-on film when measured using digital image analysis and $\mathrm{ClEL}^{*} \mathrm{a}^{*} \mathrm{~b}^{\star}$ scoring (MD $0.93,95 \% \mathrm{Cl} 0.59$ to $1.27 ; 1$ trial, 47 participants; Analysis 20.1).

\section{Adverse effects}

Bizhang 2007 reported tooth sensitivity and oral irritation as the most common adverse events, with strip use. These adverse events were typically symptomatic only, and confined to the treatment period.

No other outcomes were reported.

\section{2j. CP paint-on versus CP paint-on}

Tooth whitening - assessed by the dentist

Two trials included in this comparison used Vita shade guide for measuring shade change.

Li 2004 trial used 18\% CP with different application times. Betweengroup analyses at 7,14 and 21 days showed the means for groups $3 x$ ( 3 times a day) and $4 x$ ( 4 times a day) to be significantly higher than $2 x$ (twice a day) group. However, $3 x$ and $4 x$ groups did not differ significantly ( 7 days; $2 x$ versus $4 x$ : MD $1.39,95 \% \mathrm{Cl} 0.50$ to $2.28 ; 1$ trial, 69 participants; Analysis 21.1).

Brunton 2004 comparing $18 \%$ and $16.4 \%$ CP found both groups with equally effective improvement in whiteness and the difference in whiteness between the two groups was neither statistically nor clinically significant (MD $-0.70,95 \% \mathrm{Cl}-2.21$ to $0.81 ; 1$ trial, 93 participants; Analysis 21.1).

\section{Adverse effects}

Li 2004 reported one subject to have very mild tooth sensitivity on the 7 th day from the 4 times daily application group. Brunton 2004 reported that both gingival and tooth sensitivity were reported to be transient and caused none of the subjects to withdraw from the study.

No other outcomes were reported.

\section{2k. CP paint-on versus HP paint-on}

\section{Tooth whitening - assessed by the dentist}

Nathoo 2003 trial results indicated no evidence of a difference between $25 \% \mathrm{CP}$ and $8.7 \%$ HP paint-on gels, though the shade improved in both groups compared to baseline measured according to the Vita shade scale (MD - $0.16,95 \% \mathrm{Cl}-1.39$ to $1.07 ; 1$ trial, 59 participants; Analysis 22.1).

No other outcomes were reported. 


\section{2l. HP paint-on versus HP-paint-on}

\section{Tooth whitening - assessed by the dentist}

Ziebolz 2008 used Vita shade guide and reported significant improvement in tooth colour, in both groups compared to baseline. However no evidence of a difference was found between the groups (MD - $0.10,95 \% \mathrm{Cl}-0.56$ to $0.36 ; 1$ trial, 67 participants; Analysis 23.1 ).

\section{Patient comfort}

Ziebolz 2008 reported that in both groups similar proportion of the subjects reported lack of comfort. Comfort rating on a 4-point ordinal scale ranging from comfortable to very uncomfortable did not differ significantly.

No other outcomes were reported.

\section{Adverse effects}

Ziebolz 2008 reported tooth sensitivity in both groups with no evidence of a difference.

No other outcomes were reported.

\section{2m. SPC paint-on versus CP paint-on}

\section{Tooth whitening - assessed by the dentist}

Barlow 2003 who used digital image analysis with $\mathrm{CIEL}^{*} \mathrm{a}^{*} \mathrm{~b}^{\star}$ scoring, showed significant and meaningful improvement in tooth colour in 19\% sodium percarbonate group used overnight compared to $18 \%$ CP used twice daily for 7 days and 14 days (14 days: $\mathrm{MD}-0.58,95 \% \mathrm{Cl}-0.95$ to $-0.21 ; 1$ trial, 38 participants; Analysis 24.1).

\section{Adverse effects}

Barlow 2003 reported adverse events related to gum irritation and lip irritation. All events were mild and only one subject in the $18 \%$ $\mathrm{CP}$ group discontinued the treatment.

No other outcomes were reported.

\section{2n. SPC paint-on versus HP paint-on}

\section{Tooth whitening - assessed by the dentist}

Gerlach 2003 used digital image analysis with CIEL*a*b* scoring and reported uniform whitening for all individual and composite colour parameters for both $8.7 \% \mathrm{HP}$ and $19 \%$ SPC groups. Head-tohead clinical testing of these two paint-on gels demonstrated 4-fold greater improvement in composite colour for the 19\% SPC group (MD - $0.36,95 \% \mathrm{Cl}-0.71$ to $-0.01 ; 1$ trial, 56 participants; Analysis 25.1).

\section{Adverse effects}

Gerlach 2003 reported tooth sensitivity in one subject from both groups. One subject in the 19\% SPC film group reported oral sensitivity. All adverse events were symptomatic and mild in severity.

No other outcomes were reported.

\section{DISCUSSION}

\section{Summary of main results}

See Summary of findings for the main comparison; Summary of findings 2; Summary of findings 3; Summary of findings 4; Summary of findings 5; Summary of findings 6; Summary of findings 7; Summary of findings 8; Summary of findings 9; Summary of findings 10; Summary of findings 11; Summary of findings 12; Summary of findings 13; Summary of findings 14; Summary of findings 15; Summary of findings 16; Summary of findings 17; Summary of findings 18; Summary of findings 19.

\section{Bleaching agent versus placebo}

Twenty-six trials compared different whitening products with placebo or no treatment.

Key results for this comparison have been discussed below independently for each outcome.

\section{Key results for the outcome: tooth whitening - assessed by the dentist}

Tray versus placebo: Summary of findings for the main comparison; Summary of findings 2

- Carbamide peroxide (CP) gel in tray at 5\% and $10 \%$ concentrations with varying application times and duration, was more effective than the placebo gel (very low-certainty evidence).

- Hydrogen peroxide (HP) gel in tray at $3 \%$ and $6 \%$ concentrations applied twice daily for 2 weeks, was more effective than the placebo gel (very low-certainty evidence).

\section{Strips versus placebo: Summary of findings 3}

- HP strips at $5.3 \%, 6 \%, 10 \%$ and $14 \%$ concentrations, with varying application times were more effective than the placebo gel (lowand very low-certainty evidence).

Paint-on versus placebo: Summary of findings 4; Summary of findings 5

- CP paint-on gel at $18 \%$ concentration and HP paint-on gel at $6 \%$, applied twice daily for 2 weeks, was more effective than the placebo gel (very low-certainty evidence).

Chewing gum versus placebo: Summary of findings 6; Summary of findings 7

- Sodium hexametaphosphate (SHMP) chewing gum at 4\%, 5.6\% and $7.5 \%$ concentrations, used 4 or 8 times a day showed reduction in stains compared to placebo gum (very low-certainty evidence).

- Sodium tripolyphosphate (STPP) chewing gum at $1 \%$ concentration used 3 times a day showed a reduction in stains compared to placebo gum (very low-certainty evidence).

Mouthrinse versus placebo: Summary of findings 8

- Fluoridated HP mouthwash, improved the shade of the teeth compared to placebo (very low-certainty evidence). 
Key results for the outcome: tooth whitening - reported by the patient

- Patient satisfaction regarding bleaching reported in two trials comparing CP gel in tray to placebo and HP strips to placebo showed more satisfaction in the intervention group.

\section{Key results for the outcome: patient comfort}

- Patient comfort was more for the HP gel in tray group compared to HP strips.

\section{Key results for the outcome: adverse effects}

- Main adverse events reported in most trials were mild and transient tooth sensitivity and oral irritation, which occurred more in the intervention group compared to placebo. However, the placebo group in some trials (tray versus placebo) reported adverse events, which could be due to irritation from the tray.

\section{Key results for the outcome: oral health-related quality of life}

- Oral health-related quality of life (OHRQoL) reported in two trials comparing HP strips to placebo did not show any significant effect of tooth whitening on improvement in quality of life.

\section{Bleaching agent versus bleaching agent}

Fifty-one trials compared one bleaching agent to another bleaching agent.

Key results for this comparison have been discussed below independently for each outcome.

\section{Key results for the outcome: tooth whitening-assessed by the dentist}

Tray versus tray: Summary of findings 9; Summary of findings 10

- Overnight application of two different brands of $10 \%$ CP formulations showed no difference between Opalescence and Nite White (very low-certainty evidence). In another trial, Polanight was shown to have superior whitening compared to Opalescence (very low-certainty evidence). A single trial with 48 participants compared Colgate Platinum and Rembrandt Lighten and showed improved lightening in the CLP group.

- $10 \% \mathrm{CP}$ in tray compared to $15 \% / 16 \% \mathrm{CP}$ in tray favoured the higher concentration group irrespective of duration of use and application time (very low-certainty evidence). However, at the 2-year follow-up no evident difference was found between the groups.

- $10 \% \mathrm{CP}$ with and without desensitisers (potassium nitrate and sodium fluoride) did not show any difference between the groups when used overnight for 2 weeks (very low-certainty evidence). However, $16 \% \mathrm{CP}+$ amorphous calcium phosphate (ACP) showed better whitening when used for 3 hours a day for 2 weeks (low-certainty evidence).

- Overnight application of $5 \%$ and $10 \%$ concentrations of CP did not show any difference in whitening between the groups (very low-certainty evidence).

- Varying concentrations of HP and CP in tray did not show any significant difference at 2 weeks or 12 weeks duration (very lowcertainty evidence). However, one trial showed superiority of $10 \%$ CP in tray over $6 \%$ HP in tray especially for the darkest shade teeth (very low-certainty evidence).
Strip versus tray: Summary of findings 11; Summary of findings 12

- $6 \%$ HP strip was more effective in whitening effect compared to $10 \%$ CP group when tested for a duration of 2 weeks (very lowcertainty evidence). Similar concentrations tested at 6 weeks did not show any difference between the groups (very low-certainty evidence).

- $6.5 \%$ HP strips compared to $16 \%$ CP in tray favoured the CP tray group when tested at 21 days and 3 months duration (lowcertainty evidence). However, one trial comparing $6.5 \% \mathrm{HP}$ to $10 \%$ CP in tray tested for 2 months duration showed better whitening for the strip group.

- Twice daily application of $14 \% \mathrm{HP}$ and $35 \%$ CP did not show any differences in the intergroup comparison (very low-certainty evidence).

- $6.5 \%$ HP strips compared to $15 \%$ CP did not show any difference between the groups (very low-certainty evidence).

- 6\% HP strips showed greater composite colour scores when compared to $5 \%$ CP with desensitiser (very low-certainty evidence).

- $14 \%$ HP strips showed 2-fold increase in lightness compared to 9.5\% HP in tray (very low-certainty evidence).

- $5 \%$ HP strips compared to $5.3 \%$ HP gel in tray showed no difference in whitening between groups (very low-certainty evidence).

\section{Strip versus strip: Summary of findings 13}

- $9.5 \%$ high adhesion HP strips were more effective compared to $10 \%$ HP strips applied for 30 minutes (very low-certainty evidence).

- $10 \% \mathrm{HP}$ strips with a very thin gel were more effective compared to $6 \% \mathrm{HP}$ strips (very low-certainty evidence).

\section{Strip versus mouthwash}

- $10 \% \mathrm{HP}$ whitening strips resulted in significant whitening of teeth compared to $2 \%$ HP mouthrinse used twice daily.

Paint-on versus strips: Summary of findings 14; Summary of findings 15

- $6 \%$ or $5.9 \%$ HP strips showed more improvement in tooth whitening when compared to $18 \% \mathrm{CP}$ or $5.9 \%$ HP paint-on gels with varying application times and duration (very low-certainty evidence). Similar results were obtained when HP strips were compared to $19 \%$ sodium percarbonate paint-on gel.

Paint-on versus paint-on: Summary of findings 16; Summary of findings 17; Summary of findings 18; Summary of findings 19

- No difference was found between $18 \%$ and $16.4 \%$ CP concentrations applied twice daily for 2 weeks (very lowcertainty evidence).

- $18 \%$ CP application 4 times a day was more effective compared to 2 times application (very low-certainty evidence).

- $19 \%$ sodium percarbonate group was more effective in comparison to $18 \%$ CP used twice a day and $8.7 \%$ HP used overnight (very low-certainty evidence).

- HP paint-on with and without desensitiser did not show any difference in tooth whitening. However, these results are from a single trial and cannot be considered with certainty. 
- No difference was found between 25\% CP and 18.5\% HP (very low-certainty evidence).

\section{Key results for the outcome: tooth whitening - reported by the patient}

- Patient contentment for $17 \% \mathrm{CP}$ in tray and $10 \% \mathrm{CP}$ in tray groups were similar with no significant difference (very lowcertainty evidence).

- Patient-reported satisfaction was more for the $5 \%$ HP tray group compared to 5.3\% HP strip group (very low-certainty evidence).

- No significant differences in patient satisfaction was observed for the paint-on and strip groups (very low-certainty evidence). Tooth hypersensitivity and oral irritation were more in the HP strip group with no statistical difference between the paint-on group comparators.

\section{Key results for the outcome: patient comfort}

- Patient comfort was better for the lower concentration group when CP in tray was tested against CP in tray.

- Patient comfort did not vary for $7.5 \% \mathrm{HP}$ in tray and $10 \% \mathrm{CP}$ in tray groups.

- Patient comfort was similar for 6\% HP formulations with and without desensitiser. No significant difference was found in adverse events between any of the comparisons.

\section{Key results for the outcome: adverse effects}

- Higher concentrations of $C P$ in tray led to more tooth sensitivity and gingival irritation. However, the symptoms were mild and transient. CP in tray with desensitiser showed significantly less sensitivity compared to the groups without the desensitiser. No difference was found between HP and CP in tray groups in relation to tooth sensitivity and oral irritation.

- When HP strips were compared to CP gel in tray, results were variable for adverse reactions (tooth sensitivity and oral irritation) with some trials favouring the strip group, some favouring the tray group and some showing no differences between the groups.

- When HP strips were compared to HP strips, very thin gel had lesser tooth sensitivity compared to thicker gel even though the concentration of HP was higher. Strips applied for 2 hours had greater symptoms of sensitivity compared with the 30-minute group. However, these results were not significant.

- Adverse events occurred more in the strip group compared to mouthwash but were not significant.

\section{Key results for the outcome: oral health-related quality of life}

- No difference in OHRQoL was found in $10 \%$ CP in tray compared to $16 \%$ CP in tray.

\section{Overall completeness and applicability of evidence}

\section{Completeness}

We systematically searched for trials according to the methodology written in the protocol. We did an independent Google search and checked all cross references of included articles and other systematic reviews on home-based bleaching to be sure that we did not miss any article. Two pairs of review authors did data extraction in duplicate. Trials, which were not included in the meta-analysis were explained qualitatively. We selected trials with adult participants needing tooth whitening and included all types of interventions with different application methods and concentrations. We included comparisons with placebo as well as head-to-head comparisons. All clinically relevant outcomes of interest were analysed. We also included trials in which staining of teeth was due to tetracycline staining and smoking.

We did not exclude any trial due to missing data. For trials reporting data in graphs, we derived the data by magnifying them and approximating the measures of mean and standard deviation. When mean and standard error (SE) were given, we calculated the standard deviation (SD) as given in the Cochrane Handbook for Systematic Reviews of Interventions section 7.7.3.3 (Higgins 2011). When adjusted mean was given, we considered it in the analysis (Higgins 2011, section 9.2.3.2). When median and interquartile range were given we used the data to calculate mean and SD. When mean and $P$ value were given, SD was calculated. When data were presented as median (skewed data), we qualitatively described the results in the review. When data were presented as odds ratios, log (odds ratio) was calculated based on the odds ratios and $95 \%$ confidence intervals given in the trial and the generic inverse variance method was applied.

\section{Applicability}

Although we had 71 trials (78 reports) included in this review, most of the comparisons were single trials and could not be combined in meta-analyses due to varying methods of application, concentrations, application times, and duration of use. Bleaching agents in any mode of application were shown to be effective compared to placebo though the certainty of evidence is very low. The evidence generated is also of very low quality for most of the comparisons testing a bleaching agent versus another bleaching agent, and hence the results cannot be considered with certainty. Most of the trials report on short-term improvement of shade (ranging from 1 day to 1 month) using home bleaching methods. As follow-up has not been reported in most trials, the results cannot reflect the retention period for the whitening effect. However, the review encourages further high-quality randomised controlled trials (RCTs) to be conducted by standardising methods of application, concentrations, application times, and duration of application.

\section{Quality of the evidence}

The overall certainty of the evidence was low to very low for all comparisons. When a bleaching agent was compared to placebo for the first outcome looking into improvement of shade as measured by the dentist, except for $6 \%$ HP strips compared to placebo, for which evidence was of low certainty, all the other comparisons had a very low-certainty evidence. In the bleaching agent versus bleaching agent comparisons, the evidence for $16 \%$ CP in tray compared to $6.5 \%$ HP strip and 16\% CP with amorphous calcium phosphate compared to $16 \%$ CP was graded as low certainty. The evidence from all remaining studies was graded as very low certainty. Three studies on patient contentment or satisfaction related to whitening treatment, which were included in the metaanalysis were graded as of very low-certainty evidence.

We downgraded the trials mainly for two reasons. Most of the trials were graded serious for risk of bias as they were at an unclear risk of bias and very serious to serious for imprecision as most of the trials were single with limited number of participants and low 
event rates. Few trials that were combined in meta-analysis had high heterogeneity due to which we downgraded the certainty.

\section{Potential biases in the review process}

We have taken steps to minimise bias in every step of the review. We searched all the above mentioned databases, conference proceedings, and trial registries to include all relevant reports. We included foreign language reports in our review. We tried to contact trial authors for missing data through emails, peer contacts, Google search and university/hospital websites where they were previously affiliated. Nevertheless, there could be unpublished data which we could not trace with the above methods. We checked all cross-references in the included articles and other systematic reviews conducted on home-based bleaching and found articles which were missed in the search. Two review authors independently reviewed data extraction forms obtained from translators and cross-checked doubtful areas using the Google translator.

\section{Agreements and disagreements with other studies or reviews}

We found three systematic reviews on home-based bleaching and three conference abstracts.

Niederman 2000 studied only 10\% CP tray-based bleaching products, published between 1989 and 1999 and concluded on the superiority of the intervention over placebo. All the included seven trials have been reported in our review and interpreted similarly.

Gerlach 2007 and Gerlach 2009 studied the efficacy and safety of HP whitening strips against placebo and other controls concluding that strips exhibited superior whitening. All the trials included in these systematic reviews are included in our review and were in agreement with the two systematic reviews.

Three conference abstracts were identified which were systematic reviews of home-based bleaching products. Brennan 2003 included trials with 19\% sodium percarbonate and Gerlach 2013 did a meta-analysis of trials using 10\% HP strips. Gerlach 2010 reported the analysis of peroxide-based self-directed products. We did not compare these with our review due to the lack of complete details.

This Cochrane Review had a broader focus and included all types of bleaching agents and different methods of application compared to the other systematic reviews reported.

\section{AUTHORS' CONCLUSIONS}

\section{Implications for practice}

We found low to very low-certainty evidence over short time periods to support the effectiveness of home-based chemicallyinduced bleaching methods compared to placebo for all the outcomes tested.

We were unable to draw any conclusions regarding the superiority of home-based bleaching compositions or any particular method of application or concentration or application time or duration of use, as the overall evidence generated was of very low certainty. Well-planned randomised controlled trials (RCTs) need to be conducted by standardising methods of application, concentrations, application times, and duration of treatment.

\section{Implications for research}

Further research should be undertaken to know the effectiveness of home-based bleaching methods by conducting well-planned RCTs with more clarity and uniformity in the variables. In designing such clinical trials, the following needs to be considered.

- Evidence: the present evidence was insufficient to conclude that any of the comparisons of home-based bleaching methods are effective. Trials should focus on testing similar concentrations with similar methods of application. Trials should focus on both short-term and long-term benefits of treatment. Studies should also focus on patient-related outcomes and cost effectiveness. Furthermore, reports on clinical trials would be improved by following CONSORT recommendations.

- Population: inclusion criteria for clinical trials should be well defined. Trials should include both genders in equal distribution.

- Intervention: intervention should focus on similar concentrations used in earlier studies and similar application times with a longer follow-up. This will add on to the existing evidence pool allowing us to make robust conclusions.

- Comparison: various comparisons have been reported, but we found only single trials in most of the comparisons due to which the quality of evidence is very low. Hence, RCTs need to be conducted keeping in mind already published studies so that the number of trials for a particular comparison increase.

- Outcome: patient-reported outcomes were not considered in most of the trials. Cost effectiveness also needs to be added in the RCTs, which is of most interest to consumers.

\section{ACKNOWLEDGEMENTS}

We are extremely thankful to Ms Anne Littlewood, Information Specialist; Ms Luisa M Fernandez Mauleffinch, Managing Editor; Dr Philip Riley, Editor; Ms Jo Weldon, Research Co-ordinator; and Prof Helen Worthington, Co-ordinating Editor, Cochrane Oral Health. We thank Prof Datuk Dr Abdul Razzak, Pro Vice Chancellor, Manipal Academy of Higher Education (MAHE), Melaka campus, for his constant encouragement to undertake Cochrane Reviews; Prof Dr Jaspal Singh Sahota, CEO Melaka-Manipal campus for his support; Prof Dr Adinegara Lutfi Abas, Dean, Melaka-Manipal Medical College; and Prof Dr Abdul Rashid Hj Ismail, Dean, Faculty of Dentistry, Melaka-Manipal Medical College for all suggestions and help during the review preparation. The review authors wish to acknowledge the help of translator Dr Annette Blümle, Information Specialist and Researcher, Cochrane Germany; and peer reviewers Dr Alonso Carrasco-Labra, Ms Malavika Tampi, Dr Laura Tam, Dr Adriana Manso and Ms Lesley McGovern Kupiec. We thank Prof Dr Sonal Bakul Joshi, KLE University, Belgaum, India for giving valuable inputs during our review preparation. The authors also wish to thank Prof Dr Pratap Tharyan, Mr Richard K and Mr Jabez Paul from the South Asian Cochrane Network, Vellore, India for their inputs during review completion. We are indebted to Ms Shazana Mohd Selva, Chief Librarian, Melaka-Manipal Medical College; and Ms Janet Lear, School of Dentistry, The University of Manchester. 


\section{R E F E R E N C E S}

\section{References to studies included in this review}

Aka 2017 \{published data only\}

Aka B, Celik EU. Evaluation of the efficacy and color stability of two different at-home bleaching systems on teeth of different shades: a randomized controlled clinical trial. Journal of Esthetic and Restorative Dentistry 2017;29(5):325-38. [PUBMED: 28322505]

\section{Alonso 2006 \{published data only\}}

Alonso de la Peña V, Balboa Cabrita O. Comparison of the clinical efficacy and safety of carbamide peroxide and hydrogen peroxide in at-home bleaching gels. Quintessence International 2006;37(7):551-6. [PUBMED: 16841603]

\section{Alonso 2014 \{published data only\}}

Alonso de la Peña V, López Ratón M. Randomized clinical trial on the efficacy and safety of four professional at-home tooth whitening gels. Operative Dentistry 2014;39(2):136-43. [PUBMED: 23862715]

\section{Auschill 2007 \{published data only\}}

Auschill TM, Barcsay LA, Arweiler NB. Strips versus gel: a clinical comparison of two over-the-counter bleaching systems. Schweizer Monatsschrift fur Zahnmedizin 2007;117(8):843-56. [PUBMED: 17879675]

\section{Auschill 2012 \{published data only\}}

Auschill TM, Schneider-Del Savio T, Hellwig E, Arweiler NB. Randomized clinical trial of the efficacy, tolerability, and longterm color stability of two bleaching techniques: 18-month follow-up. Quintessence International 2012;43(8):683-94. [PUBMED: 23034421]

\section{Barlow 2003 \{published data only\}}

Barlow A, Gerlach RW, Date RF, Brennan K, Struzycka I, Kwiatkowska A, et al. Clinical response of two brush-applied peroxide whitening systems. Journal of Clinical Dentistry 2003;14(3):59-63. [PUBMED: 14520775]

\section{Berga-Caballero 2006 \{published data only\}}

Berga-Caballero A, Forner-Navarro L, Amengual-Lorenzo J. Athome vital bleaching: a comparison of hydrogen peroxide and carbamide peroxide treatments. Medicina Oral, Patologia Oral y Cirugia Bucal 2006;11(1):E94-9. [PUBMED: 16388304]

\section{Biesbrock 2004 \{published data only\}}

Biesbrock AR, Walters P, Bartizek RD. A chewing gum containing $7.5 \%$ sodium hexametaphosphate inhibits stain deposition compared with a placebo chewing gum. Compendium of Continuing Education in Dentistry 2004;25(4):253-4. [PUBMED: 15645861]

\section{Bizhang 2007 \{published data only\}}

Bizhang M, Muller M, Phark JH, Barker ML, Gerlach RW. Clinical trial of long-term color stability of hydrogen peroxide strips and sodium percarbonate film. American Journal of Dentistry 2007;20(A):23A-7A. [PUBMED: 19681255]
Botelho 2017 \{published data only\}

Botelho MG, Chan AWK, Newsome PRH, McGrath CP, Lam WYH. A randomized controlled trial of home bleaching of tetracyclinestained teeth. Journal of Dentistry 2017;67:29-35. [DOI: 10.1016/ j.jdent.2017.05.003; PUBMED: 28478214]

Browning 2008 \{published data only\}

Browning WD, Chan DC, Myers ML, Brackett WW, Brackett MG, Pashley DH. Comparison of traditional and low sensitivity whiteners. Operative Dentistry 2008;33(4):379-85. [PUBMED: 18666494]

\section{Bruhn 2012 \{published data only\}}

Bruhn AM, Darby ML, McCombs GB, Lynch CM. Vital tooth whitening effects on oral health-related quality of life in older adults. Journal of Dental Hygiene 2012;86(3):239-47. [PUBMED: 22947847]

\section{Brunton 2004 \{published data only\}}

Brunton PA, Ellwood R, Davies R. A six-month study of two self-applied tooth whitening products containing carbamide peroxide. Operative Dentistry 2004;29(6):623-6. [PUBMED: 15646216]

\section{Cibirka 1999 \{published data only\}}

Cibirka RM, Myers M, Downey MC, Nelson SK, Browning WD, Hawkins IK, et al. Clinical study of tooth shade lightening from dentist-supervised, patient-applied treatment with two $10 \%$ carbamide peroxide gels. Journal of Esthetic Dentistry 1999;11(6):325-31. [PUBMED: 10825867]

\section{Collins 2004a \{published data only\}}

Collins LE, Maggio B, Liebman J, Blanck M, Lefort S, Waterfield P, et al. Clinical evaluation of a novel whitening gel, containing $6 \%$ hydrogen peroxide and a standard fluoride toothpaste. Journal of Dentistry 2004;32(Suppl 1):13-7. [PUBMED: 14738830]

Costa 2012 \{published data only\}

da Costa JB, McPharlin R, Hilton T, Ferracane JI, Wang M. Comparison of two at-home whitening products of similar peroxide concentration and different delivery methods. Operative Dentistry 2012;37(4):333-9. [PUBMED: 22433035]

\section{Cronin 2005 \{published data only\}}

Cronin MJ, Charles CA, Zhao Q, Dembling WZ. Comparison of two over-the-counter tooth whitening products using a novel system. Compendium of Continuing Education in Dentistry 2005;26(2):140, 142, 144-8. [PUBMED: 15948518]

\section{Delgado 2007 \{published data only\}}

Delgado E, Hernandez-Cott PL, Stewart B, Collins M, De Vizio W. Tooth-whitening efficacy of custom tray-delivered $9 \%$ hydrogen peroxide and $20 \%$ carbamide peroxide during daytime use: a 14-day clinical trial. Puerto Rico Health Sciences Journal 2007;26(4):367-72. [PUBMED: 18246965]

\section{Ferrari 2007 \{published data only\}}

Ferrari M, Cagidiaco MC, Monticelli F, Kugel G, Barker ML, Gerlach RW. Daytime use of a custom bleaching tray or 
whitening strips: initial and sustained color improvement. American Journal of Dentistry 2007;20 Spec No A:19A-22A. [PUBMED: 19681254]

\section{Gallo 2009 \{published data only\}}

Gallo JR, Burgess JO, Ripps AH, Bell MJ, Mercante DE, Davidson JM. Evaluation of 30\% carbamide peroxide at-home bleaching gels with and without potassium nitrate - a pilot study. Quintessence International 2009;40(4):1-6. [PUBMED: 19417866]

\section{Garcia-Godoy 2004 \{published data only\}}

Garcia-Godoy F, Villalta P, Barker ML, Gerlach RW. Placebocontrolled, 6-week clinical trial on the safety and efficacy of a low-gel, 14\% hydrogen-peroxide whitening strip. Compendium of Continuing Education in Dentistry 2004;25(8 Suppl 2):21-6. [PUBMED: 15645891]

\section{Gerlach 2000 \{published data only\}}

Gerlach RW, Gibb RD, Sagel PA. A randomized clinical trial comparing a novel $5.3 \%$ hydrogen peroxide whitening strip to $10 \%, 15 \%$, and $20 \%$ carbamide peroxide tray-based bleaching systems. Compendium of Continuing Education in Dentistry 2000;29:S22-8; S42-3. [PUBMED: 11908406]

\section{Gerlach 2002 \{published data only\}}

Gerlach RW, Gibb RD, Sagel PA. Initial color change and color retention with a hydrogen peroxide bleaching strip. American Journal of Dentistry 2002;15(1):3-7. [PUBMED: 12074226]

\section{Gerlach 2002a \{published data only\}}

Gerlach RW, Zhou X. Comparative clinical efficacy of two professional bleaching systems. Compendium of Continuing Education in Dentistry 2002;23(1A):35-41. [PUBMED: 11913292]

\section{Gerlach 2002b \{published data only\}}

Gerlach RW, Zhou X, McClanahan SF. Comparative response of whitening strips to a low peroxide and potassium nitrate bleaching gel. American Journal of Dentistry 2002;15:19A-23A. [PUBMED: 12512987]

\section{Gerlach 2003 \{published data only\}}

Gerlach RW, Barker ML. Randomized clinical trial comparing overnight use of two self-directed peroxide tooth whiteners. American Journal of Dentistry 2003;16 Spec No:17B-21B. [PUBMED: 15055983]

\section{Gerlach 2004 \{published data only\}}

Gerlach RW, Zhou X. Clinical trial comparing two daytime hydrogen-peroxide professional vital-bleaching systems. Compendium of Continuing Education in Dentistry 2004;25(8 Suppl 2):33-40. [PUBMED: 15645893]

\section{Gerlach 2004e \{published data only\}}

Gerlach RW, Sagel PA, Barker ML, Karpinia KA, Magnusson I. Placebo-controlled clinical trial evaluating a 10\% hydrogen peroxide whitening strip. Journal of Clinical Dentistry 2004;15(4):118-22. [PUBMED: 15794457]

\section{Gerlach 2005 \{published data only\}}

Gerlach RW, Tucker HL, Anastasia MK, Barker ML. Clinical trial comparing 2 hydrogen peroxide tooth whitening systems: strips versus pre-rinse. Compendium of Continuing Education in Dentistry 2005;26(12):874-8. [PUBMED: 16389774]

Giniger 2005 \{published data only\}

Giniger M, Macdonald J, Ziemba S, Felix H. The clinical performance of professionally dispensed bleaching gel with added amorphous calcium phosphate. Journal of the American Dental Association 2005;136(3):383-92. [PUBMED: 15819354]

* Giniger M, Spaid M, MacDonald J, Felix H. A 180-day clinical investigation of the tooth whitening efficacy of a bleaching gel with added amorphous calcium phosphate. Journal of Clinical Dentistry 2005;16(1):11-6. [PUBMED: 15974218]

\section{Hannig 2007 \{published data only\}}

Hannig C, Lindner D, Attin T. Efficacy and tolerability of two home bleaching systems having different peroxide delivery. Clinical Oral Investigations 2007;11(4):321-9. [PUBMED: 17593406]

\section{Hasturk 2004 \{published data only\}}

Hasturk H, Nunn M, Warbington M, Van Dyke TE. Efficacy of a fluoridated hydrogen peroxide-based mouthrinse for the treatment of gingivitis: a randomised clinical trial. Journal of Periodontology 2004;75(1):57-65. [PUBMED: 15025217 ]

\section{Hyland 2015 \{published data only\}}

Hyland BW, McDonald A, Lewis N, Tredwin C, Petrie A, Hall S, et al. A new three-component formulation for the efficient whitening of teeth (Carbamide Plus). Clinical Oral Investigations 2015;19(6):1395-404. [PUBMED: 25381018 ]

\section{Karpinia 2002 \{published data only\}}

Karpinia KA, Magnusson I, Sagel PA, Zhou X, Gerlach RW. Vital bleaching with two at-home professional systems. American Journal of Dentistry 2002;15:13A-18A. [PUBMED: 12512986]

\section{Kihn 2000 \{published data only\}}

Kihn PW, Barnes DM, Romberg E, Peterson K. A clinical evaluation of 10 per cent versus 15 per cent carbamide peroxide tooth - whitening agents. Journal of the American Dental Association 2000;131:1478-84. [PUBMED: 11042989]

\section{Kose 2011 \{published data only\}}

Kose C, Reis A, Baratieri LN, Loguercio AD. Clinical effects of athome bleaching along with desensitizing agent application. American Journal of Dentistry 2011;24(6):379-82. [PUBMED: 22263337]

\section{Kowitz 1994 \{published data only\}}

Kowitz GM, Nathoo SA, Wong R. Comparative clinical evaluation of two professional tooth-whitening products. Compendium (Newtown, Pa). Supplement 1994;15(17):S635-9. [PUBMED: 8205580]

\section{Krause 2008 \{published data only\}}

Krause F, Jepsen S, Braun A. Subjective intensities of pain and contentment with treatment outcomes during tray 
bleaching of vital teeth employing different carbamide peroxide concentrations. Quintessence International 2008;39(3):203-9. [PUBMED: 18618034]

\section{Kugel 2000 \{published data only\}}

Kugel G, Kastali S. Tooth-whitening efficacy and safety: a randomized and controlled clinical trial. Compendium of Continuing Education in Dentistry 2000;21(29):S16-21; S42. [PUBMED: 11908405]

\section{Kugel 2002 \{published data only\}}

Kugel G, Aboushala A, Zhou X, Gerlach RW. Daily use of whitening strips on tetracycline-stained teeth: comparative results after 2 months. Compendium of Continuing Education in Dentistry 2002;23(1A):29-34. [PUBMED: 11913291]

\section{Li 2003 \{published data only\}}

Li Y, Lee SS, Cartwright SL, Wilson AC. Comparison of clinical efficacy and safety of three professional at-home tooth whitening systems. Compendium of Continuing Education in Dentistry 2003;24(5):357-60. [PUBMED: 12793220]

\section{Li 2004 \{published data only\}}

Li Y, Lee SS, Cartwright S, Wilson AC, DeVizio W, Petrone M, et al. Comparative tooth whitening efficacy of $18 \%$ carbamide peroxide liquid whitening gel using three different regimens. Journal of Clinical Dentistry 2004;15(1):11-6. [PUBMED: 15218710]

\section{Matis 1998 \{published data only\}}

Matis BA, Cochran MA, Eckert G, Carlson TJ. The efficacy and safety of a $10 \%$ carbamide peroxide bleaching gel. Quintessence International 1998;29(9):555-65. [PUBMED: 9807138]

\section{Matis 2000 \{published data only\}}

Matis BA, Mousa HN, Cochran MA, Eckert GJ. Clinical evaluation of bleaching agents of different concentrations. Quintessence International 2000;31(5):303-10. [PUBMED: 11203940]

\section{Matis 2006 \{published data only\}}

* Matis BA, Wang Y, Eckert GJ, Cochran MA, Jiang T. Extended bleaching of tetracycline-stained teeth: a 5-year study. Operative Dentistry 2006;31(6):643-51. [PUBMED: 17153971 ]

Matis BA, Wang Y, Jiang T, Eckert GJ. Extended at-home bleaching of tetracycline-stained teeth with different concentrations of carbamide peroxide. Quintessence International 2002;33(9):645-55. [PUBMED: 12666888]

\section{Mederios 2008 \{published data only\}}

dos Santos Medeiros MC, de Lima KC. Effectiveness of nightguard vital bleaching with $10 \%$ carbamide peroxide a clinical study. Journal of the Canadian Dental Association 2008;74(2):163-163e. [PUBMED: 18353201]

\section{Meireles 2010 \{published data only\}}

Meireles SS, Goettems ML, Dantas RVF, Bona AD, Santos IS, Demarco FF. Changes in oral health related quality of life after dental bleaching in a double-blind randomized clinical trial. Journal of Dentistry 2014;42:114-21. [PUBMED: 24316342]
Meireles SS, Heckmann SS, Leida FL, Santos IS, Bona AD, Demarco FF. Efficacy and safety of $10 \%$ and $16 \%$ carbamide peroxide tooth-whitening gels: a randomized clinical trial. Operative Dentistry 2008;33(6):606-12. [PUBMED: 19051852 ]

Meireles SS, Heckmann SS, Santos IS, Bona AD, Demarco FF. A double blind randomized clinical trial of at-home tooth bleaching using two carbamide peroxide concentrations: 6 month follow-up. Journal of Dentistry 2008;36(11):878-84. [PUBMED: 18722039]

* Meireles SS, Santos IS, Bona AD, Demarco FF. A doubleblind randomized clinical trial of two carbamide peroxide tooth bleaching agents: 2-year follow-up. Journal of Dentistry 2010;38(12):956-63. [PUBMED: 20709137]

Meireles SS, Santos IS, Bona AD, Demarco FF. A double-blind randomized controlled clinical trial of 10 percent versus 16 percent carbamide peroxide tooth-bleaching agents. Oneyear follow-up. Journal of the American Dental Association 2009;140(9):1109-17. [PUBMED: 19723943]

Meireles SS, Santos IS, Della BA, Demarco FF. Randomized clinical trial of two tooth bleaching agents: 2-year followup. IADR/AADR/CADR General Session; 2009 Apr 1-4; Miami (Florida). 2009. [2367]

\section{Mohan 2008 \{published data only\}}

Mohan N, Westland S, Brunton P, Ellwood R, Pretty IA, Luo W. A clinical study to evaluate the efficacy of a novel tray based tooth whitening system. Journal of Dentistry 2008;36(1):21-6. [PUBMED: 18006206 ]

\section{Mokhlis 2000 \{published data only\}}

Mokhlis GR, Matis BA, Cochran MA, Eckert GJ. A clinical evaluation of carbamide peroxide and hydrogen peroxide whitening agents during daytime. Journal of the American Dental Association 2000;131(9):1269-77. [PUBMED: 10986827]

Myers 2003 \{published data only\}

Myers ML, Browning WD, Downey MC, Hackman ST. Clinical evaluation of a $3 \%$ hydrogen peroxide tooth-whitening gel. Journal of Esthetic and Restorative Dentistry 2003;15(1):50-6. [PUBMED: 12638773]

\section{Nathoo 1994 \{published data only\}}

Nathoo SA, Chmielewski MB, Rustogi KN. Clinical evaluation of Colgate Platinum Professional Toothwhitening System and Rembrandt Lighten Bleaching Gel. Compendium (Newtown, Pa). Supplement 1994;14(17):S640-5. [PUBMED: 8205581]

\section{Nathoo 2001 \{published data only\}}

Nathoo S, Santana E 3rd, Zhang YP, Lin N, Collins M, Klimpel K, et al. Comparative seven-day clinical evaluation of two tooth whitening products. Compendium of Continuing Education in Dentistry 2001;22(7):599-604; 606; 608. [PUBMED: 11494621]

\section{Nathoo 2002 \{published data only\}}

Nathoo S, Stewart B, Zhang YP, Chaknis P, Rustogi KN, Devizio W, et al. Efficacy of a novel, nontray, paint-on $18 \%$ carbamide peroxide whitening gel. Compendium of Continuing 
Education in Dentistry 2002;23(11 Suppl 1):26-31. [PUBMED: 12789994]

\section{Nathoo 2003 \{published data only\}}

Nathoo S, Stewart B, Petrone ME, Chaknis P, Zhang YP, DeVizio W, et al. Comparative clinical investigation of the tooth whitening efficacy of two tooth whitening gels. Journal of Clinical Dentistry 2003;14(3):64-9. [PUBMED: 14520776]

\section{Navarra 2014 \{published data only\}}

Navarra CO, Reda B, Diolosa M, Casula I, Di Lenarda R, Breschi L, et al. The effects of two $10 \%$ carbamide peroxide nightguard bleaching agents, with and without desensitizer, on enamel and sensitivity: an in vivo study. International Journal of Dental Hygiene 2014;12(2):115-20. [PUBMED: 24119064]

Oliveira 2013 \{published data only\} Oliveira GM, Miguez PA, Oliveira GB, Swift EJ Jr, Farrell S, Anastasia MK, et al. Safety and efficacy of a high-adhesion whitening strip under extended wear regimen. Journal of Dentistry 2013;41 Suppl 3:e46-52. [PUBMED: 23228500]

\section{Papas 2009 \{published data only\}}

Papas AS, Kugel G, Singh M, Barker ML, Gerlach RW. Placebocontrolled clinical trial of use of $10 \%$ hydrogen peroxide whitening strips for medication-induced xerostomia. Gerontology 2009;55(5):511-6. [PUBMED: 19707010]

\section{Porciani 2006 \{published data only\}}

Porciani PF, Grandini S, Perra C, Grandini R. Whitening effect by stain inhibition from a chewing gum with sodium hexametaphosphate in a controlled twelve-week single-blind trial. Journal of Clinical Dentistry 2006;17(1):14-6. [PUBMED: 16838876]

\section{Porciani 2010 \{published data only\}}

Porciani PF, Perra C, Grandini S. Effect on dental stain occurrence by chewing gum containing sodium tripolyphosphate - A double-blind six-week trial. Journal of Clinical Dentistry 2010;21(1):4-7. [PUBMED: 20527505]

\section{Russell 1996 \{published data only\}}

Russell CM, Dickinson GL, Johnson MH, Curtis JW Jr, Downey MC, Haywood VB, et al. Dentist-supervised home bleaching with ten percent carbamide peroxide gel: a six-month study. Journal of Esthetic Dentistry 1996;8(4):177-82. [PUBMED: 9468838]

\section{Shahidi 2005 \{published data only\}}

Shahidi H, Barker ML, Sagel PA, Gerlach RW. Randomized controlled trial of $10 \%$ hydrogen peroxide whitening strips. Journal of Clinical Dentistry 2005;16(3):91-5. [PUBMED: 16305009]

\section{Swift 2004 \{published data only\}}

Swift EJ Jr, Miguez PA, Barker ML, Gerlach RW. Three-week clinical trial of a $14 \%$ hydrogen-peroxide, strip-based bleaching system. Compendium of Continuing Education in Dentistry 2004;25(8 Suppl 2):27-32. [PUBMED: 15645892]
Swift 2009 \{published data only\}

Swift EJ Jr, Heymann HO, Wilder AD Jr, Barker ML, Gerlach RW. Effects of duration of whitening strip treatment on tooth color: a randomized, placebo-controlled clinical trial. Journal of Dentistry 2009;37 Suppl 1:e51-6. [PUBMED: 19523738]

Tam 2001 \{published data only\}

Tam L. Effect of potassium nitrate and fluoride on carbamide peroxide bleaching. Quintessence International 2001;32(10):766-70. [PUBMED: 11820045]

\section{Tsubura 2005 \{published data only\}}

Tsubura S, Yamaguchi R. Clinical evaluation of a new bleaching product 'Polanight' in a Japanese population. Odontology 2005;93(1):52-5. [PUBMED: 16170477]

Turkun 2010 \{published data only\}

Turkun M, Celik EU, Aladag A, Gokay N. One-year clinical evaluation of the efficacy of a new daytime at-home bleaching technique. Journal of Esthetic and Restorative Dentistry 2010;22(2):139-48. [PUBMED: 20433566 ]

\section{Walters 2004 \{published data only\}}

Walters PA, Biesbrock AR, Bartizek RD. Benefits of sodium hexametaphosphate containing chewing gum for extrinsic stain inhibition. Journal of Dental Hygiene 2004;78(4):1-7. [PUBMED: 16197748]

\section{Wong 2004 \{published data only\}}

Wong AHH. A Randomized Controlled Trial of Home ToothWhitening Products [Dissertation]. The University of Hong Kong 2004.

\section{Xu 2007 \{published data only\}}

Xu X, Zhu L, Tang Y, Wang Y, Zhang K, Li S, et al. Randomized clinical trial comparing whitening strips, paint-on gel and negative control. American Journal of Dentistry 2007;20:28A-31A. [PUBMED: 19681256]

\section{Ziebolz 2007 \{published data only\}}

Ziebolz D, Helms K, Hannig C, Attin T. Efficacy and oral side effects of two highly concentrated tray-based bleaching systems. Clinical Oral Investigations 2007;11(3):267-75. [PUBMED: 17333304 ]

\section{Ziebolz 2008 \{published data only\}}

Ziebolz D, Hannig C, Attin T. Influence of a desensitizing agent on efficacy of a paint-on bleaching agent. American Journal of Dentistry 2008;21(2):77-82. [PUBMED: 18578172]

\section{References to studies excluded from this review}

\section{Alkmin 2005 \{published data only\}}

Alkmin YT, Sartorelli R, Florio FM, Basting RT. Comparative study of the effects of two bleaching agents on oral microbiota. Operative Dentistry 2005;30(4):417-23. [PUBMED: 16130860]

Amini 2009 \{published data only\}

Amini P, Qaqish J, Anastasia MK, Hamilton AJ, Gerlach RW, Farrell S. Clinical response of experimental whitening strips with 
6-weeks daily use. IADR/AADR/CADR General Session; 2009 Apr 1-4; Miami (Florida). 2009. [2277]

\section{Amini 2011 \{published data only\}}

Amini P, Qaqish J, Conde E, Anastasia MK, Widmeyer V, Farrell S. Immediate effects and color degradation profile of 2-hour whitening strip. IADR/AADR/CADR General Session; 2011 March 16-19; San Diego (California). 2011. [2539]

\section{Anastasia 2010 \{published data only\}}

Anastasia MK, Conde E, Widmeyer V, Fiedler S, Farrell S. Clinical efficacy and safety of two marketed whitening treatments. AADR/CADR Annual Meeting; 2010 March 3-6; Washington (DC). 2010. [447]

\section{Andreana 2000 \{published data only\}}

Andreana S, Ciancio SG, Mather ML. Clinical evaluation of bleaching gels on patients with sensitive teeth. Journal of Dental Research 2000;79(1 Suppl):216 (Abs No 583).

\section{Archila 2010 \{published data only\}}

Archila L, Ibanez E, Conde E, Widmeyer V, Anastasia MK, Farrell S. Short-term whitening efficacy of marketed highadhesion whitening strips. AADR/CADR Annual Meeting; 2010 March 3-6; Washington (DC). 2010. [833]

\section{Auschill 2009 \{published data only\}}

Auschill TM, Schneider-del ST, Hellwig E, Arweiler NB. Clinical comparison of two different bleaching systems: tray versus strips. IADR/AADR/CADR General Session; 2009 Apr 1-4; Miami (Florida). 2009. [2832]

\section{Browning 2001 \{published data only\}}

Browning WD, Myers ML, Downey MC, Pohjola RM, Hackman ST, Cliett BA. Safety and efficacy of an experimental tooth whitening agent. Journal of Dental Research 2001;80(1 Suppl):182 (Abs No 1176).

\section{Burgio 2001 \{published data only\}}

Burgio PA, Donovan TE, Kugel G, Matranga LF. Multi-center clinical study to compare the effectiveness of dental bleaching. Journal of Dental Research 2001;80(1 Suppl):150 (Abs No 918).

\section{Cardoso 2010 \{published data only\}}

Cardoso PC, Reis A, Loguercio A, Vieira LC, Baratieri LN. Clinical effectiveness and tooth sensitivity associated with different bleaching times for a 10 percent carbamide peroxide gel. Journal of the American Dental Association 2010;141(10):1213-20. [PUBMED: 20884923]

\section{Collins 2004 \{published data only\}}

Collins LZ, Maggio B, Gallagher A, York M, Schafer F. Safety evaluation of a novel whitening gel, containing $6 \%$ hydrogen peroxide and a commercially available whitening gel containing $18 \%$ carbamide peroxide in an exaggerated use clinical study. Journal of Dentistry 2004;32(1):47-50. [PUBMED: 14738835]

\section{Corby 2014 \{published data only\}}

Corby PM, Biesbrock A, Gerlach R, Corby AL, Moreira A, Schork NJ, et al. Treatment responses to tooth whitening in twins. Twin Research and Human Genetics 2014;17(1):23-6. [PUBMED: 24429255]

\section{Curtis 1996 \{published data only\}}

Curtis JW, Dickinson GL, Downey MC, Russell CM, Haywood VB, Myers ML, et al. Assessing the effects of 10 percent carbamide peroxide on oral soft tissues. Journal of the American Dental Association 1996;127(8):1218-23. [PUBMED: 8803398]

\section{de Geus 2015a \{published data only\}}

de Geus JL, Rezende M, Margraf LS, Bortoluzzi MC, Fernandez E, Loguercio $A D$, et al. Evaluation of genotoxicity and efficacy of at-home bleaching in smokers: a single-blind controlled clinical trial. Operative Dentistry 2015;40(2):E47-55. [PUBMED: 25535783]

\section{de Geus 2015b \{published data only\}}

de Geus JL, Bersezio C, Urrutia J, Yamada T, Fernandez E, Loguercio AD, et al. Effectiveness of and tooth sensitivity with at-home bleaching in smokers: a multicenter clinical trial. Journal of the American Dental Association 2015;146(4):233-40. [PUBMED: 25819654]

\section{Dickinson 2000 \{published data only\}}

Dickinson GL, Browning WD, Hawkins IK, Downey MC, Myers ML, Hackman ST, et al. Clinical evaluation of ADS tooth whitening gel. Journal of Dental Research 2000;79(1 Suppl):438 (Abs No 2359).

\section{Donly 2001 \{published data only\}}

Donly KJ, Gerlach R, Segura A, Walters P, Zhou X. Postorthodontic tooth whitening. Journal of Dental Research 2001;80(1 Suppl):151 (Abs No 924).

\section{Donly 2002 \{published data only\}}

Donly KJ, Donly AS, Baharloo L, Rojas-Candelas E, GarciaGodoy F, Zhou X, et al. Tooth whitening in children. Compendium of Continuing Education in Dentistry 2002;23(1A):22-8. [PUBMED: 11913290]

Donly 2002a \{published data only\} Donly KJ, Gerlach RW. Clinical trials on the use of whitening strips in children and adolescents. General Dentistry 2002;50(3):242-5. [PUBMED: 12116511]

\section{Farrell 2006 \{published data only\}}

Farrell S, Barker ML, Sagel PA, Gerlach RW. Use of a physical barrier to improve efficacy of a paint-on whitening gel: a sevenday randomized clinical trial. Journal of Clinical Dentistry 2006;17(5):117-21. [PUBMED: 17240929]

\section{Farrell 2008 \{published data only\}}

Farrell S, Barker ML, McMillan DA, Gerlach RW. Placebocontrolled trial evaluating safety with 12 -months continuous use of $6 \%$ hydrogen peroxide whitening strips. Journal of Dentistry 2008;36(9):726-30. [PUBMED: 18635304]

Fugaro 2004 \{published data only\}

Fugaro JO, Nordahl I, Fugaro OJ, Matis BA, Mjor IA. Pulp reaction to vital bleaching. Operative Dentistry 2004;29(4):363-8. [PUBMED: 15279473] 
Fugaro 2005 \{published data only\}

Fugaro OJ, Fugaro JO, Matis B, Gregory RL, Cochran MA, Mjor I. The dental pulp: inflammatory markers and vital bleaching. American Journal of Dentistry 2005;18(4):229-32. [PUBMED: 16296427]

\section{Garcia-Godoy 2012 \{published data only\}}

Garcia-Godoy C, Truehart D, Rothrock JK, Farrell S, Anastasia MK, Conde E, et al. Whitening efficacy and color degradation profile of 2-hour whitening strips. AADR/CADR Annual Meeting; 2012 March 21-24; Tampa (Florida). 2012. [478]

\section{Gerlach 2002c \{published data only\}}

Gerlach RW, Sagel PA, Jeffers ME, Zhou X. Effect of peroxide concentration and brushing on whitening clinical response. Compendium of Continuing Education in Dentistry 2002;23(1A):16-21. [PUBMED: 11913289]

\section{Gerlach 2002d \{published data only\}}

Gerlach RW, Gibb RD, Zhou X. Clinical response of maxillary and mandibular teeth following use of $6.5 \%$ hydrogen peroxide whitening strips. Journal of Dental Research 2002;81(1 Suppl):A-253 (Abs No 1954).

\section{Gerlach 2002e \{published data only\}}

Gerlach RW, Campolongo KL, Zhou X. Use of a chroma meter to compare the shade change for two professional tooth whitening systems. Journal of Dental Research 2002;81(1 Suppl):A-253 (Abs No 1957).

\section{Gerlach 2003a \{published data only\}}

Gerlach RW, Barker ML. Clinical response of three directto-consumer whitening products: strips, paint-on gel, and dentifrice. Compendium of Continuing Education in Dentistry 2003;24(6):458, 461-4, 466. [PUBMED: 14515596]

\section{Gerlach 2004a \{published data only\}}

Gerlach RW, Barker ML, Tucker HL. Clinical response of three whitening products having different peroxide delivery: comparison of tray, paint-on gel, and dentifrice. Journal of Clinical Dentistry 2004;15(4):112-7. [PUBMED: 15794456]

\section{Gerlach 2004c \{published data only\}}

Gerlach RW, Barker ML, McMillan DA, Sagel PA, Walden GL. In-use comparative kinetics of professional whitening strips: peroxide recovery from strips, teeth, gingiva, and saliva. Compendium of Continuing Education in Dentistry 2004;25(8 Suppl 2):14-20. [PUBMED: 15645890]

\section{Gerlach 2004d \{published data only\}}

Gerlach RW, Barker ML. Professional vital bleaching using a thin and concentrated peroxide gel on whitening strips: an integrated clinical summary. Journal of Contemporary Dental Practice 2004;5(1):1-17. [PUBMED: 14973556]

\section{Godson 2001 \{published data only\}}

Goodson JM, Taveres M, Stutz J, Liao SS, Kent R, Newman M. Colorimeter measurement of tooth whitening. Journal of Dental Research 2001;80(1 Suppl):182 (Abs No 1170). [1170]

\section{Gursoy 2008 \{published data only\}}

Gursoy UK, Eren DI, Bektas OO, Hurmuzlu F, Bostanci V, Ozdemir H. Effect of external tooth bleaching on dental plaque accumulation and tooth discoloration. Medicina Oral, Patologia Oral y Cirugia Bucal 2008;13(4):E266-9. [PUBMED: 18379454]

Jadad 2011 \{published data only\}

Jadad E, Montoya J, Arana G, Gordillo LA, Palo RM, Loguercio AD. Spectrophotometric evaluation of color alterations with a new dental bleaching product in patients wearing orthodontic appliances. American Journal of Orthodontics and Dentofacial Orthopedics 2011;140(1):e43-7. [PUBMED: 21724070]

Jorgensen 2002 \{published data only\} Jorgensen MG, Carroll WB. Incidence of tooth sensitivity after home whitening treatment. Journal of the American Dental Association 2002;133(8):1076-82. [PUBMED: 12198987]

Karpinia 2003 \{published data only\} Karpinia K, Magnusson I, Barker ML, Gerlach RW. Clinical comparison of two self-directed bleaching systems. Journal of Prosthodontics 2003;12(4):242-8. [PUBMED: 15061232]

Lee 2003 \{published data only\}

Lee BJ, Paik DI, Kim JB. Clinical evaluation of the toothwhitening effect of a $2.6 \%$ hydrogen peroxide tooth-whitening strip. AADR/CADR Anual Meeting; 2003 March; San Antonio (Texas). 2003. [1876]

Leonard 2002 \{published data only\}

Leonard RH Jr, Garland GE, Eagle JC, Caplan DJ. Safety issues when using a $16 \%$ carbamide peroxide whitening solution. Journal of Esthetic and Restorative Dentistry 2002;14(6):358-67. [PUBMED: 12542101]

\section{Leonard 2004 \{published data only\}}

Leonard RH, Smith LR, Garland GE, Caplan DJ. Densensitizing Agent Efficacy during Whitening in an At Risk Population. Journal of Esthetic and Restorative Dentistry 2004;16(1):49-56. [PUBMED: 15259543]

\section{Leonard 2007 \{published data only\}}

Leonard RH Jr, Smith LR, Garland GE, Tiwana KK, Zaidel LA, Pugh G Jr, et al. Evaluation of side effects and patients' perceptions during tooth bleaching. Journal of Esthetic and Restorative Dentistry 2007;19(6):355-64; discussion 365-6. [PUBMED: 18005286]

\section{Lisante 2009 \{published data only\}}

Lisante TA, Askinazi J, Simmons K, Charles C, Mcguire JT. Comparative whitening effectiveness of three hydrogen peroxide-containing mouthrinses. IADR/AADR/CADR General Session; 2009 Apr 1-4; Miami (Florida). 2009. [2597]

Loyola-Rodriguez 2003 \{published data only\}

Loyola-Rodriguez JP, Pozos-Guillen Ade J, HernandezHernandez F, Berumen-Maldonado R, Patino-Marin N. Effectiveness of treatment with carbamide peroxide and hydrogen peroxide in subjects affected by dental 
fluorosis: a clinical trial. Journal of Clinical Pediatric Dentistry 2003;28(1):63-7. [PUBMED: 14604145]

Majeed 2011 \{published data only\}

Majeed A, Grobler SR, Moola MH. Clinical evaluation of two athome tooth-whitening products using a spectrophotometer. 45th CED-IADR/NOF Meeting; 2011 Aug 31; Budapest (Hungary). 2011. [231]

\section{Marques 2012 \{published data only\}}

Marques DN, da Mata AD, Silveira JM, Marques JR, Amaral JP, Guilherme NF. Hydrogen peroxide release kinetics into saliva from different whitening products: a double-blind, randomized clinical trial. Clinical Oral Investigations 2012;16(1):155-63. [PUBMED: 21221681]

\section{Martin 2015 \{published data only\}}

Martin J, Vildosola P, Bersezio C, Herrera A, Bortolatto J, Saad JR, et al. Effectiveness of $6 \%$ hydrogen peroxide concentration for tooth bleaching - A double-blind, randomized clinical trial. Journal of Dentistry 2015;43(8):965-72. [PUBMED: 26057085]

\section{Matis 1999 \{published data only\}}

Matis BA, Gaiao U, Blackman D, Schultz FA, Eckert GJ. In vivo degradation of bleaching gel used in whitening teeth. Journal of the American Dental Association 1999;130(2):227-35. [PUBMED: 10036846]

\section{Matis 2002 \{published data only\}}

Matis BA, Yousef M, Cochran MA, Eckert GJ. Degradation of bleaching gels in vivo as a function of tray design and carbamide peroxide concentration. Operative Dentistry 2002;27(1):12-8. [PUBMED: 11817465]

\section{Matis 2002a \{published data only\}}

Matis BA, Hamdan YS, Cochran MA, Eckert GJ. A clinical evaluation of a bleaching agent used with and without reservoirs. Operative Dentistry 2002;27(1):5-11. [PUBMED: 11817468]

\section{Matis 2005 \{published data only\}}

Matis BA, Cochran M, Wang G, Franco M, Eckert GJ, Carlotti RJ, et al. A clinical evaluation of bleaching using whitening wraps and strips. Operative Dentistry 2005;30(5):588-92. [PUBMED: 16268392]

\section{Mazur 2013 \{published data only\}}

Mazur RF, Busato P, Souza EM, Vieira S, Marini A, Rached R. Effect of aloe vera on the adverse effects of bleaching. IADR/ AADR/CADR General Session; 2013 March 20-23; Seattle (Washington). 2013. [1144]

\section{NCT02603354 \{published data only\}}

NCT02603354. Efectiveness of a brief protocol by a low (6\%) concentration gel peroxide hydrogen of teeth bleaching (brief 6\%). clinicaltrials.gov/ct2/show/NCT02603354 (first received 11 November 2015).
NCT02682329 \{unpublished data only\}

NCT02682329. Combination of dental bleaching techniques, randomized clinical trial. clinicaltrials.gov/ct2/show/ NCT02682329 (first received 15 February 2016).

\section{Perdigao 2013 \{published data only\}}

Perdigao J, Dutra-Correa M, Saraceni CHC, Delazari MA, Kodama RM, Bergamini MR. Clinical evaluation of $10 \%$ carbamide peroxide with different desensitizers. IADR/AADR/ CADR General Session; 2013 March 20-23; Seattle (Washington). 2013. [603]

\section{Pinto 2014 \{published data only\}}

Pinto MM, de Godoy CH, Bortoletto CC, Olivan SR, Motta LJ, Altavista OM, et al. Tooth whitening with hydrogen peroxide in adolescents: study protocol for a randomized controlled trial. Trials 2014;15:395. [PUBMED: 25315893]

\section{Pinto 2017 \{published data only\}}

Pinto MM, Goncalves ML, Mota AC, Deana AM, Olivan SR, Bortoletto $\mathrm{C}$, et al. Controlled clinical trial addressing teeth whitening with hydrogen peroxide in adolescents: a 12month follow-up. Clinics (Sao Paulo, Brazil) 2017;72(3):161-70. [PUBMED: 28355362]

\section{Rezende 2013 \{published data only\}}

Rezende M, Loguercio AD, Reis A, Kossatz S. Clinical effects of exposure to coffee during at-home vital bleaching. Operative Dentistry 2013;38(6):E229-36. [PUBMED: 23570297]

\section{Sagel 2001 \{published data only\}}

Sagel PA, Walters PA, Gibb RD, Gerlach RW. Duration of tooth whitening following 14 days treatment with peroxidecontaining whitening strips. Journal of Dental Research 2001;80(1 Suppl):182 (Abs No 1173).

Schiff 1994 \{published data only\}

Schiff T, Borden LC. The effect of a new experimental prebrushing dental rinse on plaque removal. Journal of Clinical Dentistry 1994;4(4):107-10. [PUBMED: 8031477]

\section{Schulte 1993 \{published data only\}}

Schulte JR, Morrissette DB, Gasior EJ, Czajewski MV. Clinical changes in the gingiva as a result of at-home bleaching. Compendium 1993;14(11):1362, 1364-6. [PUBMED: 8620374]

\section{Schulte 1994 \{published data only\}}

Schulte JR, Morrissette DB, Gasior EJ, Czajewski MV. The effects of bleaching application time on the dental pulp. Journal of the Americal Dental Association 1994;125(10):1330-5. [PUBMED: 7844297]

\section{Simon 2001 \{published data only\}}

Simon JF, McClanahan SF, Gerlach RW. Clinical trial comparing tooth whitening with peroxide-containing strips to a marketed whitening dentifrice. Journal of Dental Research 2001;80(1 Suppl):237 (Abs No 1616).

\section{Simon 2011 \{published data only\}}

Simon JF, Lewis M, Powell L, Wilson N, Conde E, Anastasia MK, et al. Efficacy of 2-hour whitening strip under extended use 
conditions. IADR/AADR/CADR General Session; 2011 March 16-19; San Diego (California). 2011. [2540]

\section{Smith 2001 \{published data only\}}

Smith LR, Leonard RH, Eagle JC, Murdoch WC, Garland GE, Caplan DJ. Efficacy of desensitizing gel in reducing tooth sensitivity during whitening. Journal of Dental Research 2001;80(1 Suppl):246 (Abs No 1683).

\section{Swift 2001 \{published data only\}}

Swift EJ Jr, Heymann HO, Ritter AV, Rosa BT, Wilder AD Jr. Clinical evaluation of a novel 'trayless' tooth whitening system. Journal of Dental Research 2001;80(1 Suppl):151 (Abs No 921).

Tam 1999 \{published data only\}

Tam L. Clinical trial of three $10 \%$ carbamide peroxide bleaching products. Journal of the Canadian Dental Association 1999;65(4):201-5. [PUBMED: 10224721]

Walter 2011 \{published data only\}

Walter R, Miguez PA, Oliveira GCB, Oliveira GMS, Farrell S, Anastasia MK, et al. Safety and efficacy of a 2-hour whitening strip. IADR/AADR/CADR General Session; 2011 March 16-19; San Diego (California). 2011. [555]

Yankell 1997 \{published data only\}

Yankell SL. Efficacy of chewing gum in preventing extrinsic tooth staining. Journal of Clinical Dentistry 1997;8(6):169-72. [PUBMED: 9586534]

\section{Zantner 2006 \{published data only\}}

Zantner C, Derdilopoulou F, Martus P, Kielbassa AM. Randomized clinical trial on the efficacy of 2 over-the-counter whitening systems. Quintessence International 2006;37:695-706. [PUBMED: 17017631]

\section{References to studies awaiting assessment}

\section{Barnes 1998 \{published data only\}}

Barnes DM, Kihn PW, Romberg E, George D, DePaola L, Medina E. Clinical evaluation of a new $10 \%$ carbamide peroxide tooth-whitening agent. Compendium of Continuing Education in Dentistry 1998;19(10):968-72, 977-8. [PUBMED: 10371880]

\section{Bizhang 2017 \{published data only\}}

Bizhang M, Domin J, Danesh G, Zimmer S. Effectiveness of a new non-hydrogen peroxide bleaching agent after single use a double-blind placebo-controlled short-term study. Journal of Applied Oral Science 2017;25(5):575-84. [PUBMED: 29069156]

\section{Braun 2007 \{published data only\}}

Braun A, Jepsen S, Krause F. Spectrophotometric and visual evaluation of vital tooth bleaching employing different carbamide peroxide concentrations. Dental Materials 2007;23(2):165-9. [PUBMED: 16504281]

\section{Browning 2004 \{published data only\}}

Browning WD, Chan DC, Frazier KB, Callan RS, Blalock JS. Safety and efficacy of a nightguard bleaching agent containing sodium fluoride and potassium nitrate. Quintessence International 2004;35(9):693-8. [PUBMED: 15470992]

Ferrari 2004 \{published data only\}

Ferrari M, Kugel G, Cagidiaco MC, Barker ML, Gerlach RW. Clinical trial evaluating the peroxide concentration response of whitening strips over 28 days. American Journal of Dentistry 2004;17(4):291-4. [PUBMED: 15478494]

Gambarini 2004 \{published data only\}

Gambarini G, Testarelli L, De Luca M, Dolci G. Efficacy and safety assessment of a new liquid tooth whitening gel containing $5.9 \%$ hydrogen peroxide. American Journal of Dentistry 2004;17(2):75-9. [PUBMED: 15151330]

\section{Gegauff 1993 \{published data only\}}

Gegauff AG, Rosenstiel SF, Langhout KJ, Johnston WM. Evaluating tooth color change from carbamide peroxide gel. Journal of the American Dental Association 1993;124(6):65-72. [PUBMED: 8505452]

\section{Gerlach 2004b \{published data only\}}

Gerlach RW, Sagel PA. Vital bleaching with a thin peroxide gel: the safety and efficacy of a professional-strength hydrogen peroxide whitening strip. Journal of the American Dental Association 2004;135(1):98-100. [PUBMED: 14959882]

\section{Guerrero 2007 \{published data only\}}

Guerrero JC, Jimenez-Farfan MD, Lopez-Salgado A, Barker ML, Gerlach RW. Professional whitening strips in a university population. American Journal of Dentistry 2007;20 Spec No A:15A-8A. [PUBMED: 19681253

\section{Heymann 1998 \{published data only\}}

Heymann HO, Swift EJ Jr, Bayne SC, May KN Jr, Wilder AD Jr, Mann GB, et al. Clinical evaluation of two carbamide peroxide tooth-whitening agents. Compendium of Continuing Education in Dentistry 1998;19(4):359-62, 364-6. [PUBMED: 9656849]

\section{Kim 2018 \{published data only\}}

Kim YM, Ha AN, Kim JW, Kim SJ. Double-blind randomized study to evaluate the safety and efficacy of over-the-counter toothwhitening agents containing $2.9 \%$ hydrogen peroxide. Operative Dentistry 2018;43(3):272-81. [PUBMED: 29513635]

\section{Maran 2018 \{published data only\}}

Maran BM, Vochikovski L, de Andrade Hortkoff DR, Stanislawczuk R, Loguercio AD, Reis A. Tooth sensitivity with a desensitizing-containing at-home bleaching gel - a randomized triple-blind clinical trial. Journal of Dentistry 2018;72:64-70. [PUBMED: 29551346]

\section{NCT02151058 \{published data only\}}

NCT02151058. A clinical trial to test the effect of a marketed mouth rinse on stain removal [Whitening action of a hydrogen peroxide/sodium fluoride containing mouth rinse: a 2 week randomized controlled trial]. clinicaltrials.gov/ct2/show/ NCT02151058 (first received 30 May 2014). 


\section{NCT03217994 \{published data only\}}

NCT03217994. Efficacy of a $6 \%$ hydrogen peroxide tooth bleaching agent [Efficacy of a $6 \%$ hydrogen peroxide tooth bleaching agent without ligth]. clinicaltrials.gov/ct2/show/ NCT03217994 (first received 14 July 2017).

\section{Ozcan 2003 \{published data only\}}

Ozcan M, Kulak Y, Kazazoglu E. The efficacy of two prototype chewing gums for the removal of extrinsic tooth stain. International Dental Journal 2003;53(2):62-6. [PUBMED: 12731691]

\section{Pohjola 2002 \{published data only\}}

Pohjola RM, Browning WD, Hackman ST, Myers ML, Downey MC. Sensitivity and tooth whitening agents. Journal of Esthetic and Restorative Dentistry 2002;14(2):85-91. [PUBMED: 12008806]

Reinhardt 1993 \{published data only\}

Reinhardt JW, Eivins SE, Swift EJ Jr, Denehy GE. A clinical study of nightguard vital bleaching. Quintessence International 1993;24(6):379-84. [PUBMED: 8234642]

\section{Rosenstiel 1996 \{published data only\}}

Rosenstiel SF, Gegauff AG, Johnston WM. Randomized clinical trial of the efficacy and safety of a home bleaching procedure. Quintessence International 1996;27(6):413-24. [PUBMED: 8941836]

\section{Rossi 2018 \{published data only\}}

Rossi B, Freitas PM, Tedesco TK, Goncalves F, Ferreira LS. Tooth color changes and sensitivity in patients undergoing dental bleaching with $10 \%$ hydrogen peroxide using customized trays or strips: a randomized clinical trial. Minerva Stomatologica 2018;67(2):55-61. [PUBMED: 29446268]

\section{Shin 2010 \{published data only\}}

Shin BG, Yang SE. The evaluation of clinical efficacy and longevity of home bleaching without combined application of in office bleaching. Journal of Korean Academy of Conservative Dentistry 2010;35(5):387-94.

\section{Sielski 2003 \{published data only\}}

Sielski C, Conforti N, Stewart B, Chaknis P, Petrone ME, DeVizio W, et al. A clinical investigation of the efficacy of a toothwhitening gel. Compendium of Continuing Education in Dentistry 2003;24(8):612-4, 616-8. [PUBMED: 14692166]

\section{Simon 2014 \{published data only\}}

Simon JF, Powell L, Hollis S, Anastasia MK, Gerlach RW, Farrell S. Placebo-controlled clinical trial evaluating 9.5\% hydrogen peroxide high-adhesion whitening strips. Journal of Clinical Dentistry 2014;25(3):49-52. [PUBMED: 26054177]

\section{References to ongoing studies}

NCT03026725 \{published data only\}

NCT03026725. Effect of tri calcium phosphate on efficacy and sensitivity with Vital tooth whitening using $20 \%$ carbamide peroxide [Effect of tri calcium phosphate paste on efficacy and postoperative sensitivity associated with at-home Vital tooth whitening using $20 \%$ carbamide peroxide - A randomized controlled study]. clinicaltrials.gov/ct2/show/NCT03026725 (first received 20 January 2017).

\section{Additional references \\ Alkhatib 2004}

Alkhatib MN, Holt R, Bedi R. Prevalence of self-assessed tooth discolouration in the United Kingdom. Journal of Dentistry 2004;32(7):561-6. [PUBMED: 15386863]

\section{Alqahtani 2014}

Alqahtani MQ. Tooth-bleaching procedures and their controversial effects: a literature review. Saudi Dental Journal 2014;26(2):33-46. [PUBMED: 25408594]

\section{Balshem 2011}

Balshem H, Helfand M, Schünemann HJ, Oxman AD, Kunz R, Brozek J, et al. GRADE guidelines: 3 . Rating the quality of evidence. Journal of Clinical Epidemiology 2011;64(4):401-6. [PUBMED: 21208779]

\section{Barabolak 1991}

Barabolak R, Hoerman K, Kroll B, Record D. Gum chewing profiles in the US population. Community Dentistry and Oral Epidemiology 1991;19(2):125-6. [PUBMED: 2049920]

\section{Baroudi 2014}

Baroudi K, Hassan NA. The effect of light-activation sources on tooth bleaching. Nigerian Medical Journal 2014;55(5):363-8. [PUBMED: 25298598]

\section{Belobrov 2011}

Belobrov I, Parashos P. Treatment of tooth discoloration after the use of white mineral trioxide aggregate. Journal of Endodontics 2011;37(7):1017-20. [PUBMED: 21689563]

\section{Brennan 2003}

Brennan K, Barker ML, Zhou X, Barlow AP, Gerlach RW. Factors contributing to tooth whitening with a direct application of percarbonate bleaching film: meta-analysis of 7 clinical trials. IADR/PER General Session; 2003 June; Goteborg (Sweden). 2003. [1047]

\section{Caballero 2006}

Berga-Caballero A, Forner-Navarro L, Amengual-Lorenzo J. Athome vital bleaching: a comparison of hydrogen peroxide and carbamide peroxide treatments. Medicina Oral, Patologia Oral y Cirugia Bucal 2006;11(1):94-9. [PUBMED: 16388304]

\section{Carey 2014}

Carey CM. Tooth whitening: what we now know. Journal of Evidence-based Dental Practice 2014;14:70-6. [PUBMED: 24929591]

\section{Curtin 2002}

Curtin F, Elbourne D, Altman DG. Meta-analysis combining parallel and cross-over clinical trials. II: Binary outcomes. Statistics in Medicine 2002;21(15):2145-59. 


\section{Dantas 2015}

Dantas AAR, Bortolatto JF, Roncolato A, Merchan H, Floros MC, Kuga MC, et al. Can a bleaching toothpaste containing Blue Covarine demonstrate the same bleaching as conventional techniques? An in vitro, randomized and blinded study. Journal of Applied Oral Science 2015;23(6):609-13.

\section{de Vasconcelos 2012}

de Vasconcelos AA, Cunha AG, Borges BC, Machado CT, dos Santos AJ. Tooth whitening with hydrogen/carbamide peroxides in association with a CPP-ACP paste at different proportions. Australian Dental Journal 2012;57(2):213-9.

\section{Deeks 2011}

Deeks JJ, Higgins JP, Altman DG, editor(s). Chapter 9: Analysing data and undertaking meta-analyses. In: Higgins JP, Green S, editor(s). Cochrane Handbook for Systematic Reviews of Interventions Version 5.1.0 (updated March 2011). The Cochrane Collaboration, 2011. Available from handbook.cochrane.org.

\section{Demarco 2009}

Demarco FF, Meireles SS, Masotti AS. Over-the-counter whitening agents: a concise review. Brazilian Oral Research 2009;23:64-70. [PUBMED: 19838560]

\section{Duschner 2006}

Duschner H, Götz H, White DJ, Kozak KM, Zoladz JR. Effects of hydrogen peroxide bleaching strips on tooth surface color, surface microhardness, surface and subsurface ultrastructure, and microchemical (Raman spectroscopic) composition. Journal of Clinical Dentistry 2006;17(3):72-8. [PUBMED: 17022369]

\section{Egger 1997}

Egger M, Davey Smith G, Schneider M, Minder C. Bias in meta-analysis detected by a simple, graphical test. $B M J$ 1997;315(7109):629-34. [PUBMED: 9310563]

\section{Gerlach 2001}

Gerlach RW, Barker ML, Sagel PA. Comparative efficacy and tolerability of two direct-to-consumer tooth whitening systems. American Journal of Dentistry 2001;14(5):267-72. [PUBMED: 11803987]

\section{Gerlach 2007}

Gerlach RW. Tooth whitening clinical trials: a global perspective. American Journal of Dentistry 2007;20 Spec No A:3A-6A. [PUBMED: 19681251]

\section{Gerlach 2009}

Gerlach RW, Barker ML, Karpinia K, Magnusson I. Single site meta-analysis of $6 \%$ hydrogen peroxide whitening strip effectiveness and safety over 2 weeks. Journal of Dentistry 2009;37(5):360-5. [PUBMED: 19233534]

\section{Gerlach 2010}

Gerlach RW, Anastasia MK, Farrell S, Barker ML, Sagel PA. Safety and efficacy meta-analysis of self-directed tooth whitening by delivery. AADR/CADR Annual Meeting; 2010 March 3-6; Washington (DC). 2010. [828]

\section{Gerlach 2013}

Gerlach RW, Barker ML, Anastasia MK, Farrell S, Sagel PA. Subject-level meta-analysis of $10 \%$ hydrogen peroxide whitening strip clinical response. IADR/AADR/CADR General Session; 2013 March 20-23; Seattle (Washington). 2013. [27]

\section{Goldstein 1995}

Goldstein RE, Graber DA. Complete Dental Bleaching. 1st Edition. Chicago: Quintessence Publishing, 1995:73-4.

\section{GRADEpro GDT 2015 [Computer program]}

McMaster University (developed by Evidence Prime). GRADEpro GDT. Version accessed 12 June 2018. Hamilton (ON): McMaster University (developed by Evidence Prime), 2015.

\section{Haywood 1992}

Haywood VB. History, safety, and effectiveness of current bleaching techniques and applications of the nightguard vital bleaching technique. Quintessence International 1992;23(7):471-88. [PUBMED: 1410249]

\section{Higgins 2011}

Higgins JPT, Green S, editor(s). Cochrane Handbook for Systematic Reviews of Interventions Version 5.1.0 (updated March 2011). The Cochrane Collaboration, 2011. Available from handbook.cochrane.org.

\section{Horn 2014}

Horn BA, Bittencourt BF, Gomes OM, Farhat PA. Clinical evaluation of the whitening effect of over-the-counter dentifrices on vital teeth. Brazilian Dental Journal 2014;25(3):203-6. [PUBMED: 25252254]

\section{Javaheri 2000}

Javaheri DS, Janis JN. The efficacy of reservoirs in bleaching trays. Operative Dentistry 2000;25(3):149-51.

\section{Joiner 2006}

Joiner A. The bleaching of teeth: a review of the literature. Journal of Dentistry 2006;34(7):412-9. [PUBMED: 16569473]

\section{Joshi 2016}

Joshi SB. An overview of vital teeth bleaching. Journal of Interdisciplinary Dentistry 2016;6(1):3-13. [DOI: 10.4103/2229-5194.188155]

\section{Kim 2010}

Kim JH, Kim Y, Shin SJ, Park JW, Jung IY. Tooth discoloration of immature permanent incisor associated with triple antibiotic therapy: a case report. Journal of Endodontics 2010;36(6):1086-91. [PUBMED: 20478471]

\section{Kolosowski 2014}

Kolosowski KP, Sodhi RN, Kishen A, Basrani BR. Qualitative analysis of precipitate formation on the surface and in the tubules of dentin irrigated with sodium hypochlorite and a final rinse of chlorhexidine or QMiX. Journal of Endodontics 2014;40(12):2036-40. [PUBMED: 25305239] 


\section{Kowitz 1994a}

Kowitz GM, Nathoo SA, Rustogi KN, Chmielewski MB, Liang LJ, Wong R. Clinical comparison of Colgate Platinum Tooth Whitening System and Rembrandt Gel Plus. Compendium (Newtown, Pa). Supplement 1994;15(17):S646-51. [PUBMED: 8205582]

\section{Kwon 2015}

Kwon SR, Wertz PW. Review of the mechanism of tooth whitening. Journal of Esthetic and Restorative Dentistry 2015;27(5):240-57. [PUBMED: 25969131]

\section{Lefebvre 2011}

Lefebvre C, Manheimer E, Glanville J. Chapter 6: Searching for studies. In: Higgins JP, Green S, editor(s). Cochrane Handbook for Systematic Reviews of Interventions Version 5.1.0 (updated March 2011). The Cochrane Collaboration, 2011. Available from handbook.cochrane.org.

\section{Leonard 2003}

Leonard RH Jr, Van Haywood B, Caplan DJ, Tart ND. Nightguard vital bleaching of tetracycline-stained teeth: 90 months post treatment. Journal of Esthetic and Restorative Dentistry 2003;15(3):142-52. [PUBMED: 12859112]

\section{Lima 2012}

Lima FG, Rotta TA, Penso S, Meireles SS, Demarco FF. In vitro evaluation of the whitening effect of mouth rinses containing hydrogen peroxide. Brazilian Oral Research 2012;26(3):269-74. [PUBMED: 22641448]

\section{Mankodi 2001}

Mankodi SM, Conforti N, Berkowitz H. Efficacy of baking soda-containing chewing gum in removing natural tooth stain. Compendium of Continuing Education in Dentistry 2001;22(7A):29-32. [PUBMED: 11913307]

\section{Nathoo 1997}

Nathoo SA. The chemistry and mechanisms of extrinsic and intrinsic discoloration. Journal of the American Dental Association 1997;128:6-10. [PUBMED: 9120149]

\section{Niederman 2000}

Niederman R, Tantraphol MC, Slinin P, Hayes C, Conway S. Effectiveness of dentist-prescribed, home-applied tooth whitening. A meta-analysis. Journal of Contemporary Dental Practice 2000;1(4):20-36. [PUBMED: 12167948]

\section{Panich 2001}

Panich M. In Vivo Evaluation of 15-Percent Carbamide Peroxide and 5.5-Percent Hydrogen Peroxide Whitening Agents During Daytime Use [Masters thesis]. Indiana (USA): School of Dentistry, Indiana University, 2001.

\section{Perdigão 2004}

Perdigão J, Baratieri LN, Arcari GM. Contemporary trends and techniques in tooth whitening: a review. Practical Procedures and Aesthetic Dentistry 2004;16(3):185-92. [PUBMED: 15199693]

\section{Po 2014}

Po LH, Wilson NW. Effects of different desensitizing agents on bleaching treatments. European Journal of General Dentistry 2014;3(2):93-9.

\section{Review Manager 2014 [Computer program]}

Nordic Cochrane Centre, The Cochrane Collaboration. Review Manager 5 (RevMan 5). Version 5.3. Copenhagen: Nordic Cochrane Centre, The Cochrane Collaboration, 2014.

\section{Schünemann 2011}

Schünemann HJ, Oxman AD, Vist GE, Higgins JP, Deeks JJ, Glasziou P, et al. Chapter 12: Interpreting results and drawing conclusions. In: Higgins JP, Green S, editor(s). Cochrane Handbook for Systematic Reviews of Interventions Version 5.1.0 (updated March 2011). The Cochrane Collaboration, 2011. Available from handbook.cochrane.org.

\section{Shellis 2005}

Shellis RP, Addy M, Rees GD. In vitro studies on the effect of sodium tripolyphosphate on the interactions of stain and salivary protein with hydroxyapatite. Journal of Dentistry 2005;33(4):313-24. [PUBMED: 15781139]

\section{Soparkar 2001}

Soparkar P, Newman MB. Effects of a baking soda gum on extrinsic dental stain: results of a longitudinal 4-week assessment. Compendium of Continuing Education in Dentistry 2001;22(7A):25-8. [PUBMED: 11913306]

\section{Viscio 2000}

Viscio D, Gaffar A, Fakhry-Smith S, Xu T. Present and future technologies of tooth whitening. Compendium of Continuing Education in Dentistry 2000;28:36-43. [PUBMED: 11908346]

\section{White 2002}

White DJ. A new and improved 'dual action' whitening dentifrice technology - sodium hexametaphosphate. Journal of Clinical Dentistry 2002;13(1):1-5. [PUBMED: 11507924]

\section{Whitening survey 2012}

American Academy of Cosmetic Dentistry. Whitening survey. www.aacd.com/proxy/files/Publications\%20and\%20Resources/ Whitening\%20Survey_Aug12(1).pdf (accessed prior to 3 July 2018).

\section{References to other published versions of this review Hasson 2006}

Hasson H, Ismail A, Neiva G. Home-based chemicallyinduced whitening of teeth in adults (Review). Cochrane Database of Systematic Reviews 2006, Issue 4. [DOI: 10.1002/14651858.CD006202]

* Indicates the major publication for the study 
CHARACTERISTICS OF STUDIES

Characteristics of included studies [ordered by study ID]

Aka 2017

Methods

Title: evaluation of the efficacy and colour stability of 2 different at-home bleaching systems on teeth of different shades

Trial design: randomised, parallel-group, controlled clinical trial

Location: Izmir Katip Celebi University, Turkey

Language: English

Number of centres: 1

Recruitment period: January to July 2014

Funding source: not reported

Participants

Participants: 20 to 51 years old, mean age 26 years

Total number: 200

Inclusion criteria:

- good general health

- adults $18+$

- no caries or restoration on the teeth

- permanent teeth

- availability for follow-up

- at least 1 front teeth from each shade category

Exclusion criteria:

- history of allergy

- tetracycline stain

- poor oral hygiene

- pregnant or lactating women

- having parafunctional habits

- current or previous use of bleaching

- orthodontic treatment

- hypersensitivity

Number randomised: 92 (all patients had similar number of light, medium dark and dark shaded teeth)

Method of randomisation: randomisation table

Method of allocation concealment: not reported

Method of blinding: not reported

Number evaluated: 90

Group 1: placebo $n=31$

Group 2: 10\% carbamide peroxide gel (Opalescence PF) $n=30$

Group 3: 6\% hydrogen peroxide gel (Opalescence Go) $n=31$ 
Aka 2017 (Continued)

Duration of treatment: 14 days

Each group was further divided based on the shade (tooth level analysis)

Control group: light teeth $n=90$, medium dark teeth $n=91$, dark teeth $n=93$

Experiment Group 2: light teeth $n=89$, medium dark $n=81$, dark teeth $n=83$

Experiment Group 3: light teeth $n=91$, medium teeth $n=88$, dark teeth $n=81$

$\begin{array}{ll}\text { Outcomes } & \text { Shade evaluation (dental spectrophotometer); tooth sensitivity, gingival irritation and patient satisfac- } \\ \text { tion (self-assessed using a 7-point scale: } 1 \text { correlating to no sensitivity, no problems, or no satisfaction; } & 7 \text { correlating to severe sensitivity, problems, or satisfaction }\end{array}$

Sample size calculation: given
Adverse effects: tooth sensitivity and irritation
Key conclusions of the study authors: "A pre-loaded tray system may be used for dental bleaching, but
it is still less effective than conventional 10\% carbamide peroxide system, irrespective of the initial
shade. Bleaching was more effective with dark teeth compared to light teeth. Patient satisfaction was
higher in 10\% CP group compared to 6\% HP"
Correspondence required: no
Contact: Department of Restorative Dentistry, Faculty of Dentistry, Izmir Katip Celebi University, Izmir,
Turkey

\section{Risk of bias}

\begin{tabular}{|c|c|c|}
\hline Bias & Authors' judgement & Support for judgement \\
\hline $\begin{array}{l}\text { Random sequence genera- } \\
\text { tion (selection bias) }\end{array}$ & Low risk & $\begin{array}{l}\text { Quote: "The patients were randomly assigned to one of the two treatment } \\
\text { groups and a control group using a randomization table..." }\end{array}$ \\
\hline $\begin{array}{l}\text { Allocation concealment } \\
\text { (selection bias) }\end{array}$ & Unclear risk & Not mentioned \\
\hline $\begin{array}{l}\text { Blinding of participants } \\
\text { and personnel (perfor- } \\
\text { mance bias) } \\
\text { All outcomes }\end{array}$ & Unclear risk & Not mentioned \\
\hline $\begin{array}{l}\text { Blinding of outcome as- } \\
\text { sessment (detection bias) } \\
\text { All outcomes }\end{array}$ & High risk & $\begin{array}{l}\text { Assessed as at high risk of bias due to the lack of clarity of how teeth were se- } \\
\text { lected for outcome assessment }\end{array}$ \\
\hline $\begin{array}{l}\text { Incomplete outcome data } \\
\text { (attrition bias) } \\
\text { All outcomes }\end{array}$ & Low risk & $\begin{array}{l}\text { Quote: "2 patients from } 6 \% \text { HP/Go groups with } 6 \text { light shades of teeth did not } \\
\text { attend the } 6 \text { months visit" } \\
\text { Comment: Plausible effect size (difference in means) among missing outcomes } \\
\text { not enough to have a clinically relevant impact on observed effect size }\end{array}$ \\
\hline $\begin{array}{l}\text { Selective reporting (re- } \\
\text { porting bias) }\end{array}$ & Low risk & $\begin{array}{l}\text { All outcomes described were reported. Conclusions are in accordance with the } \\
\text { results }\end{array}$ \\
\hline Other bias & Low risk & None \\
\hline
\end{tabular}




\section{Methods}

Title: comparison of the clinical efficacy and safety of carbamide peroxide and hydrogen peroxide in athome bleaching gels

Trial design: split-mouth randomised controlled trial

Location: not reported

Language: English

Number of centres: 1

Recruitment period: not reported

Funding source: not reported

Participants

Participants: 18 to 50 years, mean age: 31.8 years

Total number: 16

Inclusion criteria:

- subject availability to attend the control visits

- good overall general health

- a minimum of 24 natural teeth, including at least 4 molars (excluding third molars)

- willingness to refrain from the use of any type of mouthrinse during participation in the study

Exclusion criteria:

- medication; need of antibiotic prophylactic therapy to receive dental treatment

- smoking habit

- pregnant or breastfeeding

- presence of active cavities; anterior sector restorations covering $>1 / 6$ of the labial surface

- anterior teeth with root canal treatment

- crowns or veneers on anterior teeth

- a Löe-Silness Gingival Index 25 above 1

- stains confirmed to be due to tetracycline

Number randomised: 16

Method of randomisation: not reported

Method of allocation concealment: not reported

Method of blinding: not reported

Number evaluated: 16

Interventions

Total number of intervention groups: 2

$3.5 \%$ hydrogen peroxide gel $+5 \%$ potassium nitrate

$10 \%$ carbamide peroxide gel

Duration of treatment: 3 hours a day on each arch, renew the gel every hour for 4 weeks

Dental sensitivity: a scale of 4 levels, absence of sensitivity (grade 0 ), slight sensitivity not necessitating suspension of treatment (grade 1), sensitivity that forced suspension of treatment for 1 day (grade 2), sensitivity that led to suspension of treatment for more than 1 day (grade 3 ) 
Alonso 2006 (Continued)

Gingival irritation: present or absent

Sample size calculation: not reported
Adverse effects: sensitivity
Health-related quality of life: not reported
Key conclusions of the study authors: "Under the conditions of this study, no statistically significant dif-
ferences were detected between 3.5\% hydrogen peroxide containing 5\% potassium nitrate (FKD) and
the $10 \%$ carbamide peroxide-based product (Opalescence)"
Correspondence required: no
Contact: Victor Alonso de la Peña; victorap@mundo-r.com

\section{Risk of bias}

\begin{tabular}{lll}
\hline Bias & Authors' judgement & Support for judgement \\
\hline $\begin{array}{l}\text { Random sequence genera- } \\
\text { tion (selection bias) }\end{array}$ & Unclear risk & Quote: "Both groups applied the products on a random basis" \\
\hline $\begin{array}{l}\text { Allocation concealment } \\
\text { (selection bias) }\end{array}$ & Unclear risk & Not mentioned \\
\hline $\begin{array}{l}\text { Blinding of participants } \\
\text { and personnel (perfor- } \\
\text { mance bias) }\end{array}$ & Unclear risk & Not mentioned \\
$\begin{array}{l}\text { All outcomes } \\
\text { Blinding of outcome as- } \\
\text { sessment (detection bias) } \\
\text { All outcomes }\end{array}$ & Unclear risk & Not mentioned \\
\hline $\begin{array}{l}\text { Incomplete outcome data } \\
\text { (attrition bias) }\end{array}$ & Low risk & All 16 patients completed the study \\
$\begin{array}{l}\text { All outcomes } \\
\begin{array}{l}\text { Selective reporting (re- } \\
\text { porting bias) }\end{array}\end{array}$ & Low risk & All outcomes described were reported. Conclusions are in accordance with the \\
\hline $\begin{array}{l}\text { Other bias } \\
\text { results }\end{array}$ & None \\
\hline
\end{tabular}

\section{Alonso 2014}

\section{Methods}

Title: randomised clinical trial on the efficacy and safety of 4 professional at home tooth whitening gels

Trial design: randomised controlled clinical trial

Location: Faculty of Medicine and Dentistry, Santiago de Compostela, Spain

Language: English

Number of centres: 1

Recruitment period: not reported

Funding source: not reported 
Alonso 2014 (Continued)

Participants
Participants: mean age 25.9 years

Total number: 96

Inclusion criteria:

- 18 years and older

- minimum 24 natural teeth, with incisor, canine and premolars in both arches

- absence of periodontal disease and gingival recession

- availability to complete the study

Exclusion criteria:

- systemic illness

- antibiotic prophylaxis

- pregnant and breastfeeding women

- tumours of hard and soft palate

- presence of restoration

- xerostomia, smokers

- fluoride supplements and desensitizing agents

- removable prosthesis

- previous history of bleaching

Number randomised: 96

Method of randomisation: based on alphabetical order

Method of allocation concealment: not reported

Method of blinding: not reported

Number evaluated: 96
Total number of intervention groups: 4

$10 \%$ carbamide peroxide

$15 \%$ carbamide peroxide

$7.5 \%$ hydrogen peroxide

9.5\% hydrogen peroxide

Duration of treatment: 2 weeks

\section{Outcomes}

Change in colour

Vita shade guide arranged from lightest to darkest B1 (1) to C4 (16). And change in score was recorded

$\Delta \mathrm{L}, \mathrm{a}^{\star}, \mathrm{b}^{\star}$ were recorded $\left(\mathrm{b}^{\star}:\right.$ decreased $\mathrm{b}$ indicates reduced yellowness; $\Delta \mathrm{L}$ : increased $\Delta \mathrm{L}$ is increased brightness)

Sensitivity: 0 = none, 1 = mild, 2 = moderate, $3=$ considerable, $4=$ severe

$\begin{array}{ll}\text { Notes } & \text { Sample size calculation: not reported } \\ \text { Adverse effects: sensitivity } \\ \text { Health-related quality of life: not reported }\end{array}$


Alonso 2014 (Continued)

Key conclusions of the study authors: "There were no differences in the degree of whitening among the different products. With all of the products there was an increase in $L^{*}$, a decrease in chromatic intensity $\left(\mathrm{C}^{\star}\right)$, and an increase in the value (tone) or hue $\left(\mathrm{h}^{\star}\right) "$

Contact: Dr Teijeiro; victorap@mundo-r.com

\title{
Risk of bias
}

\begin{tabular}{|c|c|c|}
\hline Bias & Authors' judgement & Support for judgement \\
\hline $\begin{array}{l}\text { Random sequence genera- } \\
\text { tion (selection bias) }\end{array}$ & Low risk & $\begin{array}{l}\text { Quote: "...they were randomly divided into } 4 \text { groups of } 24 \text { individuals by alpha- } \\
\text { betical order" }\end{array}$ \\
\hline $\begin{array}{l}\text { Allocation concealment } \\
\text { (selection bias) }\end{array}$ & Unclear risk & Not mentioned \\
\hline $\begin{array}{l}\text { Blinding of participants } \\
\text { and personnel (perfor- } \\
\text { mance bias) } \\
\text { All outcomes }\end{array}$ & Unclear risk & $\begin{array}{l}\text { Quote: "this was controlled, parallel, randomized one centre..." However, } \\
\text { method of blinding is not mentioned }\end{array}$ \\
\hline $\begin{array}{l}\text { Blinding of outcome as- } \\
\text { sessment (detection bias) } \\
\text { All outcomes }\end{array}$ & Unclear risk & $\begin{array}{l}\text { Quote: "this was controlled, parallel, randomized one centre..." However, } \\
\text { method of blinding is not mentioned }\end{array}$ \\
\hline $\begin{array}{l}\text { Incomplete outcome data } \\
\text { (attrition bias) } \\
\text { All outcomes }\end{array}$ & Low risk & Quote: "All 96 participants completed the study" \\
\hline $\begin{array}{l}\text { Selective reporting (re- } \\
\text { porting bias) }\end{array}$ & Low risk & $\begin{array}{l}\text { All outcomes described were reported. Conclusions are in accordance with the } \\
\text { results }\end{array}$ \\
\hline Other bias & Low risk & None \\
\hline
\end{tabular}

\section{Auschill 2007}

Title: a clinical comparison of 2 over-the-counter bleaching systems
Trial design: examiner-blinded, split-mouth randomised controlled trial
Location: Freiburg, Germany
Language: German (translates to English using Google translate)
Number of centres: 1
Recruitment period: not reported
Funding source: not reported

Participants

\author{
Participants: 21 to 36 years old. Mean age: 25.5 years \\ Total number: 26 \\ Inclusion criteria: \\ - A3 or darker shade \\ - presence of anterior teeth to premolar
}


Auschill 2007 (Continued)

Number randomised: 26

Method of randomisation: not reported

Method of allocation concealment: not reported

Method of blinding: not reported

Number evaluated: 20

\begin{tabular}{|c|c|}
\hline \multirow[t]{4}{*}{ Interventions } & Total number of intervention groups: 2 \\
\hline & $5.9 \%$ hydrogen peroxide gel \\
\hline & $5.9 \%$ hydrogen peroxide strips \\
\hline & Duration of treatment: 32 days \\
\hline \multirow[t]{2}{*}{ Outcomes } & Improvement in tooth shade \\
\hline & Vita shade guide: $\mathrm{C} 1$ lightest (1) to B4 darkest (16) \\
\hline \multirow[t]{5}{*}{ Notes } & Sample size calculation: not reported \\
\hline & Adverse effects: hypersensitivity \\
\hline & Health-related quality of life: not reported \\
\hline & $\begin{array}{l}\text { Key conclusions of the study authors: "The subjects teeth treated with the strips-system exhibited a } 6.0 \\
+/-0.0 \text { mean shade scores improvement compared to baseline ( } 53.7 \text { cycles; } 1610.3 \text { min), and the sub- } \\
\text { jects teeth treated with the gel-system exhibited a } 3.3+/-1.4 \text { mean shade scores improvement ( } 64.6 \\
\text { cycles; } 969.0 \text { min). However, both treatments were able to whiten teeth statistically significantly com- } \\
\text { pared to baseline. Side effects caused by the } 2 \text { systems were minimal and reversible. None of the teeth } \\
\text { studied showed detectible enamel surface changes in the subsequent SEM analysis. Both methods } \\
\text { were well accepted" }\end{array}$ \\
\hline & Contact: Thorsten.auschill@uniklini-freiburg.de \\
\hline
\end{tabular}

\section{Risk of bias}

\begin{tabular}{lll}
\hline Bias & Authors' judgement & Support for judgement \\
\hline $\begin{array}{l}\text { Random sequence genera- } \\
\text { tion (selection bias) }\end{array}$ & Unclear risk & $\begin{array}{l}\text { Quote:"...subjects were randomly assigned to one of two groups." However, } \\
\text { method is not reported }\end{array}$ \\
\hline $\begin{array}{l}\text { Allocation concealment } \\
\text { (selection bias) }\end{array}$ & Unclear risk & Not mentioned \\
\hline $\begin{array}{l}\text { Blinding of participants } \\
\text { and personnel (perfor- } \\
\text { mance bias) } \\
\text { All outcomes }\end{array}$ & Unclear risk & Not done \\
\hline
\end{tabular}

\begin{tabular}{lll}
\hline $\begin{array}{l}\text { Blinding of outcome as- } \\
\text { sessment (detection bias) } \\
\text { All outcomes }\end{array}$ & Unclear risk & $\begin{array}{l}\text { Quote: "Examiner-blinded split-mouth randomised controlled trial." However, } \\
\text { method is not reported }\end{array}$ \\
\hline $\begin{array}{l}\text { Incomplete outcome data } \\
\text { (attrition bias) } \\
\text { All outcomes }\end{array}$ & Low risk & Quote: "6 out of 26 patients no longer appeared for follow-up" \\
& $\begin{array}{l}\text { Comment: as each group had } 20 \text { participants (split-mouth trial) we rated it as } \\
\text { low risk according to Higgins } 2011 \text { Section } 8.5 . d . \text { Missing outcome data bal- } \\
\text { anced in numbers across intervention groups }\end{array}$ \\
\hline \hline
\end{tabular}


Auschill 2007 (Continued)

\begin{tabular}{|c|c|c|}
\hline $\begin{array}{l}\text { Selective reporting (re- } \\
\text { porting bias) }\end{array}$ & Low risk & $\begin{array}{l}\text { All outcomes described were reported. Conclusions are in accordance with the } \\
\text { results }\end{array}$ \\
\hline
\end{tabular}

Other bias Low risk None

Auschill 2012

Title: randomised clinical trial of the efficacy, tolerability, and long-term colour stability of 2 bleaching
techniques: 18-month follow-up
Trial design: 2-cell, parallel, examiner-blinded, randomised controlled trial
Location: Albert-Ludwigs-University, Freiburg, Germany
Language: English
Number of centres: 1
Recruitment period: not reported
Funding source: Colgate Palmolive

Participants

Participants: 18 to 56 years old, mean age: 33.08 years

Total number: 30

Inclusion criteria:

- maxillary central incisor with A3 or darker shade

- free of restorations

- no prior bleaching

Exclusion criteria:

- fixed orthodontic appliance

- tooth hypersensitivity

Number randomised: 30

Method of randomisation: a computer-based randomisation

Method of allocation concealment: not reported

Method of blinding: personal examiner blinding done by coding which was only known to statistician

Number evaluated: 28.2 dropouts at follow-up

Tray group: $5 \%$ hydrogen peroxide

Strip group: $5.3 \%$ hydrogen peroxide

Duration of treatment: 14 days

Vita shade guide: ranked from lightest B1 (1) to darkest C4 (16); mean score for anterior teeth was calculated

Adverse effects. Patient recorded: mild, moderate and severe 
Auschill 2012 (Continued)

Patient acceptance, gingival irritation, sensitivity

VAS scale: 0 (best acceptance) to 10 (no acceptance)

Sotes
Adverse effects: sensitivity
Key conclusions of the study authors: "Both bleaching techniques (the tray technique with 5.0\% hy-
drogen peroxide and the strip technique with 5.3\% hydrogen peroxide) demonstrated similar success.
Although a significant relapse in tooth shade was observed over an 18-month post bleaching period,
treated teeth were still significantly lighter compared to baseline. Adverse effects were minimal and re-
versible. Patient acceptance was statistically significantly higher in the tray group compared with the
strip group"
Correspondence required: no
Contact: Dr Thorsten M Auschill; auschill@med.uni-marburg.de

Risk of bias

\begin{tabular}{|c|c|c|}
\hline Bias & Authors' judgement & Support for judgement \\
\hline $\begin{array}{l}\text { Random sequence genera- } \\
\text { tion (selection bias) }\end{array}$ & Low risk & $\begin{array}{l}\text { Quote: "A computer-based randomisation scheme (generated before starting } \\
\text { the study) allocated patients..." }\end{array}$ \\
\hline $\begin{array}{l}\text { Allocation concealment } \\
\text { (selection bias) }\end{array}$ & Unclear risk & Not mentioned \\
\hline $\begin{array}{l}\text { Blinding of participants } \\
\text { and personnel (perfor- } \\
\text { mance bias) } \\
\text { All outcomes }\end{array}$ & Unclear risk & $\begin{array}{l}\text { Quote: "2-cell, parallel, examiner-blinded, randomised controlled trial." How- } \\
\text { ever, method is not mentioned }\end{array}$ \\
\hline $\begin{array}{l}\text { Blinding of outcome as- } \\
\text { sessment (detection bias) } \\
\text { All outcomes }\end{array}$ & Low risk & $\begin{array}{l}\text { Quote: "..examiners, who were blinded to the treatment modality and peri- } \\
\text { od, subjectively measured.. Personal blinding done by coding which was only } \\
\text { known to statistician" }\end{array}$ \\
\hline $\begin{array}{l}\text { Incomplete outcome data } \\
\text { (attrition bias) } \\
\text { All outcomes }\end{array}$ & Low risk & $\begin{array}{l}\text { Quote: "2 had to be classified as dropouts for that single visit. Thus, } 28 \text { sub- } \\
\text { jects were available at the } 18 \text {-month follow-up" } \\
\text { Comment: as each group had } 14 \text { participants each we considered it as low risk } \\
\text { according to Higgins } 2011 \text { Section } 8.5 . \text { d. Missing outcome data balanced in } \\
\text { numbers across intervention groups }\end{array}$ \\
\hline $\begin{array}{l}\text { Selective reporting (re- } \\
\text { porting bias) }\end{array}$ & Low risk & $\begin{array}{l}\text { All outcomes described were reported. Conclusions are in accordance with the } \\
\text { results }\end{array}$ \\
\hline Other bias & Low risk & None \\
\hline
\end{tabular}

Barlow 2003

Title: clinical response of 2 brush-applied peroxide whitening systems
Trial design: double-blinded, randomised, controlled, parallel-group trial
Location: university
Language: English


Barlow 2003 (Continued)

Number of centres: 1

Recruitment period: not reported

Funding source: Colgate Palmolive

Participants: 18 to 48 years old, mean age: 23 years
Total number: 38
Inclusion criteria:
- healthy adults who provided informed consent
Exclusion criteria:
- poor general and oral health
- prior bleaching
- patients with orthodontic appliances and restorations in their maxillary anterior teeth
Number randomised: 38
Method of randomisation: not reported
Method of allocation concealment: not reported
Method of blinding: similar packet kits
Number evaluated: 38

Interventions

Total number of intervention groups: 2

$18 \%$ carbamide peroxide

$19 \%$ sodium percarbonate ( 2 brush-applied whitening kits)

Duration of treatment: 14 days

Outcomes

Improvement in tooth colour

$b^{\star}$ : decreased $b^{\star}$ indicates reduced yellowness

$\Delta \mathrm{L}$ : increased $\Delta \mathrm{L}$ is increased brightness

$\Delta W$ : negative $\Delta W$ indicates colour closer to white

Notes Sample size calculation: not reported

Adverse effects: sensitivity and gingival irritation

Health-related quality of life: not reported

Key conclusions of the study authors: "Crest Night Effect provided significant and meaningful improvement in colour after 14 days... Both products were tolerated"

Correspondence required: no

Contact: A Barlow; barlow.ap@pg.com

\section{Risk of bias}


Barlow 2003 (Continued)

Random sequence genera- Unclear risk Quote:"double-blind, randomised, controlled, parallel-group clinical trial." tion (selection bias)

However, method of randomisation not reported

\begin{tabular}{lll}
\hline $\begin{array}{l}\text { Allocation concealment } \\
\text { (selection bias) }\end{array}$ & Unclear risk & Not mentioned \\
\hline $\begin{array}{l}\text { Blinding of participants } \\
\text { and personnel (perfor- } \\
\text { mance bias) } \\
\text { All outcomes }\end{array}$ & Low risk & Quote: "Participants were supplied with blinded study kit boxes" \\
\hline $\begin{array}{l}\text { Blinding of outcome as- } \\
\text { sessment (detection bias) } \\
\text { All outcomes }\end{array}$ & Unclear risk & $\begin{array}{l}\text { Quote: "double-blind, randomised, controlled, parallel-group clinical trial." } \\
\text { However, method of blinding not reported }\end{array}$ \\
\hline $\begin{array}{l}\text { Incomplete outcome data } \\
\begin{array}{l}\text { (attrition bias) } \\
\text { All outcomes }\end{array}\end{array}$ & Low risk & No dropouts reported \\
\hline $\begin{array}{l}\text { Selective reporting (re- } \\
\text { porting bias) }\end{array}$ & Low risk & \\
\hline \begin{tabular}{l} 
Other bias \\
\hline
\end{tabular} & Low risk & $\begin{array}{l}\text { All outcomes described were reported. Conclusions are in accordance with the } \\
\text { results }\end{array}$ \\
\hline
\end{tabular}

\section{Berga-Caballero 2006}

Methods

Title: at-home vital bleaching: a comparison of hydrogen peroxide and carbamide peroxide treatments

Trial design: randomised controlled trial

Location: not reported

Language: English

Number of centres: 1

Recruitment period: not reported

Funding source: not reported

\begin{tabular}{ll}
\hline Participants & Participants: age not reported \\
Total number: 6 \\
Inclusion criteria: not reported \\
Exclusion criteria: not reported \\
Number randomised: 6 \\
Method of randomisation: not reported \\
Method of allocation concealment: not reported \\
Method of blinding: not reported \\
Number evaluated: 6
\end{tabular}

Interventions

Total number of intervention groups: 2 
Berga-Caballero 2006 (Continued)

$10 \%$ carbamide peroxide: 20 to 28 days

$3.5 \%$ hydrogen peroxide: 28 days

Duration of treatment: 20 to 28 days

\begin{tabular}{ll}
\hline Outcomes & Improvement in tooth shade \\
\hline Notes & Sample size calculation: not reported \\
Adverse effects: sensitivity \\
Health-related quality of life: not reported \\
Key conclusions of the study authors: "In carbamide peroxide group, the daily length of application was \\
2 hours. In the first case the treatment lasted for 24 days, there was no pre- or post-operative sensitivi- \\
ty and the tooth shade changed from A4 (canines) - A3.5 (incisors) to A2 (canines) - A1 (incisors). In hy- \\
drogen peroxide the duration of treatment was similar (28 days), no sensitivity was shown during treat- \\
ment and the shade changed from A3.5 (canines) - A3 (incisors) to A1. Case 3 presented a change in \\
shade from A4 (canines) - A3 (incisors) to A2 (canines) - A1 (incisors) after only 20 days; in this case the \\
patient did mention slight sensitivity in the anterior mandibular teeth throughout the treatment" \\
Correspondence required: no \\
Contact: Professor Dr Forner Navarro; forner@uv.es \\
\end{tabular}

\section{Risk of bias}

\begin{tabular}{lll}
\hline Bias & Authors' judgement & Support for judgement \\
\hline $\begin{array}{l}\text { Random sequence genera- } \\
\text { tion (selection bias) }\end{array}$ & Unclear risk & $\begin{array}{l}\text { Quote: "They were randomly assigned to the two treatment groups." However, } \\
\text { the method is not mentioned }\end{array}$ \\
\hline $\begin{array}{l}\text { Allocation concealment } \\
\text { (selection bias) }\end{array}$ & Unclear risk & Not mentioned \\
\hline $\begin{array}{l}\text { Blinding of participants } \\
\text { and personnel (perfor- } \\
\text { mance bias) } \\
\text { All outcomes }\end{array}$ & Unclear risk & Not mentioned \\
\hline $\begin{array}{l}\text { Blinding of outcome as- } \\
\text { sessment (detection bias) } \\
\text { All outcomes }\end{array}$ & Unclear risk & Not mentioned \\
\hline $\begin{array}{l}\text { Incomplete outcome data } \\
\text { (attrition bias) }\end{array}$ & Low risk & All subjects completed the trial \\
\hline $\begin{array}{l}\text { Selective reporting (re- } \\
\text { porting bias) }\end{array}$ & Low risk & Low risk \\
\hline $\begin{array}{l}\text { Other bias } \\
\text { results }\end{array}$ & None \\
\hline
\end{tabular}

\section{Biesbrock 2004}

\section{Methods}

Title: a chewing gum containing $7.5 \%$ sodium hexametaphosphate inhibits stain deposition compared with a placebo chewing gum 
Biesbrock 2004 (Continued)

Trial design: cross-over randomised controlled trial

Location: USA

Language: English

Number of centres: 1

Recruitment period: not reported

Funding source: Procter \& Gamble

Participants: 18 to 70 years old. Mean age: 39.1 years
Total number: 20
Inclusion criteria:
- 16 natural teeth with minimum 7 anterior teeth
Exclusion criteria:
- previous history of hypersensitivity to test products
- multiple restorations
- fixed prosthesis
Number randomised: 19
Method of randomisation: not reported
Method of allocation concealment: not reported
Method of blinding: not reported
Number evaluated: 18

Interventions

Total number of intervention groups: 2 (cross-over)

$7.5 \%$ sodium hexametaphosphate chewing gum

Placebo

Duration of treatment: 2 weeks with 78 hours washout time

\begin{tabular}{ll}
\hline Outcomes & Improvement in tooth shade: reduction in stain \\
$\Delta \mathrm{L}, \mathrm{a}^{\star}, \mathrm{b}^{\star}$ values were recorded. Increase in $\mathrm{L}$ and reduction in b indicated whitening \\
\hline Sotes & Sample size calculation: not reported \\
& Adverse effects: not reported \\
& Health-related quality of life: not reported \\
& Key conclusions of the study authors: "Sodium hexametaphosphate delivered from a chewing gum pre- \\
vents dental stain formation and facilitates stain removal, which leads to a perceptible whitening bene- \\
fit" \\
Correspondence required: no \\
Contact: Dr Aron RB, Procter and Gamble Company, Healthcare Research Center, Manson, Ohio, USA
\end{tabular}

\section{Risk of bias}


Biesbrock 2004 (Continued)

\begin{tabular}{|c|c|c|}
\hline Bias & Authors' judgement & Support for judgement \\
\hline $\begin{array}{l}\text { Random sequence genera- } \\
\text { tion (selection bias) }\end{array}$ & Unclear risk & $\begin{array}{l}\text { Quote: "Subjects were randomly assigned to one of two treatment groups." } \\
\text { However, method was not reported }\end{array}$ \\
\hline $\begin{array}{l}\text { Allocation concealment } \\
\text { (selection bias) }\end{array}$ & Unclear risk & Not mentioned \\
\hline $\begin{array}{l}\text { Blinding of participants } \\
\text { and personnel (perfor- } \\
\text { mance bias) } \\
\text { All outcomes }\end{array}$ & Unclear risk & $\begin{array}{l}\text { Quote: "Randomised, double-blinded cross-over trial." However, method was } \\
\text { not reported }\end{array}$ \\
\hline $\begin{array}{l}\text { Blinding of outcome as- } \\
\text { sessment (detection bias) } \\
\text { All outcomes }\end{array}$ & Unclear risk & $\begin{array}{l}\text { Quote: "Randomised, double-blinded cross-over trial." However, method was } \\
\text { not reported }\end{array}$ \\
\hline $\begin{array}{l}\text { Incomplete outcome data } \\
\text { (attrition bias) } \\
\text { All outcomes }\end{array}$ & Low risk & $\begin{array}{l}\text { Quote: "1 woman abandoned the study" } \\
\text { Comment: plausible effect size (difference in means) among missing outcomes } \\
\text { not enough to have a clinically relevant impact on observed effect size }\end{array}$ \\
\hline $\begin{array}{l}\text { Selective reporting (re- } \\
\text { porting bias) }\end{array}$ & Low risk & $\begin{array}{l}\text { All outcomes described were reported. Conclusions are in accordance with the } \\
\text { results }\end{array}$ \\
\hline Other bias & Low risk & None \\
\hline
\end{tabular}

\section{Bizhang 2007}

Title: clinical trial of long-term colour stability of hydrogen peroxide strips and sodium percarbonate
film
Trial design: randomised, placebo-controlled trial
Location: Berlin, Germany
Language: English
Number of centres: 1
Recruitment period: not reported
Funding source: not reported

Participants

Participants: 18 to 60 years old, mean age: 30 years

Total number: 72

Inclusion criteria:

- healthy adults with at least 16 natural teeth including 4 maxillary incisors

- tooth shade score of A2

Exclusion criteria:

- prior bleaching history

- current sensitivity

- acute dental treatment needs 
Bizhang 2007 (Continued)

Number randomised: 72

Method of randomisation: not reported

Method of allocation concealment: not reported

Method of blinding: blinded test kits

Number evaluated: 70

\begin{tabular}{|c|c|}
\hline \multirow[t]{5}{*}{ Interventions } & Total number of intervention groups: 3 \\
\hline & $6 \%$ hydrogen peroxide whitening strips \\
\hline & $19 \%$ sodium percarbonate brush-applied gel that dries to a film \\
\hline & Placebo brush-applied gel without peroxide \\
\hline & Duration of treatment: 2 weeks \\
\hline \multirow[t]{2}{*}{ Outcomes } & Improvement in tooth shade \\
\hline & $\begin{array}{l}\text { Tooth whitening was characterized by decreased } b^{\star} \text { (reduction in yellowness) and increased } \Delta L^{\star} \text { (in- } \\
\text { creased brightness) }\end{array}$ \\
\hline \multirow[t]{6}{*}{ Notes } & Sample size calculation: not reported \\
\hline & Adverse effects: tooth sensitivity and oral irritation \\
\hline & Health-related quality of life: not reported \\
\hline & $\begin{array}{l}\text { Key conclusions of the study authors: "6\% hydrogen peroxide whitening strips yielded significant ( } P \\
<0.02 \text { ) initial whitening relative to baseline, placebo and a } 19 \% \text { sodium percarbonate, brush-applied } \\
\text { film. All treatments were well-tolerated, both peroxide-containing systems exhibited appreciable col- } \\
\text { or retention throughout the } 18 \text {-month post-treatment period, and here were no meaningful, persistent } \\
\text { adverse events seen with long-term follow-up" }\end{array}$ \\
\hline & Correspondence required: no \\
\hline & Contact: Dr Mozhgan Bizhang; mozhgan.bizhang@med.uni-duesseldorf.de \\
\hline
\end{tabular}

\section{Risk of bias}

\begin{tabular}{|c|c|c|}
\hline Bias & Authors' judgement & Support for judgement \\
\hline $\begin{array}{l}\text { Random sequence genera- } \\
\text { tion (selection bias) }\end{array}$ & Unclear risk & $\begin{array}{l}\text { Quote: "A total of } 72 \text { subjects from the greater Berlin metropolitan area were } \\
\text { randomised equally to the strip, film and placebo groups." However, the } \\
\text { method is not mentioned }\end{array}$ \\
\hline $\begin{array}{l}\text { Allocation concealment } \\
\text { (selection bias) }\end{array}$ & Unclear risk & Not mentioned \\
\hline $\begin{array}{l}\text { Blinding of participants } \\
\text { and personnel (perfor- } \\
\text { mance bias) } \\
\text { All outcomes }\end{array}$ & Low risk & Quote: "Products were dispensed in a blinded subject kit box" \\
\hline $\begin{array}{l}\text { Blinding of outcome as- } \\
\text { sessment (detection bias) } \\
\text { All outcomes }\end{array}$ & Low risk & $\begin{array}{l}\text { Quote: "With the examiners still blinded as to treatment, these subjects (strip } \\
\text { and film groups) were evaluated at post-treatment months } 12,15,16 \text {, and } 18 \text { " }\end{array}$ \\
\hline
\end{tabular}


Bizhang 2007 (Continued)

Incomplete outcome data Low risk Quote: "With respect to subject disposition, 71 subjects completed the end-of(attrition bias) treatment visit, and $70(97 \%)$ completed the month 6 ..."

All outcomes

Comment: 2 dropouts were noted. Plausible effect size (difference in means) among missing outcomes not enough to have a clinically relevant impact on observed effect size

\begin{tabular}{lll}
\hline $\begin{array}{l}\text { Selective reporting (re- } \\
\text { porting bias) }\end{array}$ & Low risk & $\begin{array}{l}\text { All outcomes described were reported. Conclusions are in accordance with the } \\
\text { results }\end{array}$ \\
\hline Other bias & Low risk & None \\
\hline
\end{tabular}

\section{Botelho 2017}

\section{Methods}

Title: a randomised controlled trial of home bleaching of tetracycline-stained teeth

Trial design: randomised, examiner-blinded controlled trial

Location: University of Hong Kong

Language: English

Number of centres: 1

Recruitment period: not reported

Funding source: not reported

\section{Participants}

Participants: tray group: mean age 28.7 years; strip group: mean age 30.4 years

Total number: 36

Inclusion criteria:

- tetracycline stained teeth

- maxillary anterior teeth sound or minimally restored

- able to attend 4-month review

Exclusion criteria:

- subjects under 18

- medically unfit

- pregnant or lactating

- uncontrolled oral disease or infection

- history of tooth whitening treatment

- smoker

- allergy to hydrogen peroxide or carbamide peroxide

Number randomised: 26

Method of randomisation: coin toss

Method of allocation concealment: not reported

Method of blinding: not reported

Number evaluated: 24 ( 2 dropouts (1 for each group) at follow-up) 
Botelho 2017 (Continued)

Tray: 15\% carbamide peroxide

Strip: $6.5 \%$ hydrogen peroxide

Duration of treatment: 3 months

\begin{tabular}{|c|c|}
\hline Outcomes & $\begin{array}{l}\text { Improvement in tooth colour } \\
a^{\star}, b^{\star} \text { and } \Delta L \text { were recorded } \\
\text { Whitening benefit was represented by negative } b^{\star} \text { (yellowness reduction), and positive } \Delta L \text { (increasing } \\
\text { lightness) }\end{array}$ \\
\hline Notes & $\begin{array}{l}\text { Sample size calculation: done } \\
\text { Adverse effects: sensitivity } \\
\text { Health-related quality of life: not reported } \\
\text { Key conclusions of the study authors: "Both groups experienced noticeable and significant } \Delta \mathrm{L}^{\star} \mathrm{a}^{\star} \mathrm{b}^{\star} \text { im- } \\
\text { provement at the end of the trial in comparison to the baseline. Significant improvement was observed } \\
\text { in the first month for the tray group and in the first } 2 \text { months for the strip group }(\mathrm{P}<0.05) \text {. While greater } \\
\text { lightness improvement was observed in the tray group over the strip group in the first month, the op- } \\
\text { posite was noticed in the second month. There was no difference between } 2 \text { groups at the end of this } \\
\text { trial and no adverse reactions were observed" } \\
\text { Correspondence required: no } \\
\text { Contact: Dr Botelho MG; botelho@hku.hk }\end{array}$ \\
\hline
\end{tabular}

\section{Risk of bias}

\begin{tabular}{|c|c|c|}
\hline Bias & Authors' judgement & Support for judgement \\
\hline $\begin{array}{l}\text { Random sequence genera- } \\
\text { tion (selection bias) }\end{array}$ & Low risk & Quote: "Participants were randomly assigned to either group..... tossing coin" \\
\hline $\begin{array}{l}\text { Allocation concealment } \\
\text { (selection bias) }\end{array}$ & Unclear risk & Not mentioned \\
\hline $\begin{array}{l}\text { Blinding of participants } \\
\text { and personnel (perfor- } \\
\text { mance bias) } \\
\text { All outcomes }\end{array}$ & Unclear risk & $\begin{array}{l}\text { Quote: "Participants were clinically review by one reviewer who was blinded to } \\
\text { their treatment." However, method of blinding is not reported }\end{array}$ \\
\hline $\begin{array}{l}\text { Blinding of outcome as- } \\
\text { sessment (detection bias) } \\
\text { All outcomes }\end{array}$ & Unclear risk & $\begin{array}{l}\text { Quote: "Participants were clinically review by one reviewer who was blinded to } \\
\text { their treatment." However, method of blinding is not reported }\end{array}$ \\
\hline $\begin{array}{l}\text { Incomplete outcome data } \\
\text { (attrition bias) } \\
\text { All outcomes }\end{array}$ & Low risk & $\begin{array}{l}\text { Quote: "..each group had } 1 \text { participant that did not attend } 2 \text { months review" } \\
\text { Comment: missing outcome data balanced in numbers across intervention } \\
\text { groups }\end{array}$ \\
\hline $\begin{array}{l}\text { Selective reporting (re- } \\
\text { porting bias) }\end{array}$ & Low risk & $\begin{array}{l}\text { All outcomes described were reported. Conclusions are in accordance with the } \\
\text { results }\end{array}$ \\
\hline Other bias & Low risk & None \\
\hline
\end{tabular}


Browning 2008

Methods
Title: comparison of traditional and low sensitivity whiteners

Trial design: double-blinded, placebo-controlled clinical trial

Location: Department of Restorative Dentistry, Indiana University School of Dentistry, USA

Language: English

Number of centres: 1

Recruitment period: not reported

Funding source: not reported

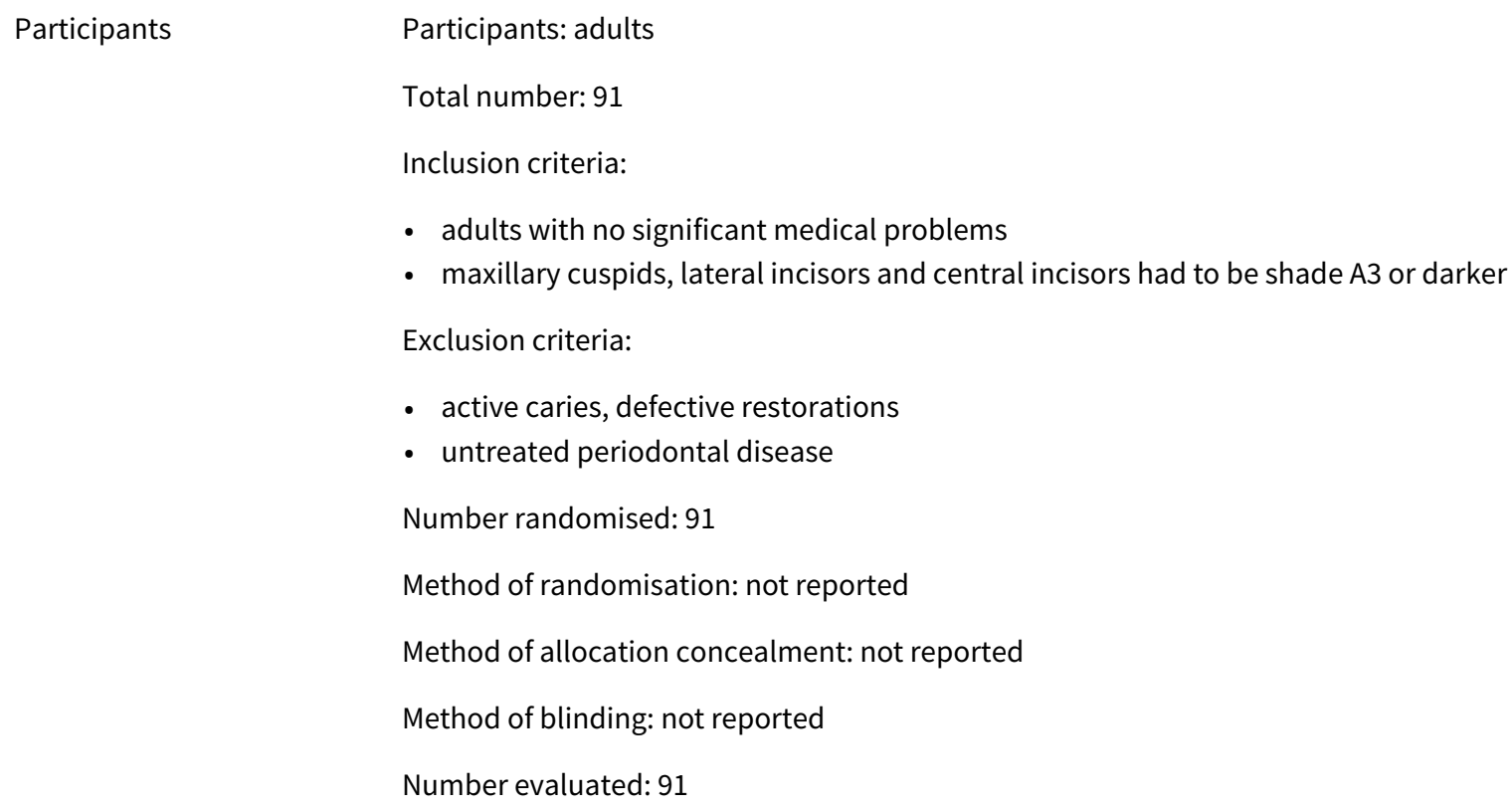
um nitrate had sensitivity levels equivalent to those using the placebo. Relative to the whitening agent with no desensitising agent, the addition of $0.5 \%$ potassium nitrate resulted in a significant reduction in the number of days of sensitivity experienced by participants. When compared to the whitener without any potassium nitrate, the addition of $3 \%$ potassium nitrate did not result in a significant reduction 
Browning 2008 (Continued)

in the number of days of sensitivity. The addition of potassium nitrate did not result in any significant change in bleaching efficacy"

Correspondence required: no

Contact: William D Browning; lewis46@iupui.edu

\section{Risk of bias}

\begin{tabular}{|c|c|c|}
\hline Bias & Authors' judgement & Support for judgement \\
\hline $\begin{array}{l}\text { Random sequence genera- } \\
\text { tion (selection bias) }\end{array}$ & Unclear risk & $\begin{array}{l}\text { Quote: "placebo-controlled, double-blind randomised clinical trial com- } \\
\text { pared..." However, method not mentioned }\end{array}$ \\
\hline $\begin{array}{l}\text { Allocation concealment } \\
\text { (selection bias) }\end{array}$ & Unclear risk & Method not mentioned \\
\hline $\begin{array}{l}\text { Blinding of participants } \\
\text { and personnel (perfor- } \\
\text { mance bias) } \\
\text { All outcomes }\end{array}$ & Unclear risk & $\begin{array}{l}\text { Quote: "Neither the participant nor the operator was aware which material } \\
\text { was received." However, method is not mentioned }\end{array}$ \\
\hline $\begin{array}{l}\text { Blinding of outcome as- } \\
\text { sessment (detection bias) } \\
\text { All outcomes }\end{array}$ & Unclear risk & $\begin{array}{l}\text { Quote: "Neither the participant nor the operator was aware which material } \\
\text { was received." However, method is not mentioned }\end{array}$ \\
\hline $\begin{array}{l}\text { Incomplete outcome data } \\
\text { (attrition bias) } \\
\text { All outcomes }\end{array}$ & Low risk & No dropouts are mentioned \\
\hline $\begin{array}{l}\text { Selective reporting (re- } \\
\text { porting bias) }\end{array}$ & Low risk & $\begin{array}{l}\text { All outcomes described were reported. Conclusions are in accordance with the } \\
\text { results }\end{array}$ \\
\hline Other bias & Low risk & None \\
\hline
\end{tabular}

\section{Bruhn 2012}

\section{Methods}

Title: vital tooth whitening effects on oral health-related quality of life in older adults

Trial design: single-blinded, randomised, pre-test, multiple post-test design

Location: Hampton Roads area of Virginia, USA

Language: English

Number of centres: 1

Recruitment period: not reported

Funding source: not reported

\section{Participants}

\section{Participants: 50 years old and above}

Total number: 62

Inclusion criteria:

- 50 years old and older

- good general health, possessing cognitive ability 
- physical dexterity to perform daily oral care

- have at least 8 natural anterior teeth free from composite restorations, crowns, veneers

- no full or partial dentures and endodontic treatment

- refrain from using any over-the-counter tooth whitening products for the duration of the study

Exclusion criteria:

- visible calculus deposits on labial or lingual surfaces of anterior teeth

- severe tooth sensitivity

- professional whitening within the past 3 years

Number randomised: 62

Method of randomisation: not reported

Method of allocation concealment: not reported

Method of blinding: participants were assigned a number after being randomly assigned to a group

Number evaluated: 53

Interventions

Total number of intervention groups: 2

Control: placebo

Experimental: $14 \%$ hydrogen peroxide

Duration of treatment: 3 weeks

Outcomes Trubyte New Hue Vitality Scale: 12 shades numbered from 1 to 12 , with 1 being the lightest and 12 being the darkest

Tooth Colour Satisfaction Scale (TCSS): very satisfied (5 points), satisfied (4 points), neither satisfied nor dissatisfied ( 3 points), dissatisfied ( 2 points) and very dissatisfied (1 point)

Oral Health Impact Profile (OHIP): 5-point Likert scale: very often (5 points), fairly often (4 points), occasionally (3 points), hardly ever ( 2 points), never (1 point)

Additional Questions Survey (AQS): none (1 point), 1 to 2 ( 2 points), 3 to 4 (3 points), 5 to 6 (4 points) and 7 or more (5 points)

Notes

Sample size calculation: not reported

Adverse effects: sensitivity

Health-related quality of life: reported

Key conclusions of the study authors: "The older adults who whitened their teeth experienced an increased satisfaction with their tooth colour as evidenced by the TCSS. Tooth whitening was not associated with improvements in overall OHRQoL, or its functional factors, psychological disabilities, psychological discomforts, physical disabilities and social disabilities subscales. Tooth whitening did affect the handicap subscale, which demonstrated that persons who experienced tooth whitening were more willing to work due to a perceived increase in health. Tooth whitening did affect the physical pain subscale, which demonstrated a lower OHRQoL for participants. Older adults who whitened their teeth reported fewer social activities 3 months after the initial post-testing. Regression analysis relating tooth colour satisfaction with overall OHRQoL revealed a significant correlation between tooth colour satisfaction and overall OHIP for the experimental group"

Correspondence required: no

Contact: Ann M Bruhn; abruhn@odu.edu 
Bruhn 2012 (Continued)

Risk of bias

\begin{tabular}{|c|c|c|}
\hline Bias & Authors' judgement & Support for judgement \\
\hline $\begin{array}{l}\text { Random sequence genera- } \\
\text { tion (selection bias) }\end{array}$ & Unclear risk & $\begin{array}{l}\text { Quote: " } 62 \text { participants } 50 \text { years of age and older were enrolled and randomly } \\
\text { assigned to } 1 \text { of } 2 \text { groups by research assistants." However, the method is not } \\
\text { mentioned }\end{array}$ \\
\hline $\begin{array}{l}\text { Allocation concealment } \\
\text { (selection bias) }\end{array}$ & Unclear risk & Not reported \\
\hline $\begin{array}{l}\text { Blinding of participants } \\
\text { and personnel (perfor- } \\
\text { mance bias) } \\
\text { All outcomes }\end{array}$ & Unclear risk & Not mentioned \\
\hline $\begin{array}{l}\text { Blinding of outcome as- } \\
\text { sessment (detection bias) } \\
\text { All outcomes }\end{array}$ & Low risk & $\begin{array}{l}\text { Quote: "Clinicians collecting data were unaware of participant group status, } \\
\text { since the participants were assigned a number after being randomly assigned } \\
\text { to" }\end{array}$ \\
\hline $\begin{array}{l}\text { Incomplete outcome data } \\
\text { (attrition bias) } \\
\text { All outcomes }\end{array}$ & Unclear risk & $\begin{array}{l}\text { Quote: "A total of } 53 \text { participants completed the study" } \\
\text { Comment: } 9 \text { dropouts were noted. Reason for dropouts not mentioned }\end{array}$ \\
\hline $\begin{array}{l}\text { Selective reporting (re- } \\
\text { porting bias) }\end{array}$ & Low risk & $\begin{array}{l}\text { All outcomes described were reported. Conclusions are in accordance with the } \\
\text { results }\end{array}$ \\
\hline Other bias & Low risk & None \\
\hline
\end{tabular}

Brunton 2004

Title: a 6-month study of 2 self-applied tooth whitening products containing carbamide peroxide
Trial design: double-blinded, randomised, controlled, parallel-group clinical trial
Location: not reported
Language: English
Number of centres: not reported
Recruitment period: not reported
Funding source: Colgate Palmolive
Participants: 18 to 70 years old
Total number: 95
Inclusion criteria:
Participants
- male and female subjects ranging in age from 18 to 70 years inclusive
- all maxillary anterior teeth present
- availability for the 6-month duration of the study
- a minimum Vita shade of A3 on 1 or more upper central incisors
Exclusion criteria:


- presence of orthodontic appliances or any anterior tooth with a prosthetic crown or veneer

- tumours or significant pathology of the soft or hard tissues of the oral cavity

- moderate or advanced periodontal disease, rampant caries or any condition that the dental examiner considered exclusionary from the study

- 5 or more carious lesions requiring immediate care

- participation in any other study within 30 days preceding the clinical study

- pregnant or lactating females

- a history of allergies to tooth whitening products, personal care consumer products or their ingredients

- restorations on the teeth to be scored

Number randomised: 95

Method of randomisation: not reported

Method of allocation concealment: not reported

Method of blinding: not reported

Number evaluated: 93, 2 dropouts

Interventions

Total number of intervention groups: 2

18\% (Group 1) carbamide peroxide

16.4\% (Group 2) carbamide peroxide

Duration of treatment: 2 weeks

Outcomes Improvement in tooth shade

The shade guide was arranged with the 16 -shade tabs in order from B1 (1) to C4 (16)

Gingival score: Loë and Silness Gingival Index

Gingival and teeth sensitivity score: 0 none -5 severe

Sample size calculation: not reported
Adverse effects: sensitivity
Health-related quality of life: not reported
Key conclusions of the study authors: "Both products effectively whitened teeth with a treatment time
of 2-weeks. The different concentrations tested were equally effective in improving whiteness. The
whitening systems tested produced little tooth or gingival sensitivity. Some whitening benefit is sus-
tained for at least 6 months after cessation of treatment"
Correspondence required: no
Contact: Paul A Brunton; paul.brunton@man.ac.uk

\section{Risk of bias}

\begin{tabular}{lll}
\hline Bias & Authors' judgement & Support for judgement \\
\hline $\begin{array}{l}\text { Random sequence genera- } \\
\text { tion (selection bias) }\end{array}$ & Unclear risk & $\begin{array}{l}\text { Quote: "double-blinded, randomised, controlled, parallel-group clinical trial." } \\
\text { However, method of randomisation not reported }\end{array}$ \\
\hline $\begin{array}{l}\text { Allocation concealment } \\
\text { (selection bias) }\end{array}$ & Unclear risk & Not mentioned \\
\hline
\end{tabular}


Brunton 2004 (Continued)

Blinding of participants Unclear risk Quote: "double-blinded, randomised, controlled, parallel-group clinical trial." and personnel (perforHowever, method of blinding not reported mance bias)

All outcomes

\begin{tabular}{|c|c|c|}
\hline $\begin{array}{l}\text { Blinding of outcome as- } \\
\text { sessment (detection bias) }\end{array}$ & Unclear risk & $\begin{array}{l}\text { Quote: "double-blinded, randomised, controlled, parallel-group clinical trial." } \\
\text { However, method of blinding not reported }\end{array}$ \\
\hline
\end{tabular}

All outcomes

Incomplete outcome data Low risk (attrition bias)

All outcomes

\begin{tabular}{lll}
\hline $\begin{array}{l}\text { Selective reporting (re- } \\
\text { porting bias) }\end{array}$ & Low risk & $\begin{array}{l}\text { All outcomes described were reported. Conclusions are in accordance with the } \\
\text { results }\end{array}$ \\
\hline Other bias & Low risk & None \\
\hline
\end{tabular}

Quote: "2 subjects failed to complete the 2-week study (1 from each group) for reasons unrelated to the study" Comment: missing outcome data balanced in numbers across intervention groups

\section{Cibirka 1999}

\begin{tabular}{|c|c|}
\hline \multirow[t]{7}{*}{ Methods } & $\begin{array}{l}\text { Title: clinical study of tooth shade lightening from dentist-supervised, patient-applied treatment with } 2 \\
10 \% \text { carbamide peroxide gels }\end{array}$ \\
\hline & Trial design: randomised double-blinded study \\
\hline & Location: not reported \\
\hline & Language: English \\
\hline & Number of centres: 1 \\
\hline & Recruitment period: not reported \\
\hline & Funding source: Ultradent \\
\hline \multirow[t]{13}{*}{ Participants } & Participants: 18 years old and above \\
\hline & Total number: not reported \\
\hline & Inclusion criteria: not reported \\
\hline & Exclusion criteria: \\
\hline & - persons with significant medical problems \\
\hline & - pregnant or nursing women \\
\hline & - persons with a history of vital bleaching \\
\hline & - ongoing fixed orthodontic treatment \\
\hline & Number randomised: not reported \\
\hline & Method of randomisation: randomisation table \\
\hline & Method of allocation concealment: not mentioned \\
\hline & Method of blinding: not reported \\
\hline & Number evaluated: 66 \\
\hline
\end{tabular}


Cibirka 1999 (Continued)

$10 \%$ carbamide peroxide from 2 different brands

Duration of treatment: 2 weeks

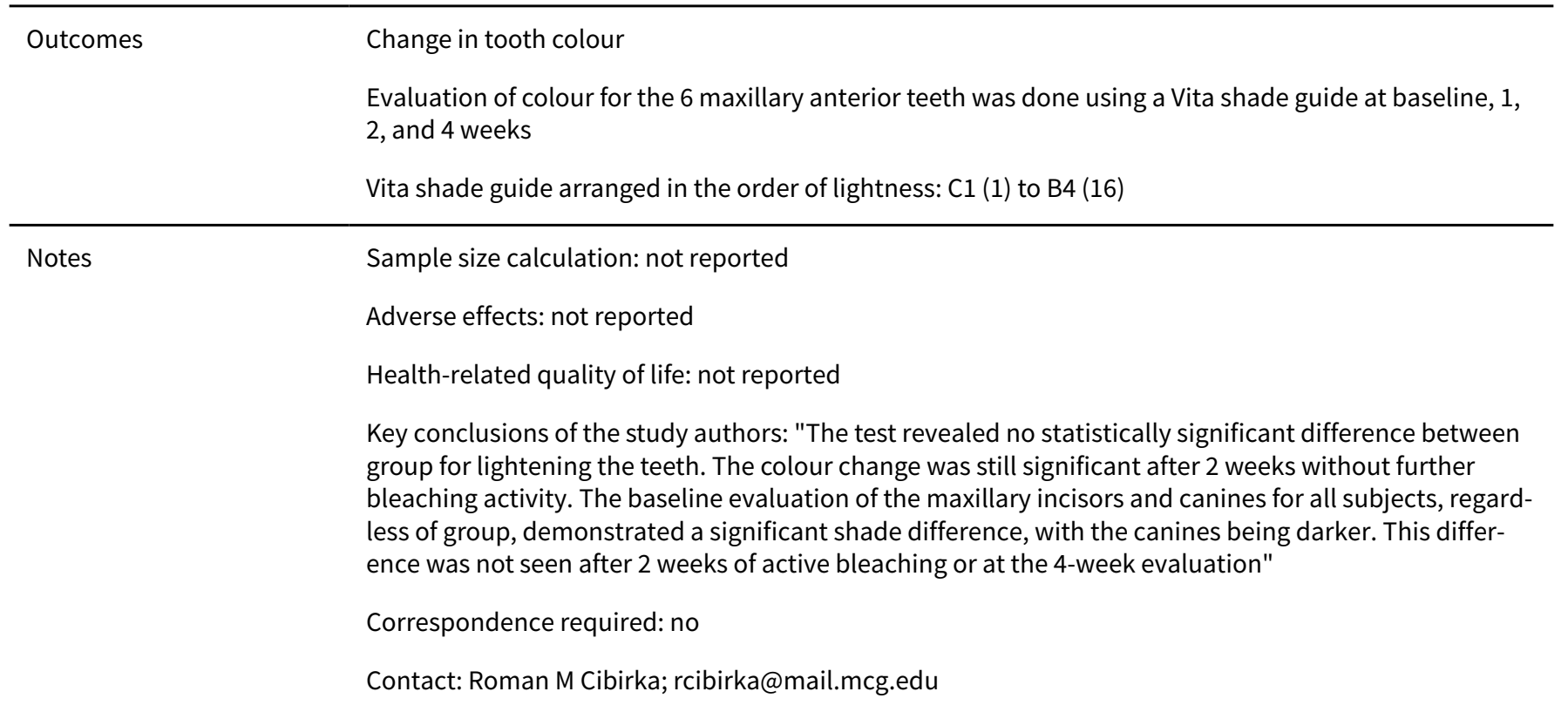

\section{Risk of bias}

\begin{tabular}{|c|c|c|}
\hline Bias & Authors' judgement & Support for judgement \\
\hline $\begin{array}{l}\text { Random sequence genera- } \\
\text { tion (selection bias) }\end{array}$ & Low risk & $\begin{array}{l}\text { Quote: "A statistician created a randomization table for all subjects and main- } \\
\text { tained subject identification" }\end{array}$ \\
\hline $\begin{array}{l}\text { Allocation concealment } \\
\text { (selection bias) }\end{array}$ & Unclear risk & Not mentioned \\
\hline $\begin{array}{l}\text { Blinding of participants } \\
\text { and personnel (perfor- } \\
\text { mance bias) } \\
\text { All outcomes }\end{array}$ & Low risk & $\begin{array}{l}\text { Quote: "..outside of the box and the individual syringes were labelled with the } \\
\text { subject number" }\end{array}$ \\
\hline $\begin{array}{l}\text { Blinding of outcome as- } \\
\text { sessment (detection bias) } \\
\text { All outcomes }\end{array}$ & Unclear risk & $\begin{array}{l}\text { Quote: "randomised double-blinded study." However, the method is not men- } \\
\text { tioned }\end{array}$ \\
\hline $\begin{array}{l}\text { Incomplete outcome data } \\
\text { (attrition bias) } \\
\text { All outcomes }\end{array}$ & Unclear risk & $\begin{array}{l}\text { The number of subjects randomised to both groups ( } n=32 \text { in both groups) ex- } \\
\text { plained in the methodology does not match the numbers shown in the table ( } n \\
=32 \text { and } n=34 \text { ) }\end{array}$ \\
\hline $\begin{array}{l}\text { Selective reporting (re- } \\
\text { porting bias) }\end{array}$ & Low risk & $\begin{array}{l}\text { All outcomes described were reported. Conclusions are in accordance with the } \\
\text { results }\end{array}$ \\
\hline Other bias & Low risk & None \\
\hline
\end{tabular}




\section{Methods}

Title: clinical evaluation of a novel whitening gel, containing $6 \%$ hydrogen peroxide and a standard fluoride toothpaste

Trial design: examiner-blinded, stratified, parallel-design clinical trial

Location: not reported

Language: English

Number of centres: 1

Recruitment period: not reported

Participants

Participants: 18 to 63 years old. Mean age 39.45 years

Total number: 128

Inclusion criteria:

- in good general health and had a desire to lighten their upper and lower anterior teeth

- pregnant or lactating women

- subjects were required to have at least 4 upper anterior teeth and 4 lower incisors

Exclusion criteria:

- orthodontic bands

- self-reported tooth hypersensitivity

- crowns/veneers or visible cosmetic restorations

- subjects with advanced periodontal disease

- used mouthrinses for the treatment/control of a periodontal condition

Number randomised: 128

Method of randomisation: stratified randomisation

Method of allocation concealment: not reported

Method of blinding: not reported

Number evaluated: 117
Total number of intervention groups: 2

$6 \%$ hydrogen peroxide paint-on gel

Placebo: non-whitening toothpaste

Duration of treatment: 2 weeks

Improvement in tooth colour

Vita shade guide: C1: lightest (1 rank) - B4: darkest (16 rank)

Notes

Sample size calculation: not reported

Adverse effects: sensitivity

Health-related quality of life: not reported

Key conclusions of the study authors: "The self-applied tooth-whitening gel containing $6 \%$ hydrogen peroxide has been shown to significantly improve the whiteness of teeth after 1 and 2 weeks of product use, compared to the baseline and the toothpaste only group" 


\section{Correspondence required: no}

Contact: Dr Luisa Collins; luisa.z.collins@unilever.com

\section{Risk of bias}

\begin{tabular}{|c|c|c|}
\hline Bias & Authors' judgement & Support for judgement \\
\hline $\begin{array}{l}\text { Random sequence genera- } \\
\text { tion (selection bias) }\end{array}$ & Unclear risk & $\begin{array}{l}\text { Quote: "Subjects were stratified based on their gender and age and randomly } \\
\text { assigned." However, the method is not mentioned }\end{array}$ \\
\hline $\begin{array}{l}\text { Allocation concealment } \\
\text { (selection bias) }\end{array}$ & Unclear risk & Not mentioned \\
\hline $\begin{array}{l}\text { Blinding of participants } \\
\text { and personnel (perfor- } \\
\text { mance bias) } \\
\text { All outcomes }\end{array}$ & Unclear risk & $\begin{array}{l}\text { Quote: "examiner-blinded, stratified, parallel-design clinical trial." However, } \\
\text { method is not reported }\end{array}$ \\
\hline $\begin{array}{l}\text { Blinding of outcome as- } \\
\text { sessment (detection bias) } \\
\text { All outcomes }\end{array}$ & Unclear risk & $\begin{array}{l}\text { Quote: "examiner-blinded, stratified, parallel-design clinical trial." However, } \\
\text { method is not reported }\end{array}$ \\
\hline $\begin{array}{l}\text { Incomplete outcome data } \\
\text { (attrition bias) } \\
\text { All outcomes }\end{array}$ & Low risk & $\begin{array}{l}\text { Quote: ".. from the } 11 \text { subjects that failed to complete the clinical trial" } \\
\text { Comment: missing of outcome data balanced in numbers across intervention } \\
\text { groups }\end{array}$ \\
\hline $\begin{array}{l}\text { Selective reporting (re- } \\
\text { porting bias) }\end{array}$ & Low risk & $\begin{array}{l}\text { All outcomes described were reported. Conclusions are in accordance with the } \\
\text { results }\end{array}$ \\
\hline Other bias & Low risk & None \\
\hline
\end{tabular}

Costa 2012

\begin{tabular}{ll}
\hline Methods & Title: comparison of 2 at-home whitening products of similar peroxide concentration and different de- \\
livery methods & Trial design: randomised, single-blinded, split-mouth design \\
Location: not reported & Language: English \\
& Number of centres: 1 \\
& Recruitment period: not reported \\
& Funding source: Ultradent \\
& Participants: 21 to 75 years old \\
Total number: 25 \\
Inclusion criteria: \\
- be at least 18 years old \\
- willing to sign a consent form \\
Participants
\end{tabular}


Costa 2012 (Continued)

- presence of all 6 maxillary teeth equal or darker than 1M2 VITA bleached guide in the value

- have no maxillary anterior teeth with more than $1 / 6$ of the facial surface covered with a restoration

Exclusion criteria:

- history of any medical disease that may interfere with the study or require special consideration

- presence of gross pathology

- use of tobacco products during previous 30 days

- current or previous use of whitening agent

- Loë and Silness 29 gingival score $>1.0$

- pregnant or lactating women

- tetracycline-stained teeth

Number randomised: 25

Method of randomisation: not reported

Method of allocation concealment: not reported

Method of blinding: not reported

Number evaluated: 24

$\begin{array}{ll}\text { Interventions } & \text { Total number of intervention groups: } 2 \\ 35 \% \text { carbamide peroxide } \\ 14 \% \text { hydrogen peroxide } \\ \text { Duration of treatment: } 2 \text { weeks }\end{array}$

Outcomes Improvement in tooth shade: $a^{\star} . b^{\star}$ and $L$ were recorded. Whitening benefit was represented by negative $b$ (yellowness reduction), and positive $\Delta \mathrm{L}$ (increasing lightness)

Sensitivity: VAS scale: 1 no pain, 10 severe pain

Adverse effects: sensitivity

Health-related quality of life: not reported

Key conclusions of the study authors: "There was no significant difference in tooth colour change between carbamide peroxide and hydrogen peroxide at either time point. By the end of the study no participants reported tooth and gingival sensitivity. Participants preferred CP over HP"

Contact: Dr D Costa; dacostaj@ohsu.edu

\section{Risk of bias}

\section{Bias}

Random sequence genera- Unclear risk tion (selection bias)

\section{Authors' judgement Support for judgement}

Quote: "randomised, single-blinded, split-mouth design clinical study." However, method is not reported

Not mentioned

Quote: "randomised, single-blinded, split-mouth design clinical study." However, method is not reported 
Costa 2012 (Continued)

All outcomes

\begin{tabular}{|c|c|c|}
\hline $\begin{array}{l}\text { Blinding of outcome as- } \\
\text { sessment (detection bias) } \\
\text { All outcomes }\end{array}$ & Unclear risk & $\begin{array}{l}\text { Quote: "randomised, single-blinded, split-mouth design clinical study." How- } \\
\text { ever, method is not reported }\end{array}$ \\
\hline $\begin{array}{l}\text { Incomplete outcome data } \\
\text { (attrition bias) } \\
\text { All outcomes }\end{array}$ & Low risk & $\begin{array}{l}\text { Quote: "A total of } 25 \text { participants enrolled and } 24 \text { completed the study" } \\
\text { Comment: plausible effect size (difference in means) among missing outcomes } \\
\text { not enough to have a clinically relevant impact on observed effect size }\end{array}$ \\
\hline $\begin{array}{l}\text { Selective reporting (re- } \\
\text { porting bias) }\end{array}$ & Low risk & $\begin{array}{l}\text { All outcomes described were reported. Conclusions are in accordance with the } \\
\text { results }\end{array}$ \\
\hline Other bias & Low risk & None \\
\hline
\end{tabular}

\section{Cronin 2005}

Methods Title: comparison of 2 over-the-counter tooth whitening products using a novel system

Trial design: observer-blinded, parallel-group randomised controlled trial

Location: not reported

Language: English

Number of centres: 1

Recruitment period: not reported

Funding source: sponsored by Pfizer

Participants

Participants: 18 years old and above

Total number: 60

Inclusion criteria:

- A2 or darker on 2 or 4 incisors

Exclusion criteria:

- restoration covering more than $25 \%$ of the facial surface of maxillary anterior teeth

- orthodontic bands

- significant oral pathology

- professionally whitened teeth in the last 6 months

Number randomised: 60

Method of randomisation: not reported

Method of allocation concealment: not reported

Method of blinding: not reported

Number evaluated: 59

Interventions

Total number of intervention groups: 2

$6 \%$ hydrogen peroxide 
Cronin 2005 (Continued)

$18 \%$ carbamide peroxide

Duration of treatment: 2 weeks

\begin{tabular}{|c|c|}
\hline \multirow[t]{2}{*}{ Outcomes } & Improvement in tooth shade: shade assessment by VITAPAN C4 -1 to B1 - 16 \\
\hline & $\begin{array}{l}\Delta \mathrm{L}, \mathrm{a}^{\star}, \mathrm{b}^{\star} \text { were recorded (b: decreased } \mathrm{b}^{\star} \text { indicates reduced yellowness; } \Delta \mathrm{L} \text { : increased } \Delta \mathrm{L} \text { is increased } \\
\text { brightness) }\end{array}$ \\
\hline
\end{tabular}

Sotes
Adverse effects: sensitivity
Health-related quality of life: not reported
Key conclusions of the study authors: "Both the treatment showed significant improvement in tooth
shade. $6 \%$ hydrogen peroxide showed better improvement when compared to carbamide peroxide"
Correspondence required: no
Contact: Martin J Cronin, Director Dental Research, New Institutional Service Co, Northfield, New Jer-
sey, USA

\section{Risk of bias}

\begin{tabular}{|c|c|c|}
\hline Bias & Authors' judgement & Support for judgement \\
\hline $\begin{array}{l}\text { Random sequence genera- } \\
\text { tion (selection bias) }\end{array}$ & Unclear risk & $\begin{array}{l}\text { Quote: "Qualifying subjects were stratified and randomly assigned to } 1 \text { of the } 2 \\
\text { test products." However, method is not reported }\end{array}$ \\
\hline $\begin{array}{l}\text { Allocation concealment } \\
\text { (selection bias) }\end{array}$ & Unclear risk & Not mentioned \\
\hline $\begin{array}{l}\text { Blinding of participants } \\
\text { and personnel (perfor- } \\
\text { mance bias) } \\
\text { All outcomes }\end{array}$ & Unclear risk & $\begin{array}{l}\text { Quote: "examiner-blinded, randomised, parallel-group study." However, } \\
\text { method is not reported }\end{array}$ \\
\hline $\begin{array}{l}\text { Blinding of outcome as- } \\
\text { sessment (detection bias) } \\
\text { All outcomes }\end{array}$ & Unclear risk & $\begin{array}{l}\text { Quote: "examiner-blinded, randomised, parallel-group study." However, } \\
\text { method is not reported }\end{array}$ \\
\hline $\begin{array}{l}\text { Incomplete outcome data } \\
\text { (attrition bias) } \\
\text { All outcomes }\end{array}$ & Low risk & $\begin{array}{l}\text { Quote: "Of } 60 \text { enrolled in study with } 59 \text { completing the study" } \\
\text { Comment: plausible effect size (difference in means or standardised difference } \\
\text { in means) among missing outcomes not enough to have a clinically relevant } \\
\text { impact on observed effect size }\end{array}$ \\
\hline $\begin{array}{l}\text { Selective reporting (re- } \\
\text { porting bias) }\end{array}$ & Low risk & $\begin{array}{l}\text { All outcomes described were reported. Conclusions are in accordance with the } \\
\text { results }\end{array}$ \\
\hline Other bias & Low risk & None \\
\hline
\end{tabular}

\section{Delgado 2007}

Methods

Title: tooth-whitening efficacy of custom tray-delivered $9 \%$ hydrogen peroxide and $20 \%$ carbamide peroxide during daytime use: a 14-day clinical trial

Trial design: double-blinded, randomised controlled clinical trial 
Delgado 2007 (Continued)

Location: University of Puerto Rico

Language: English

Number of centres: 1

Recruitment period: not reported

Funding source: Colgate

\section{Participants}

Participants: 25 to 64 years of age

Total number: 46

Inclusion criteria:

- healthy male and female subjects aged 21 to 68 years

- availability for the 2 weeks duration of the study

- minimum average Vita shade of A3 for all 6 maxillary anterior teeth

- 6 natural maxillary anterior teeth must be present and free of large restorations or extrinsic stains covering more than $1 / 3$ of the facial tooth surface or a maximum of 1 dental prosthetic crown/facial veneer

Exclusion criteria:

- presence of orthodontic appliances

- presence of tumours or significant pathology of the soft or hard tissues of the oral cavity

- presence of moderate or advanced periodontal disease (ADA III or IV)

- presence of 5 or more carious lesions requiring immediate care

- use of stain-inducing medications or oral use products 1 month prior to, or anytime during, the 2 weeks of the study

- participation in any other clinical study or test panel in the last month

- pregnant or lactating women

- allergies to tooth whitening products, personal care consumer products, or their ingredients

Number randomised: 46

Method of randomisation: random list

Method of allocation concealment: not reported

Method of blinding: not reported

Number evaluated: 37

Total number of intervention groups: 2

Group I: Colgate Visible White containing 9\% hydrogen peroxide (9\% HP)

Group II: Opalescence containing 20\% carbamide peroxide (20\% CP)

Duration of treatment: 30 minutes for 2 weeks

Outcomes Improvement in tooth shade

The shade guide tabs were arranged B1 to C4 representing the 1 to 16 scale

Notes Sample size calculation: not reported

Adverse effects: gingival irritation

Health-related quality of life: not reported 
Key conclusions of the study authors: "Both 9\% hydrogen peroxide and 20\% carbamide peroxide products effectively whitened teeth after 5,7 and 14 days of once-a day 30 -minute applications. $9 \%$ hydrogen peroxide produced a statistically significant tooth shade improvement compared to the tooth whitening effect of $20 \%$ carbamide peroxide after 5 days of product use. Colgate Visible White $9 \%$ hydrogen peroxide and Opalescence ( $20 \%$ carbamide peroxide) had a similar whitening effect after 7 and 14 days of use. Both tooth whitening products tested produced little tooth sensitivity or gingival irritation"

Contact: Evaristo Delgado; edelgadoc@yahoo.com

\section{Risk of bias}

\begin{tabular}{|c|c|c|}
\hline Bias & Authors' judgement & Support for judgement \\
\hline $\begin{array}{l}\text { Random sequence genera- } \\
\text { tion (selection bias) }\end{array}$ & Low risk & $\begin{array}{l}\text { Quote: "Participants were assigned following a random list to } 1 \text { of the } 2 \text { treat- } \\
\text { ment groups" }\end{array}$ \\
\hline $\begin{array}{l}\text { Allocation concealment } \\
\text { (selection bias) }\end{array}$ & Unclear risk & Not mentioned \\
\hline $\begin{array}{l}\text { Blinding of participants } \\
\text { and personnel (perfor- } \\
\text { mance bias) } \\
\text { All outcomes }\end{array}$ & Unclear risk & $\begin{array}{l}\text { Quote: "randomised, single-centre, parallel-group, double-blinded clinical tri- } \\
\text { al." However, method is not reported }\end{array}$ \\
\hline $\begin{array}{l}\text { Blinding of outcome as- } \\
\text { sessment (detection bias) } \\
\text { All outcomes }\end{array}$ & Unclear risk & $\begin{array}{l}\text { Quote: "randomised, single-centre, parallel-group, double-blinded clinical tri- } \\
\text { al." However, method is not reported }\end{array}$ \\
\hline $\begin{array}{l}\text { Incomplete outcome data } \\
\text { (attrition bias) } \\
\text { All outcomes }\end{array}$ & Unclear risk & $\begin{array}{l}\text { Quote: "9 dropouts noted" } \\
\text { Comment: } 7 \text { failed to keep up with study visits. Unbalanced number in both } \\
\text { groups ( } 20 \% \text { CP: } n=16 \text { and } 9 \% \text { HP: } n=21 \text { ). We are not sure if plausible effect } \\
\text { size (difference in means) among missing outcomes may have a clinically rele- } \\
\text { vant impact on observed effect size }\end{array}$ \\
\hline $\begin{array}{l}\text { Selective reporting (re- } \\
\text { porting bias) }\end{array}$ & Low risk & $\begin{array}{l}\text { All outcomes described were reported. Conclusions are in accordance with the } \\
\text { results }\end{array}$ \\
\hline Other bias & Low risk & None \\
\hline
\end{tabular}

\section{Ferrari 2007}

Methods

Title: daytime use of a custom bleaching tray or whitening strips: initial and sustained colour improvement

Trial design: randomised, parallel, examiner-blinded study

Location: Livorno, Italy

Language: English

Number of centres: 1

Recruitment period: not reported

Funding source: Procter \& Gamble

Participants Participants: 19 to 56 years old. Mean age 32.8 years


Ferrari 2007 (Continued)

Total number: 43

Inclusion criteria:

- 18 years of age and older

- no history of tooth whitening

- no current tooth sensitivity

Exclusion criteria: not reported

Number randomised: not reported

Method of randomisation: not reported

Method of allocation concealment: not reported

Method of blinding: blinded kit boxes

Number evaluated: 36

Interventions

Total number of intervention groups: 2

$6 \%$ hydrogen peroxide strips

$10 \%$ carbamide peroxide tray

Duration of treatment: 2 weeks

\begin{tabular}{|c|c|}
\hline Outcomes & $\begin{array}{l}\text { Colour improvement } \\
a^{\star} . b^{\star} \text { and } \Delta L \text { were recorded } \\
\text { Whitening benefit was represented by negative } b^{\star} \text { (yellowness reduction), and positive } \Delta L \text { (increasing } \\
\text { lightness) }\end{array}$ \\
\hline Notes & $\begin{array}{l}\text { Adverse effects: tooth sensitivity and gingival irritation } \\
\text { Health-related quality of life: not reported } \\
\text { Key conclusions of the study authors: "The strip system yielded significant reduction in yellowness } \\
\text { compared to the custom tray, at both end-of-treatment and post-treatment monitoring. Compared to } \\
\text { Week } 2 \text {, the strip group retained } 89 \%-92 \% \text { of the initial colour improvement at Week } 6 \text { ( } 4 \text { weeks post- } \\
\text { treatment), while the tray group had } 80 \%-90 \% \text {. Both daytime treatments were well-tolerated, with mi- } \\
\text { nor tooth sensitivity and oral irritation representing the most common findings" } \\
\text { Correspondence required: no } \\
\text { Contact: Professor Dr Marco Ferrari; ferrarimar@unisi.it }\end{array}$ \\
\hline
\end{tabular}

\section{Risk of bias}

\begin{tabular}{lll}
\hline Bias & Authors' judgement & Support for judgement \\
\hline $\begin{array}{l}\text { Random sequence genera- } \\
\text { tion (selection bias) }\end{array}$ & Unclear risk & $\begin{array}{l}\text { Quote: "randomised, parallel, examiner-blinded study." However, method of } \\
\text { randomisation is not reported }\end{array}$ \\
\hline $\begin{array}{l}\text { Allocation concealment } \\
\text { (selection bias) }\end{array}$ & Unclear risk & Not mentioned \\
\hline $\begin{array}{l}\text { Blinding of participants } \\
\text { and personnel (perfor- } \\
\text { mance bias) }\end{array}$ & Low risk & $\begin{array}{l}\text { Quote: "Because of the dissimilar delivery systems, test products were sup- } \\
\text { plied in blinded kit boxes" }\end{array}$ \\
\hline
\end{tabular}


Ferrari 2007 (Continued)

All outcomes

\begin{tabular}{|c|c|c|}
\hline $\begin{array}{l}\text { Blinding of outcome as- } \\
\text { sessment (detection bias) }\end{array}$ & Unclear risk & $\begin{array}{l}\text { Quote: "randomised, parallel, examiner-blinded study." However, method of } \\
\text { blinding is not reported }\end{array}$ \\
\hline
\end{tabular}

All outcomes

$\begin{array}{ll}\begin{array}{l}\text { Incomplete outcome data } \\ \text { (attrition bias) }\end{array} & \text { Low risk } \\ \text { All outcomes } & \text { week visit, while } 1 \text { additional subject (in the tray group) missed the.." } \\ & \begin{array}{l}\text { Comment: } 7 \text { dropouts were reported. HP strips group } \mathrm{n}=17 \text { and CP tray group } \\ \mathrm{n}=19 . \text { Missing outcome data balanced in numbers across intervention groups }\end{array}\end{array}$

\begin{tabular}{lll}
\hline $\begin{array}{l}\text { Selective reporting (re- } \\
\text { porting bias) }\end{array}$ & Low risk & $\begin{array}{l}\text { All outcomes described were reported. Conclusions are in accordance with the } \\
\text { results }\end{array}$ \\
\hline Other bias & Low risk & None \\
\hline
\end{tabular}

\section{Gallo 2009}

Title: evaluation of $30 \%$ carbamide peroxide at-home bleaching gels with and without potassium ni-
trate
Trial design: double-blinded, randomised controlled trial
Location: not reported
Language: English
Number of centres: 1
Recruitment period: not reported
Funding source: Dent Mat

Participants: adults
Total number: 40
Inclusion criteria:
- shade darker than B65
- no sensitive teeth
- not used a bleaching material in 5 years
Exclusion criteria:
- unable to meet the time requirements
- reported cold-sensitive teeth
- pregnant or nursing
Number randomised: 40
Method of randomisation: not mentioned
Method of allocation concealment: not mentioned
Method of blinding: not reported
Number evaluated: 40


Gallo 2009 (Continued)

Interventions

Total number of intervention groups: 2

$30 \%$ carbamide peroxide with $5 \%$ potassium nitrate in tray

$30 \%$ carbamide peroxide in tray

Duration of treatment: 1 hour per day for 10 days

\section{Outcomes}

Change in tooth shade: Bioform Colour Ordered Shade Guide System: B85 darkest (1) to B59 lightest (24)

Sensitivity of tooth: 0 to 10 scale (0: no pain, 10 : severe)

Gingival irritation: 0 normal, 1 mild, 2 moderate, 3 severe

Sotes
Adverse effects: sensitivity and gingiva irritation
Health-related quality of life: not reported
Key conclusions of the study authors: "30\% carbamide bleaching gels effectively whiten teeth without
causing a significant increase in tooth sensitivity or changes in gingival condition. Potassium nitrate
has little effect in sensitivity when treatment time is short"
Correspondence required: no
Contact: Dr John Gallo; jgallo@lsuhsc.edu

\section{Risk of bias}

\begin{tabular}{|c|c|c|}
\hline Bias & Authors' judgement & Support for judgement \\
\hline $\begin{array}{l}\text { Random sequence genera- } \\
\text { tion (selection bias) }\end{array}$ & Unclear risk & $\begin{array}{l}\text { Quote: "this double-blinded, randomised clinical study evaluated the effec- } \\
\text { tiveness..." However, method of randomisation not reported }\end{array}$ \\
\hline $\begin{array}{l}\text { Allocation concealment } \\
\text { (selection bias) }\end{array}$ & Unclear risk & Not mentioned \\
\hline $\begin{array}{l}\text { Blinding of participants } \\
\text { and personnel (perfor- } \\
\text { mance bias) } \\
\text { All outcomes }\end{array}$ & Unclear risk & $\begin{array}{l}\text { Quote: "Neither subjects nor evaluators knew which bleaching gel (treatment } \\
\text { A..... was used.." However, method of blinding not reported }\end{array}$ \\
\hline $\begin{array}{l}\text { Blinding of outcome as- } \\
\text { sessment (detection bias) } \\
\text { All outcomes }\end{array}$ & Unclear risk & $\begin{array}{l}\text { Quote: "Neither subjects nor evaluators knew which bleaching gel (treatment } \\
\text { A..... was used.." However, method of blinding not reported }\end{array}$ \\
\hline $\begin{array}{l}\text { Incomplete outcome data } \\
\text { (attrition bias) } \\
\text { All outcomes }\end{array}$ & Low risk & No mention of dropouts \\
\hline $\begin{array}{l}\text { Selective reporting (re- } \\
\text { porting bias) }\end{array}$ & Low risk & $\begin{array}{l}\text { All outcomes described were reported. Conclusions are in accordance with the } \\
\text { results }\end{array}$ \\
\hline Other bias & Low risk & None \\
\hline
\end{tabular}


Methods

Title: placebo-controlled, 6-week clinical trial on the safety and efficacy of a low-gel, $14 \%$ hydrogen-peroxide whitening strip

Trial design: parallel-group, double-blinded, randomised controlled trial

Location: not reported

Language: English

Number of centres: 1

Recruitment period: not reported

Funding source: Procter \& Gamble

Participants

Participants: consenting adults, mean age: 32.1 years

Total number: 39

Inclusion criteria:

- A2 or darker shade

- healthy adults with no sensitivity

Exclusion criteria:

- previous bleaching

- restoration

Number randomised: 39

Method of randomisation: not reported

Method of allocation concealment: not reported

Method of blinding: not reported

Number evaluated: 35.4 dropouts, 2 from each group

Total number of intervention groups: 2

Experiment: $14 \%$ hydrogen peroxide

Control: placebo

Duration of treatment: 3 weeks

Improvement in tooth colour
$\mathrm{b}^{\star}:$ decreased $\mathrm{b}^{*}$ indicates reduced yellowness
$\Delta \mathrm{L}$ : increased $\Delta \mathrm{L}$ is increased brightness
$\Delta \mathrm{W}$ : negative $\mathrm{W}$ indicates colour closer to white

Notes

Sample size calculation: not reported

Adverse effects: tooth sensitivity and oral irritation

Health-related quality of life: not reported

Key conclusions of the study authors: "Twice-daily use of Crest Whitestrips Supreme resulted in a highly significant improvement in tooth colour after 3 weeks, with colour improvement continuing over 6 weeks" 
Garcia-Godoy 2004 (Continued)

Risk of bias

\begin{tabular}{|c|c|c|}
\hline Bias & Authors' judgement & Support for judgement \\
\hline $\begin{array}{l}\text { Random sequence genera- } \\
\text { tion (selection bias) }\end{array}$ & Unclear risk & $\begin{array}{l}\text { Quote: "randomised double-blinded parallel-group clinical trial. Eligible sub- } \\
\text { jects were randomised to a low gel } 14 \% \text {...or placebo group." However, method } \\
\text { of randomisation is not mentioned }\end{array}$ \\
\hline $\begin{array}{l}\text { Allocation concealment } \\
\text { (selection bias) }\end{array}$ & Unclear risk & Not mentioned \\
\hline $\begin{array}{l}\text { Blinding of participants } \\
\text { and personnel (perfor- } \\
\text { mance bias) } \\
\text { All outcomes }\end{array}$ & Unclear risk & $\begin{array}{l}\text { Quote: "randomised double-blinded parallel-group clinical trial." However, } \\
\text { method of blinding is not mentioned }\end{array}$ \\
\hline $\begin{array}{l}\text { Blinding of outcome as- } \\
\text { sessment (detection bias) } \\
\text { All outcomes }\end{array}$ & Unclear risk & $\begin{array}{l}\text { Quote: "randomised double-blinded parallel-group clinical trial." However, } \\
\text { method of blinding is not mentioned }\end{array}$ \\
\hline $\begin{array}{l}\text { Incomplete outcome data } \\
\text { (attrition bias) } \\
\text { All outcomes }\end{array}$ & Low risk & $\begin{array}{l}\text { Quote: "2 subjects } 1 \text { in each group discontinued treatment because of a treat- } \\
\text { ment related adverse event" } \\
\text { Comment: missing outcome data balanced in numbers across intervention } \\
\text { groups }\end{array}$ \\
\hline $\begin{array}{l}\text { Selective reporting (re- } \\
\text { porting bias) }\end{array}$ & Low risk & $\begin{array}{l}\text { All outcomes described were reported. Conclusions are in accordance with the } \\
\text { results }\end{array}$ \\
\hline Other bias & Low risk & None \\
\hline
\end{tabular}

Gerlach 2000

Methods Title: a randomised clinical trial comparing a novel $5.3 \%$ hydrogen peroxide whitening strip to $10 \%$, $15 \%$, and $20 \%$ carbamide peroxide tray-based bleaching system

Trial design: randomised controlled, examiner-blinded, parallel-group clinical trial

Location: not reported

Language: English

Number of centres: 1

Recruitment period: not reported

Funding source: Procter \& Gamble

Participants

Participants: 24 to 57 years old. Mean age: 42.8 years

Total number: 36

Inclusion criteria:

- adults willing to whiten teeth

Exclusion criteria:

- previous report of sensitivity 
Gerlach 2000 (Continued)

- large restoration

- history of tooth whitening

Number randomised: 36

Method of randomisation: not reported

Method of allocation concealment: not reported

Method of blinding: not reported

Number evaluated: 32

\begin{tabular}{|c|c|}
\hline \multirow[t]{6}{*}{ Interventions } & Total number of intervention groups: 4 \\
\hline & $5.3 \%$ hydrogen peroxide strip \\
\hline & $10 \%$ carbamide peroxide gel in tray \\
\hline & $15 \%$ carbamide peroxide gel in tray \\
\hline & $20 \%$ carbamide peroxide gel in tray \\
\hline & Duration of treatment: 14 days, 2 hours per day \\
\hline \multirow[t]{2}{*}{ Outcomes } & Improvement in tooth colour \\
\hline & $\Delta \mathrm{L}, \mathrm{a}^{*}, \mathrm{~b}^{*}$ values were recorded. Increase in $\Delta \mathrm{L}$ and reduction $\mathrm{b}^{*}$ indicated whitening \\
\hline \multirow[t]{6}{*}{ Notes } & Sample size calculation: not reported \\
\hline & Adverse effects: sensitivity \\
\hline & Health-related quality of life: not reported \\
\hline & $\begin{array}{l}\text { Key conclusions of the study authors: ".. all groups experienced a greater than } 1 \text {-unit mean improve- } \\
\text { ment in all parameters relative to baseline. For the primary study variable, reduction of yellow (delta } \\
b^{\star} \text { ) outcomes after } 14 \text { hours of using the experimental strip were comparable to those observed with } \\
\text { the } 10 \% \text { tray group after } 28 \text { hours of use. These } 2 \text { treatment groups did not differ statistically with re- } \\
\text { spect to any of the colour measurements used in this study. For the tray groups, there was a reasonable } \\
\text { dose relationship for the primary endpoint, delta b*, with the } 15 \% \text { and } 20 \% \text { tray groups averaging } 17 \% \\
\text { and } 68 \% \text { improvements in yellow, respectively, over the } 10 \% \text { group. Except for the } 20 \% \text { carbamide per- } \\
\text { oxide system, where sensitivity was relatively common, all test products were well tolerated" }\end{array}$ \\
\hline & Correspondence required: no \\
\hline & $\begin{array}{l}\text { Contact: Dr Robert W Gerlach, } 8700 \text { Mason Montgomery Road, Mason OH, } 450408006 \text { USA; gerlach.r- } \\
\text { w@pg.com }\end{array}$ \\
\hline
\end{tabular}

\section{Risk of bias}

\begin{tabular}{lll}
\hline Bias & Authors' judgement & Support for judgement \\
\hline $\begin{array}{l}\text { Random sequence genera- } \\
\text { tion (selection bias) }\end{array}$ & Unclear risk & $\begin{array}{l}\text { Quote: "randomised controlled, examiner-blinded, parallel group." However, } \\
\text { method is not reported }\end{array}$ \\
\hline $\begin{array}{l}\text { Allocation concealment } \\
\text { (selection bias) }\end{array}$ & Unclear risk & Not mentioned \\
\hline $\begin{array}{l}\text { Blinding of participants } \\
\text { and personnel (perfor- } \\
\text { mance bias) }\end{array}$ & Unclear risk & Not Mentioned \\
\hline
\end{tabular}


Gerlach 2000 (Continued)

All outcomes

\begin{tabular}{|c|c|c|}
\hline $\begin{array}{l}\text { Blinding of outcome as- } \\
\text { sessment (detection bias) }\end{array}$ & Unclear risk & $\begin{array}{l}\text { Quote: "randomised controlled, examiner-blinded, parallel group." However } \\
\text { method is not reported }\end{array}$ \\
\hline
\end{tabular}

All outcomes

\begin{tabular}{lll}
$\begin{array}{l}\text { Incomplete outcome data } \\
\text { (attrition bias) } \\
\text { All outcomes }\end{array}$ & Low risk & $\begin{array}{l}\text { Quote: "4 participants missed day 14" } \\
\text { Comment: missing outcome data balanced in numbers across intervention } \\
\text { groups }(\mathrm{n}=9 \text { in HP strip and } \mathrm{n}=8 \text { in CP tray group) }\end{array}$ \\
\hline $\begin{array}{l}\text { Selective reporting (re- } \\
\text { porting bias) }\end{array}$ & Low risk & $\begin{array}{l}\text { All outcomes described were reported. Conclusions are in accordance with the } \\
\text { results }\end{array}$ \\
\hline Other bias & Low risk & None
\end{tabular}

\section{Gerlach 2002}

Methods Title: initial colour change and colour retention with a hydrogen peroxide bleaching strip

Trial design: randomised double-blinded design

Location: not reported

Language: English

Number of centres: 1

Recruitment period: not reported

Funding source: Procter \& Gamble. The authors are employees

\section{Participants}

Participants: 18 to 71 years old. Mean age: 40.9 years

Total number: 57

Inclusion criteria:

- 18 years and above

- no history of bleaching, sensitivity or restorative dentistry

Exclusion criteria:

- dental prophylaxis in last 3 months

Number randomised: 57

Method of randomisation: not reported

Method of allocation concealment: not reported

Method of blinding: identically labelled and packaged, differing in appearance only as to a unique subject number

Number evaluated: 52 completed study and 49 completed follow-up

Interventions

Total number of intervention groups: 2

Experiment: $5.3 \%$ hydrogen peroxide - Crest white strips

Control: placebo strips 
Gerlach 2002 (Continued)

Duration of treatment: 2 weeks

\begin{tabular}{|c|c|}
\hline \multirow[t]{4}{*}{ Outcomes } & Improvement in tooth colour \\
\hline & $b^{\star}$ : decreased $b^{\star}$ indicates reduced yellowness \\
\hline & $\Delta \mathrm{L}$ : increased $\Delta \mathrm{L}$ is increased brightness \\
\hline & $\Delta \mathrm{E}:$ overall whiteness \\
\hline
\end{tabular}

Notes

Sample size calculation: not reported

Adverse effects: tooth sensitivity and gingival irritation

Health-related quality of life: not reported

Key conclusions of the study authors: "Whitening strip group continuing to demonstrate improvements in tooth colour relative to baseline and placebo. Age was found to significantly contribute to initial colour improvement, with younger subjects experiencing a greater initial reduction in yellowness compared to older participants, but not to post-treatment colour retention. The whitening strips were well tolerated, with minor tooth sensitivity and oral irritation representing the most common findings during treatment. There were no persistent or new treatment-related adverse events during the 6-month monitoring"

Contact: Dr Robert W Gerlach, 8700 Mason Montgomery Road, Mason OH, 450408006 USA; gerlach.rw@pg.com

\section{Risk of bias}

\begin{tabular}{lll}
\hline Bias & Authors' judgement & Support for judgement \\
\hline $\begin{array}{l}\text { Random sequence genera- } \\
\text { tion (selection bias) }\end{array}$ & Unclear risk & $\begin{array}{l}\text { Quote: "randomised double-blinded placebo-controlled study.. subjects were } \\
\text { randomised to either the whitening strip or placebo strip groups." However, } \\
\text { method of randomisation is not mentioned }\end{array}$ \\
\hline $\begin{array}{l}\text { Allocation concealment } \\
\text { (selection bias) }\end{array}$ & Unclear risk & Not reported \\
\hline $\begin{array}{l}\text { Blinding of participants } \\
\text { and personnel (perfor- } \\
\text { mance bias) } \\
\text { All outcomes }\end{array}$ & Low risk & $\begin{array}{l}\text { Quote: "All products in this double-blinded clinical trial were identically la- } \\
\text { belled and packaged, differing in appearance only as to a unique subject num- } \\
\text { ber" }\end{array}$ \\
\hline
\end{tabular}

\begin{tabular}{lll}
\hline Blinding of outcome as- & Unclear risk & Not reported \\
sessment (detection bias) & \\
All outcomes &
\end{tabular}

$\begin{array}{ll}\begin{array}{l}\text { Incomplete outcome data } \\ \text { (attrition bias) }\end{array} & \text { Low risk } \\ \text { All outcomes } & 49 \text { completed the } 6 \text {-month study" } \\ & \begin{array}{l}\text { Comment: placebo group has } 29 \text { participants while hydrogen peroxide group } \\ \text { has } 23 \text { participants. In spite of this difference, the results favour the interven- } \\ \text { tion group. Hence we presume that plausible effect size (difference in means) } \\ \text { among missing outcomes may not have a clinically relevant impact on ob- } \\ \text { served effect size }\end{array}\end{array}$

\begin{tabular}{lll}
\hline $\begin{array}{l}\text { Selective reporting (re- } \\
\text { porting bias) }\end{array}$ & Low risk & $\begin{array}{l}\text { All outcomes described are reported. Conclusions are in accordance with the } \\
\text { results }\end{array}$ \\
\hline Other bias & Low risk & None \\
\hline
\end{tabular}


Trial design: randomised, parallel, examiner-blinded clinical trial

Location: not reported

Language: English

Number of centres: 1

Recruitment period: not reported

Funding source: Procter \& Gamble

Participants: 22 to 59 years old, mean age 38.25 years
Total number: 20
Inclusion criteria: no history of previous bleaching
Exclusion criteria: not reported
Number randomised: 20
Method of randomisation: not reported
Method of allocation concealment: not reported
Method of blinding: uniquely numbered subject identification label
Number evaluated: 20
Total number of intervention groups: 2 (tray versus strip)
Hydrogen peroxide $10 \%: 30$ minutes twice daily
Hydrogen peroxide $6.5 \%$ carbamide peroxide $10 \%: 2$ hours once daily
Duration of treatment: 14 days

Outcomes Improvement in tooth shade.

Colour change $\left(\Delta \mathrm{L}^{*}, \Delta \mathrm{a}^{*}\right.$, and $\left.\Delta \mathrm{b}^{\star}\right)$ was determined by comparing each post-treatment visit to baseline. Negative $\Delta \mathrm{b}^{*}$ (reduction in yellowness) and positive $\Delta \mathrm{L}^{\star}$ (increased brightness) were considered to be indicative of a whitening benefit

Adverse effects: 1 patient had mild irritation at the tip of tongue in strip group

Health-related quality of life: not reported

Key conclusions of the study authors: "Under the conditions tested, this clinical trial demonstrates that the 14-contact-hour treatment with the strip system resulted in superior whitening efficacy compared with the 28-contact-hour treatment with the tray system"

Contact: Dr Robert W Gerlach; gerlach.rw@pg.com

\section{Risk of bias}


Gerlach 2002a (Continued)

Random sequence genera- Unclear risk Quote: "randomised, examiner-blinded, clinical trial." However, method is not tion (selection bias)

\section{mentioned}

Not reported

$\begin{array}{lll}\text { Allocation concealment } & \text { Unclear risk } & \text { Not reported } \\ \text { (selection bias) }\end{array}$

(selection bias)

\begin{tabular}{|c|c|c|}
\hline $\begin{array}{l}\text { Blinding of participants } \\
\text { and personnel (perfor- } \\
\text { mance bias) }\end{array}$ & Low risk & $\begin{array}{l}\text { Quote: "Test products were over labelled with a uniquely numbered subject } \\
\text { identification label" }\end{array}$ \\
\hline
\end{tabular}

mance bias)

All outcomes

\begin{tabular}{lll}
\hline $\begin{array}{l}\text { Blinding of outcome as- } \\
\text { sessment (detection bias) } \\
\text { All outcomes }\end{array}$ & Unclear risk & $\begin{array}{l}\text { Quote: "randomised, examiner-blinded, clinical trial." However, method is not } \\
\text { mentioned }\end{array}$ \\
\hline $\begin{array}{l}\text { Incomplete outcome data } \\
\begin{array}{l}\text { (attrition bias) } \\
\text { All outcomes }\end{array}\end{array}$ & Low risk & $\begin{array}{l}\text { Quote: "All 20 subjects completed the 14- day treatment and were considered } \\
\text { eligible for evaluation for all analyses" }\end{array}$ \\
\hline $\begin{array}{l}\text { Selective reporting (re- } \\
\text { porting bias) }\end{array}$ & Low risk & $\begin{array}{l}\text { All outcomes described were reported. Conclusions are in accordance with the } \\
\text { results }\end{array}$ \\
\hline Other bias & Low risk & None \\
\hline
\end{tabular}

\section{Gerlach 2002b}

Methods

Title: comparative response of whitening strips to a low peroxide and potassium nitrate bleaching gel

Trial design: randomised, examiner-blinded clinical trial. 2 arms

Location: not reported

Language: English

Number of centres: 1

Recruitment period: not reported

Funding source: Procter \& Gamble

Participants

Participants: mean age of 37 years

Total number: 34

Inclusion criteria: be willing to have their teeth whitened

Exclusion criteria:

- patients who has undergone bleaching or restoration of the maxillary anterior dentition

- patients with ongoing tooth sensitivity

Number randomised: 34

Method of randomisation: not reported

Method of allocation concealment: not reported

Method of blinding: not repeated 


Total number of intervention groups: 2
$5 \%$ carbamide peroxide bleaching gel + potassium nitrate in custom tray: once daily application
$6 \%$ hydrogen peroxide bleaching strip: twice daily application
Duration of treatment: 7 days

\begin{tabular}{ll}
\hline Outcomes & Improvement in tooth shade \\
$\Delta \mathrm{L}, \mathrm{a}^{*}, \mathrm{~b}^{*}$ were recorded. Increase in $\Delta \mathrm{L}$ and decrease in $\mathrm{b}^{\star}$ indicates whitening \\
\hline Sotes & Sample size calculation: not reported \\
& Adverse effects: tooth sensitivity and oral irritation \\
& Health-related quality of life: not reported \\
& Key conclusions of the study authors: "Twice daily application of $6 \%$ hydrogen peroxide strip resulted \\
in better whitening compared to 1 daily application of $5 \%$ carbamide peroxide. Sensitivity was less with & $6 \%$ hydrogen peroxide" \\
Contact: gerlach.rw@pg.com
\end{tabular}

\section{Risk of bias}

\begin{tabular}{|c|c|c|}
\hline Bias & Authors' judgement & Support for judgement \\
\hline $\begin{array}{l}\text { Random sequence genera- } \\
\text { tion (selection bias) }\end{array}$ & Unclear risk & $\begin{array}{l}\text { Quote: "Study subjects were randomly assigned to } 1 \text { of } 2 \text { groups." However, } \\
\text { method is not reported }\end{array}$ \\
\hline $\begin{array}{l}\text { Allocation concealment } \\
\text { (selection bias) }\end{array}$ & Unclear risk & Not mentioned \\
\hline $\begin{array}{l}\text { Blinding of participants } \\
\text { and personnel (perfor- } \\
\text { mance bias) } \\
\text { All outcomes }\end{array}$ & Unclear risk & $\begin{array}{l}\text { Quote: "randomised examiner-blinded clinical study." However, method is not } \\
\text { reported }\end{array}$ \\
\hline $\begin{array}{l}\text { Blinding of outcome as- } \\
\text { sessment (detection bias) } \\
\text { All outcomes }\end{array}$ & Unclear risk & $\begin{array}{l}\text { Quote: "randomised examiner-blinded clinical study." However, method is not } \\
\text { reported }\end{array}$ \\
\hline $\begin{array}{l}\text { Incomplete outcome data } \\
\text { (attrition bias) } \\
\text { All outcomes }\end{array}$ & Low risk & $\begin{array}{l}\text { Quote: "2 subjects missed the day } 7 \text { visit" } \\
\text { Comment: missing outcome data balanced in numbers across intervention } \\
\text { groups }\end{array}$ \\
\hline $\begin{array}{l}\text { Selective reporting (re- } \\
\text { porting bias) }\end{array}$ & Low risk & $\begin{array}{l}\text { All outcomes described were reported. Conclusions are in accordance with the } \\
\text { results }\end{array}$ \\
\hline Other bias & Low risk & None \\
\hline
\end{tabular}

\section{Gerlach 2003}


Trial design: blinded randomised controlled trial

Location: not reported

Language: English

Number of centres: 1

Recruitment period: not reported

Funding source: Procter \& Gamble

Participants: mean age 40.3 years
Total number: 57
Inclusion criteria:
- A2 or darker
Exclusion criteria:
- prior bleaching
- sensitivity in tooth
Number randomised: 57
Method of randomisation: not reported
Method of allocation concealment: not reported
Method of blinding: test products were over packaged in kit boxes that were labelled only with a unique
subject identification number to assure blinding
Number evaluated: 56,1 dropout

Interventions

Total number of intervention groups: 2

Crest Night Effects 19\% sodium percarbonate

Colgate Simply White Night $8.7 \%$ hydrogen peroxide

Duration of treatment: 14 nights

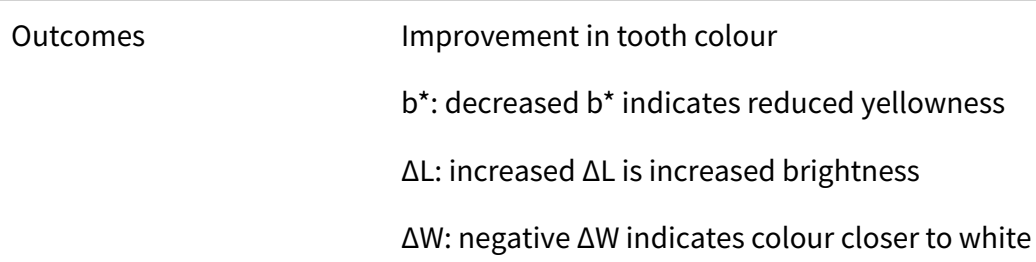

Notes

Sample size calculation: not reported

Adverse effects: sensitivity

Health-related quality of life: not reported

Key conclusions of the study authors: "After 14 nights treatment, adjusted mean (SE) change in yellowness (delta $b^{\star}$ ) was $-0.95(0.092)$ for the $19 \%$ sodium percarbonate film and $-0.17(0.096)$ for the $8.7 \%$ hydrogen peroxide gel, with these groups differing statistically $(P<0.0001)$. Other individual and composite colour parameters also demonstrated significantly greater whitening for the $19 \%$ sodium percarbonate film compared to the $8.7 \%$ hydrogen peroxide gel after 14 nights use. Only the $19 \%$ sodium percarbonate film exhibited significant $(P<0.0001)$ proximal colour improvement (delta $\left.b^{\star}\right)$ after 2 weeks, 
approximately $98 \%$ of that seen on the body of the tooth, providing evidence of proximal bleaching and uniform spatial whitening following use of this barrier-free system. Both products were well-tolerated, with no subjects discontinuing treatment early due to a causal adverse event

PMID: 15055983

Contact: Dr Robert W Gerlach, Procter and Gamble Company, 8700 Mason Montgomery Road, Mason OH 450408006 USA; gerlach.rw@pg.com

\section{Risk of bias}

\begin{tabular}{|c|c|c|}
\hline Bias & Authors' judgement & Support for judgement \\
\hline $\begin{array}{l}\text { Random sequence genera- } \\
\text { tion (selection bias) }\end{array}$ & Unclear risk & $\begin{array}{l}\text { Quote: "Blinded randomised controlled trial." However, method of randomisa- } \\
\text { tion is not mentioned }\end{array}$ \\
\hline $\begin{array}{l}\text { Allocation concealment } \\
\text { (selection bias) }\end{array}$ & Unclear risk & Method not mentioned \\
\hline $\begin{array}{l}\text { Blinding of participants } \\
\text { and personnel (perfor- } \\
\text { mance bias) } \\
\text { All outcomes }\end{array}$ & Low risk & $\begin{array}{l}\text { Quote: "Test products were over packaged in kit boxes that were labelled only } \\
\text { with a unique subject identification number to assure blinding" }\end{array}$ \\
\hline $\begin{array}{l}\text { Blinding of outcome as- } \\
\text { sessment (detection bias) } \\
\text { All outcomes }\end{array}$ & Unclear risk & $\begin{array}{l}\text { Quote: "double-blinded, randomised, controlled, parallel-group clinical trial." } \\
\text { However, method of blinding not reported }\end{array}$ \\
\hline $\begin{array}{l}\text { Incomplete outcome data } \\
\text { (attrition bias) } \\
\text { All outcomes }\end{array}$ & Low risk & $\begin{array}{l}\text { Quote: "1 subject discontinued the study" } \\
\text { Comment: plausible effect size (difference in means) among missing outcomes } \\
\text { not enough to have a clinically relevant impact on observed effect size }\end{array}$ \\
\hline $\begin{array}{l}\text { Selective reporting (re- } \\
\text { porting bias) }\end{array}$ & Low risk & $\begin{array}{l}\text { All outcomes described were reported. Conclusions are in accordance with the } \\
\text { results }\end{array}$ \\
\hline Other bias & Low risk & None \\
\hline
\end{tabular}

Gerlach 2004

$\begin{array}{ll}\text { Methods } & \text { Title: clinical trial comparing } 2 \text { daytime hydrogen-peroxide professional vital-bleaching systems } \\ \text { Trial design: randomised, examiner-blinded clinical trial } \\ \text { Location: USA } \\ \text { Language: English } \\ \text { Number of centres: } 1 \\ \text { Recruitment period: not reported } \\ \text { Funding source: Procter \& Gamble } \\ \text { Participants: adults aged } 18 \text { to } 64 \\ \text { Total number: } 31 \\ \text { Inclusion criteria: }\end{array}$


- healthy adults aged 18 or older who have 3 or more maxillary anterior teeth with a tooth shade of A2 or darker, as measured by using a standard 16-tab system

Exclusion criteria:

- prosthetic or orthodontic appliances on the maxillary dentition, dentine hypersensitivity, or a history of vital bleaching

Number randomised: 31

Method of randomisation: not reported

Method of allocation concealment: unclear

Method of blinding: unclear

Number evaluated: 29

\begin{tabular}{ll}
\hline Interventions & Total number of intervention groups: 2. \\
$14 \%$ hydrogen peroxide whitening strips \\
$9.5 \%$ hydrogen peroxide custom-tray-based system \\
Duration of treatment: 22 days
\end{tabular}

Outcomes Improvement in tooth colour: $\Delta \mathrm{L}, \mathrm{a}^{\star}, \mathrm{b}^{\star}$ values were recorded. Increase in $\mathrm{L}$ and reduction $\mathrm{b}^{\star}$ indicated whitening

Notes Sample size calculation: not reported

Adverse effects: tooth sensitivity and oral irritations were the most common side effects. $28 \%$ of the patients reported 1 or both. Occurrence of either sensitivity or irritation was $13 \%$ in strip group, $56 \%$ in tray group

Health-related quality of life: not reported

Key conclusions of the study authors: "At the end of the treatment, the $14 \%$ hydrogen peroxide whitening strips caused 2-fold reduction in yellowness and better in-use tolerability when compared to $9.5 \%$ hydrogen peroxide custom-tray system"

Correspondence required: no

Contact: gerlach.rw@pg.com

\section{Risk of bias}

\begin{tabular}{lll}
\hline Bias & Authors' judgement & Support for judgement \\
\hline $\begin{array}{l}\text { Random sequence genera- } \\
\text { tion (selection bias) }\end{array}$ & Unclear risk & $\begin{array}{l}\text { Quote: "The randomised, examiner-blinded clinical trial..." However, method } \\
\text { of randomisation is not mentioned }\end{array}$ \\
\hline $\begin{array}{l}\text { Allocation concealment } \\
\text { (selection bias) }\end{array}$ & Unclear risk & Not mentioned \\
\hline $\begin{array}{l}\text { Blinding of participants } \\
\text { and personnel (perfor- } \\
\text { mance bias) }\end{array}$ & Low risk & $\begin{array}{l}\text { Quote: "... which had been packed into a blinded kit box labelled with a unique } \\
\text { subject number" }\end{array}$ \\
\hline $\begin{array}{l}\text { Blinding of outcome as- } \\
\text { sessment (detection bias) }\end{array}$ & Unclear risk & $\begin{array}{l}\text { Quote: "The randomised, examiner-blinded clinical trial..." However, method } \\
\text { of blinding is not mentioned }\end{array}$ \\
\end{tabular}


Gerlach 2004 (Continued)

All outcomes

\begin{tabular}{lll}
$\begin{array}{l}\text { Incomplete outcome data } \\
\text { (attrition bias) } \\
\text { All outcomes }\end{array}$ & Low risk & $\begin{array}{l}\text { Quote: "1 subject in each group did not return for the Day 22 visit" } \\
\text { Comment: plausible effect size (difference in means or standardised difference } \\
\text { in means) among missing outcomes not enough to have a clinically relevant } \\
\text { impact on observed effect size }\end{array}$ \\
\hline $\begin{array}{l}\text { Selective reporting (re- } \\
\text { porting bias) }\end{array}$ & Low risk & $\begin{array}{l}\text { All outcomes described were reported. Conclusions are in accordance with the } \\
\text { results }\end{array}$ \\
\hline \begin{tabular}{l} 
Other bias \\
\hline
\end{tabular} & Low risk & None \\
\hline
\end{tabular}

\section{Gerlach $2004 e$}

\section{Methods}

Title: placebo-controlled clinical trial evaluating a 10\% hydrogen peroxide whitening strip

Trial design: randomised, double-blinded, placebo-controlled trial

Location: University of Florida, USA

Language: English

Number of centres: 1

Recruitment period: not reported

Funding source: Procter \& Gamble

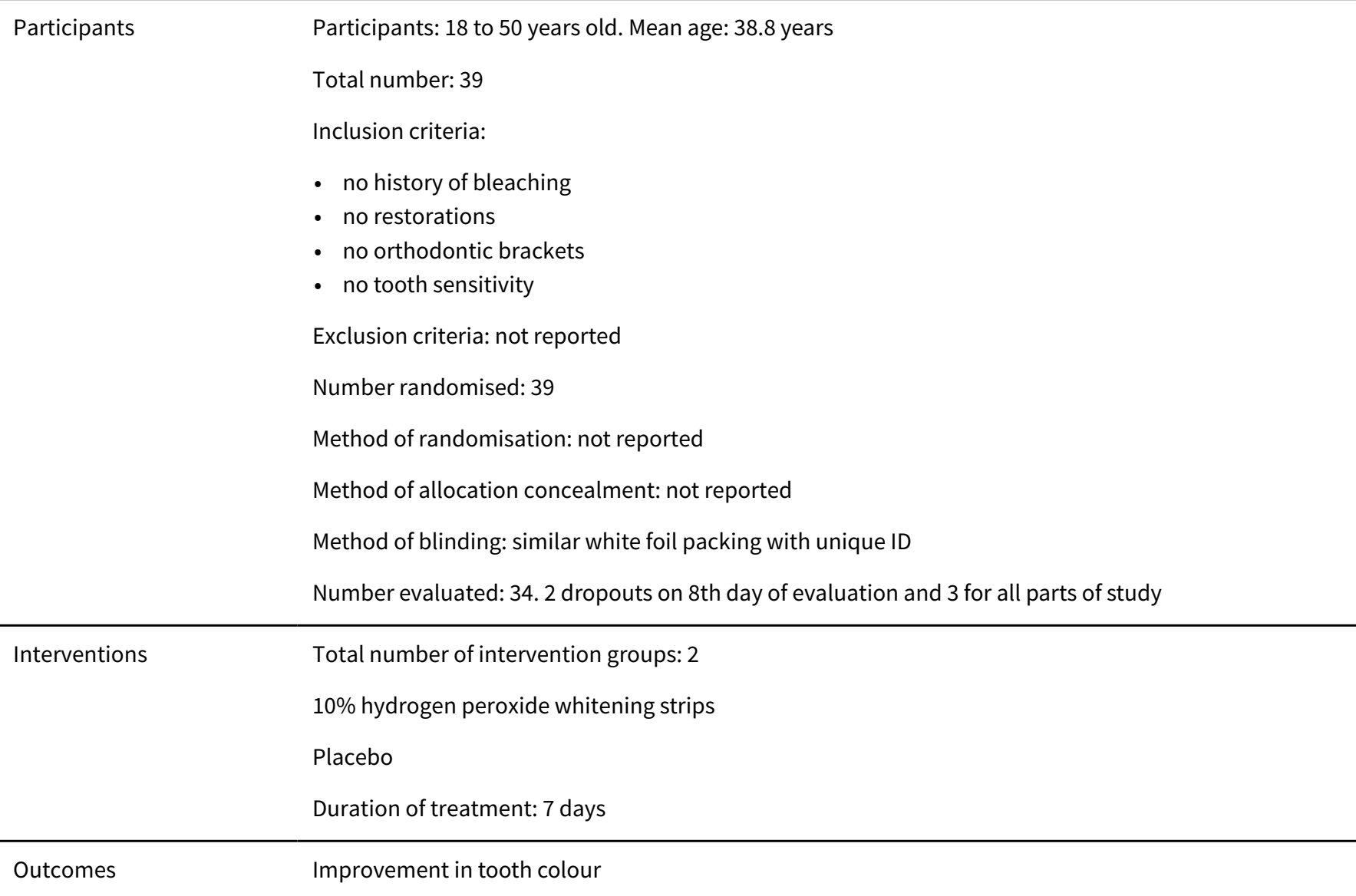


$b^{\star}$ : decreased $b^{\star}$ indicates reduced yellowness

$\Delta \mathrm{L}$ : increased $\Delta \mathrm{L}$ is increased brightness

Sotes
Adverse effects: oral irritation and tooth sensitivity
Health-related quality of life: not reported
Key conclusions of the study authors: "Statistically significant tooth whitening was evident after 3 days'
treatment with $10 \%$ hydrogen peroxide whitening strips, and colour improved with continued usage
over 7 days"
Contact: Dr Robert W Gerlach; gerlach.rw@pg.com

\section{Risk of bias}

\begin{tabular}{|c|c|c|}
\hline Bias & Authors' judgement & Support for judgement \\
\hline $\begin{array}{l}\text { Random sequence genera- } \\
\text { tion (selection bias) }\end{array}$ & Unclear risk & $\begin{array}{l}\text { Quote: "A randomised double-blinded, placebo-controlled clinical trial was } \\
\text { conducted... Groups were randomly assigned to treatment based on age and } \\
\text { baseline tooth colour." No further details are given }\end{array}$ \\
\hline $\begin{array}{l}\text { Allocation concealment } \\
\text { (selection bias) }\end{array}$ & Unclear risk & Not mentioned \\
\hline $\begin{array}{l}\text { Blinding of participants } \\
\text { and personnel (perfor- } \\
\text { mance bias) } \\
\text { All outcomes }\end{array}$ & Low risk & $\begin{array}{l}\text { Quote: "Each strip was packaged in an individual white foil pouch, with the } \\
\text { subject identification number... Strips were over packaged in a kit box,.." }\end{array}$ \\
\hline $\begin{array}{l}\text { Blinding of outcome as- } \\
\text { sessment (detection bias) } \\
\text { All outcomes }\end{array}$ & Unclear risk & Not mentioned \\
\hline $\begin{array}{l}\text { Incomplete outcome data } \\
\text { (attrition bias) } \\
\text { All outcomes }\end{array}$ & Low risk & $\begin{array}{l}\text { Quote: " } 3 \text { subjects ( } 1 \text { in the } 10 \% \text { group and } 2 \text { in placebo group) failed all or pat } \\
\text { of the day } 8 \text { evaluation" } \\
\text { Comment: plausible effect size (difference in means) among missing outcomes } \\
\text { not enough to have a clinically relevant impact on observed effect size }\end{array}$ \\
\hline $\begin{array}{l}\text { Selective reporting (re- } \\
\text { porting bias) }\end{array}$ & Low risk & $\begin{array}{l}\text { All outcomes mentioned are reported. Conclusions are in accordance with the } \\
\text { results }\end{array}$ \\
\hline Other bias & Low risk & None \\
\hline
\end{tabular}

Gerlach 2005

Methods Title: clinical trial comparing 2 hydrogen peroxide tooth whitening systems: strips versus pre-rinse

Trial design: randomised, examiner-blinded, parallel-group trial

Location: not reported

Language: English

Number of centres: 1 
Gerlach 2005 (Continued)

Recruitment period: not reported

Funding source: Procter \& Gamble

Participants: 29 to 58 years old. Mean age 39.8 years
Total number: 28
Inclusion criteria:
- 4 maxillary anterior teeth
- no sensitivity
Exclusion criteria: not reported
Number randomised: 28
Method of randomisation: not reported
Method of allocation concealment: not reported
Method of blinding: not reported
Number evaluated: 28

Interventions

Total number of intervention groups: 2

Mouthwash: $2 \%$ hydrogen peroxide

Strip: $10 \%$ hydrogen peroxide

Duration of treatment: 7 days

Improvement in tooth shade
$\mathrm{a}^{\star}, \mathrm{b}^{\star}$ and $\Delta \mathrm{L}$ were recorded
Whitening benefit was represented by negative $\mathrm{b}^{\star}$ (yellowness reduction), and positive $\Delta \mathrm{L}$ (increasing
lightness)

Notes

Sample size calculation: not reported

Adverse effects: tooth sensitivity and oral irritation

Health-related quality of life: not reported

Key conclusions of the study authors: "Relative to baseline, the whitening strip group experienced colour improvement at day 3 , continuing through day 8 . The pre-rinse group did not show any significant change at day 3 , and had a significant increase in yellowness at day 8 . The strip group exhibiting significantly greater whitening at day 8 . Both products were well tolerated, with no participants discontinuing treatment early as the result of an adverse event. In head-to-head testing, 7-day use of the $10 \%$ hydrogen peroxide whitening strips resulted in significant tooth colour improvement relative to a barrier-free $2 \%$ hydrogen peroxide pre-brushing mouthrinse"

Correspondence required: no

Contact: Dr Geralch; geralch.rw@pg.com

\section{Risk of bias}

\section{Bias}

Authors' judgement Support for judgement 
Gerlach 2005 (Continued)

$\begin{array}{ll}\begin{array}{l}\text { Random sequence genera- } \\ \text { tion (selection bias) }\end{array} & \text { Unclear risk } \\ \end{array}$
tion (selection bias) method of randomisation is not mentioned

Allocation concealment Unclear risk Not mentioned
(selection bias)

\begin{tabular}{|c|c|c|}
\hline $\begin{array}{l}\text { Blinding of participants } \\
\text { and personnel (perfor- }\end{array}$ & Unclear risk & $\begin{array}{l}\text { Quote: "Examiner-blinded, parallel-group, randomised controlled trial." How- } \\
\text { ever, method of blinding not mentioned }\end{array}$ \\
\hline
\end{tabular}

mance bias)

All outcomes

\begin{tabular}{lll}
\hline $\begin{array}{l}\text { Blinding of outcome as- } \\
\text { sessment (detection bias) } \\
\text { All outcomes }\end{array}$ & Unclear risk & $\begin{array}{l}\text { Quote: "Examiner-blinded, parallel-group, randomised controlled trial." How- } \\
\text { ever, method of blinding not mentioned }\end{array}$ \\
\hline $\begin{array}{l}\text { Incomplete outcome data } \\
\begin{array}{l}\text { (attrition bias) } \\
\text { All outcomes }\end{array}\end{array}$ & Low risk & Quote: "All the participants completed all the visits and were evaluated" \\
\hline $\begin{array}{l}\text { Selective reporting (re- } \\
\text { porting bias) }\end{array}$ & Low risk & $\begin{array}{l}\text { All outcomes described were reported. Conclusions are in accordance with the } \\
\text { results }\end{array}$ \\
\hline Other bias & Low risk & None \\
\hline
\end{tabular}

\section{Giniger 2005}

Methods

Title: a 180-day clinical investigation of the tooth whitening efficacy of a bleaching gel with added amorphous calcium phosphate

Trial design: double-blinded randomised controlled trial

Location: USA

Language: English

Number of centres: 1

Recruitment period: not reported

Funding source: Discus Dental, Culver City, USA

Participants

Participants: continuation of a previously published study, 27 agreed to continue. Mean age 46.4 years

Total number: 27

Inclusion criteria:

- absence of severe systemic diseases

- psychological diseases or both

- maxillary anterior tooth discolouration (equivalent to or darker than Vita shade A3)

- non-use of any dentist-supplied or -applied vital tooth bleaching treatment in the previous 6 months

- non-user of any in-office desensitising agent in the previous 6-month period

- no periodontal surgery or scaling performed in the previous 6 months

- patients with no carious, non-restored 6 max anterior teeth

Exclusion criteria: not reported

Number randomised: 27 
Giniger 2005 (Continued)

Method of randomisation: stratified randomisation schedule

Method of allocation concealment: secret coding

Method of blinding: identical pack

Number evaluated: 27

\begin{tabular}{l} 
Interventions \\
Control: $16 \%$ carbamide peroxide \\
Experimental: $16 \%$ carbamide peroxide with $0.5 \%$ soluble calcium phosphate derived in part from cal- \\
cium nitrate and potassium pyrophosphate \\
Duration of treatment: 19 days \\
\hline Improvement in tooth shade: Vita shade guide 11 (1) to B4 (16) \\
Gingival Index score: 0 (no gingivitis) to 3 (severe gingivitis) \\
Dentinal hypersensitivity: 0 (no pain) to 3 (pain during application of stimuli and immediately there af- \\
ter) \\
VAS scale: 0 (no pain) to 10 (highest level of pain) \\
Adverse effects: none \\
Key conclusions of the study authors: "This study demonstrated that the ACP product offers $10 \%$ bet- \\
ter long-term (6-month) whitening efficacy that the traditional bleaching gel. No significant adverse \\
effect. Tooth sensitivity, soft tissue health, and gingival health remained similar to baseline levels" \\
Contact: Dr Giniger; mginiger@mg-co.com
\end{tabular}

\section{Risk of bias}

\begin{tabular}{|c|c|c|}
\hline Bias & Authors' judgement & Support for judgement \\
\hline $\begin{array}{l}\text { Random sequence genera- } \\
\text { tion (selection bias) }\end{array}$ & Low risk & $\begin{array}{l}\text { Quote: "..we randomised subjects into test and control groups, according to a } \\
\text { stratified randomisation schedule" }\end{array}$ \\
\hline $\begin{array}{l}\text { Allocation concealment } \\
\text { (selection bias) }\end{array}$ & Low risk & $\begin{array}{l}\text { Quote: "The evaluator of the teeth shades and sensitivity scores (MG) was } \\
\text { blinded to this schedule.. other than a secret product code number known on- } \\
\text { ly to a co-worker" }\end{array}$ \\
\hline $\begin{array}{l}\text { Blinding of participants } \\
\text { and personnel (perfor- } \\
\text { mance bias) } \\
\text { All outcomes }\end{array}$ & Low risk & $\begin{array}{l}\text { Quote: "The evaluator of the teeth shades and sensitivity scores (MG) was } \\
\text { blinded to this schedule, and the products looked identical to him and to the } \\
\text { subjects" }\end{array}$ \\
\hline $\begin{array}{l}\text { Blinding of outcome as- } \\
\text { sessment (detection bias) } \\
\text { All outcomes }\end{array}$ & Low risk & $\begin{array}{l}\text { Quote: "The evaluator of the teeth shades and sensitivity scores (MG) was } \\
\text { blinded to this schedule, and the products looked identical to him and to the } \\
\text { subjects" }\end{array}$ \\
\hline $\begin{array}{l}\text { Incomplete outcome data } \\
\text { (attrition bias) } \\
\text { All outcomes }\end{array}$ & Low risk & Quote: "All 27 requalified subjects completed the study" \\
\hline $\begin{array}{l}\text { Selective reporting (re- } \\
\text { porting bias) }\end{array}$ & Low risk & $\begin{array}{l}\text { All outcomes described were reported. Conclusions are in accordance with the } \\
\text { results }\end{array}$ \\
\hline
\end{tabular}


Giniger 2005 (Continued)
Other bias
Low risk
None

Hannig 2007

Methods

Title: efficacy and tolerability of 2 home bleaching systems having different peroxide delivery

Trial design: randomised, single-blinded, parallel-group trial

Location: University of Göttingen, Germany

Language: English

Number of centres: 1

Recruitment period: not reported

Funding source: Procter \& Gamble

Participants

Participants: 18 to 60 years. Mean age 29.35 years

Total number: 47

Inclusion criteria:

- volunteers with resorted or caries-free teeth

- anterior tooth colour Vita shade A2 or darker

- with no crowns on upper cupids or incisors

Exclusion criteria:

- patients with prior tooth sensitivity

- restoration on anterior teeth

- poor oral hygiene

- generalized gingival recession

- caries

- heavy structural alteration of the tooth structure

- tetracycline or fluorosis staining

- patients with systemic disorders

Number randomised: 47

Method of randomisation: not reported

Method of allocation concealment: not reported

Method of blinding: not reported

Number evaluated: 43 (at 2 weeks)

Interventions

Total number of intervention groups: 2

$6 \%$ hydrogen peroxide strips

$10 \%$ carbamide peroxide gel in tray

Duration of treatment: 14 days 
Adverse effects: transient tooth sensitivity and oral soft tissue irritation

Health-related quality of life: not reported

Key conclusions of the study authors: "Both whitening systems showed significant tooth colour improvement after 2 weeks of use.... No statistical significant difference was observed between the 2 systems..... Both systems were well tolerated and caused comparable levels of transient tooth sensitivity and oral soft tissue irritation"

Correspondence required: no

Contact: Christian Hannig, christian.hannig@uniklinik-feriburg.de

\section{Risk of bias}

\section{Bias}

Random sequence genera- Unclear risk tion (selection bias)

\section{Authors' judgement Support for judgement}

Quote: "this single-blinded, randomised, parallel-group study...... Subjects were stratified according to the baseline anterior maxillary tooth brightness $\left(L^{\star}\right)$ as determined by digital image analysis system and by the criteria of smoker/non-smoker. Randomisation to treatment was performed within each strata." However, method not reported

Allocation concealment Unclear risk Not mentioned

(selection bias)

$\begin{array}{ll}\begin{array}{l}\text { Blinding of participants } \\ \text { and personnel (perfor- }\end{array} & \text { Unclear risk }\end{array}$
mance bias)

All outcomes

\begin{tabular}{|c|c|c|}
\hline $\begin{array}{l}\text { Blinding of outcome as- } \\
\text { sessment (detection bias) } \\
\text { All outcomes }\end{array}$ & Unclear risk & $\begin{array}{l}\text { Quote: "this single-blinded, randomised, parallel-group study." However, } \\
\text { method not reported }\end{array}$ \\
\hline $\begin{array}{l}\text { Incomplete outcome data } \\
\text { (attrition bias) } \\
\text { All outcomes }\end{array}$ & Low risk & $\begin{array}{l}\text { Quote: " } 5 \text { subjects dropped out of the study due to reasons not related to } \\
\text { bleaching therapy at different stages. } 2 \text { subjects from the WS [strips] group } \\
\text { withdrew after } 5 \text { days of treatment because of product-related side effects" }\end{array}$ \\
\hline & & $\begin{array}{l}\text { Comment: number of participants at } 2 \text { weeks: } 22 \text { in tray group and } 21 \text { in strip } \\
\text { group. Missing outcome data balanced in numbers across intervention groups }\end{array}$ \\
\hline $\begin{array}{l}\text { Selective reporting (re- } \\
\text { porting bias) }\end{array}$ & Low risk & $\begin{array}{l}\text { All outcomes described were reported. Conclusions are in accordance with the } \\
\text { results }\end{array}$ \\
\hline Other bias & Low risk & None \\
\hline
\end{tabular}

\section{Hasturk 2004}

Methods

Title: efficacy of a fluoridated hydrogen peroxide-based mouthrinse for the treatment of gingivitis

Trial design: randomised, double-blinded, placebo-controlled, parallel-group trial

Location: Boston Medical Center, USA 
Hasturk 2004 (Continued)
Language: English
Number of centres: 1
Recruitment period: not reported
Funding source: Dent Mat

Participants: 18 to 50 years old
Total number: 110
Inclusion criteria:
- had a minimum 20 natural teeth excluding wisdom teeth
- no periodontitis or history of periodontitis
- had natural anterior maxillary teeth with no restorations
Exclusion criteria:
- subjects who had orthodontic appliances
- tumour in the oral cavity
- carious lesions requiring immediate treatment
- subjects who received antibiotic therapy in the 30 days before the study begins
- subjects who were on long-term anti-inflammatory therapy
Number randomised: 99
Method of randomisation: random numbers chart
Method of allocation concealment: reported
Method of blinding: similar coded bottle
Number evaluated: 78

Interventions

Total number of intervention groups: 2

Hydrogen peroxide $+0.05 \%$ sodium fluoride mouthrinse

Placebo

Duration of treatment: 30 seconds twice daily for 6 months

\begin{tabular}{ll}
\hline Outcomes & Plaque Index \\
Modified Gingival Index \\
Bleeding on probing dichotomous (1 or 0) \\
Intensity of stain: dichotomous ( 1 if lightened or if no change) \\
Sample size calculation: not reported \\
Adverse effects: not reported \\
Health-related quality of life: not reported \\
Key conclusions of the study authors: "Subjects using the test rinse were also 6 times more likely to ex- \\
hibit an improvement in tooth colour after 6 months than were subjects using placebo. As a result of \\
the clinical evaluations and microbial analysis, test mouthrinse was found to be safe during a 6 -month \\
period"
\end{tabular}


Hasturk 2004 (Continued)

Contact: Thomas E Van Dyke, tvandyke@bu.edu

\section{Risk of bias}

\begin{tabular}{lll}
\hline Bias & Authors' judgement & Support for judgement \\
\hline $\begin{array}{l}\text { Random sequence genera- } \\
\text { tion (selection bias) }\end{array}$ & Low risk & $\begin{array}{l}\text { Quote: "Treatment was assigned by use of random number charts, and subject } \\
\text { assignment was made by an individual who was not involved in the treatment } \\
\text { or measurement procedures" }\end{array}$ \\
\hline $\begin{array}{l}\text { Allocation concealment } \\
\text { (selection bias) }\end{array}$ & Low risk & $\begin{array}{l}\text { Quote: "Treatment was assigned by use of random number charts, and subject } \\
\text { assignment was made by an individual who was not involved in the treatment } \\
\text { or measurement procedures" }\end{array}$ \\
\hline
\end{tabular}

\begin{tabular}{|c|c|c|}
\hline $\begin{array}{l}\text { Blinding of participants } \\
\text { and personnel (perfor- } \\
\text { mance bias) } \\
\text { All outcomes }\end{array}$ & Low risk & $\begin{array}{l}\text { Quotes: "The placebo rinse was an identical base formulation to the test } \\
\text { mouthrinse...;" " Mouthrinses were labelled to conform with prescribing regu- } \\
\text { lations and were coded to maintain double masking ..." }\end{array}$ \\
\hline $\begin{array}{l}\text { Blinding of outcome as- } \\
\text { sessment (detection bias) } \\
\text { All outcomes }\end{array}$ & Low risk & $\begin{array}{l}\text { Quote: "All clinical measurements were performed under the same conditions } \\
\text { by the same investigator who was blinded to the treatment" }\end{array}$ \\
\hline $\begin{array}{l}\text { Incomplete outcome data } \\
\text { (attrition bias) } \\
\text { All outcomes }\end{array}$ & Unclear risk & $\begin{array}{l}\text { Quotes: "Each subject was considered as the unit of analysis. Therefore, sub- } \\
\text { ject who completed at least } 1 \text { post-baseline clinical assessment visit were } \\
\text { included in the intent-to-treat population;" " } 99 \text { subjects were randomised, } \\
\text { and } 78 \text { subjects completed the whole course of the study. } 38 \text { subjects were in } \\
\text { placebo group and } 40 \text { in the test group" } \\
\text { Comment: no details of reasons for dropouts are given }\end{array}$ \\
\hline $\begin{array}{l}\text { Selective reporting (re- } \\
\text { porting bias) }\end{array}$ & Low risk & $\begin{array}{l}\text { All outcomes have been reported adequately. Conclusions conform to the re- } \\
\text { sults obtained }\end{array}$ \\
\hline Other bias & Low risk & None \\
\hline
\end{tabular}

\section{Hyland 2015}

\section{Methods}

Title: a new 3-component formulation for the efficient whitening of teeth (Carbamide Plus)

Trial design: randomised, double-blinded, placebo-controlled, clinical trial

Location: Eastman Dental Hospital, UK

Language: English

Number of centres: 1

Recruitment period: not reported

Funding source: SMT Ltd and DEL CAST studentship

$\begin{array}{ll}\text { Participants } & \text { Participants: adults } \\ & \text { Total number: } 33 \\ & \text { Inclusion criteria: not reported } \\ & \text { Exclusion criteria: }\end{array}$


Hyland 2015 (Continued)

- heavily restored upper left central incisor or upper right canine

- pregnancy or breastfeeding

- previously undergone a course of vital tooth whitening

- smokers

- active dental disease

- severe dentine hypersensitivity

- uncontrolled dental disease

- unable to attend on data collection

Number randomised: 32

Method of randomisation: not reported

Method of allocation concealment: not reported

Method of blinding: not reported

Number evaluated: 32

Interventions

Total number of intervention groups: 3

$5 \%$ carbamide peroxide gel

$10 \%$ carbamide peroxide gel

Placebo

Duration of treatment: 2 hours per day for 2 weeks

\section{Outcomes}

Improvement in tooth colour

$\Delta \mathrm{L}, \mathrm{a}^{\star}, \mathrm{b}^{\star}$ : increase in $\Delta \mathrm{L}$ and reduction in $\mathrm{b}^{\star}$ indicates whitening

Sample size calculation: not reported
Adverse effects: not reported
Health-related quality of life: not reported
Key conclusions of the study authors: "A new tooth-whitening product Carbamide Plus containing urea,
hydrogen peroxide, and STPP as active components containing 5\% hydrogen peroxide has been shown
to be as effective as the commercially available carbamide peroxide containing $10 \%$ hydrogen perox-
ide. There were no statistically significant differences between Carbamide Plus and $10 \%$ carbamide
peroxide in tooth whitening at 2 weeks following daily wear of tooth whitening trays for 2 hours per
day"
Correspondence required: no
Contact: BW Hyland; j.callan@ulster.ac.uk

Risk of bias

\begin{tabular}{lll}
\hline Bias & Authors' judgement & Support for judgement \\
\hline $\begin{array}{l}\text { Random sequence genera- } \\
\text { tion (selection bias) }\end{array}$ & Unclear risk & $\begin{array}{l}\text { Quote: "The recruited subjects were randomly allocated to } 1 \text { of } 3 \text { study group- } \\
\text { ings: non-active placebo gel..." However, method is not reported }\end{array}$ \\
\hline $\begin{array}{l}\text { Allocation concealment } \\
\text { (selection bias) }\end{array}$ & Unclear risk & Not mentioned \\
\hline
\end{tabular}


Hyland 2015 (Continued)

Blinding of participants Unclear risk Quote: "randomised, double-blinded, placebo-controlled clinical trial." Howand personnel (perfor-

mance bias)

All outcomes

\section{ever, method is not reported}

\section{Blinding of outcome as- Unclear risk} sessment (detection bias)

All outcomes

\begin{tabular}{lll}
\hline $\begin{array}{l}\text { Incomplete outcome data } \\
\text { (attrition bias) } \\
\text { All outcomes }\end{array}$ & Low risk & No dropouts \\
\hline $\begin{array}{l}\text { Selective reporting (re- } \\
\text { porting bias) }\end{array}$ & Low risk & $\begin{array}{l}\text { All outcomes described were reported. Conclusions are in accordance with the } \\
\text { results }\end{array}$ \\
\hline Other bias & Low risk & None \\
\hline
\end{tabular}

\section{Karpinia 2002}

\begin{tabular}{|c|c|}
\hline \multirow[t]{7}{*}{ Methods } & Title: vital bleaching with 2 at-home professional systems \\
\hline & Trial design: randomised controlled trial \\
\hline & Location: not reported \\
\hline & Language: English \\
\hline & Number of centres: 1 \\
\hline & Recruitment period: not reported \\
\hline & Funding source: Procter \& Gamble \\
\hline \multirow[t]{13}{*}{ Participants } & Participants: 18 to 65 years old. Mean age 37.2 years \\
\hline & Total number: 69 \\
\hline & Inclusion criteria: \\
\hline & - 18 years and above \\
\hline & - 4 maxillary anterior teeth with shade greater than $\mathrm{A} 2$ \\
\hline & Exclusion criteria: \\
\hline & - previous history of bleaching \\
\hline & - sensitivity of the tooth \\
\hline & Number randomised: 69 \\
\hline & Method of randomisation: randomised in blocks of 4 \\
\hline & Method of allocation concealment: not reported \\
\hline & Method of blinding: unique coded label \\
\hline & Number evaluated: 67 \\
\hline
\end{tabular}


Karpinia 2002 (Continued)

\section{$6.5 \%$ hydrogen peroxide strips}

$10 \%$ carbamide peroxide in tray

Duration of treatment: 30 minutes twice daily for 3 weeks

Outcomes Improvement in tooth shade: $\Delta \mathrm{L}, \mathrm{a}^{\star}, \mathrm{b}^{\star}$ values were recorded. Increase in $\Delta \mathrm{L}$ and decrease in $\mathrm{b}^{\star}$ indi-
cates whitening

Sample size calculation: not reported
Adverse effects: oral and gingival irritation
Health-related quality of life: not reported
Key conclusions of the study authors: "For between group comparisons, strip subjects had a statistical-
ly significant or directionally favourable whitening response relative to the tray system at intermediary
time points, while at the end of treatment, the strip group had highly statistically significant (P< or =
0.005), superior whitening response for all colour parameters measured in the study. Both treatments
were generally well tolerated, with $35 \%$ to $40 \%$ of the subjects in each group reporting minor tooth sen-
sitivity or gingival irritation"
Correspondence required: no
Contact: KA Karpinia, University of Florida, Gainesville, USA

\section{Risk of bias}

\begin{tabular}{|c|c|c|}
\hline Bias & Authors' judgement & Support for judgement \\
\hline $\begin{array}{l}\text { Random sequence genera- } \\
\text { tion (selection bias) }\end{array}$ & Low risk & Quote: "The study population was randomised in blocks of 4" \\
\hline $\begin{array}{l}\text { Allocation concealment } \\
\text { (selection bias) }\end{array}$ & Unclear risk & Not mentioned \\
\hline $\begin{array}{l}\text { Blinding of participants } \\
\text { and personnel (perfor- } \\
\text { mance bias) } \\
\text { All outcomes }\end{array}$ & Low risk & $\begin{array}{l}\text { Quote: "Each product was over labelled with a unique subject identification } \\
\text { number" }\end{array}$ \\
\hline $\begin{array}{l}\text { Blinding of outcome as- } \\
\text { sessment (detection bias) } \\
\text { All outcomes }\end{array}$ & Unclear risk & Not mentioned \\
\hline $\begin{array}{l}\text { Incomplete outcome data } \\
\text { (attrition bias) } \\
\text { All outcomes }\end{array}$ & Low risk & $\begin{array}{l}\text { Quote: "2 patients from tray group and } 1 \text { patient from tray and strip group } \\
\text { missed the evaluation group at } 7 \text { days and } 3 \text { weeks respectively" } \\
\text { Comment: missing outcome data balanced in numbers across intervention } \\
\text { groups }\end{array}$ \\
\hline $\begin{array}{l}\text { Selective reporting (re- } \\
\text { porting bias) }\end{array}$ & Low risk & $\begin{array}{l}\text { All outcomes described were reported. Conclusions are in accordance with the } \\
\text { results }\end{array}$ \\
\hline Other bias & Low risk & None \\
\hline
\end{tabular}


Trial design: randomised, double-blinded study

Location: University of Maryland Dental School, USA

Language: English

Number of centres: 1

Recruitment period: not reported

Funding source: Dentsply

Participants
Total number: 57
Inclusion criteria: maxillary anterior teeth of shade 18 to 65 years old
Exclusion criteria: not reported
Number randomised: 56
Method of randomisation: reported
Method of allocation concealment: not reported
Method of blinding: not reported
Number evaluated: 52

Interventions

Total number of intervention groups: 2

Experiment: 15\% carbamide peroxide gel

Control: $10 \%$ carbamide peroxide gel

Duration of treatment: 2 weeks

\begin{tabular}{ll}
\hline Outcomes & $\begin{array}{l}\text { Improvement in tooth colour } \\
\text { Sensitivity: } 0 \text { (no pain) to } 20 \text { (severe) }\end{array}$ \\
\hline Notes & Sample size calculation: not reported \\
Adverse effects: sensitivity & Health-related quality of life: not reported \\
& Key conclusions of the study authors: "There was a significant difference in colour change between the \\
$10 \%$ CP and $15 \%$ CP groups at the end of the study period. There was no significant difference in level \\
of tooth sensitivity between the 2 groups, and the incidence was equal; there was, however, a signifi- \\
cant difference in variability of tooth sensitivity between the 2 groups" \\
Correspondence required: no \\
Contact: Patricia W Kihn; tkihn@caulk.com
\end{tabular}

\section{Risk of bias}


Kihn 2000 (Continued) $\begin{array}{ll}\begin{array}{l}\text { Random sequence genera- } \\ \text { tion (selection bias) }\end{array} & \text { Low risk } \\ \end{array}$

\begin{tabular}{|c|c|c|}
\hline $\begin{array}{l}\text { Allocation concealment } \\
\text { (selection bias) }\end{array}$ & Unclear risk & Not mentioned \\
\hline $\begin{array}{l}\text { Blinding of participants } \\
\text { and personnel (perfor- } \\
\text { mance bias) } \\
\text { All outcomes }\end{array}$ & Unclear risk & $\begin{array}{l}\text { Quote: "authors conducted a double-blinded study of human subjects to..." } \\
\text { However, method of blinding is not reported }\end{array}$ \\
\hline $\begin{array}{l}\text { Blinding of outcome as- } \\
\text { sessment (detection bias) } \\
\text { All outcomes }\end{array}$ & Unclear risk & $\begin{array}{l}\text { Quote: "authors conducted a double-blinded study of human subjects to..." } \\
\text { However, method of blinding is not reported }\end{array}$ \\
\hline $\begin{array}{l}\text { Incomplete outcome data } \\
\text { (attrition bias) } \\
\text { All outcomes }\end{array}$ & Low risk & $\begin{array}{l}\text { Quote: "Of the } 56 \text { subjects who began the study, } 26 \text { pairs of matched subjects } \\
\text { ( } n=52 \text { individual subjects) completed the study" Commnet: missing outcome } \\
\text { data balanced in numbers across intervention groups }\end{array}$ \\
\hline $\begin{array}{l}\text { Selective reporting (re- } \\
\text { porting bias) }\end{array}$ & Low risk & $\begin{array}{l}\text { All outcomes described were reported. Conclusions are in accordance with the } \\
\text { results }\end{array}$ \\
\hline Other bias & Low risk & None \\
\hline
\end{tabular}

\title{
Kose 2011
}

Methods

Title: clinical effects of at-home bleaching along with desensitizing agent application

Trial design: randomised, double-blinded, controlled trial

Location: University Estadua, Brazil

Language: English

Number of centres: 1

Recruitment period: not reported

Funding source: FGM Dental Products

Participants

\author{
Participants: 18 to 30 years old. Mean age 24 years \\ Total number: 60 \\ Inclusion criteria: \\ - 18 and older \\ - 6 caries and restoration free teeth \\ - A2 shade or darker \\ Exclusion criteria: \\ - previous vital bleaching, apparent caries, periodontal disease \\ - orthodontic appliances, anterior restorations \\ - history of dentine hypersensitivity \\ - bruxism \\ Number randomised: 60
}


Kose 2011 (Continued)

Method of randomisation: coin toss

Method of allocation concealment: not reported

Method of blinding: similar syringes

Number evaluated: 60

\begin{tabular}{ll}
\hline Interventions & Total number of intervention groups: 2 \\
& $16 \%$ carbamide peroxide in tray + placebo \\
& $16 \%$ carbamide peroxide in tray + desensitizer gel (DG; $5 \%$ potassium nitrate and $2 \%$ sodium fluoride) \\
& Duration of treatment: 4 weeks
\end{tabular}

Outcomes Improvement in tooth shade: Vita shade guide B1 (highest) to C4 (lowest)

Tooth sensitivity: 0 - none, 1 - mild, 2 - moderate, 3 - considerable, 4 - severe

Notes

Sample size calculation: not reported

Adverse effects: tooth sensitivity

Health-related quality of life: not reported

Key conclusions of the study authors: "The use of desensitizing agent did not affect the bleaching efficacy of the CP. The prevalence and intensity of tooth sensitivity was similar for both groups. However, participants from the placebo group had sensitivity in $33.6 \%$ of the bleaching days, which was significantly higher than the desensitizing agent experimental group (20.1\%)"

Correspondence required: no

Contact: Dr Kose, School of Dentistry, State University of Ponda, Santa Catarina, Brazil

\section{Risk of bias}

\begin{tabular}{|c|c|c|}
\hline Bias & Authors' judgement & Support for judgement \\
\hline $\begin{array}{l}\text { Random sequence genera- } \\
\text { tion (selection bias) }\end{array}$ & Low risk & $\begin{array}{l}\text { Quote: "subjects were randomly divided into experimental or control group by } \\
\text { coin toss" }\end{array}$ \\
\hline $\begin{array}{l}\text { Allocation concealment } \\
\text { (selection bias) }\end{array}$ & Unclear risk & Not mentioned \\
\hline $\begin{array}{l}\text { Blinding of participants } \\
\text { and personnel (perfor- } \\
\text { mance bias) } \\
\text { All outcomes }\end{array}$ & Low risk & $\begin{array}{l}\text { Quote: "Neither the subjects nor the evaluators knew to which group the sub- } \\
\text { jects were assigned" }\end{array}$ \\
\hline $\begin{array}{l}\text { Blinding of outcome as- } \\
\text { sessment (detection bias) } \\
\text { All outcomes }\end{array}$ & Low risk & $\begin{array}{l}\text { Quote: "Neither the subjects nor the evaluators knew to which group the sub- } \\
\text { jects were assigned" }\end{array}$ \\
\hline $\begin{array}{l}\text { Incomplete outcome data } \\
\text { (attrition bias) } \\
\text { All outcomes }\end{array}$ & Low risk & Quote: "All 60 participants who began study completed the study" \\
\hline $\begin{array}{l}\text { Selective reporting (re- } \\
\text { porting bias) }\end{array}$ & Low risk & $\begin{array}{l}\text { All outcomes described were reported. Conclusions are in accordance with the } \\
\text { results }\end{array}$ \\
\hline
\end{tabular}


Kose 2011 (Continued)
Other bias
Low risk
None

Kowitz 1994

$\begin{array}{ll}\text { Methods } & \text { Title: comparative clinical evaluation of } 2 \text { pro } \\ \text { Trial design: single-blinded, parallel, randomis } & \text { Location: not reported } \\ \text { Language: } \text { English } & \text { Number of centres: } 1 \\ \text { Recruitment period: not reported } & \text { Funding source: not reported } \\ & \text { Participants: adults } \\ & \text { Total number: } 50 \\ \text { Inclusion criteria: } \text { not reported } & \text { Exclusion criteria: not reported } \\ \text { Number randomised: } 50 \\ \text { Method of randomisation: not reported } \\ \text { Method of allocation concealment: reported } \\ \text { Method of blinding: coded packs } \\ \text { Number evaluated: } 48\end{array}$

\begin{tabular}{ll}
\hline Interventions & Total number of intervention groups: 2 \\
$10 \%$ urea peroxide from 2 different brands 1 hour application twice daily \\
Duration of treatment: 2 weeks \\
\hline Improvement in tooth shade \\
Sensitivity \\
Patient-reported satisfaction \\
Sample size calculation: not reported \\
Adverse effects: sensitivity \\
Health-related quality of life: not reported \\
Key conclusions of the study authors: "Colgate Platinum was 62\% more effective at tooth whitening af- \\
ter 1 week and $83 \%$ more effective after 2 weeks of treatment versus Rembrandt. At the termination of \\
the study, the mean colour difference (deltaE) for Colgate Platinum was 4.29 and 2.34 for Rembrandt. \\
Statistical analysis demonstrated that the Colgate product is significantly superior at increasing tooth \\
whiteness, increasing tooth lightness, reducing redness, and reducing yellowness. In this study, no ad- \\
verse reactions were noted on clinical examination and none were reported by panelists with normal \\
healthy dentition"
\end{tabular}


Kowitz 1994 (Continued)

Correspondence required: no

Contact: Dr Kowitz, University of California, USA

\section{Risk of bias}

\begin{tabular}{|c|c|c|}
\hline Bias & Authors' judgement & Support for judgement \\
\hline $\begin{array}{l}\text { Random sequence genera- } \\
\text { tion (selection bias) }\end{array}$ & Unclear risk & $\begin{array}{l}\text { Quote: "single-blinded, randomised, 2-celled parallel clinical trial." However, } \\
\text { method of randomisation is not reported }\end{array}$ \\
\hline $\begin{array}{l}\text { Allocation concealment } \\
\text { (selection bias) }\end{array}$ & Low risk & $\begin{array}{l}\text { Quote: "Neither the subject no the investigator were informed to which group } \\
\text { the individual belonged" }\end{array}$ \\
\hline $\begin{array}{l}\text { Blinding of participants } \\
\text { and personnel (perfor- } \\
\text { mance bias) } \\
\text { All outcomes }\end{array}$ & Low risk & Quote: "Each subject was assigned a coded test product...." \\
\hline $\begin{array}{l}\text { Blinding of outcome as- } \\
\text { sessment (detection bias) } \\
\text { All outcomes }\end{array}$ & Unclear risk & Not reported \\
\hline $\begin{array}{l}\text { Incomplete outcome data } \\
\text { (attrition bias) } \\
\text { All outcomes }\end{array}$ & Low risk & $\begin{array}{l}\text { Quote: "2 dropped out because of problems unrelated to study" } \\
\text { Comment: missing outcome data balanced in numbers across intervention } \\
\text { groups }\end{array}$ \\
\hline $\begin{array}{l}\text { Selective reporting (re- } \\
\text { porting bias) }\end{array}$ & Low risk & $\begin{array}{l}\text { All outcomes described were reported. Conclusions are in accordance with the } \\
\text { results }\end{array}$ \\
\hline Other bias & Low risk & None \\
\hline
\end{tabular}

Krause 2008

Title: subjective intensities of pain and contentment with treatment outcomes during tray bleaching of
vital teeth employing different carbamide peroxide concentrations
Trial design: double-blinded, randomised controlled trial
Location: university
Language: English
Number of centres: 1
Recruitment period: not reported
Funding source: not reported

Participants

Participants: mean age 31 years

Total number: 30

Inclusion criteria:

- patients with teeth not discoloured for extrinsic or intrinsic reasons

- non-smokers 
Krause 2008 (Continued)

Exclusion criteria: not reported

Number randomised: 30

Method of randomisation: computer generated numbers

Method of allocation concealment: not reported

Method of blinding: unlabelled similar packs

Number evaluated: 30

\begin{tabular}{ll}
\hline Interventions & Total number of intervention groups: 3 \\
$10 \%$ carbamide peroxide tray \\
$17 \%$ carbamide peroxide tray \\
$0 \%$ carbamide peroxide tray \\
Duration of treatment: 7 days \\
Pain sensation \\
Patient contentment with bleaching: 0 no contentment, 100 maximum contentment \\
Sample size calculation: not reported \\
Adverse effects: not reported \\
Health-related quality of life: yes, patient satisfaction \\
Key conclusions of the study authors: "Application of carbamide peroxide-containing bleaching agents \\
to vital teeth causes pain correlated with the \\
$\begin{array}{l}\text { agent's concentration. Since both highly and less concentrated gels might result in a similar content- } \\
\text { ment with the treatment outcome, the use of highly concentrated agents appears not to be justified to } \\
\text { improve vital tooth colour" } \\
\text { Contact: Felix Krause, fkrause@uni-bonn.de }\end{array}$
\end{tabular}

\section{Risk of bias}

\begin{tabular}{|c|c|c|}
\hline Bias & Authors' judgement & Support for judgement \\
\hline $\begin{array}{l}\text { Random sequence genera- } \\
\text { tion (selection bias) }\end{array}$ & Low risk & $\begin{array}{l}\text { Quote: "kits were assigned to the patients employing computer-generated } \\
\text { random numbers by a different operator" }\end{array}$ \\
\hline $\begin{array}{l}\text { Allocation concealment } \\
\text { (selection bias) }\end{array}$ & Unclear risk & Not mentioned \\
\hline $\begin{array}{l}\text { Blinding of participants } \\
\text { and personnel (perfor- } \\
\text { mance bias) } \\
\text { All outcomes }\end{array}$ & Low risk & $\begin{array}{l}\text { Quote: "Unlabelled treatment kits numbered consecutively. Thus, both the op- } \\
\text { erator and the patient were unaware of the..." }\end{array}$ \\
\hline $\begin{array}{l}\text { Blinding of outcome as- } \\
\text { sessment (detection bias) } \\
\text { All outcomes }\end{array}$ & Low risk & $\begin{array}{l}\text { Quote: "Unlabelled treatment kits numbered consecutively. Thus, both the op- } \\
\text { erator and the patient were unaware of the..." }\end{array}$ \\
\hline $\begin{array}{l}\text { Incomplete outcome data } \\
\text { (attrition bias) }\end{array}$ & Low risk & All participants completed the study \\
\hline
\end{tabular}


Krause 2008 (Continued)

All outcomes

$\begin{array}{lll}\begin{array}{l}\text { Selective reporting (re- } \\ \text { porting bias) }\end{array} & \text { Low risk } & \begin{array}{l}\text { All outcomes described were reported. Conclusions are in accordance with the } \\ \text { results }\end{array}\end{array}$

Other bias Low risk None

Kugel 2000

Methods

Title: tooth-whitening efficacy and safety: a randomised and controlled clinical trial

Trial design: randomised, single-centre, double-blinded, parallel-group, placebo-controlled trial

Location: not reported

Language: English

Number of centres: 1

Recruitment period: not reported

Funding source: Procter \& Gamble

Participants

Participants: 18 to 66 years old. Mean age 33 years

Total number: 70

Inclusion criteria:

- 16 natural teeth and minimum 4 anterior teeth

- A2 shade or darker

Exclusion criteria:

- periodontal disease

- tetracycline stains

- restoration

- dental caries

- dental hypersensitivity

- previous history of bleaching

Number randomised: 70

Method of randomisation: block randomisation

Method of allocation concealment: not reported

Method of blinding: identical package and examiners were blinded

Number evaluated: 66.4 dropouts

Interventions

Total number of intervention groups: 2

$5.3 \%$ hydrogen peroxide gel delivered with polyethylene gel

Placebo

Duration of treatment: 2 weeks 
Kugel 2000 (Continued)

Oral soft and hard tissue examination

Dental hypersensitivity

Gingival Index: Loë and Silness Index

Plaque Index: Silness and Loë Index

Sample size calculation: not reported
Adverse effects: soft tissue irritation and dental hypersensitivity
Health-related quality of life: not reported
Key conclusions of the study: "Peroxide gel-treated group showed significant whitening of teeth. Both
treatments were generally well tolerated. The strips offer ease of use, comfort, and shorter duration of
wear compared with other at-home bleaching systems"

\section{Risk of bias}

\begin{tabular}{|c|c|c|}
\hline Bias & Authors' judgement & Support for judgement \\
\hline $\begin{array}{l}\text { Random sequence genera- } \\
\text { tion (selection bias) }\end{array}$ & Low risk & $\begin{array}{l}\text { Quote: "The study was a randomised, placebo-controlled, double-blinded, } \\
\text { parallel-group study. Block randomisation was done" }\end{array}$ \\
\hline $\begin{array}{l}\text { Allocation concealment } \\
\text { (selection bias) }\end{array}$ & Unclear risk & Not mentioned \\
\hline $\begin{array}{l}\text { Blinding of participants } \\
\text { and personnel (perfor- } \\
\text { mance bias) } \\
\text { All outcomes }\end{array}$ & Low risk & $\begin{array}{l}\text { Quote: "Except for the presence of peroxide, test products were identical in } \\
\text { composition and packaging" }\end{array}$ \\
\hline $\begin{array}{l}\text { Blinding of outcome as- } \\
\text { sessment (detection bias) } \\
\text { All outcomes }\end{array}$ & Low risk & Quote: "All examinations were performed blinded as to treatment assignment" \\
\hline $\begin{array}{l}\text { Incomplete outcome data } \\
\text { (attrition bias) } \\
\text { All outcomes }\end{array}$ & Low risk & $\begin{array}{l}2 \text { dropouts in each group at the end of the } 2 \text {-week trial. It is unlikely that } 4 \\
\text { dropouts could affect the overall results. Missing outcome data balanced in } \\
\text { numbers across intervention groups }\end{array}$ \\
\hline $\begin{array}{l}\text { Selective reporting (re- } \\
\text { porting bias) }\end{array}$ & Low risk & $\begin{array}{l}\text { All outcomes discussed were reported. Conclusions are in accordance with the } \\
\text { results }\end{array}$ \\
\hline Other bias & Low risk & None \\
\hline
\end{tabular}

\section{Kugel 2002}

\section{Methods}

Title: daily use of whitening strips on tetracycline-stained teeth: comparative results after 2 months

Trial design: randomised controlled trial

Location: Tufts University, USA

Language: English

Number of centres: 1

Recruitment period: not reported 
Kugel 2002 (Continued)

Funding source: Procter \& Gamble

\begin{tabular}{|c|c|}
\hline \multirow[t]{9}{*}{ Participants } & Participants: 22 to 70 years old \\
\hline & Total number: 40 \\
\hline & Inclusion criteria: adult patients with tetracycline stains \\
\hline & Exclusion criteria: not reported \\
\hline & Number randomised: 40 \\
\hline & Method of randomisation: not reported \\
\hline & Method of allocation concealment: not reported \\
\hline & Method of blinding: identical packages \\
\hline & Number evaluated: 33 \\
\hline \multirow[t]{4}{*}{ Interventions } & Total number of intervention groups: 2 \\
\hline & $6.5 \%$ hydrogen peroxide strips \\
\hline & $10 \%$ carbamide peroxide tray \\
\hline & Duration of treatment: 2 months \\
\hline \multirow[t]{2}{*}{ Outcomes } & Improvement with tooth shade. Vita shade guide arranged from darkest to lightest ( $B 4$ to $C 1$ ) \\
\hline & Plus 2 additional shades of $\mathrm{B} 4+$ and $\mathrm{C} 1+$ \\
\hline \multirow[t]{5}{*}{ Notes } & Sample size calculation: not reported \\
\hline & Adverse effects: tooth sensitivity and oral irritation \\
\hline & Health-related quality of life: not reported \\
\hline & $\begin{array}{l}\text { Key conclusions of the study authors: " } 6.5 \% \text { carbamide peroxide strips provided similar benefit to } 10 \% \\
\text { carbamide peroxide used over } 2 \text { months period" }\end{array}$ \\
\hline & Correspondence required: no \\
\hline
\end{tabular}

\section{Risk of bias}

\begin{tabular}{lll}
\hline Bias & Authors' judgement & Support for judgement \\
\hline $\begin{array}{l}\text { Random sequence genera- } \\
\text { tion (selection bias) }\end{array}$ & Unclear risk & $\begin{array}{l}\text { Quote: "randomised clinical trial." However, method of randomisation is not } \\
\text { reported }\end{array}$ \\
\hline $\begin{array}{l}\text { Allocation concealment } \\
\text { (selection bias) }\end{array}$ & Unclear risk & Not mentioned \\
\hline $\begin{array}{l}\text { Blinding of participants } \\
\text { and personnel (perfor- } \\
\text { mance bias) }\end{array}$ & Low risk & $\begin{array}{l}\text { Quote: "Products were packed in } 1 \text { month kits, and all labelling was identical } \\
\text { All outcomes }\end{array}$ \\
\hline
\end{tabular}

$\begin{array}{lll}\text { Blinding of outcome as- } & \text { Unclear risk Not mentioned } \\ \text { sessment (detection bias) } & \\ \text { All outcomes } & \end{array}$


Kugel 2002 (Continued)

\begin{tabular}{lll}
$\begin{array}{l}\text { Incomplete outcome data } \\
\text { (attrition bias) } \\
\text { All outcomes }\end{array}$ & Low risk & $\begin{array}{l}\text { Quote: "7 subjects withdrew from the treatment in the first month" } \\
\text { Comment: missing outcome data balanced in numbers across intervention } \\
\text { groups }\end{array}$ \\
\hline $\begin{array}{l}\text { Selective reporting (re- } \\
\text { porting bias) }\end{array}$ & Low risk & $\begin{array}{l}\text { All outcomes described were reported. Conclusions are in accordance with the } \\
\text { results }\end{array}$ \\
\hline Other bias & Low risk & None \\
\hline
\end{tabular}

Li 2003

Title: comparison of clinical efficacy and safety of 3 professional at-home tooth whitening systems
Trial design: randomised, 3-cell, parallel-group, investigator-blinded trial
Location: university
Language: English
Number of centres: 1
Recruitment period: not reported
Funding source: Discuss Dental

Participants

Participants: 23 to 67 years old

Total number: 90

Inclusion criteria:

- all subjects had a minimum 20 natural teeth, including at least 4 maxillary incisors with a shade at or darker A3

Exclusion criteria: not reported

Number randomised: not reported

Method of randomisation: randomisation scheme generated by a computer

Method of allocation concealment: reported

Method of blinding: reported

Number evaluated: 82

Total number of intervention groups: 3 groups

6.5\% hydrogen peroxide: 21 days, twice daily - 30-minute application

7.5\% hydrogen peroxide: 18 days, twice daily - 30-minute application

16\% carbamide peroxide: 21 days, overnight - 30-minute application

Duration of treatment: 18 to 21 days based on the intervention

Improvement in tooth shade: Vitapan shade guide: $\mathrm{B} 1$ to $\mathrm{C} 4$ (lightest to darkest shade ranking)
$\Delta \mathrm{L}, \mathrm{a}^{\star}, \mathrm{b}^{\star}$ and $\Delta \mathrm{W}$ using chromometre. Increase in $\mathrm{L}$ and $\mathrm{W}$ indicated whitening. Reduction in $\mathrm{b}^{\star}$ indicat-
ed whitening


Li 2003 (Continued)

Sample size calculation: not reported
Adverse effects: tooth sensitivity
Health-related quality of life: not reported
Key conclusions of the study authors: "All 3 systems were effective and safe. Nite White Excel (16\% CP)
resulted in significant greater shade reductions in periods between days 7,14, or 21 and baseline than
did the other 2 systems. Tooth sensitivity and gingival irritation was seen in all groups, but it was lower
in the Nite White Excel group"
Correspondence required: no

\section{Risk of bias}

\begin{tabular}{|c|c|c|}
\hline Bias & Authors' judgement & Support for judgement \\
\hline $\begin{array}{l}\text { Random sequence genera- } \\
\text { tion (selection bias) }\end{array}$ & Low risk & $\begin{array}{l}\text { Quote: "Qualified subjects were divided into ...... using randomisation scheme } \\
\text { generated by computer" }\end{array}$ \\
\hline $\begin{array}{l}\text { Allocation concealment } \\
\text { (selection bias) }\end{array}$ & Low risk & $\begin{array}{l}\text { Quote: "To ensure blinding, the group assignment and product distribution } \\
\text { was done by project coordinator who was not involved in the study" }\end{array}$ \\
\hline $\begin{array}{l}\text { Blinding of participants } \\
\text { and personnel (perfor- } \\
\text { mance bias) } \\
\text { All outcomes }\end{array}$ & Low risk & $\begin{array}{l}\text { Quote: "To ensure blinding, the group assignment and product distribution } \\
\text { was done by project coordinator who was not involved in the study" }\end{array}$ \\
\hline $\begin{array}{l}\text { Blinding of outcome as- } \\
\text { sessment (detection bias) } \\
\text { All outcomes }\end{array}$ & Low risk & $\begin{array}{l}\text { Quote: "To ensure blinding, the group assignment and product distribution } \\
\text { was done by project coordinator who was not involved in the study... while } \\
\text { clinical examiner performed examination without knowledge of treatment" }\end{array}$ \\
\hline $\begin{array}{l}\text { Incomplete outcome data } \\
\text { (attrition bias) } \\
\text { All outcomes }\end{array}$ & Low risk & $\begin{array}{l}\text { Quote: "A total of } 5 \text { subjects withdrew....3 did not show up.." } \\
\text { Comment: missing outcome data balanced in numbers across intervention } \\
\text { groups }\end{array}$ \\
\hline $\begin{array}{l}\text { Selective reporting (re- } \\
\text { porting bias) }\end{array}$ & Low risk & $\begin{array}{l}\text { All outcomes described were reported. Conclusions are in accordance with the } \\
\text { results }\end{array}$ \\
\hline Other bias & Low risk & None \\
\hline
\end{tabular}
mens

Trial design: stratified, double-blinded, parallel-group, randomised controlled trial

Location: Loma Linda University, USA

Language: English

Number of centres: 1

Recruitment period: not reported

Funding source: Colgate Pamolive 
Li 2004 (Continued)

Participants

Participants: 18 to 65 years old. Mean age 39.06 years

Total number: 120

Inclusion criteria:

- all maxillary and anterior teeth present

- teeth required to have a minimum A3 or darker shade on Vitapan

- pregnant, lactating

Exclusion criteria:

- with orthodontic appliances, crowns, tumours, periodontal disease

Number randomised: 120

Method of randomisation: not reported

Method of allocation concealment: not reported

Method of blinding: not reported

Number evaluated: 104.16 dropouts

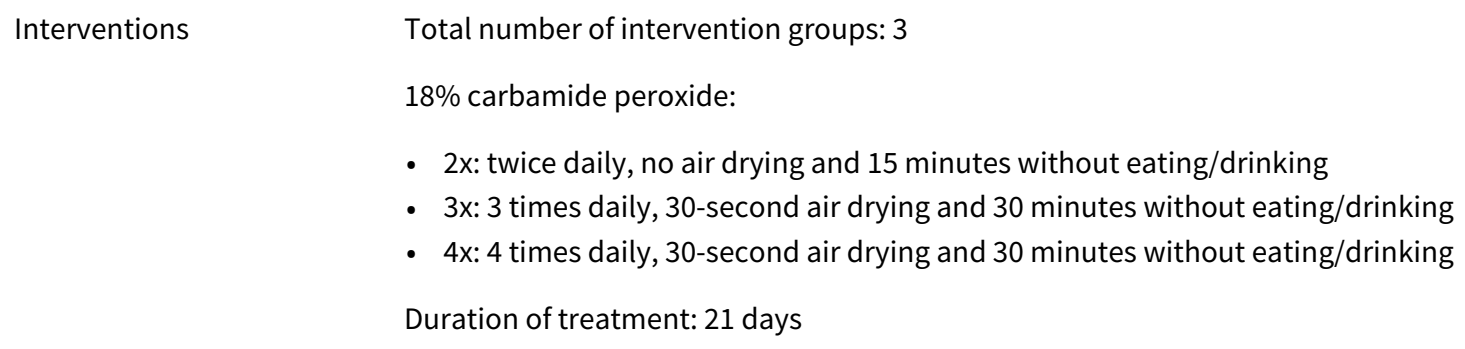

Outcomes

Improvement in tooth shade

Vita shade guide: lightest (C1) 1 to darkest (B4) 16

Oral tissue: normal/abnormal

Gingival Index: Loë and Silness Gingival Index: 0 absence of inflation, 1 mild, 2 moderate, 3 severe

Tooth sensitivity: 0 (no pain) to 10 (severe pain)

Opinion survey: 1 (more positive) to 5 (least positive)

Notes

Sample size calculation: not reported

Adverse effects: sensitivity

Health-related quality of life: reported

Key conclusions of the study authors: "Subjects who used $3 x$ and $4 x$ regimen achieved the greatest shade improvement. However those values were only 1 shade better than twice daily and no dry regimen. There was no significant difference between $3 x$ and $4 x$ regimens. Patients who used $2 x$ and $3 x$ regimens found it to be more convenient"

Correspondence required: no

Contact: DrYun Po Zhang; yun_zhzng@copal.com 
Li 2004 (Continued)

\section{Bias Authors' judgement Support for judgement}

Random sequence genera- Unclear risk Quote: "120 qualifying subjects.... randomly assigned within strata to 1 of the tion (selection bias) 3..." However, the method of randomisation is not mentioned

\begin{tabular}{|c|c|c|}
\hline $\begin{array}{l}\text { Allocation concealment } \\
\text { (selection bias) }\end{array}$ & Unclear risk & Not mentioned \\
\hline $\begin{array}{l}\text { Blinding of participants } \\
\text { and personnel (perfor- } \\
\text { mance bias) } \\
\text { All outcomes }\end{array}$ & Unclear risk & $\begin{array}{l}\text { Quote: "stratified, double-blinded, parallel-group, randomised controlled tri- } \\
\text { al." However, the method of blinding is not mentioned }\end{array}$ \\
\hline $\begin{array}{l}\text { Blinding of outcome as- } \\
\text { sessment (detection bias) } \\
\text { All outcomes }\end{array}$ & Unclear risk & $\begin{array}{l}\text { Quote: "stratified, double-blinded, parallel-group, randomised controlled tri- } \\
\text { al." However, the method of blinding is not mentioned }\end{array}$ \\
\hline $\begin{array}{l}\text { Incomplete outcome data } \\
\text { (attrition bias) } \\
\text { All outcomes }\end{array}$ & Low risk & $\begin{array}{l}\text { Quote: "104 subjects completed the clinical study: there were } 16 \text { dropouts.... } \\
\text { The data revealed the number was well balanced" } \\
\text { Comment: missing outcome data balanced in numbers across intervention } \\
\text { groups }\end{array}$ \\
\hline $\begin{array}{l}\text { Selective reporting (re- } \\
\text { porting bias) }\end{array}$ & Low risk & $\begin{array}{l}\text { All outcomes described were reported. Conclusions are in accordance with the } \\
\text { results }\end{array}$ \\
\hline Other bias & Low risk & None \\
\hline
\end{tabular}

Matis 1998

Methods

Title: efficacy and safety of a $10 \%$ carbamide peroxide bleaching gel

Trial design: double-blinded, randomised controlled trial

Location: Indiana University, School of Detitistry, Indianapolis, Indiana, USA

Language: English

Number of centres: 1

Recruitment period: not reported

Funding source: not reported

Participants

Participants: mean age 45.27 years

Total number: 60

Inclusion criteria:

- no soft or hard tissue pathosis (excluding small carious lesions and mild gingivitis)

- all 6 maxillary anterior teeth, with no more than $1 / 6$ of the facial surface restored

- non-smokers

Exclusion criteria:

- Loë and Silness Gingival Index (GT) > 1

Number randomised: 60 
Matis 1998 (Continued)

Method of randomisation: not reported

Method of allocation concealment: not reported

Method of blinding: dental assistant managed the grouping. All products were in identical syringes

Number evaluated: 59. 1 patient dropped out from 4th week from active group

Total number of intervention groups: 2 groups
$10 \%$ carbamide peroxide gel in tray
Placebo
Duration of treatment: 2 weeks

Change in tooth colour: Truebyte shade guide: 0 lightest, 25 darkest
Colourimetre: $b^{\star}$ : decreased $b^{\star}$ indicates reduced yellowness, $L$ : increased $L$ is increased brightness
Gingival sensitivity
Tooth sensitivity
Gastrointestinal sensitivity
Sensitivity scale: scale of 1 to $5: 1=$ no sensitivity, 2 = slight sensitivity, $3=$ moderate sensitivity, $4=$ con-
siderable sensitivity, $5=$ severe sensitivity
Sample size calculation: not reported
Adverse effects: gingival, tooth and gastrointestinal sensitivity was reported
Health-related quality of life: not reported
Key conclusions of the study authors: "The product used in this study is an effective and physiologi-
cally acceptable tooth whitening agent. Initial colour regression occurred within the first month for in-
cisors, and within 10 weeks for canines, but neither regressed back to baseline for the duration of this
6- month study"
Contact: Dr BA Matis; bmatis@iusd.iupui.edu

\section{Risk of bias}

Bias Authors' judgement Support for judgement

\begin{tabular}{lll}
$\begin{array}{l}\text { Random sequence genera- } \\
\text { tion (selection bias) }\end{array}$ & Unclear risk & $\begin{array}{l}\text { Quote: "60 patients were randomised into 2 equal subgroups balanced by age, } \\
\text { gender, and oral health status." However, method of randomisation is not } \\
\text { mentioned }\end{array}$ \\
\hline $\begin{array}{l}\text { Allocation concealment } \\
\text { (selection bias) }\end{array}$ & Unclear risk & Not mentioned \\
\hline
\end{tabular}

\begin{tabular}{|c|c|c|}
\hline $\begin{array}{l}\text { Blinding of participants } \\
\text { and personnel (perfor- } \\
\text { mance bias) } \\
\text { All outcomes }\end{array}$ & Low risk & $\begin{array}{l}\text { Quote: "Patients were given coded packages of Opalescence whitening gel. All } \\
\text { products were in identical syringes" }\end{array}$ \\
\hline $\begin{array}{l}\text { Blinding of outcome as- } \\
\text { sessment (detection bias) } \\
\text { All outcomes }\end{array}$ & Low risk & $\begin{array}{l}\text { Quote: "A dental assistant was responsible for group balancing, so that the } \\
\text { evaluators could continue to be blind to which treatment group each patient } \\
\text { was assigned" }\end{array}$ \\
\hline
\end{tabular}


Matis 1998 (Continued)

Incomplete outcome data Low risk At 2 weeks all 60 participants who were randomised were evaluated. However (attrition bias) 1 dropout was noticed after 4 weeks and 22 weeks reporting. Plausible effect size (difference in means) among missing outcomes not enough to have a clinically relevant impact on observed effect size

Selective reporting (re- Low risk
porting bias)

All outcomes described are reported adequately. Conclusions are in accordance with the results

Other bias Low risk None

\section{Matis 2000}

\section{Methods}

Title: clinical evaluation of bleaching agents of different concentrations

Trial design: split-mouth, randomised controlled trial

Location: Indiana University-Purdue University, Indianapolis, USA

Language: English

Number of centres: 1

Recruitment period: not reported

Funding source: Ultradent

\section{Participants}

\section{Participants: 26 to 73 years old. Mean age 50.4 years}

Total number: 25

Inclusion criteria:

- all 6 maxillary anterior teeth had to be present

- none of the maxillary anterior teeth have more than $1 / 6$ of its labial restoration

- at least 18 years old

- willing to refrain from the use of tobacco products during the study period

Exclusion criteria:

- use of professionally-applied or prescribed tooth whiteners, in-office bleaching, or mouthguard vital bleaching in the past 3 years

- incisors or canines lighter than B54 or darker than B85 on the Trubyte Bioforin Colour Ordered Shade Guide (Dentsply)

- gross pathosis in oral cavity (excluding caries)

- Loë and Silness Gingival Index score > 1

- pregnancy or lactation

- teeth discoloured by tetracycline staining

Number randomised: 25

Method of randomisation: not reported

Method of allocation concealment: not reported

Method of blinding: not reported

Number evaluated: 25 
Matis 2000 (Continued)

$10 \%$ carbamide peroxide

$15 \%$ carbamide peroxide with $0.11 \%$ fluoride ion

Duration of treatment: 14 days

\begin{tabular}{ll}
\hline Outcomes & Whitening of tooth. $\Delta \mathrm{L}, \mathrm{a}^{\star}, \mathrm{b}^{\star}$. Increase in $\Delta \mathrm{L}$ and reduction in $\mathrm{b}^{\star}$ indicated whitening \\
\hline Notes & Sample size calculation: not reported \\
& Adverse effects: tooth and gingival irritation \\
& Key conclusions of the study authors: "All 3 methods of evaluation revealed a significant difference in \\
the tooth lightness achieved by $10 \%$ and $15 \%$ products at 2 weeks but no significant difference at 6 \\
weeks. No statistically significant difference was found in gingival or tooth sensitivity" \\
Correspondence required: no \\
Contact: Dr Bruce A Matis; Bmatis@ijsd.njpui.edu
\end{tabular}

\section{Risk of bias}

\begin{tabular}{|c|c|c|}
\hline Bias & Authors' judgement & Support for judgement \\
\hline $\begin{array}{l}\text { Random sequence genera- } \\
\text { tion (selection bias) }\end{array}$ & Unclear risk & $\begin{array}{l}\text { Quote: "This was a double-blinded, 6-week study in which participants were } \\
\text { randomised into } 2 \text { groups by tooth shades." However, method is not men- } \\
\text { tioned }\end{array}$ \\
\hline $\begin{array}{l}\text { Allocation concealment } \\
\text { (selection bias) }\end{array}$ & Unclear risk & Not mentioned \\
\hline $\begin{array}{l}\text { Blinding of participants } \\
\text { and personnel (perfor- } \\
\text { mance bias) } \\
\text { All outcomes }\end{array}$ & Unclear risk & $\begin{array}{l}\text { Quote: "This was a double-blinded, } 6 \text {-week study in which participants were } \\
\text { randomised into } 2 \text { groups by tooth shades." However, method is not men- } \\
\text { tioned }\end{array}$ \\
\hline $\begin{array}{l}\text { Blinding of outcome as- } \\
\text { sessment (detection bias) } \\
\text { All outcomes }\end{array}$ & Unclear risk & $\begin{array}{l}\text { Quote: "This was a double-blinded, 6-week study in which participants were } \\
\text { randomised into } 2 \text { groups by tooth shades." However, method is not men- } \\
\text { tioned }\end{array}$ \\
\hline $\begin{array}{l}\text { Incomplete outcome data } \\
\text { (attrition bias) } \\
\text { All outcomes }\end{array}$ & Low risk & Quote: "All 25 subjects completed the study" \\
\hline $\begin{array}{l}\text { Selective reporting (re- } \\
\text { porting bias) }\end{array}$ & Low risk & $\begin{array}{l}\text { All outcomes described were reported. Conclusions are in accordance with the } \\
\text { results }\end{array}$ \\
\hline Other bias & Low risk & None \\
\hline
\end{tabular}

\section{Matis 2006}

Methods

Title: extended bleaching of tetracycline-stained teeth: a 5-year study

Trial design: split-mouth, randomised controlled trial

Location: Wuhan University School of Stomatology, China

Language: English 
Matis 2006 (Continued)

Number of centres: 1

Recruitment period: not reported

Funding source: Ultradent

Participants: not reported
Total number: 59
Inclusion criteria:
- 18 years of age
- willing to sign a consent form
- willing to return for periodic evaluations
- presence of 6 maxillary anterior teeth
- no more than $1 / 6$ of the facial surface of above teeth covered with restoration
- presence of tetracycline staining
Exclusion criteria:
- use of bleaching agents in past 3 years
- use of tobacco during previous 30 days
- study teeth lighter than A3
- history of disease that would interfere with study
- presence of gross pathology
Number randomised: 59
Method of randomisation: not reported
Method of allocation concealment: not reported
Method of blinding: not reported
Number evaluated: 44

Interventions

Total number of intervention groups: 3

$10 \%$ carbamide peroxide

$15 \%$ carbamide peroxide

$20 \%$ carbamide peroxide

Duration of treatment: 6 months

Outcomes Improvement in tooth colour: colourimeter readings in CIELa* $b^{\star}$ for Vitalescence

Restorative Masters Shade Guide

Sample size calculation: not reported
Adverse effects: tooth and gingival sensitivity
Key conclusions of the study authors: "The maximum lightening that occurred within 6 months hap-
pened during the first month of bleaching. Values increased the most during the bleaching of tetra-
cycline stained teeth. There were small changes in the green red or blue-yellow spectrums of colour
throughout the study. At 4.5 years post-bleaching, all 3 concentrations of bleaching agents had re-


Matis 2006 (Continued)

tained more than $65 \%$ of their original colour change. Increased tooth sensitivity occurs with higher concentrations of CP gels"

Correspondence required: no

Contact: Dr Bruce A Matis; Bmatis@ijsd.njpui.edu

\section{Risk of bias}

\begin{tabular}{|c|c|c|}
\hline Bias & Authors' judgement & Support for judgement \\
\hline $\begin{array}{l}\text { Random sequence genera- } \\
\text { tion (selection bias) }\end{array}$ & Unclear risk & $\begin{array}{l}\text { Quote: "At baseline evaluation, subjects were randomly assigned to } 1 \text { of } 6 \\
\text { groups." However, method is not mentioned }\end{array}$ \\
\hline $\begin{array}{l}\text { Allocation concealment } \\
\text { (selection bias) }\end{array}$ & Unclear risk & Not reported \\
\hline $\begin{array}{l}\text { Blinding of participants } \\
\text { and personnel (perfor- } \\
\text { mance bias) } \\
\text { All outcomes }\end{array}$ & Unclear risk & $\begin{array}{l}\text { Quote: "Subjects were not aware of the concentration of bleaching agent they } \\
\text { were using." However, method is not mentioned }\end{array}$ \\
\hline $\begin{array}{l}\text { Blinding of outcome as- } \\
\text { sessment (detection bias) } \\
\text { All outcomes }\end{array}$ & Unclear risk & Not reported \\
\hline $\begin{array}{l}\text { Incomplete outcome data } \\
\text { (attrition bias) } \\
\text { All outcomes }\end{array}$ & Low risk & $\begin{array}{l}\text { Of the } 59 \text { subjects who initially enrolled in the study, } 44 \text { completed the } 5 \text {-year } \\
\text { evaluation. Missing outcome data balanced in numbers across intervention } \\
\text { groups }\end{array}$ \\
\hline $\begin{array}{l}\text { Selective reporting (re- } \\
\text { porting bias) }\end{array}$ & Low risk & $\begin{array}{l}\text { All outcomes described were reported. Conclusions are in accordance with the } \\
\text { results }\end{array}$ \\
\hline Other bias & Low risk & None \\
\hline
\end{tabular}

\section{Mederios 2008}

Methods

Title: effectiveness of nightguard vital bleaching with $10 \%$ carbamide peroxide

Trial design: randomised controlled trial

Location: Universidade Federal do Rio Grande do Norte, Brazil

Language: English

Number of centres: 1

Recruitment period: not reported

Funding source: not reported

\section{Participants}

Participants: university students. 18 to 25 years old. Mean age 21.6 years

Total number: 50

Inclusion criteria:

- central and lateral upper incisors and had no fillings

- tooth sensitivity 
Mederios 2008 (Continued)

- endodontic treatment

- previous tooth bleaching

- periodontally healthy teeth

- non-smokers

Exclusion criteria: not reported

Number randomised: 50

Method of randomisation: raffle

Method of allocation concealment: not reported. Patients were randomly allotted to either group

Method of blinding: the placebo was placed in empty Opalescence PF packaging so that neither the volunteer nor the examiner knew which gel was being used

Number evaluated: 49

Total number of intervention groups: 2
$\begin{aligned} & \text { Interventions carbamide peroxide gel in tray } \\ & \text { Placebo } \\ & \text { Duration of treatment: } 21 \text { days }\end{aligned}$

Change in tooth shade: Vita shade guide - arranged from lightest to dark (1 light and 16 darkest)
Gingival Bleeding Index modified by Lang
Tooth sensitivity: yes or no
Patient satisfaction: satisfactory or non-satisfactory

Sample size calculation: not reported
Adverse effects: tooth sensitivity and gingival bleeding
Health-related quality of life: reported
Key conclusions of the study authors: "NGVB with 10\% carbamide peroxide, when use in the current
study, was effective for lightening tooth colour, both for the period immediately after treatment and for
the 6-month follow-up period. Of the 2 main side effects assessed, tooth sensitivity was more prevalent
than gingival irritation"
Contact: Dr Medeiros; cristinamedeiros@digizap.com.br

\section{Risk of bias}

\begin{tabular}{|c|c|c|}
\hline Bias & Authors' judgement & Support for judgement \\
\hline $\begin{array}{l}\text { Random sequence genera- } \\
\text { tion (selection bias) }\end{array}$ & Low risk & $\begin{array}{l}\text { Quote: "In a simple raffle, the } 50 \text { volunteers were randomly allocated to } 1 \text { of } \\
\text { the } 2 \text { groups.." }\end{array}$ \\
\hline $\begin{array}{l}\text { Allocation concealment } \\
\text { (selection bias) }\end{array}$ & Unclear risk & Not reported \\
\hline $\begin{array}{l}\text { Blinding of participants } \\
\text { and personnel (perfor- } \\
\text { mance bias) } \\
\text { All outcomes }\end{array}$ & Low risk & $\begin{array}{l}\text { Quote: "The placebo gel had the same physical characteristics as the exper- } \\
\text { imental gel. The placebo was placed in empty Opalescence PF packaging so } \\
\text { that neither the volunteer nor the examiner knew which gel was being used" }\end{array}$ \\
\hline
\end{tabular}


Mederios 2008 (Continued)

Blinding of outcome as- Unclear risk Quote: "All analysis were done by the evaluator" sessment (detection bias) All outcomes

Comment: but it is not mentioned whether the same evaluator dispensed the gel or not

Incomplete outcome data Low risk

(attrition bias)

Quote: "All volunteers completed the study.... The data of 1 volunteer from the

All outcomes placebo group were lost because of upper right lateral incisor anodontia"

Comment: plausible effect size (difference in means) among missing outcomes not enough to have a clinically relevant impact on observed effect size

$\begin{array}{ll}\begin{array}{l}\text { Selective reporting (re- } \\ \text { porting bias) }\end{array} & \begin{array}{l}\text { All outcomes described are reported. Conclusions are in accordance with the } \\ \text { results }\end{array}\end{array}$

porting bias)

Low risk None

Meireles 2010

Methods Title: double-blinded randomised clinical trial of 2 carbamide peroxide tooth bleaching agents: 2-year follow-up

Trial design: double-blinded, randomised clinical trial

Location: not reported

Language: English

Number of centres: 1

Recruitment period: not reported

Funding source: not reported

\section{Participants}

\section{Participants: not reported}

Total number: 183

Inclusion criteria:

- 6 anterior maxillary teeth with a colour shade $\mathrm{C} 1$ or darker

- evaluated teeth should not have more than $1 / 6$ of the buccal surface restored, and the restoration should not interfere with the spectrophotometer readings

- volunteers should have good oral health (no dental caries and periodontal disease)

- good general health (no disease that could interfere with the study results)

- volunteers should be at least 18 years old

Exclusion criteria:

- volunteers under orthodontic treatment or with tetracycline stained teeth

- volunteers reporting past or present hypersensitivity or those having non-vital anterior teeth

- volunteers that used tooth whiteners within the past 3 years

- smokers, pregnant or lactating women

- volunteers without schedule availability

Number randomised: 92

Method of randomisation: randomisation table

Method of allocation concealment: not reported 
Meireles 2010 (Continued)

Method of blinding: labels were removed

Number evaluated: 91

Interventions
Total number of intervention groups: 2
Control: carbamide peroxide $10 \%$ in tray
Experimental: carbamide peroxide $16 \%$ in tray
Duration of treatment: 2 hours per day for 3 weeks

\begin{tabular}{|c|c|}
\hline \multirow[t]{4}{*}{ Outcomes } & Improvement in tooth shade: $\Delta \mathrm{L}, \mathrm{a}^{\star}, \mathrm{b}^{\star}$ values recorded \\
\hline & Oral impact on daily performance (OIDP) \\
\hline & $\begin{array}{l}0=\text { no sensitivity; } 1=\text { mild sensitivity } 2=\text { moderate sensitivity } ; 3=\text { considerable sensitivity and } 4=\text { se }- \\
\text { vere sensitivity }\end{array}$ \\
\hline & $\begin{array}{l}\text { The self-reported general health was based on a Likert scale: excellent; very good; good; regular; bad } \\
\text { (latter categorized in excellent/very good and good/regular) }\end{array}$ \\
\hline
\end{tabular}

Notes
Adverse effects: tooth sensitivity
Health-related quality of life: reported
Key conclusions of the study authors: "The whitening effect evaluated by visual shade matching and
digital spectrophotometer remained similar after 6 months of bleaching treatment using any of the
carbamide peroxide concentrations tested. Additionally, the high consumption of staining beverage
and food had no influence in the whitening effect longevity. Quality of life is complex and encompasses
different domains. Although positive impact of the dental bleaching was detected, with patients show-
ing more their teeth without embarrassment, difficult in dental hygiene and pain resulting from the
treatment were also reported, and this can negatively impact daily performances. Dentists must con-
sider these aspects when performing aesthetics procedures"

This is a 2-year follow-up report of the previous study

Correspondence required: no

Contact: SS Meireles; soniasaeger@hotmail.com

\section{Risk of bias}

\begin{tabular}{|c|c|c|}
\hline Bias & Authors' judgement & Support for judgement \\
\hline $\begin{array}{l}\text { Random sequence genera- } \\
\text { tion (selection bias) }\end{array}$ & Low risk & Quote: "A randomisation table to allocate the participants in each study...." \\
\hline $\begin{array}{l}\text { Allocation concealment } \\
\text { (selection bias) }\end{array}$ & Unclear risk & Not mentioned \\
\hline $\begin{array}{l}\text { Blinding of participants } \\
\text { and personnel (perfor- } \\
\text { mance bias) } \\
\text { All outcomes }\end{array}$ & Low risk & $\begin{array}{l}\text { Quote: "The product concentration label was removed, therefore, the examin- } \\
\text { ers and participants were blinded to the agent" }\end{array}$ \\
\hline $\begin{array}{l}\text { Blinding of outcome as- } \\
\text { sessment (detection bias) } \\
\text { All outcomes }\end{array}$ & Unclear risk & Not mentioned \\
\hline
\end{tabular}


Meireles 2010 (Continued)

Incomplete outco (attrition bias)

Low risk

All outcomes
1 dropout. Plausible effect size (difference in means) among missing outcomes not enough to have a clinically relevant impact on observed effect size

\begin{tabular}{lll}
\hline $\begin{array}{l}\text { Selective reporting (re- } \\
\text { porting bias) }\end{array}$ & Low risk & $\begin{array}{l}\text { All outcomes described were reported. Conclusions are in accordance with the } \\
\text { results }\end{array}$ \\
\hline Other bias & Low risk & None
\end{tabular}

Mohan 2008

Methods
Participants

Title: clinical study to evaluate the efficacy of a novel tray-based tooth whitening system

Trial design: parallel, examiner-blinded, stratified, randomised controlled trial

Location: Department of Fixed and Removable Prosthodontics, Leeds Dental Institute, UK

Language: English

Number of centres: 1

Recruitment period: not reported

Funding source: not reported

Participants: 18 to 70 years old

Total number: 50

Inclusion criteria:

- available for the 2-week duration

- minimum of 20 uncrowned teeth with at least 6 upper front teeth without crowns or large restorations

- a minimum baseline shade of $A 3$ on 1 or more of the upper front teeth

Exclusion criteria:

- orthodontics bands, partial removable dentures

- advanced periodontal disease, tumours of the soft or hard tissues

- 5 or more carious lesions requiring immediate restorative treatment

- allergy history, participation in another clinical study within 1 month prior to the study, recent whitening or bleaching of teeth

- pregnant women

- medical conditions which would compromise the subject's safety

Number randomised: 50

Method of randomisation: not reported

Method of allocation concealment: not reported

Method of blinding: not reported

Number evaluated: 49.1 dropout from control group

Interventions
Total number of intervention groups: 2

Experimental: tray-based 6\% hydrogen peroxide

Control: no treatment 
Mohan 2008 (Continued)

Duration of treatment: 14 days

\begin{tabular}{l} 
Cutcomes \\
Oral irritation \\
Vita shade guide arranged based on lightness (B1 lightest and C4 darkest). 1 is lightest - 16 is darkest \\
$\mathrm{b}^{*}$ : decreased $\mathrm{b}^{*}$ indicates reduced yellowness; $\Delta \mathrm{L}$ : increased $\Delta \mathrm{L}$ is increased brightness \\
\hline Sample size calculation: not reported \\
Adverse effects: not reported \\
Health-related quality of life: not reported \\
Key conclusions of the study authors: "Significant tooth whitening was evident after 3 days treatment \\
with the tray-based whitening system and colour improved with continued usage over 14 days. It al- \\
so supports our previous study results that the WIO index is appropriate for assessing changes in tooth \\
whiteness" \\
Contact: Dr Naveen Mohan, Dental Health Unit, 3A Skelton House, Lloyd Street North, Manchester \\
Science Park, Manchester, England M15 \\
6SH, UK; iain.pretty@manchester.ac.uk (IA Pretty)
\end{tabular}

\section{Risk of bias}

Bias Authors' judgement Support for judgement

Random sequence genera- Unclear risk tion (selection bias)

Quote: "Balancing the 2 groups on the basis of baseline tooth colour, subjects were randomly assigned to either a tray-based bleaching system or a nontreatment control group." However, the method of randomisation is not mentioned

\begin{tabular}{|c|c|c|}
\hline $\begin{array}{l}\text { Allocation concealment } \\
\text { (selection bias) }\end{array}$ & Unclear risk & $\begin{array}{l}\text { Quote: "A parallel, examiner-blinded, stratified 2-group clinical study." No oth- } \\
\text { er details provided }\end{array}$ \\
\hline $\begin{array}{l}\text { Blinding of participants } \\
\text { and personnel (perfor- } \\
\text { mance bias) } \\
\text { All outcomes }\end{array}$ & Unclear risk & $\begin{array}{l}\text { Quote: "A parallel, examiner-blinded, stratified 2-group clinical study." No oth- } \\
\text { er details provided }\end{array}$ \\
\hline $\begin{array}{l}\text { Blinding of outcome as- } \\
\text { sessment (detection bias) } \\
\text { All outcomes }\end{array}$ & Unclear risk & $\begin{array}{l}\text { Quote: "A parallel, examiner-blinded, stratified 2-group clinical study." No oth- } \\
\text { er details provided }\end{array}$ \\
\hline $\begin{array}{l}\text { Incomplete outcome data } \\
\text { (attrition bias) } \\
\text { All outcomes }\end{array}$ & Low risk & $\begin{array}{l}\text { Quote: "1 dropout due to ill health was withdrawn from the study" } \\
\text { Comment: plausible effect size (difference in means ) among missing out- } \\
\text { comes not enough to have a clinically relevant impact on observed effect size }\end{array}$ \\
\hline $\begin{array}{l}\text { Selective reporting (re- } \\
\text { porting bias) }\end{array}$ & Low risk & $\begin{array}{l}\text { All outcomes described are reported. The conclusion is in accordance with re- } \\
\text { sults }\end{array}$ \\
\hline Other bias & Low risk & None \\
\hline
\end{tabular}


Mokhlis 2000

\section{Methods}

Title: clinical evaluation of carbamide peroxide and hydrogen peroxide whitening agents during daytime

Trial design: split-mouth, double-blinded, randomised controlled trial

Location: University Purdue, Indianapolis, USA

Language: English

Number of centres: 1

Recruitment period: not reported

Funding source: funded by Ultradent products Inc.
Participants: not reported

Total number: 24

Inclusion criteria:

- 6 maxillary anterior teeth present and free of any restorative material covering more than $1 / 6$ of their labial surfaces

- 6 anterior teeth darker than B54 and lighter than B85 on the Trubyte Bioform Colour Ordered Shade Guide (Dentsply Trubyte)

- at least 18 years of age

- willing to sign a consent form and able to return for periodic examinations

- willing to refrain from use of tobacco products during the study period

Exclusion criteria:

- medical condition that might interfere with the study results or require special attention

- Gingival Index score > 1 or gross pathology in the mouth

- tetracycline-stained teeth or having undergone endodontic therapy in any of the maxillary anterior teeth

- use of professionally-applied tooth whiteners within the past 5 years

- use of any kind of tobacco products during the past 30 days

- pregnant or lactating women

Number randomised: 24

Method of randomisation: reported

Method of allocation concealment: reported

Method of blinding: not reported

Number evaluated: 24

Interventions

Total number of intervention groups: 2

$20 \%$ carbamide peroxide gel

$7.5 \%$ hydrogen peroxide gel

Duration of treatment: 2 weeks
Change in colour: $\Delta \mathrm{L}, \mathrm{a}^{\star}, \mathrm{b}^{\star}$ values were recorded. Increase in $\mathrm{L}$ and reduction in $\mathrm{b}^{*}$ indicated whitening

Tooth and soft tissue sensitivity: 5-point scale: 1 none; 2 mild; 3 moderate; 4 considerable; and 5 severe 
Adverse effects: tooth and soft tissue sensitivity

Health-related quality of life: not reported

Key conclusions of the study authors: "Use of the $20 \%$ CP resulted in significantly more lightness than the $7.5 \%$ HP during the first 14 days of the study, but at the end of the study, there was no significant difference between products with regard to tooth lightness. In addition, the authors found no statistically significant difference between products with regard to gingival or tooth sensitivity"

Contact: GR Mokhlis, Indiana University School of Dentistry, Indianapolis, USA

\section{Risk of bias}

\begin{tabular}{|c|c|c|}
\hline Bias & Authors' judgement & Support for judgement \\
\hline $\begin{array}{l}\text { Random sequence genera- } \\
\text { tion (selection bias) }\end{array}$ & Low risk & $\begin{array}{l}\text { Quote: "The subjects were randomised according to the baseline shade guide } \\
\text { into } 2 \text { groups by a study monitor not directly involved in the study" }\end{array}$ \\
\hline $\begin{array}{l}\text { Allocation concealment } \\
\text { (selection bias) }\end{array}$ & Low risk & $\begin{array}{l}\text { Quote: "A study monitor assigned side of the mouth and the other gel to the } \\
\text { opposite side. The monitor then labelled each box of bleaching gel according- } \\
\text { ly" }\end{array}$ \\
\hline $\begin{array}{l}\text { Blinding of participants } \\
\text { and personnel (perfor- } \\
\text { mance bias) } \\
\text { All outcomes }\end{array}$ & Unclear risk & Double-blinded study but method of blinding not reported \\
\hline $\begin{array}{l}\text { Blinding of outcome as- } \\
\text { sessment (detection bias) } \\
\text { All outcomes }\end{array}$ & Unclear risk & Double-blinded study but method of blinding not reported \\
\hline $\begin{array}{l}\text { Incomplete outcome data } \\
\text { (attrition bias) } \\
\text { All outcomes }\end{array}$ & Low risk & Quote: "All 24 patients completed the study" \\
\hline $\begin{array}{l}\text { Selective reporting (re- } \\
\text { porting bias) }\end{array}$ & Low risk & $\begin{array}{l}\text { All outcomes described were reported. Conclusions are in accordance with the } \\
\text { results }\end{array}$ \\
\hline Other bias & Low risk & None \\
\hline
\end{tabular}

\section{Myers 2003}

Methods

Title: clinical evaluation of a $3 \%$ hydrogen peroxide tooth whitening gel

Trial design: double-blinded, placebo-controlled, randomised trial

Location: School of Dentistry, Medical College of Georgia, Augusta, Georgia, USA

Language: English

Number of centres: 1

Recruitment period: not reported

Funding source: Applied Dental Sciences

Participants

Participants: age not reported

Total number: 65 
Myers 2003 (Continued)

Inclusion criteria:

- 21 years of age and above

- A3 or darker on vita shade guide

Exclusion criteria:

- persons with significant medical problems or needing antibiotic premedication

- pregnant or nursing women

- persons with active dental caries or periodontal disease

- persons with a history of vital bleaching

- tetracycline staining

Number randomised: 65

Method of randomisation: randomisation table by statistician

Method of allocation concealment: syringes were labelled with the participant number. A single labelled syringe of gel was retained from each participant's box for later testing if needed

Method of blinding: not reported

Number evaluated: 65

Total number of intervention groups: 2
$3 \%$ hydrogen peroxide Nightguard gel
Placebo
Duration of treatment: 2 weeks

Outcomes Colour change: Vita shade guide arranged base on lightness: 1 lightest (C1) to 16 darkest (B4)

Dental sensitivity

Irritation to tongue, gingiva, and throat

Adverse effects: sensitivity in tongue and gingiva, dental sensitivity to hot and cold

Health-related quality of life: not reported

Key conclusions of the study authors: "Patient-applied NGVB with a 3\% hydrogen peroxide gel for 30 minutes 3 times a day for 2 weeks was effective in whitening teeth an average of 4.2 Vita shade tabs. The lightening effect was maintained at 6 months, and the side effects with this agent were similar to other whitening agents. The use of this material could be considered for patients who cannot comply with the regimen"

Contact: Michael L Myers, Department of Oral Rehabilitation, School of Dentistry, Medical College of Georgia, Augusta, Georgia 3091 USA; mmyers@mail.mcg.edu

\section{Risk of bias}

\begin{tabular}{lll}
\hline Bias & Authors' judgement & Support for judgement \\
\hline $\begin{array}{l}\text { Random sequence genera- } \\
\text { tion (selection bias) }\end{array}$ & Low risk & $\begin{array}{l}\text { Quote: "A randomisation table was prepared by the lead statistician (CMR), } \\
\text { who also maintained participant identification numbers used throughout the } \\
\text { study" }\end{array}$ \\
\hline
\end{tabular}


Myers 2003 (Continued)

Allocation concealment Low risk Quote: "A randomisation table was prepared by the lead statistician (CMR), (selection bias) who also maintained participant identification numbers used throughout the study.... The outside of the box and the individual syringes were labelled with the paticipant number"

\begin{tabular}{|c|c|c|}
\hline $\begin{array}{l}\text { Blinding of participants } \\
\text { and personnel (perfor- } \\
\text { mance bias) } \\
\text { All outcomes }\end{array}$ & Unclear risk & Double-blinded study but method of blinding not reported \\
\hline $\begin{array}{l}\text { Blinding of outcome as- } \\
\text { sessment (detection bias) } \\
\text { All outcomes }\end{array}$ & Unclear risk & Double-blinded study but method of blinding not reported \\
\hline $\begin{array}{l}\text { Incomplete outcome data } \\
\text { (attrition bias) } \\
\text { All outcomes }\end{array}$ & Low risk & No dropouts reported \\
\hline $\begin{array}{l}\text { Selective reporting (re- } \\
\text { porting bias) }\end{array}$ & Low risk & $\begin{array}{l}\text { All outcomes described were reported. Conclusions are in accordance with the } \\
\text { results }\end{array}$ \\
\hline Other bias & Low risk & None \\
\hline
\end{tabular}

\section{Nathoo 1994}

\section{Methods}

Title: clinical evaluation of Colgate Platinum professional tooth whitening system and Rembrandt lightening bleaching gel

Trial design: single-blinded, randomised, parallel-group clinical trial

Location: Colgate Palmolive Research Centre, USA

Language: English

Number of centres: 1

Recruitment period: not reported

Funding source: Colgate Palmolive

Participants

Participants: not reported

Total number: 40

Inclusion criteria: not mentioned

Exclusion criteria: not mentioned

Number randomised: 40 ( $n=20$ per group)

Method of randomisation: not reported

Method of allocation concealment: reported

Method of blinding: not reported

Number evaluated: 38 ( 1 dropout from each group: $n=19$ in each group) 
Nathoo 1994 (Continued)

10\% urea peroxide, 30 minutes twice daily (Colgate Platinum)

$10 \%$ urea peroxide, 30 minutes twice daily (Rembrandt lightening)

Duration of treatment: 2 weeks

\begin{tabular}{ll}
\hline Outcomes & $\begin{array}{l}\text { Improvement in tooth shade (objective assessment): increase in L and reduction in } b^{\star} \text { indicates whiten- } \\
\text { ing }\end{array}$ \\
Improvement in tooth shade (subjective assessment): Vita shade guide arrange in order of lightness: \\
percentage increase
\end{tabular}

\section{Risk of bias}

\begin{tabular}{|c|c|c|}
\hline Bias & Authors' judgement & Support for judgement \\
\hline $\begin{array}{l}\text { Random sequence genera- } \\
\text { tion (selection bias) }\end{array}$ & Unclear risk & $\begin{array}{l}\text { Quote: "Single-blinded, randomised, parallel-group clinical trial." However, } \\
\text { method of randomisation not reported }\end{array}$ \\
\hline $\begin{array}{l}\text { Allocation concealment } \\
\text { (selection bias) }\end{array}$ & Low risk & $\begin{array}{l}\text { Quote: "The identity of the products was concealed neither the subjects nor in- } \\
\text { vestigator were informed about the identity of products or to which group the } \\
\text { individual belonged" }\end{array}$ \\
\hline $\begin{array}{l}\text { Blinding of participants } \\
\text { and personnel (perfor- } \\
\text { mance bias) } \\
\text { All outcomes }\end{array}$ & Unclear risk & $\begin{array}{l}\text { Quote: "The identity of the products was concealed neither the subjects nor } \\
\text { investigator were informed about the identity of products nor to which group } \\
\text { the individual belonged" but details of the blinding method are not reported }\end{array}$ \\
\hline $\begin{array}{l}\text { Blinding of outcome as- } \\
\text { sessment (detection bias) } \\
\text { All outcomes }\end{array}$ & Unclear risk & $\begin{array}{l}\text { Quote: "Single-blinded, randomised, parallel-group clinical trial." However, } \\
\text { method is not reported }\end{array}$ \\
\hline \multirow{2}{*}{$\begin{array}{l}\text { Incomplete outcome data } \\
\text { (attrition bias) } \\
\text { All outcomes }\end{array}$} & Low risk & $\begin{array}{l}\text { Quote: "Of the } 40 \text { participants } 38 \text { participants completed the study ( } 1 \text { dropout } \\
\text { from each group)" }\end{array}$ \\
\hline & & $\begin{array}{l}\text { Comment: plausible effect size (difference in means) among missing outcomes } \\
\text { not enough to have a clinically relevant impact on observed effect size }\end{array}$ \\
\hline $\begin{array}{l}\text { Selective reporting (re- } \\
\text { porting bias) }\end{array}$ & Low risk & $\begin{array}{l}\text { All outcomes described were reported. Conclusions are in accordance with the } \\
\text { results }\end{array}$ \\
\hline Other bias & Low risk & None \\
\hline
\end{tabular}




\begin{tabular}{|c|c|}
\hline \multirow[t]{7}{*}{ Methods } & Title: comparative 7-day clinical evaluation of 2 tooth whitening products \\
\hline & Trial design: double-blinded, parallel-group, randomised controlled clinical trial \\
\hline & Location: not reported \\
\hline & Language: English \\
\hline & Number of centres: 1 \\
\hline & Recruitment period: not reported \\
\hline & Funding source: Colgate Palmolive \\
\hline \multirow[t]{9}{*}{ Participants } & Participants: 18 to 65 years old \\
\hline & Total number: 60 \\
\hline & Inclusion criteria: adults with shade darker than A3 \\
\hline & Exclusion criteria: not reported \\
\hline & Number randomised: 60 \\
\hline & Method of randomisation: not reported \\
\hline & Method of allocation concealment: not reported \\
\hline & Method of blinding: syringes were coded and wrapped \\
\hline & Number evaluated: 58 \\
\hline \multirow[t]{4}{*}{ Interventions } & Total number of intervention groups: 2 \\
\hline & $5 \%$ carbamide peroxide in tray \\
\hline & $10 \%$ carbamide peroxide in tray \\
\hline & Duration of treatment: 1 week, 6 to 8 hours per day \\
\hline \multirow[t]{2}{*}{ Outcomes } & Improvement in tooth shade: Vita shade guide: tabs arranged from dark to light \\
\hline & $\Delta \mathrm{L}, \mathrm{a}^{\star}, \mathrm{b}^{\star}$ values: increase in $\Delta \mathrm{L}$ and decrease in $\mathrm{b}^{*}$ indicates lightening of teeth \\
\hline \multirow[t]{6}{*}{ Notes } & Sample size calculation: not reported \\
\hline & Adverse effects: hypersensitivity: sensitivity as reported - as yes or no questions \\
\hline & Health-related quality of life: not reported \\
\hline & $\begin{array}{l}\text { Key conclusions of the study authors: "... whitening data showed that there was no significant differ- } \\
\text { ence between the } 2 \text { products after } 1 \text { week. The data suggest that these products are clinically equiva- } \\
\text { lent for tooth whitening. However, the subjective data collected on tooth hypersensitivity showed that } \\
\text { the product containing } 5 \% \text { carbamide peroxide was associated with less discomfort. Of the group using } \\
\text { the } 5 \% \text { carbamide peroxide product, } 20 \% \text { reported transient sensitivity of their teeth after product use } \\
\text { for } 1 \text { week compared with } 53 \% \text { of the group using the product with } 10 \% \text { carbamide peroxide. The prod- } \\
\text { uct containing } 5 \% \text { carbamide peroxide was associated with less tooth hypersensitivity after } 1 \text { week of } \\
\text { application" }\end{array}$ \\
\hline & Correspondence required: no \\
\hline & Contact: Saleem A Nathoo, Colgate Palmolive Research Centre, Piscataway, New Jersey, USA \\
\hline
\end{tabular}


Nathoo 2001 (Continued)

Risk of bias

\begin{tabular}{|c|c|c|}
\hline Bias & Authors' judgement & Support for judgement \\
\hline $\begin{array}{l}\text { Random sequence genera- } \\
\text { tion (selection bias) }\end{array}$ & Unclear risk & $\begin{array}{l}\text { Quote: "Double-blinded, randomised, controlled, parallel-group clinica trial." } \\
\text { However, method of randomisation is not mentioned }\end{array}$ \\
\hline $\begin{array}{l}\text { Allocation concealment } \\
\text { (selection bias) }\end{array}$ & Unclear risk & Not mentioned \\
\hline $\begin{array}{l}\text { Blinding of participants } \\
\text { and personnel (perfor- } \\
\text { mance bias) } \\
\text { All outcomes }\end{array}$ & Low risk & $\begin{array}{l}\text { Quote: "The identity of the products were wrapped, neither the investigator no } \\
\text { subjects were informed about.." }\end{array}$ \\
\hline $\begin{array}{l}\text { Blinding of outcome as- } \\
\text { sessment (detection bias) } \\
\text { All outcomes }\end{array}$ & Low risk & $\begin{array}{l}\text { Quote: "The identity of the products were wrapped, neither the investigator no } \\
\text { subjects were informed about.." }\end{array}$ \\
\hline \multirow{2}{*}{$\begin{array}{l}\text { Incomplete outcome data } \\
\text { (attrition bias) } \\
\text { All outcomes }\end{array}$} & Low risk & $\begin{array}{l}\text { Quote: "of the } 60 \text { participants who began the study } 29 \text { matched pairs }(n=58) \\
\text { remained throughout the study" }\end{array}$ \\
\hline & & $\begin{array}{l}\text { Comment: plausible effect size (difference in means) among missing out- } \\
\text { comes not enough to have a clinically relevant impact on observed effect size }\end{array}$ \\
\hline $\begin{array}{l}\text { Selective reporting (re- } \\
\text { porting bias) }\end{array}$ & Low risk & $\begin{array}{l}\text { All outcomes described were reported. Conclusions are in accordance with the } \\
\text { results }\end{array}$ \\
\hline Other bias & Low risk & None \\
\hline
\end{tabular}

\section{Nathoo 2002}

Methods

Title: efficacy of novel, non-tray paint on $18 \%$ carbamide peroxide gel

Trial design: double-blinded, parallel-group, randomised trial

Location: Colgate Palmolive Research Centre, USA

Language: English

Number of centres: 1

Recruitment period: not reported

Funding source: Colgate Palmolive

Participants

Participants: 18 to 58 years old. Mean age 39.79 years

Total number: 80

Inclusion criteria:

- A3 shade or darker

Exclusion criteria:

- orthodontic treatment

- restoration or crowns 
Nathoo 2002 (Continued)

Number randomised: not reported

Method of randomisation: method not reported

Method of allocation concealment: method not reported

Method of blinding: non-removable white packing with patient identification number

Number evaluated: 77

\begin{tabular}{ll}
\hline Interventions & Total number of intervention groups: 2 \\
$18 \%$ carbamide peroxide paint-on gel \\
Placebo \\
Duration of treatment: 21 days \\
\hline Change in tooth shade \\
Vita shade guide arranged base on lightness: 1 lightest (C1) to 16 darkest (B4) \\
\hline Sample size calculation: not reported \\
Adverse effects: not reported \\
Health-related quality of life: not reported \\
Key conclusions of the study authors: "Study showed that subjects' teeth in the liquid whitening gel- \\
treated group exhibited an overall improvement and a 3.5-shade difference compared with teeth in the \\
placebo gel group. No soft tissue adverse reaction were reported" \\
Contact: Saleem A Nathoo, Colgate Palmolive Research Centre, Piscataway, New Jersey, USA
\end{tabular}

\section{Risk of bias}

Bias Authors' judgement Support for judgement

Random sequence genera- Unclear risk Quote: "Qualifying subjects were then stratified... and randomly assigned tion (selection bias) within strata to one of the study treatment groups.." However, the method by which randomisation was done is not mentioned in the article

\begin{tabular}{lll}
\hline $\begin{array}{l}\text { Allocation concealment } \\
\text { (selection bias) }\end{array}$ & Unclear risk & Not mentioned \\
\hline $\begin{array}{l}\text { Blinding of participants } \\
\text { and personnel (perfor- } \\
\text { mance bias) }\end{array}$ & Low risk & $\begin{array}{l}\text { Quote: "For blinding, all products were overwrapped with a non-removable } \\
\text { white label containing a unique subject identification number" }\end{array}$ \\
$\begin{array}{l}\text { All outcomes } \\
\end{array}$ &
\end{tabular}

\begin{tabular}{lll}
\hline $\begin{array}{l}\text { Blinding of outcome as- } \\
\text { sessment (detection bias) } \\
\text { All outcomes }\end{array}$ & Unclear risk & Not mentioned \\
\hline $\begin{array}{l}\text { Incomplete outcome data } \\
\text { (attrition bias) }\end{array}$ & Low risk & Number of patients randomised were 80. However, there were 3 dropouts. \\
All outcomes & $\begin{array}{l}\text { Quote: "Subjects who did not complete the study dropped out for reasons un- } \\
\text { related to the use of the treatments or adverse events" }\end{array}$ \\
& $\begin{array}{l}\text { Comment: plausible effect size (difference in means) among missing outcomes } \\
\text { not enough to have a clinically relevant impact on observed effect size }\end{array}$
\end{tabular}


Nathoo 2002 (Continued)

$\begin{array}{ll}\begin{array}{l}\text { Selective reporting (re- } \\ \text { porting bias) }\end{array} & \text { Low risk } \\ \end{array}$

Other bias Low risk None

Nathoo 2003

Methods
Title: comparative clinical investigation of tooth whitening efficacy of 2 whitening gels

Trial design: randomised, double-blinded, parallel-group trial

Location: Oral Health Clinical Services, Piscataway, New Jersey, USA

Language: English

Number of centres: 1

Recruitment period: not reported

Funding source: Colgate Palmolive

Participants

Total number: 59

Inclusion criteria:

- 18 to 70 years of age

- all maxillary anterior present with no restoration

- no allergy to any of the ingredients of bleaching agent

- Vita shade of A3 or darker

Exclusion criteria:

- undergoing orthodontic treatment

- anterior tooth with prosthesis or veneers, crowns

- tumour of hard or soft tissues

- pregnant/lactating females

- oral prophylaxis in month or use of any whitening products

- any illness

Number randomised: 59

Method of randomisation: not mentioned

Method of allocation concealment: not mentioned

Method of blinding: not mentioned

Number evaluated: 59

Total number of intervention groups: 2

Gel I: $25 \%$ carbamide peroxide

Gel II: 8.7\% hydrogen peroxide

Duration of treatment: 3 weeks 


Sample size calculation: not reported
Adverse effects: none reported
Health-related quality of life: not reported
Key conclusions of the study authors: "The authors concluded that all subjects exhibited statically sig-
nificant tooth shade lightening. There was no significant difference in the between both gels for one
time night usage for $2 / 3$ weeks application. No adverse reaction was noted"
Correspondence required: no
Contact: Dr William DeVizio, William_devizio@colpal.com

\section{Risk of bias}

\begin{tabular}{|c|c|c|}
\hline Bias & Authors' judgement & Support for judgement \\
\hline $\begin{array}{l}\text { Random sequence genera- } \\
\text { tion (selection bias) }\end{array}$ & Unclear risk & $\begin{array}{l}\text { Quote: "double-blinded, randomised, controlled, parallel-group clinical trial." } \\
\text { However, method of randomisation is not reported }\end{array}$ \\
\hline $\begin{array}{l}\text { Allocation concealment } \\
\text { (selection bias) }\end{array}$ & Unclear risk & Not mentioned \\
\hline $\begin{array}{l}\text { Blinding of participants } \\
\text { and personnel (perfor- } \\
\text { mance bias) } \\
\text { All outcomes }\end{array}$ & Unclear risk & $\begin{array}{l}\text { Quote: "double-blinded, randomised, controlled, parallel-group clinical trial." } \\
\text { However, method of blinding is not reported }\end{array}$ \\
\hline $\begin{array}{l}\text { Blinding of outcome as- } \\
\text { sessment (detection bias) } \\
\text { All outcomes }\end{array}$ & Unclear risk & $\begin{array}{l}\text { Quote: "double-blinded, randomised, controlled, parallel-group clinical trial." } \\
\text { However, method of blinding is not reported }\end{array}$ \\
\hline $\begin{array}{l}\text { Incomplete outcome data } \\
\text { (attrition bias) } \\
\text { All outcomes }\end{array}$ & Low risk & Quote: "All the participants completed the study" \\
\hline $\begin{array}{l}\text { Selective reporting (re- } \\
\text { porting bias) }\end{array}$ & Low risk & $\begin{array}{l}\text { All outcomes described were reported. Conclusions are in accordance with the } \\
\text { results }\end{array}$ \\
\hline Other bias & Low risk & None \\
\hline
\end{tabular}

\section{Navarra 2014}

\section{Methods}

Title: effects of $210 \%$ carbamide peroxide Nightguard bleaching agents, with and without desensitizer, on enamel and sensitivity: an in vivo study

Trial design: blinded, randomised controlled trial

Location: not reported

Language: English

Number of centres: 1

Recruitment period: not reported 
Navarra 2014 (Continued)

Funding source: supported, in part, by grants from MIUR (Italy)
Participants: 20 to 50 years old. Mean age 25.3 years

Total number: 80

Inclusion criteria: not reported

Exclusion criteria:

- smoking, pregnancy or breastfeeding

- history of previous bleaching treatment

- dentine hypersensitivity caused by caries lesions

- fracture of restorations, chipped teeth, marginal gaps, post-operative sensitivity

- teeth with cervical fillings, and recent use of desensitizing toothpaste

Number randomised: 20

Method of randomisation: not reported

Method of allocation concealment: reported

Method of blinding: not reported

Number evaluated: 20
Total number of intervention groups: 2

$10 \%$ carbamide peroxide with fluoride and potassium nitrate in tray

$10 \%$ carbamide peroxide without desensitizing agents in tray

Duration of treatment: 2 weeks

Outcomes Improvement in tooth colour

$\Delta \mathrm{L}, \mathrm{a}^{\star}, \mathrm{b}^{\star}, \Delta \mathrm{W}$ values were recorded. Increase in $\Delta \mathrm{L}$ and reduction in $\mathrm{b}^{\star}$ indicated whitening

Sample size calculation: not reported
Adverse effects: sensitivity
Key conclusions of the study authors: "The use of $10 \%$ carbamide peroxide gel with fluoride and potas-
sium nitrate reduced the incidence of sensitivity during the bleaching treatment compared to a bleach-
ing agent that did not contain desensitizing agents. The bleaching effectiveness of the tested products
was comparable"
Correspondence required: no
Contact: M Cadenaro, m.cadenaro@fmc.units.it

\section{Risk of bias}

Bias Authors' judgement Support for judgement

\begin{tabular}{lll}
\hline $\begin{array}{l}\text { Random sequence genera- } \\
\text { tion (selection bias) }\end{array}$ & Unclear risk & $\begin{array}{l}\text { Quote: "An operator not involved in the research protocol performed the ran- } \\
\text { domisation." However, method of randomisation is not reported }\end{array}$ \\
\hline $\begin{array}{l}\text { Allocation concealment } \\
\text { (selection bias) }\end{array}$ & Low risk & $\begin{array}{l}\text { Quote: "allocated groups were recorded on cards contained in sequentially } \\
\text { numbered, sealed envelopes that were blindly assigned" }\end{array}$ \\
\hline
\end{tabular}


Navarra 2014 (Continued)

Blinding of participants and personnel (perfor-

Unclear risk mance bias)

All outcomes
Quote: "blinded randomised controlled trial." However, method of blinding is not reported

\begin{tabular}{|c|c|c|}
\hline $\begin{array}{l}\text { Blinding of outcome as- } \\
\text { sessment (detection bias) } \\
\text { All outcomes }\end{array}$ & Unclear risk & $\begin{array}{l}\text { Quote: "blinded randomised controlled trial." However, method of blinding is } \\
\text { not reported }\end{array}$ \\
\hline
\end{tabular}

Quote: "blinded randomised controlled trial." However, method of blinding is

No dropouts
Incomplete outcome data Low risk

(attrition bias)

All outcomes

\begin{tabular}{lll}
\hline $\begin{array}{l}\text { Selective reporting (re- } \\
\text { porting bias) }\end{array}$ & Low risk & $\begin{array}{l}\text { All outcomes described were reported. Conclusions are in accordance with the } \\
\text { results }\end{array}$ \\
\hline Other bias & Low risk & None \\
\hline
\end{tabular}

Oliveira 2013

Title: safety and efficacy of a high-adhesion whitening strip under extended wear regimen
Trial design: randomised, blinded, parallel-group trial
Location: not reported
Language: English
Number of centres: 1
Recruitment period: not reported
Funding source: Procter \& Gamble

Participants

Participants: 19 to 64 years old. Mean age 42 years

Total number: 29

Inclusion criteria:

- 18 to 65 years of age

- agree not to participate in any other oral/dental product studies during the course of this study

- delay of any elective dentistry (including dental prophylaxis) until the study had been completed

- refrain from the use of any non-study oral care products once assigned to treatment

- return for all scheduled visits and follow study procedures

- at least 4 gradable maxillary anterior teeth with a Vita shade score of A2 or darker

Exclusion criteria:

- teeth previously bleached using a professional bleaching treatment, over-the-counter bleaching kit, or investigational bleaching product

- undergoing treatment for gingivitis, periodontitis, or caries

- self-reported tooth sensitivity

- fixed orthodontic appliances on the facial surfaces of the maxillary arch

- dental crowns or large composite restorations on the facial surfaces of gradable maxillary anterior teeth

- oral pathosis requiring prompt treatment or gross neglect of oral home care, and/or other signs indicating that the integrity of the data collected for that subject was compromised 
Oliveira 2013 (Continued)

- teeth that could not be imaged

- meaningful malocclusion that would impact on treatment or imaging

- teeth with severe or atypical intrinsic staining, such as that caused by tetracycline, fluorosis or hypocalcification

Number randomised: 29

Method of randomisation: not reported

Method of allocation concealment: not reported

Method of blinding: blinded test kits

Number evaluated: 28

\begin{tabular}{ll}
\hline Interventions & Total number of intervention groups: 2 \\
& $9.5 \%$ hydrogen peroxide gel strips \\
$10 \%$ hydrogen peroxide gel strips & \\
Duration of treatment: 9 days
\end{tabular}

Outcomes Improvement in tooth shade.

$\Delta \mathrm{L}, \mathrm{a}^{\star}, \mathrm{b}^{\star}$ values: increase in $\Delta \mathrm{L}$ and decrease in $\mathrm{b}^{\star}$ indicates lightening of teeth

Sample size calculation: not reported
Adverse effects: tooth sensitivity and oral irritation
Health-related quality of life: not reported
Key conclusions of the study authors: "The 2-hour regimen for the $9.5 \%$ hydrogen peroxide high-adhe-
sion whitening strip was more efficient for tooth whitening than the 30 -minute regimen of $10 \%$ hydro-
gen peroxide whitening strip. Both treatments were well tolerated and the use of the test products dur-
ing the study time frame was considered safe"

Contact: GM Oliveira, gmoliv03@louisville.edu

\section{Risk of bias}

Bias Authors' judgement Support for judgement

Random sequence genera- Unclear risk Quote: "..subjects were randomly assigned to 1 of the 2 groups.... properly baltion (selection bias) anced for age and baseline tooth colour." However, method of randomisation is not reported

\begin{tabular}{lll}
\hline $\begin{array}{l}\text { Allocation concealment } \\
\text { (selection bias) }\end{array}$ & Unclear risk & Not reported \\
\hline $\begin{array}{l}\text { Blinding of participants } \\
\text { and personnel (perfor- } \\
\text { mance bias) } \\
\text { All outcomes }\end{array}$ & Unclear risk & $\begin{array}{l}\text { Quote: "A randomised, blinded, parallel group, single centre..... All test prod- } \\
\text { ucts and related instructions for use were packaged in blinded test kits with } \\
\text { appropriate research labeling for distribution." No other details given }\end{array}$ \\
\hline $\begin{array}{l}\text { Blinding of outcome as- } \\
\text { sessment (detection bias) } \\
\text { All outcomes }\end{array}$ & Unclear risk & $\begin{array}{l}\text { Quote: "A randomised, blinded, parallel group, single centre..... oral examina- } \\
\text { tion was conducted by an examiner blinded to treatment assignment to identi- } \\
\text { fy possible changes in oral status..." No other details given }\end{array}$ \\
\hline $\begin{array}{l}\text { Incomplete outcome data } \\
\text { (attrition bias) }\end{array}$ & Low risk & Quote: "1 subject in the 2-hour strip group missed the day 5 visit" \\
\hline
\end{tabular}


Oliveira 2013 (Continued)

All outcomes
Comment: plausible effect size (difference in means) among missing outcomes not enough to have a clinically relevant impact on observed effect size

$\begin{array}{ll}\begin{array}{l}\text { Selective reporting (re- } \\ \text { porting bias) }\end{array} & \text { Low risk } \\ \text { results }\end{array}$

Other bias Low risk None

Papas 2009

Methods

Title: placebo-controlled clinical trial of use of $10 \%$ hydrogen peroxide whitening strips for medication-induced xerostomia

Trial design: randomised, double-blinded, placebo-controlled clinical trial

Location: University School of Dental Medicine and New England Medical Center, USA

Language: English

Number of centres: 1

Recruitment period: not reported

Funding source: supported, in part, by Procter \& Gamble

\begin{tabular}{|c|c|}
\hline \multirow[t]{16}{*}{ Participants } & Participants: mean age 50 years \\
\hline & Total number: 42 \\
\hline & Inclusion criteria: \\
\hline & - 4 or more anterior teeth \\
\hline & $\begin{array}{l}\text { - xerogenic medication history and symptoms, subjects had to present with an unstimulated salivary } \\
\text { flow^ } 0.2 \mathrm{ml} / \mathrm{min}\end{array}$ \\
\hline & - Vita shade score of $\mathrm{A} 2$ or darker \\
\hline & - adults 18 and above \\
\hline & Exclusion criteria: \\
\hline & - previous vital bleaching, apparent caries, periodontal disease \\
\hline & - chlorhexidine mouthwas used \\
\hline & - history of dentine hypersensitivity \\
\hline & Number randomised: 42 \\
\hline & Method of randomisation: not reported \\
\hline & Method of allocation concealment: not reported \\
\hline & Method of blinding: identical package and coded \\
\hline & Number evaluated: 40 ( 1 withdrew and 1 did not report after 15 days) \\
\hline \multirow[t]{4}{*}{ Interventions } & Total number of intervention groups: 2 \\
\hline & Control: placebo \\
\hline & Experimental: $10 \%$ hydrogen peroxide whitening strips \\
\hline & Duration of treatment: 2 weeks \\
\hline
\end{tabular}


Papas 2009 (Continued)

\begin{tabular}{|c|c|}
\hline Outcomes & $\begin{array}{l}\text { Tooth colour change } \\
\text { Oral irritation and sensitivity } \\
\mathrm{b}^{\star} \text { : decreased } \mathrm{b}^{\star} \text { indicates reduced yellowness; } \Delta \mathrm{L} \text { : increased } \Delta \mathrm{L} \text { is increased brightness }\end{array}$ \\
\hline Notes & $\begin{array}{l}\text { Sample size calculation: not reported } \\
\text { Adverse effects: tooth sensitivity and gingival irritation } \\
\text { Key conclusions of the study authors: "At day 8, the peroxide group experienced colour improvement } \\
\text { relative to baseline and placebo. Mild and transient tooth sensitivity represented the most common ad- } \\
\text { verse events. No subject discontinued treatment due to a product-related adverse event" } \\
\text { Contact: Athena S Papas, Tufts School of Dental Medicine, } 1 \text { Kneeland Street, Boston, MA 02111, USA; } \\
\text { athena.papas@tufts.edu }\end{array}$ \\
\hline
\end{tabular}

\section{Risk of bias}

Bias Authors' judgement Support for judgement

Random sequence genera- Unclear risk tion (selection bias)

Quote: "A randomised double-blinded placebo-controlled clinical trial was conducted... eligible subjects were randomly assigned to .."

However, the method of randomisation is not mentioned

\begin{tabular}{lll}
\hline $\begin{array}{l}\text { Allocation concealment } \\
\text { (selection bias) }\end{array}$ & Unclear risk & Not mentioned \\
\hline $\begin{array}{l}\text { Blinding of participants } \\
\text { and personnel (perfor- } \\
\text { mance bias) }\end{array}$ & Low risk & $\begin{array}{l}\text { Quote: "Except for the presence or absence of peroxide, the test strips were } \\
\text { identical in appearance. The test strips (peroxide or placebo) were dispensed } \\
\text { in a small, white, cardboard box with instructions specifying twice daily strip } \\
\text { application for } 30 \text { minutes before toothbrushing. To further ensure blinding, } \\
\text { each box was labelled only with a unique subject identification number and } \\
\text { necessary contact information" }\end{array}$ \\
\hline
\end{tabular}

\begin{tabular}{lll}
\hline $\begin{array}{l}\text { Blinding of outcome as- } \\
\text { sessment (detection bias) } \\
\text { All outcomes }\end{array}$ & Unclear risk & Not reported \\
\hline $\begin{array}{l}\text { Incomplete outcome data } \\
\text { (attrition bias) }\end{array}$ & Low risk & Quote: "1 subject from placebo group voluntarily withdrew from the study.." \\
All outcomes & $\begin{array}{l}\text { Comment: } 1 \text { participant withdrew and } 1 \text { did not report after } 15 \text { days. Plausible } \\
\text { effect size (difference in means) among missing outcomes not enough to have } \\
\text { a clinically relevant impact on observed effect size }\end{array}$
\end{tabular}

\begin{tabular}{lll}
\hline $\begin{array}{l}\text { Selective reporting (re- } \\
\text { porting bias) }\end{array}$ & Low risk & $\begin{array}{l}\text { All outcomes mentioned are reported adequately. Conclusions are in accor- } \\
\text { dance with the results }\end{array}$ \\
\hline Other bias & Low risk & None \\
\hline
\end{tabular}

Porciani 2006

\begin{tabular}{ll}
\hline Methods & Title: whitening effect by stain inhibition from a chewing gum with sodium hexametaphosphate in a \\
controlled 12-week single-blinded trial
\end{tabular}

Trial design: randomised, single-blinded, cross-over trial 
Porciani 2006 (Continued)

Location: University of Siena, Dental School, Siena, Italy

Language: English

Number of centres: 1

Recruitment period: not reported

Funding source: Perfetti Van Melle

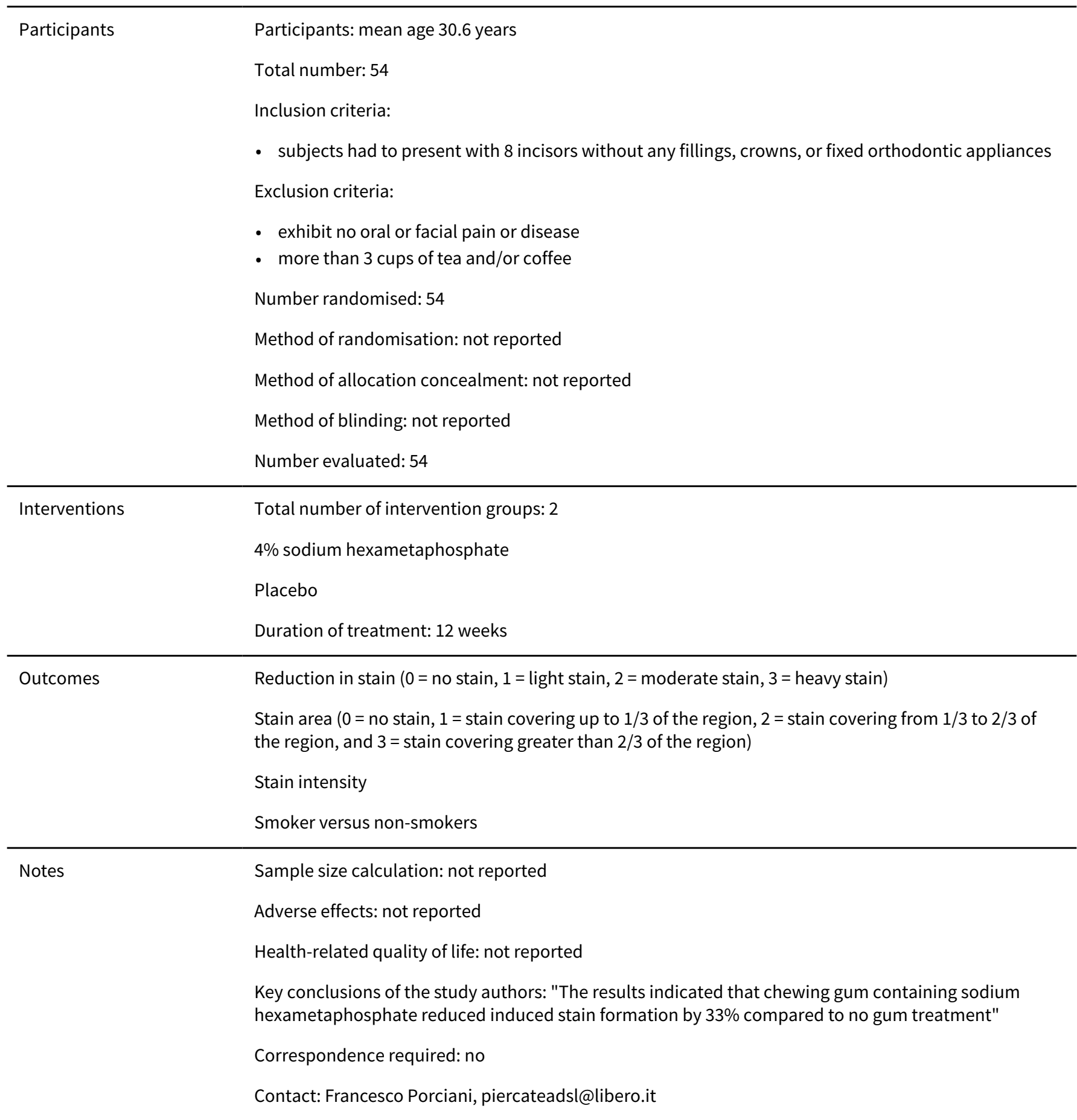

\section{Risk of bias}


Porciani 2006 (Continued)

\section{Bias Authors' judgement Support for judgement}

Random sequence genera- Unclear risk Quote: "were randomly assigned to the test gum or no-gum group.." However, tion (selection bias) method of randomisation is not reported

\begin{tabular}{|c|c|c|}
\hline $\begin{array}{l}\text { Allocation concealment } \\
\text { (selection bias) }\end{array}$ & Unclear risk & Not mentioned \\
\hline $\begin{array}{l}\text { Blinding of participants } \\
\text { and personnel (perfor- } \\
\text { mance bias) } \\
\text { All outcomes }\end{array}$ & Unclear risk & Not mentioned \\
\hline $\begin{array}{l}\text { Blinding of outcome as- } \\
\text { sessment (detection bias) } \\
\text { All outcomes }\end{array}$ & Low risk & $\begin{array}{l}\text { Quote: "the teeth were scored for stain deposits by the same examiner who } \\
\text { was blinded to the product assignments" }\end{array}$ \\
\hline $\begin{array}{l}\text { Incomplete outcome data } \\
\text { (attrition bias) } \\
\text { All outcomes }\end{array}$ & Low risk & $\begin{array}{l}\text { Quote: "All } 54 \text { subjects ( } 27 \text { females and } 27 \text { males) initially enrolled in the study } \\
\text { completed it" }\end{array}$ \\
\hline $\begin{array}{l}\text { Selective reporting (re- } \\
\text { porting bias) }\end{array}$ & Low risk & $\begin{array}{l}\text { All outcomes described were reported. Conclusions are in accordance with the } \\
\text { results }\end{array}$ \\
\hline Other bias & Low risk & None \\
\hline
\end{tabular}

Porciani 2010

Methods Title: effect on dental stain occurrence by chewing gum containing sodium tripolyphosphate

Trial design: double-blinded, parallel-group, randomised controlled trial

Location: not reported

Language: English

Number of centres: 1

Recruitment period: not reported

Funding source: Perfetti van Melle

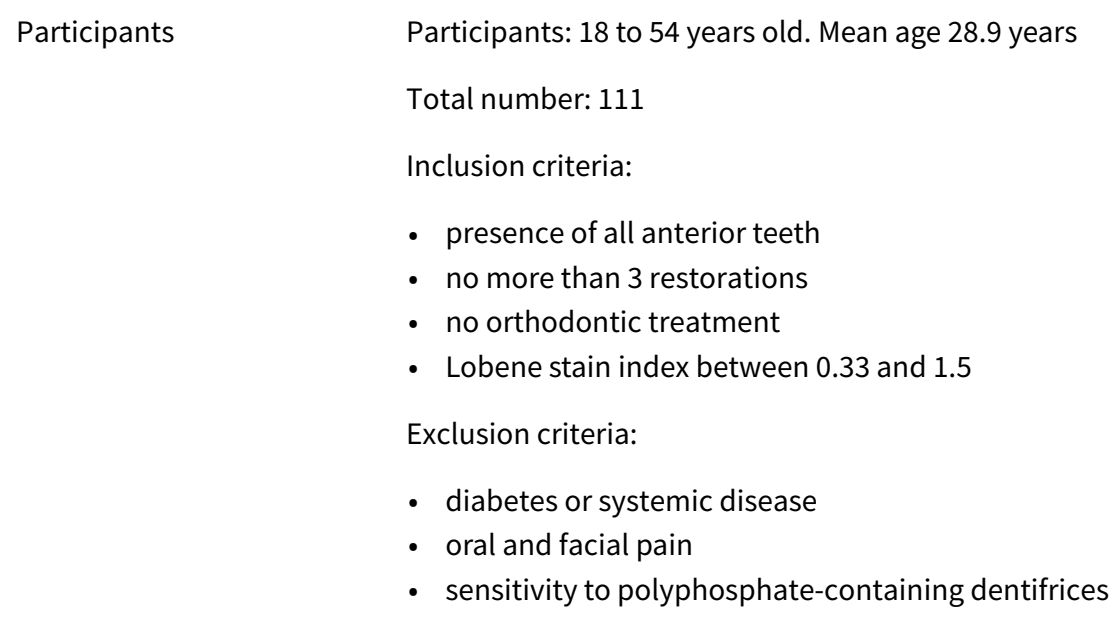


Porciani 2010 (Continued)

Number randomised: 111

Method of randomisation: random table

Method of allocation concealment: not reported

Method of blinding: similar looking package, shape, flavour and weight of the chewing gum. Participants instructed not to discuss the treatment they

were receiving

Number evaluated: 108.3 participants dropped out from experiment group

\begin{tabular}{ll}
\hline Interventions & Total number of intervention groups: 2 \\
& Experimental: sodium tripolyphosphate (1\%) containing gum \\
& Control: placebo \\
& Duration of treatment: 6 weeks \\
\hline Outcomes & Reduction in stain intensity and extent of stain \\
& Lobene modified index: smaller value shows improvement \\
& Stain composite index: smaller value shows improvement \\
\hline Notes & Sample size calculation: not reported \\
& Adverse effects: not reported \\
& Health-related quality of life: not reported \\
& Key conclusions of the study authors: "This trial showed a reduction in dental stain by a chewing gum \\
containing sodium tripolyphosphate after 6 weeks" \\
Contact: PF Porciani, piercateadsl@libero.it
\end{tabular}

\section{Risk of bias}

\begin{tabular}{|c|c|c|}
\hline Bias & Authors' judgement & Support for judgement \\
\hline $\begin{array}{l}\text { Random sequence genera- } \\
\text { tion (selection bias) }\end{array}$ & Low risk & $\begin{array}{l}\text { Quote: "Each subject entered in the test or control group using a random ta- } \\
\text { ble.." }\end{array}$ \\
\hline $\begin{array}{l}\text { Allocation concealment } \\
\text { (selection bias) }\end{array}$ & Unclear risk & Not mentioned \\
\hline $\begin{array}{l}\text { Blinding of participants } \\
\text { and personnel (perfor- } \\
\text { mance bias) }\end{array}$ & Low risk & $\begin{array}{l}\text { Quote: "Both chewing gums had the same flavour, weight, shape, colour, and } \\
\text { packaging so that the participants were blinded as to the identity of the gum" }\end{array}$ \\
\hline All outcomes & & $\begin{array}{l}\text { Comment: additionally, participants were instructed not to tell other subjects } \\
\text { to which group they were assigned in order to minimize inadvertent disclosure } \\
\text { to the study participants and staff }\end{array}$ \\
\hline
\end{tabular}

Blinding of outcome as- Low risk Quote: "Data were scored by the same blinded operator for all measurements" sessment (detection bias) All outcomes

\begin{tabular}{|c|c|c|}
\hline $\begin{array}{l}\text { Incomplete outcome data } \\
\text { (attrition bias) }\end{array}$ & Low risk & $\begin{array}{l}\text { Quote: "... } 3 \text { eventually dropped out, though the cause was unrelated to } \\
\text { polyphosphates" }\end{array}$ \\
\hline
\end{tabular}


Porciani 2010 (Continued)

Comment: plausible effect size (difference in means) among missing outcomes not enough to have a clinically relevant impact on observed effect size

\begin{tabular}{lll}
\hline $\begin{array}{l}\text { Selective reporting (re- } \\
\text { porting bias) }\end{array}$ & Low risk & $\begin{array}{l}\text { All outcomes mentioned were reported adequately. Conclusion reflected } \\
\text { study results (in the abstract) }\end{array}$ \\
\hline Other bias & Low risk & None \\
\hline
\end{tabular}

\section{Russell 1996}

\section{Methods}

Title: dentist-supervised home bleaching with $10 \%$ carbamide peroxide gel

Trial design: double-blinded, randomised controlled trial

Location: Medical College of Georgia, USA

Language: English

Number of centres: 1

Recruitment period: not reported

Funding source: not reported

\section{Participants}

Participants: 22 to 79 years. Mean age 43.9 years

Total number: 50

Inclusion criteria:

- healthy adults

Exclusion criteria:

- previous vital bleaching

- apparent caries, periodontal disease

- orthodontic appliances

- anterior restorations

- history of dentine hypersensitivity

- pregnant or nursing woman

- tetracycline stains

- on antibiotic medication

Number randomised: 50

Method of randomisation: randomisation table

Method of allocation concealment: not reported

Method of blinding: both gels were provided by the manufacturer in small kits containing syringes

Number evaluated: 50

Experimental: carbamide peroxide 10\% tray

Control: placebo

Duration of treatment: 2 weeks (follow-up of 6 months) 
Russell 1996 (Continued)

Outcomes
Lightening of teeth

Vita shade guide arrange in ascending order for lightness. Rank 1: darkest (B4), rank 16: lightness (C1)

\section{Notes}

Sample size calculation: not reported

Adverse effects: not reported

Health-related quality of life: not reported

Key conclusions of the study authors: "The $10 \%$ carbamide peroxide product and treatment regimen for vital bleaching used in this study have been shown to produce a significant lightening effect immediately after treatment consistent with other studies. In addition, this study shows that the lightening effect lasts at least 6 months for the majority of subjects"

Contact: Carl M Russell, Office of Biostatistics, Medical College of Georgia, Augusta, GA 30912-4900, USA

\section{Risk of bias}

\begin{tabular}{|c|c|c|}
\hline Bias & Authors' judgement & Support for judgement \\
\hline $\begin{array}{l}\text { Random sequence genera- } \\
\text { tion (selection bias) }\end{array}$ & Low risk & $\begin{array}{l}\text { Quote: "Randomisation table containing subject identification numbers and } \\
\text { group assignment was prepared by the lead statistician" }\end{array}$ \\
\hline $\begin{array}{l}\text { Allocation concealment } \\
\text { (selection bias) }\end{array}$ & Unclear risk & Not done \\
\hline $\begin{array}{l}\text { Blinding of participants } \\
\text { and personnel (perfor- } \\
\text { mance bias) } \\
\text { All outcomes }\end{array}$ & Low risk & $\begin{array}{l}\text { Quote: "The placebo treatment gel was the same as the active gel except the } \\
\text { carbamide peroxide was omitted. Both gels were provided by the manufactur- } \\
\text { er in small kits containing syringes.." }\end{array}$ \\
\hline $\begin{array}{l}\text { Blinding of outcome as- } \\
\text { sessment (detection bias) } \\
\text { All outcomes }\end{array}$ & Unclear risk & Not reported \\
\hline $\begin{array}{l}\text { Incomplete outcome data } \\
\text { (attrition bias) } \\
\text { All outcomes }\end{array}$ & Low risk & $\begin{array}{l}\text { No dropouts reported. The number randomised were the number assessed in } \\
\text { both groups over the } 6 \text {-month period }\end{array}$ \\
\hline $\begin{array}{l}\text { Selective reporting (re- } \\
\text { porting bias) }\end{array}$ & Low risk & $\begin{array}{l}\text { All outcomes described are reported adequately. Conclusions are in accor- } \\
\text { dance with the results }\end{array}$ \\
\hline Other bias & Low risk & None \\
\hline
\end{tabular}

Shahidi 2005

Methods Title: randomised controlled trial of $10 \%$ hydrogen peroxide whitening strips

Trial design: randomised, double-blinded clinical trial

Location: Hill Top Research Inc, USA

Language: English

Number of centres: 1

Recruitment period: not reported 
Shahidi 2005 (Continued)

Funding source: Procter \& Gamble

\begin{tabular}{|c|c|}
\hline Participants & Participants: 19 to 48 years old. Mean age 32.4 years \\
\hline & Total number: 40 \\
\hline & Inclusion criteria: not reported \\
\hline & Exclusion criteria: \\
\hline & - no previous tooth whitening \\
\hline & - sensitivity or exclusive restoration \\
\hline & Number randomised: 40 \\
\hline & Method of randomisation: not reported \\
\hline & Method of allocation concealment: not reported \\
\hline & Method of blinding: reported \\
\hline & Number evaluated: 35 \\
\hline
\end{tabular}

Interventions

Total number of intervention groups: 2

$6 \%$ hydrogen peroxide $0.2 \mathrm{~mm}$ strip

$10 \%$ hydrogen peroxide $0.13 \mathrm{~mm}$ strip

Duration of treatment: 2 weeks

Outcomes Improvement in tooth shade.

$\Delta \mathrm{L}, \mathrm{a}^{\star}, \mathrm{b}^{\star}$ values: increase in $\Delta \mathrm{L}$ and decrease in $\mathrm{b}^{\star}$ indicates lightening of teeth

Notes Sample size calculation: not reported

Adverse effects: tooth sensitivity and oral irritation

Key conclusions of the study authors: "Vital bleaching with $10 \%$ hydrogen peroxide strips at 1 week was as effective as $6 \%$ hydrogen peroxide strips used for 2 weeks. At the end of 2 weeks $10 \%$ hydrogen peroxide was better than $6 \%$ hydrogen peroxide. Both treatments were generally well tolerated, with mild and transient tooth sensitivity or oral irritation representing the most common adverse events"

Contact: Robert Geralch, geralchgw@pg.com

\section{Risk of bias}

\begin{tabular}{lll}
\hline Bias & Authors' judgement & Support for judgement \\
\hline $\begin{array}{l}\text { Random sequence genera- } \\
\text { tion (selection bias) }\end{array}$ & Unclear risk & $\begin{array}{l}\text { Quote: "Randomised, double-blinded clinical trial.... Subjects were random- } \\
\text { ly assigned to } 1 \text { of the strip groups." However, the method of randomisation is } \\
\text { not reported }\end{array}$ \\
\hline $\begin{array}{l}\text { Allocation concealment } \\
\text { (selection bias) }\end{array}$ & Unclear risk & Not mentioned \\
\hline $\begin{array}{l}\text { Blinding of participants } \\
\text { and personnel (perfor- } \\
\text { mance bias) } \\
\text { All outcomes }\end{array}$ & Low risk & $\begin{array}{l}\text { Quote: "The test and experimental strips were identical in size... each strip was } \\
\text { packaged in an individual white foil pouch, with subject identification num- } \\
\text { ber" }\end{array}$ \\
\hline
\end{tabular}


Shahidi 2005 (Continued)
Blinding of outcome as-
Unclear risk
Not mentioned sessment (detection bias)
All outcomes

\begin{tabular}{lll}
\hline $\begin{array}{l}\text { Incomplete outcome data } \\
\text { (attrition bias) } \\
\text { All outcomes }\end{array}$ & Unclear risk & $\begin{array}{l}5 \text { dropouts at the end of the trial. Reason for dropouts not mentioned. We are } \\
\text { not sure if the plausible effect size (difference in means) among missing out- } \\
\text { comes may have a clinically relevant impact on observed effect size }\end{array}$ \\
\hline $\begin{array}{l}\text { Selective reporting (re- } \\
\text { porting bias) }\end{array}$ & Low risk & $\begin{array}{l}\text { All outcome described are reported. Conclusions are in accordance with the } \\
\text { results }\end{array}$ \\
\hline Other bias & Low risk & None
\end{tabular}

\section{Swift 2004}

Methods

Title: 3-week clinical trial of a $14 \%$ hydrogen peroxide, strip-based bleaching system

Trial design: blinded, randomised controlled trial

Location: not reported

Language: English

Number of centres: 1

Recruitment period: not reported

Funding source: Procter \& Gamble

\section{Participants}

Participants: 19 to 70 years old. Mean age 50 years

Total number: 29

Inclusion criteria:

- healthy subjects with A2 or darker shade

Exclusion criteria:

- any pre-existing oral or medical condition

- crowns or large facial composite restorations on the maxillary anterior teeth

- teeth previously

- current treatment for gingivitis, periodontitis, or caries

- currently using a chlorhexidine or Listerine mouthrinse

- teeth with severe or atypical intrinsic staining

- meaningful malalignment

- fixed orthodontic appliances

Number randomised: 29

Method of randomisation: not reported

Method of allocation concealment: not reported

Method of blinding: not reported

Number evaluated: 28 
Swift 2004 (Continued)

$14 \%$ hydrogen peroxide bleaching strips

Placebo

Duration of treatment: 3 weeks

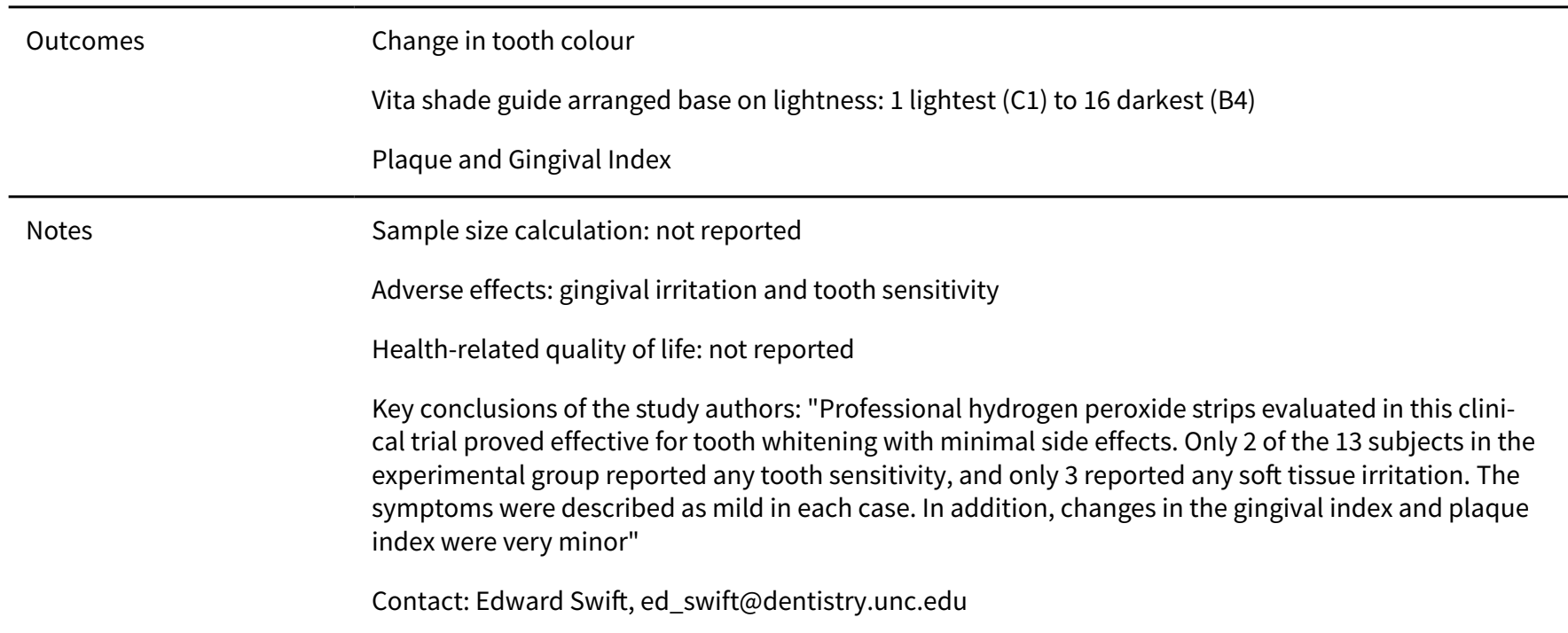

\section{Risk of bias}

Bias Authors' judgement Support for judgement

Random sequence genera- Unclear risk tion (selection bias)

Quote: "Participants were stratified and randomised to treatment groups based on their VITA shade and age." However, the method of randomisation is not mentioned

\begin{tabular}{lll}
\hline $\begin{array}{l}\text { Allocation concealment } \\
\text { (selection bias) }\end{array}$ & Unclear risk & Not mentioned \\
\hline $\begin{array}{l}\text { Blinding of participants } \\
\text { and personnel (perfor- } \\
\text { mance bias) }\end{array}$ & Unclear risk & Not mentioned \\
All outcomes & \\
\hline
\end{tabular}

\begin{tabular}{|c|c|c|}
\hline $\begin{array}{l}\text { Blinding of outcome as- } \\
\text { sessment (detection bias) } \\
\text { All outcomes }\end{array}$ & Unclear risk & Not mentioned \\
\hline \multirow{2}{*}{$\begin{array}{l}\text { Incomplete outcome data } \\
\text { (attrition bias) } \\
\text { All outcomes }\end{array}$} & Low risk & $\begin{array}{l}\text { Quote: "29 subjects were enrolled in the study and received either the experi- } \\
\text { mental or placebo product; } 28 \text { completed the clinical trial" }\end{array}$ \\
\hline & & $\begin{array}{l}\text { Comment: plausible effect size (difference in means) among missing outcomes } \\
\text { not enough to have a clinically relevant impact on observed effect size }\end{array}$ \\
\hline $\begin{array}{l}\text { Selective reporting (re- } \\
\text { porting bias) }\end{array}$ & Low risk & $\begin{array}{l}\text { All outcomes described are reported. Conclusions are in accordance with the } \\
\text { results }\end{array}$ \\
\hline Other bias & Low risk & None \\
\hline
\end{tabular}


Title: effects of duration of whitening strip treatment on tooth colour

Trial design: randomised, double-blinded, placebo-controlled clinical trial

Location: University of North Carolina, USA

Language: English

Number of centres: 1

Recruitment period: not reported

Funding source: Procter \& Gamble (authors are employed by this company)

\title{
Participants
}

Participants: 25 to 58 years

Total number: 40

Inclusion criteria:

- A3 or darker shades

- healthy individuals

Exclusion criteria:

- current tooth sensitivity

- orthodontic treatment

- aesthetic restorations

Number randomised: 40

Method of randomisation: not reported

Method of allocation concealment: not reported

Method of blinding: blinded test kits with similar over packing

Number evaluated: week $2 n=39$, week $4 n=36$, week $6 n=37$ (week 2: 1 dropout from peroxide group; week 4: 1 dropout from peroxide and 3 from placebo; and week 6: 1 dropout from peroxide and 2 dropouts from placebo group)

$6 \%$ hydrogen peroxide gel strips

Placebo

Duration of treatment: 2 weeks

Change in tooth shade
\[ b^{\star}: \text { decreased } b^{\star} \text { indicates reduced yellowness; } \Delta \mathrm{L} \text { : increased } \Delta \mathrm{L} \text { is increased brightness } \]

Notes

\author{
Sample size calculation: not reported \\ Adverse effects: tooth sensitivity and oral irritation \\ Health-related quality of life: not reported
}

Key conclusions of the study authors: "Twice-daily use of $6 \%$ hydrogen peroxide whitening strips resulted in teeth becoming lighter and less yellow versus baseline and placebo during initial 2-week use, with no evidence of placebo response during sustained (weeks 2-6) use"

Contact: Edward Swift, ed_swift@dentistry.unc.edu 
Swift 2009 (Continued)

Risk of bias

\begin{tabular}{|c|c|c|}
\hline Bias & Authors' judgement & Support for judgement \\
\hline $\begin{array}{l}\text { Random sequence genera- } \\
\text { tion (selection bias) }\end{array}$ & Unclear risk & $\begin{array}{l}\text { Quote: "A randomised double-blinded placebo-controlled clinical trial was } \\
\text { conducted... After balancing for starting tooth colour and age, subjects were } \\
\text { randomly assigned to peroxide or placebo strips." However, method of ran- } \\
\text { domisation is not mentioned }\end{array}$ \\
\hline $\begin{array}{l}\text { Allocation concealment } \\
\text { (selection bias) }\end{array}$ & Unclear risk & Not mentioned \\
\hline $\begin{array}{l}\text { Blinding of participants } \\
\text { and personnel (perfor- } \\
\text { mance bias) } \\
\text { All outcomes }\end{array}$ & Low risk & $\begin{array}{l}\text { Placebo strips were identical to the test strips. Quote: "All test products and in- } \\
\text { structions for use were packaged in a blinded test kit.." }\end{array}$ \\
\hline $\begin{array}{l}\text { Blinding of outcome as- } \\
\text { sessment (detection bias) } \\
\text { All outcomes }\end{array}$ & Unclear risk & Not mentioned \\
\hline $\begin{array}{l}\text { Incomplete outcome data } \\
\text { (attrition bias) } \\
\text { All outcomes }\end{array}$ & Low risk & $\begin{array}{l}\text { Quote: "1 subject in peroxide group was dismissed early as a recall failure. } \\
4 \text { other subjects completed the study but were excluded from the statistical } \\
\text { analysis because of missed visits or non-compliance" } \\
\text { Comment: missing outcome data balanced in numbers across intervention } \\
\text { groups }\end{array}$ \\
\hline $\begin{array}{l}\text { Selective reporting (re- } \\
\text { porting bias) }\end{array}$ & Low risk & $\begin{array}{l}\text { All outcomes discussed have been reported adequately. Conclusions are in ac- } \\
\text { cordance with the results }\end{array}$ \\
\hline Other bias & Low risk & Outliers in the data sets are removed. It may have an effect on the results \\
\hline
\end{tabular}

Tam 2001

Title: effect of potassium nitrate and fluoride on carbamide peroxide bleaching
Trial design: split-mouth, double-blinded, randomised clinical trial
Location: University of Toronto, Canada
Language: English
Number of centres: 1
Recruitment period: not reported
Funding source: not reported

Participants

\author{
Participants: 20 to 53 years old with a mean age of 31 (10) years \\ Total number: 42 \\ Inclusion criteria: not reported \\ Exclusion criteria: no previous history of desensitizing agents \\ Number randomised: not reported
}


Tam 2001 (Continued)

Method of randomisation: not reported

Method of allocation concealment: syringes were randomly numbered and selected for use

Method of blinding: identical packs

Number evaluated: 40

\begin{tabular}{ll}
\hline Interventions & Total number of intervention groups: 2 \\
$10 \%$ carbamide peroxide with $3 \%$ potassium nitrate and 0.11 fluoride ion wt/vol \\
$10 \%$ carbamide peroxide \\
Duration of treatment: 2 weeks \\
\hline Tooth sensitivity \\
Tooth whitening: patient-reported improvement \\
Sample size calculation: not reported \\
Adverse effects: sensitivity \\
Key conclusions of the study authors: "A 10\% carbamide peroxide bleaching gel containing potassium \\
nitrate and fluoride produced less tooth sensitivity than did the control bleaching gel during a 2-week \\
at-home bleaching treatment" \\
Correspondence required: no \\
Contact: Dr Laura Tam, laura.tam@utotonto.ca
\end{tabular}

\section{Risk of bias}

Bias Authors' judgement Support for judgement

Random sequence genera- Unclear risk Quote: "double-blinded randomised clinical trial." However, method of rantion (selection bias) domisation is not reported

\begin{tabular}{l}
$\begin{array}{l}\text { Allocation concealment } \begin{array}{l}\text { Unclear risk } \\
\text { (selection bias) }\end{array} \\
\begin{array}{l}\text { Quote: "syringes were randomly numbered and selected for use on either the } \\
\text { left or right side of each patient's dental arch" }\end{array} \\
\text { Comment: we are not sure if the person allocating the participants and the } \\
\text { person conducting the study are the same }\end{array}$ \\
\hline
\end{tabular}

Blinding of participants Low risk Quote: "formulations were manufactured specifically for this study (Ultradent)
and personnel (perfor- $\quad$ and were packaged identically for..."

mance bias)

All outcomes

\begin{tabular}{|c|c|c|}
\hline $\begin{array}{l}\text { Blinding of outcome as- } \\
\text { sessment (detection bias) } \\
\text { All outcomes }\end{array}$ & Unclear risk & $\begin{array}{l}\text { Quote: "double-blinded randomised clinical trial." However, method of blind- } \\
\text { ing is not reported }\end{array}$ \\
\hline
\end{tabular}

$\begin{array}{ll}\begin{array}{l}\text { Incomplete outcome data } \\ \text { (attrition bias) }\end{array} & \text { Unclear risk } \\ \begin{array}{l}\text { All outcomes } \\ \text { Cuote: "1 patient chose to discontinue treatment .... A total of } 9 \text { treatment } \\ \text { days (out of a potential total of } 294 \text { treatment days) were missed by the pa- } \\ \text { tients, either because of general tooth sensitivity or for personal reasons" }\end{array} \\ \text { Comment: no clear mention of dropouts in the article }\end{array}$


Tam 2001 (Continued)

$\begin{array}{ll}\begin{array}{l}\text { Selective reporting (re- } \quad \text { Low risk } \\ \text { porting bias) }\end{array} & \begin{array}{l}\text { All outcomes discussed have been reported adequately. Conclusions are in ac- } \\ \text { cordance with the results }\end{array}\end{array}$

\begin{tabular}{lll}
\hline Other bias $\quad$ Low risk None &
\end{tabular}

Tsubura 2005

Title: clinical evaluation of a new bleaching product Polanight in a Japanese population
Trial design: split-mouth, randomised controlled trial
Location: private dental clinic
Language: English
Number of centres: 1
Recruitment period: not reported
Funding source: SDI Ltd

Participants

Participants: 18 to 47 years old. Mean age 30 years

Total number: 58

Inclusion criteria: not reported

Exclusion criteria: not reported

Number randomised: 58

Method of randomisation: not reported

Method of allocation concealment: not reported

Method of blinding: not reported

Number evaluated: 58

Interventions

Total number of intervention groups: 2

Polanight: $10 \%$ carbamide peroxide

Opalescence: $10 \%$ carbamide peroxide

Duration of treatment: 2 weeks

\section{Outcomes}

Improvement in tooth colour

$\Delta \mathrm{L}, \mathrm{a}^{\star}, \mathrm{b}^{*}$ values: increase in $\Delta \mathrm{L}$ and decrease in $\mathrm{b}^{*}$ indicates lightening of teeth

Sample size calculation: not reported
Adverse effects: sensitivity
Health-related quality of life: not reported
Key conclusions of the study authors: "Treatment with either agent demonstrated significant bleaching
effects produced by the treatment. Bleaching with PN was considered more effective than that with OP
in the young patient group and in the women"
Correspondence required: no


Tsubura 2005 (Continued)

Contact: R Yamaguchi, hshimo@ngt.ndu.ac.jp

\section{Risk of bias}

\begin{tabular}{|c|c|c|}
\hline Bias & Authors' judgement & Support for judgement \\
\hline $\begin{array}{l}\text { Random sequence genera- } \\
\text { tion (selection bias) }\end{array}$ & Unclear risk & $\begin{array}{l}\text { Quote: "...were randomly selected from the patients visiting." However, } \\
\text { method of randomisation is not described }\end{array}$ \\
\hline $\begin{array}{l}\text { Allocation concealment } \\
\text { (selection bias) }\end{array}$ & Unclear risk & Not mentioned \\
\hline $\begin{array}{l}\text { Blinding of participants } \\
\text { and personnel (perfor- } \\
\text { mance bias) } \\
\text { All outcomes }\end{array}$ & Unclear risk & Not mentioned \\
\hline $\begin{array}{l}\text { Blinding of outcome as- } \\
\text { sessment (detection bias) } \\
\text { All outcomes }\end{array}$ & Unclear risk & Not mentioned \\
\hline $\begin{array}{l}\text { Incomplete outcome data } \\
\text { (attrition bias) } \\
\text { All outcomes }\end{array}$ & Low risk & No dropouts \\
\hline $\begin{array}{l}\text { Selective reporting (re- } \\
\text { porting bias) }\end{array}$ & Low risk & $\begin{array}{l}\text { All outcomes described were reported. Conclusions are in accordance with the } \\
\text { results }\end{array}$ \\
\hline Other bias & Low risk & None \\
\hline
\end{tabular}

Turkun 2010

Methods Title: 1-year clinical evaluation of the efficacy of a new daytime at-home bleaching technique

Trial design: parallel group randomised controlled clinical trial

Location: Ege University, Turkey

Language: English

Number of centres: 1

Recruitment period: not reported

Funding source: no

Participants

Participants: 20 to 30 years old

Total number: 20

Inclusion criteria:

- have all maxillary and mandibular anterior teeth with a shade mean of C1 or darker

- no caries and restoration on the teeth to be bleached

- be between 20 and 30 years old

- be able to return for periodic controls

- consuming the products that stain teeth (coffee, red wine, tea, etc.) not more than 5 times in a day 
Turkun 2010 (Continued)

Exclusion criteria:

- poor general or dental health

- fixed orthodontic appliances

- having hypersensitive teeth

- smoking

- current or previous use of bleaching agents

- pregnant or lactating women

- tetracycline-stained teeth

- a history of allergies to tooth whitening products

Number randomised: 20

Method of randomisation: not reported

Method of allocation concealment: not reported

Method of blinding: not reported

Number evaluated: 20

Total number of intervention groups: 2
$28 \%$ carbamide peroxide gel in daytime non-custom-fit tray (Meta Tray)
$10 \%$ carbamide peroxide gel in overnight custom-fit tray (Opalescence PF)
Duration of treatment: 10 days

Outcomes Improvement in tooth shade: $\Delta \mathrm{L}, \mathrm{a}^{\star}, \mathrm{b}^{\star}$ values: increase in $\Delta \mathrm{L}$ and decrease in $\mathrm{b}^{*}$ indicates improvement

Sensitivity: 0 - no changes noted, 1 - mild sensitivity, 2 - moderate sensitivity, and 3 - severe sensitivity

\section{Notes}

Sample size calculation: not mentioned

Adverse effects: sensitivity

Health-related quality of life: not mentioned

Key conclusions of the study authors: "...daytime at-home bleaching system tested (Meta Tray) produced significant bleaching effects. However, the clinical efficacy of the overnight bleaching system was found superior to this new daytime at-home bleaching system. Al though the new bleaching system exhibited less tooth sensitivity probably because of the reduced contact time of the bleaching gel with tooth surfaces, the application of the bleaching agent with a non-customized tray provoked more gingival sensitivity in this group. The whitening effect remained similar 1 year after the bleaching"

\section{Risk of bias}

\section{Bias \\ Authors' judgement Support for judgement}

Random sequence genera- Unclear risk tion (selection bias)

Quote: "20 adult subjects ......were selected to participate in this randomised, controlled clinical trial." However, method of randomisation is not mentioned

\begin{tabular}{lll}
\hline $\begin{array}{l}\text { Allocation concealment } \\
\text { (selection bias) }\end{array}$ & Unclear risk mentioned \\
\hline
\end{tabular}

\begin{tabular}{|c|c|c|}
\hline $\begin{array}{l}\text { Blinding of participants } \\
\text { and personnel (perfor- }\end{array}$ & High risk & $\begin{array}{l}1 \text { group had a custom-made tray while the other used a prefabricated tray. } \\
\text { There is a high risk that participants get to know the difference }\end{array}$ \\
\hline
\end{tabular}
mance bias)

All outcomes 
Turkun 2010 (Continued)

$\begin{aligned} & \text { Blinding of outcome as- } \\ & \text { sessment (detection bias) }\end{aligned} \quad$ Low risk $\quad \begin{aligned} & \text { Quote: "...by an examiner who did not know the treatment details of the pa- } \\ & \text { tients" }\end{aligned}$

All outcomes

\begin{tabular}{lll}
$\begin{array}{l}\text { Incomplete outcome data } \\
\text { (attrition bias) } \\
\text { All outcomes }\end{array}$ & Low risk & Quote: "All 20 participants completed this study" \\
\hline $\begin{array}{l}\text { Selective reporting (re- } \\
\text { porting bias) }\end{array}$ & Low risk & $\begin{array}{l}\text { All outcomes described have been reported. Conclusions are in accordance } \\
\text { with the results }\end{array}$ \\
\hline Other bias & Low risk & None
\end{tabular}

Walters 2004

\section{Methods}

Title: benefits of sodium hexametaphosphate-containing chewing gum for extrinsic stain inhibition

Trial design: placebo-controlled, randomised, examiner-blinded, 2-period cross-over trial

Location: London, UK

Language: English

Number of centres: 1

Recruitment period: not reported

Funding source: author is employed by Procter \& Gamble

Total number: 11

Inclusion criteria:

- 18 years old

- minimum of 16 natural teeth, including at least 7 of the 8 anterior incisor teeth

Exclusion criteria:

- known hypersensitivity to chlorhexidine digluconate or polyphosphates

- anterior facial restorations

- evidence of temporomandibular joint dysfunction

- presence of oral ulcers, self-reported diabetes

- self-reported pregnancy

Number randomised: 11

Method of randomisation: not reported

Method of allocation concealment: not reported

Method of blinding: not reported

Number evaluated: 10. 1 dropout from the experimental (chewing gum first/negative control gum second) due to an adverse event

$\begin{array}{ll}\text { Interventions } & \text { Total number of intervention groups: } 2 \\ & \text { Experimental: } 5.6 \% \text { sodium hexametaphosphate chewing gum }\end{array}$


Walters 2004 (Continued)

Control: non-sodium hexametaphosphate chewing gum

Duration of treatment: 3 days, 10 days washout period before cross-over

\begin{tabular}{ll}
\hline Outcomes & Reduction in induced extrinsic stains \\
\hline Notes & Adverse effects: not reported \\
& Health-related quality of life: not reported \\
& Key conclusions of the study authors: "Sodium hexametaphosphate-containing chewing gum can sig- \\
nificantly reduce induced extrinsic dental stain formation, compared to a non-sodium hexametaphos- \\
phate chewing gum" \\
Contact: Patricia A Walters, walters.pa@pg.com
\end{tabular}

\section{Risk of bias}

\begin{tabular}{|c|c|c|}
\hline Bias & Authors' judgement & Support for judgement \\
\hline $\begin{array}{l}\text { Random sequence genera- } \\
\text { tion (selection bias) }\end{array}$ & Unclear risk & $\begin{array}{l}\text { Quote: "The study was a negative-controlled, examiner-blinded, randomised, } \\
\text { 2-period cross-over design." However, method of randomisation is not men- } \\
\text { tioned }\end{array}$ \\
\hline $\begin{array}{l}\text { Allocation concealment } \\
\text { (selection bias) }\end{array}$ & Unclear risk & Not mentioned \\
\hline $\begin{array}{l}\text { Blinding of participants } \\
\text { and personnel (perfor- } \\
\text { mance bias) } \\
\text { All outcomes }\end{array}$ & Unclear risk & $\begin{array}{l}\text { Quote: "The study was a negative-controlled, examiner-blinded, randomised, } \\
\text { 2-period cross-over design." However, method of blinding is not mentioned }\end{array}$ \\
\hline $\begin{array}{l}\text { Blinding of outcome as- } \\
\text { sessment (detection bias) } \\
\text { All outcomes }\end{array}$ & Unclear risk & $\begin{array}{l}\text { Quote: "The study was a negative-controlled, examiner-blinded, randomised, } \\
\text { 2-period cross-over design." However, method of blinding is not mentioned }\end{array}$ \\
\hline $\begin{array}{l}\text { Incomplete outcome data } \\
\text { (attrition bias) } \\
\text { All outcomes }\end{array}$ & Low risk & $\begin{array}{l}\text { Quote: "1 subject in the experimental sequence dropped from the study due to } \\
\text { an adverse event, reported and diagnosed by the examiner" } \\
\text { Comment: plausible effect size (difference in means) among missing outcomes } \\
\text { not enough to have a clinically relevant impact on observed effect size }\end{array}$ \\
\hline $\begin{array}{l}\text { Selective reporting (re- } \\
\text { porting bias) }\end{array}$ & Low risk & $\begin{array}{l}\text { All outcomes mentioned are reported adequately. Conclusions are in accor- } \\
\text { dance with results }\end{array}$ \\
\hline Other bias & Low risk & None \\
\hline
\end{tabular}

Wong 2004

Methods

Title: randomised controlled trial of home tooth whitening products

Trial design: randomised, double-blinded, single-centre clinical trial with 3 parallel groups

Location: Hong Kong University

Language: English

Number of centres: 1 
Wong 2004 (Continued)

Recruitment period: not reported

Funding source: none

\begin{tabular}{|c|c|}
\hline Participants & $\begin{array}{l}\text { Participants: } 18 \text { to } 30 \text { years old } \\
\text { Total number: } 157 \\
\text { Inclusion criteria: } \\
\text { - } 18 \text { to } 30 \text { years of age and in good general health } \\
\text { - wanted to have their teeth whitened } \\
\text { - had at least } 3 \text { anterior maxillary teeth with tooth shade of Vita A2 } \\
\text { Exclusion criteria: } \\
\text { - had teeth previously bleached } \\
\text { - had dental restorations in the maxillary anterior teeth } \\
\text { - had dental work planned or were currently having dental treatment } \\
\text { cycline stains) } \\
\text { Number randomised: } 87 \\
\text { Method of randomisation: not reported } \\
\text { Method of allocation concealment: not reported } \\
\text { Method of blinding: brands covered with foil } \\
\text { Number evaluated: } 63\end{array}$ \\
\hline Interventions & $\begin{array}{l}\text { Total number of intervention groups: } 3 \\
6 \% \text { hydrogen peroxide strip. Twice daily for } 2 \text { weeks } \\
\text { 18\% carbamide peroxide paint-on gel } \\
\text { Placebo: non-whitening toothpaste } \\
\text { Duration of treatment: } 2 \text { weeks }\end{array}$ \\
\hline Outcomes & $\begin{array}{l}\text { Improvement in tooth whitening: } \Delta \mathrm{L}, \mathrm{a}^{\star}, \mathrm{b}^{\star} \text { : increase in } \Delta \mathrm{L} \text { and reduction in } \mathrm{b}^{\star} \text { indicates whitening } \\
\text { Satisfaction of tooth whitening: 9-point Likert scale }\end{array}$ \\
\hline Notes & $\begin{array}{l}\text { Sample size calculation: none } \\
\text { Adverse effects: irritation of gums or teeth } \\
\text { Health-related quality of life: } 0 \text { to } 4 \text { (never - hardly ever - occasionally - fairly often - often), Oral Health } \\
\text { Impact Profile } \\
\text { Key conclusions of the study authors: "Crest Whitestrips ( } 6.5 \% \text { hydrogen peroxide) and Colgate Simply } \\
\text { White ( } 18 \% \text { carbamide peroxide) are both effective in tooth whitening with the former being more ef- } \\
\text { fective" } \\
\text { Correspondence required: no } \\
\text { Contact: not reported }\end{array}$ \\
\hline
\end{tabular}

\section{Risk of bias}


Wong 2004 (Continued)

Bias Authors' judgement Support for judgement

Random sequence genera- Unclear risk
tion (selection bias)

Quote: "Subject inclusion and exclusion criteria, and randomly allocated into 1 of the following 3 groups." However, method of randomisation is not mentioned

\begin{tabular}{|c|c|c|}
\hline $\begin{array}{l}\text { Allocation concealment } \\
\text { (selection bias) }\end{array}$ & Unclear risk & Not mentioned \\
\hline $\begin{array}{l}\text { Blinding of participants } \\
\text { and personnel (perfor- } \\
\text { mance bias) } \\
\text { All outcomes }\end{array}$ & Low risk & $\begin{array}{l}\text { Quote: "brands of all study products were masked by covering the products } \\
\text { with adhesive aluminium foils" }\end{array}$ \\
\hline $\begin{array}{l}\text { Blinding of outcome as- } \\
\text { sessment (detection bias) } \\
\text { All outcomes }\end{array}$ & Unclear risk & $\begin{array}{l}\text { Quote: "randomised controlled, double-blinded, single-center clinical trial." } \\
\text { However, method of blinding is not reported }\end{array}$ \\
\hline $\begin{array}{l}\text { Incomplete outcome data } \\
\text { (attrition bias) } \\
\text { All outcomes }\end{array}$ & Low risk & $\begin{array}{l}\text { Quote: "Each study group started with } 29 \text { subjects and the } 3 \text { groups W, S, and P } \\
\text { ended with 22, } 21 \text { and } 20 \text { subjects respectively" } \\
\text { Comment: missing outcome data balanced in numbers across intervention } \\
\text { groups }\end{array}$ \\
\hline $\begin{array}{l}\text { Selective reporting (re- } \\
\text { porting bias) }\end{array}$ & Low risk & $\begin{array}{l}\text { All outcomes described were reported. Conclusions are in accordance with the } \\
\text { results }\end{array}$ \\
\hline Other bias & Low risk & None \\
\hline
\end{tabular}

Trial design: randomised, examiner-blinded, placebo-controlled study

Location: Shanghai Second Medical University, Shanghai, China

Language: English

Number of centres: 1

Recruitment period: not reported

Funding source: Procter \& Gamble

\section{Participants}

Participants: 18 to 45 years old. Mean age 21.85 years. $92 \%$ females.

Total number: 52

Inclusion criteria: not reported

Exclusion criteria: not reported

Number randomised: 52

Method of randomisation: not reported

Method of allocation concealment: not reported

Method of blinding: similar kits 
Xu 2007 (Continued)

Number evaluated: 49

Total number of intervention groups: 3
Whitening strips $6 \%$ hydrogen peroxide $(\mathrm{n}=18)$,
Paint-on gel $5.8 \%$ hydrogen peroxide $(\mathrm{n}=17 ; 2$ dropouts)
Negative control (water rinse) ( $\mathrm{n}=17 ; 1$ dropout)
Duration of treatment: 8 days for whitening strip, 15 days for gel and water rinse

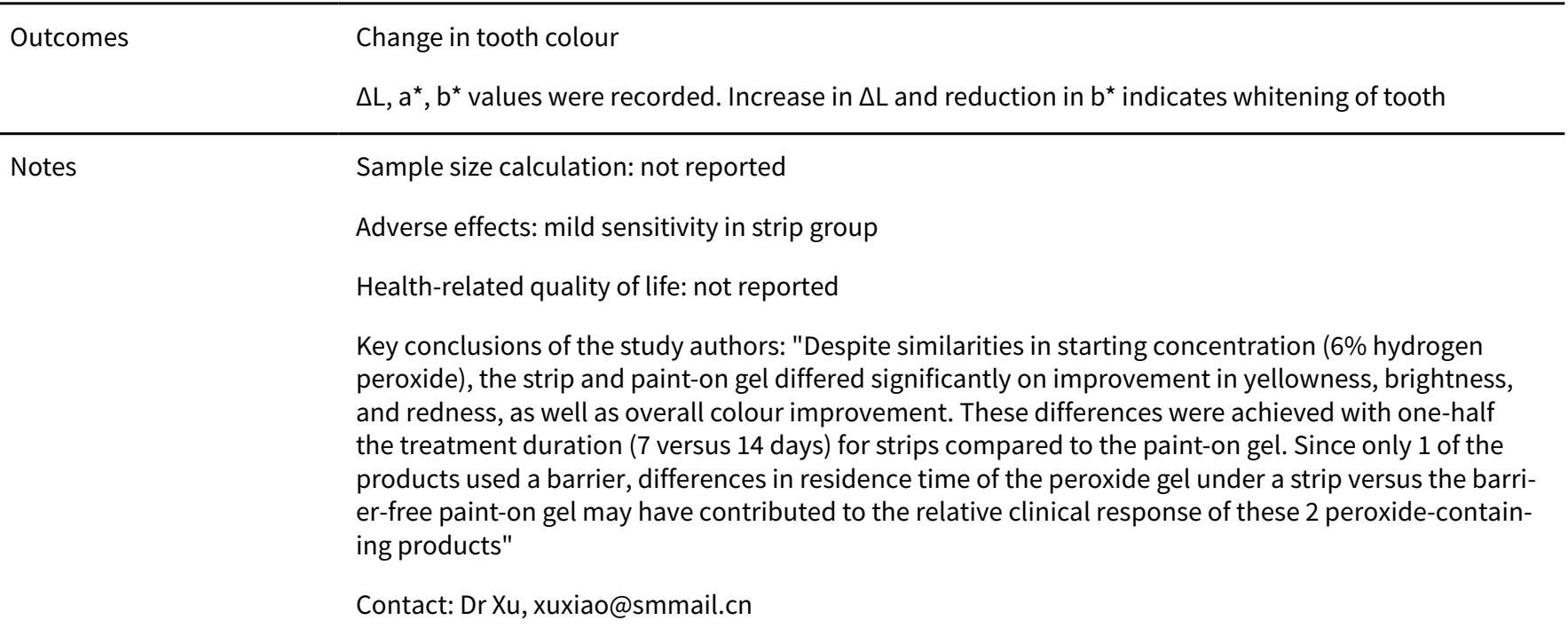

\section{Risk of bias}

\begin{tabular}{|c|c|c|}
\hline Bias & Authors' judgement & Support for judgement \\
\hline $\begin{array}{l}\text { Random sequence genera- } \\
\text { tion (selection bias) }\end{array}$ & Unclear risk & $\begin{array}{l}\text { Quote: "subjects were randomly assigned to peroxide whitening strips (the } \\
\text { positive control), paint-on peroxide whitening gel, or water (the negative con- } \\
\text { trol)." However, method of randomisation is not mentioned }\end{array}$ \\
\hline $\begin{array}{l}\text { Allocation concealment } \\
\text { (selection bias) }\end{array}$ & Unclear risk & Not mentioned \\
\hline $\begin{array}{l}\text { Blinding of participants } \\
\text { and personnel (perfor- } \\
\text { mance bias) } \\
\text { All outcomes }\end{array}$ & Low risk & $\begin{array}{l}\text { Quote: "Subjects were provided an identically appearing kit box labelled only } \\
\text { with the unique subject number" }\end{array}$ \\
\hline $\begin{array}{l}\text { Blinding of outcome as- } \\
\text { sessment (detection bias) } \\
\text { All outcomes }\end{array}$ & Unclear risk & Not mentioned \\
\hline $\begin{array}{l}\text { Incomplete outcome data } \\
\text { (attrition bias) } \\
\text { All outcomes }\end{array}$ & Low risk & $\begin{array}{l}\text { Quote: "All } 18 \text { subjects in the strip group completed the research, while } 2 \text { sub- } \\
\text { jects in the paint-on group and } 1 \text { subject in the water rinse group failed to } \\
\text { complete the study" } \\
\text { Comment: plausible effect size (difference in means) among missing outcomes } \\
\text { not enough to have a clinically relevant impact on observed effect size }\end{array}$ \\
\hline $\begin{array}{l}\text { Selective reporting (re- } \\
\text { porting bias) }\end{array}$ & Low risk & $\begin{array}{l}\text { All outcomes described were reported. Conclusions are in accordance with the } \\
\text { results }\end{array}$ \\
\hline
\end{tabular}


Xu 2007 (Continued)
Other bias
Low risk
None

Ziebolz 2007

Title: efficacy and oral side effects of 2 highly concentrated tray-based bleaching systems
Trial design: randomised, 2-armed, parallel clinical study
Location: University of Göttingen, Germany
Number of centres: 1
Recruitment period: not reported
Funding source: study supported by Kettenbach, Germany
Participants
Participants: 20 to 48 years old
Total number: 60
Inclusion criteria:
- restoration or caries free teeth
- Vita shade score of A2 or darker
- no crowns on upper cuspids or incisors
Exclusion criteria:
- tooth hypersensitivities
- anterior restorations
- poor oral hygiene
- generalised gingival recession
- caries, heavy structural alteration of the tooth structure
- tetracycline or fluorosis staining
Number randomised: 60
Method of randomisation: stratified, randomised distribution
Method of allocation concealment: not reported
Method of blinding: not reported
Number evaluated: 56 (4 dropouts) - three dropouts in VW due to therapy pain and one dropout in OP due to therapy pain.

Interventions

Total number of intervention groups: 2

$7.5 \%$ hydrogen peroxide gel in tray (Visalys)

$20 \%$ carbamide peroxide gel in tray (Opalescence)

Duration of treatment: 12 days

Outcomes

Tooth colour change: $\mathrm{L}^{\star}, \mathrm{a}^{\star}, \mathrm{b}^{\star}$ values: increase in $\mathrm{L}^{\star}$ and decrease in $\mathrm{b}^{\star}$ indicated lightening of teeth

Hypersensitivity: 0 to 10 ( 0 = no hypersensitivity, 10 = high hypersensitivity)

Acceptability: comfortable, slightly disturbing, uncomfortable or very uncomfortable 
Ziebolz 2007 (Continued)

Notes
Sample size calculation: not reported

Adverse effects: sensitivity

Health-related quality of life: not reported

Key conclusions of the study authors: "The bleaching systems demonstrated significant tooth colour improvement for $\Delta \mathrm{b}^{*}$ and $\Delta \mathrm{L}^{*}$. They did produce significantly different whitening response for $\Delta \mathrm{b}^{\star}$, with Opalescence showing significant higher $\Delta \mathrm{b}^{\star}$. After bleaching therapy, the intensity of tooth hypersensitivity was increased significantly compared to baseline, with no significant difference between both groups"

Contact: Dr Dirk Ziebolz, dirk.ziebolz@zm-goettingen.de

\section{Risk of bias}

\begin{tabular}{|c|c|c|}
\hline Bias & Authors' judgement & Support for judgement \\
\hline $\begin{array}{l}\text { Random sequence genera- } \\
\text { tion (selection bias) }\end{array}$ & Unclear risk & $\begin{array}{l}\text { Quote: "A stratified, randomised distribution of the subjects to the } 2 \text { treatment } \\
\text { groups..." However, method is not reported }\end{array}$ \\
\hline $\begin{array}{l}\text { Allocation concealment } \\
\text { (selection bias) }\end{array}$ & Unclear risk & Not mentioned \\
\hline $\begin{array}{l}\text { Blinding of participants } \\
\text { and personnel (perfor- } \\
\text { mance bias) } \\
\text { All outcomes }\end{array}$ & Unclear risk & Not mentioned \\
\hline $\begin{array}{l}\text { Blinding of outcome as- } \\
\text { sessment (detection bias) } \\
\text { All outcomes }\end{array}$ & Unclear risk & Not mentioned \\
\hline $\begin{array}{l}\text { Incomplete outcome data } \\
\text { (attrition bias) } \\
\text { All outcomes }\end{array}$ & Low risk & $\begin{array}{l}\text { Quote: "3 subjects from the Visalys group and } 1 \text { from the Opalescence group } \\
\text { withdrew during bleaching therapy..." } \\
\text { Comment: missing outcome data balanced in numbers across intervention } \\
\text { groups }\end{array}$ \\
\hline $\begin{array}{l}\text { Selective reporting (re- } \\
\text { porting bias) }\end{array}$ & Low risk & $\begin{array}{l}\text { All outcomes described were reported. Conclusions are in accordance with the } \\
\text { results }\end{array}$ \\
\hline Other bias & Low risk & None \\
\hline
\end{tabular}

Ziebolz 2008

Title: influence of a desensitizing agent on efficacy of a paint-on bleaching agent
Trial design: double-blinded, randomised controlled trial
Location: University of Göttingen, Germany
Language: English
Number of centres: 1
Recruitment period: not reported




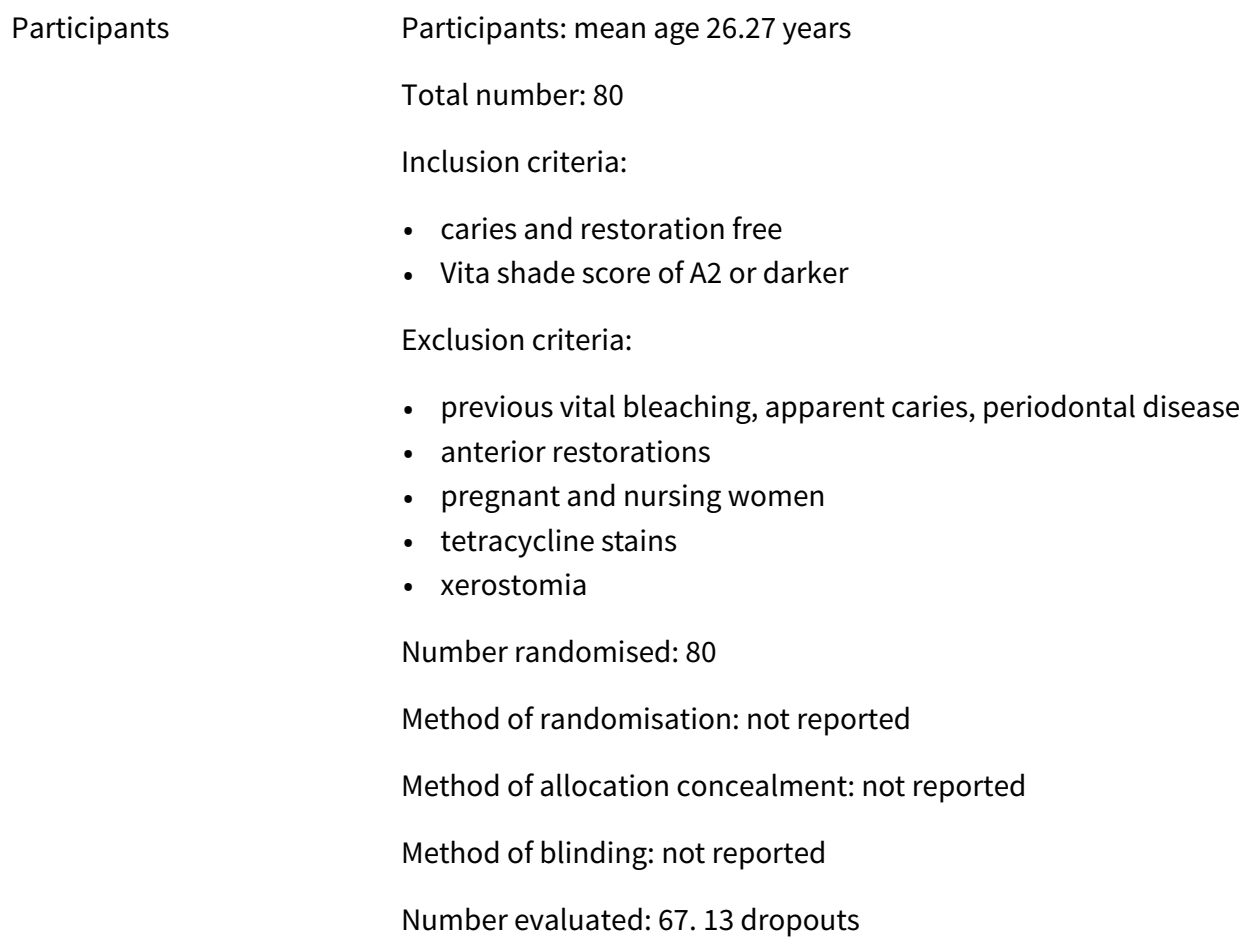

Participants

Participants: mean age 26.27 years

Total number: 80

Inclusion criteria:

- caries and restoration free

- Vita shade score of A2 or darker

Exclusion criteria:

- previous vital bleaching, apparent caries, periodontal disease

- anterior restorations

- pregnant and nursing women

- tetracycline stains

- xerostomia

Number randomised: 80

Method of randomisation: not reported

Method of allocation concealment: not reported

Method of blinding: not reported

Number evaluated: 67.13 dropouts

Total number of intervention groups: 2

Control: hydrogen peroxide $6 \%$ without desensitizers ( $n=40$, final 7 dropouts)

Experimental: hydrogen peroxide $6 \%$ with desensitizer ( $n=40$, final 6 dropouts)

Duration of treatment: 10 days

\section{Outcomes}

Tooth colour change: Vita shade guide

Tooth hypersensitivity: $0=$ no sensitivity, $10=$ high sensitivity

Acceptability: interview: comfortable, slightly disturbing, uncomfortable or very uncomfortable

Tolerability: interview: comfortable, slightly disturbing, uncomfortable or very uncomfortable

Adverse effects: sensitivity and irritation in both groups

Key conclusions of the study authors: "treatment groups developed tooth hypersensitivities during bleaching therapy. The number of subjects exhibiting sensitivities after bleaching increased more in the group without application of VivaSens (plus 15.2\%) than in the group with VivaSens (2.9\%), but the difference between the groups was not significant. Lack of statistical significance might be due to the low increase of tooth sensitivities due to the bleaching. This relatively low incidence of tooth hypersensitivity might be explained by the low concentration of peroxide $(6.0 \%)$ in the bleaching agent used in this study. It might be speculated that a distinct higher number of subjects with tooth hypersensitivities due the bleaching therapy in the control group (Group A) without VivaSens application might have better demonstrated the positive effect of the desensitizing agent"

Contact: Dr Dirk Ziebolz, dirk.ziebolz@zm-goettingen.de

\section{Risk of bias}


Ziebolz 2008 (Continued)

\begin{tabular}{|c|c|c|}
\hline Bias & Authors' judgement & Support for judgement \\
\hline $\begin{array}{l}\text { Random sequence genera- } \\
\text { tion (selection bias) }\end{array}$ & Unclear risk & $\begin{array}{l}\text { Quote: "The } 80 \text { subjects were distributed randomly among } 2 \text { groups" However, } \\
\text { method of randomisation is not mentioned }\end{array}$ \\
\hline $\begin{array}{l}\text { Allocation concealment } \\
\text { (selection bias) }\end{array}$ & Unclear risk & Not mentioned \\
\hline $\begin{array}{l}\text { Blinding of participants } \\
\text { and personnel (perfor- } \\
\text { mance bias) } \\
\text { All outcomes }\end{array}$ & Unclear risk & $\begin{array}{l}\text { Quote: "double-blinded, randomised, controlled, parallel-group clinical trial." } \\
\text { However, method of blinding is not reported }\end{array}$ \\
\hline $\begin{array}{l}\text { Blinding of outcome as- } \\
\text { sessment (detection bias) } \\
\text { All outcomes }\end{array}$ & Unclear risk & $\begin{array}{l}\text { Quote: "double-blinded, randomised, controlled, parallel-group clinical trial." } \\
\text { However, method of blinding is not reported }\end{array}$ \\
\hline $\begin{array}{l}\text { Incomplete outcome data } \\
\text { (attrition bias) } \\
\text { All outcomes }\end{array}$ & Low risk & $\begin{array}{l}\text { Quote: "13 subjects failed to complete the } 2 \text {-week study ( } 1 \text { from each group) } \\
\text { for reasons unrelated to the study } \\
\text { Comment: missing outcome data balanced in numbers across intervention } \\
\text { groups }\end{array}$ \\
\hline $\begin{array}{l}\text { Selective reporting (re- } \\
\text { porting bias) }\end{array}$ & Low risk & $\begin{array}{l}\text { All outcomes described were reported. Conclusions are in accordance with the } \\
\text { results }\end{array}$ \\
\hline Other bias & Low risk & None \\
\hline
\end{tabular}

$\mathrm{CP}=$ carbamide peroxide; $\mathrm{HP}=$ hydrogen peroxide; $\mathrm{NGVB}=$ nightguard vital bleaching; $\mathrm{SE}=$ standard error; STPP = sodium tripolyphosphate; $\mathrm{VAS}=$ visual analogue scale; $\mathrm{WIO}=$ whiteness index.

Characteristics of excluded studies [ordered by study ID]

\begin{tabular}{ll}
\hline Study & Reason for exclusion \\
\hline Alkmin 2005 & Study compared the effects of 2 bleaching agents on oral microbiota. \\
\hline Amini 2009 & Abstract: insufficient information to include. \\
\hline Amini 2011 & Abstract: insufficient information to include. \\
\hline Anastasia 2010 & Abstract: insufficient information to include. \\
\hline Andreana 2000 & Abstract: insufficient information to include. \\
\hline Archila 2010 & Abstract: insufficient information to include. \\
\hline Auschill 2009 & Abstract: insufficient information to include. \\
\hline Browning 2001 & Abstract: insufficient information to include. \\
\hline Burgio 2001 & Study involved in-office bleaching as intervention. \\
\hline Cardoso 2010 & $\begin{array}{l}\text { Study compared the clinical effectiveness and tooth sensitivity associated with different bleaching } \\
\text { agents in children. }\end{array}$ \\
\hline
\end{tabular}




\begin{tabular}{ll}
\hline Study & Reason for exclusion \\
\hline Collins 2004 & Study compared the effect of self-applied tooth whitening gel on oral soft tissue. \\
\hline Corby 2014 & Study compared the effect of hydrogen peroxide whitening strips in adolescent twins. \\
\hline Curtis 1996 & Study compared the effect of carbamide peroxide whitening gel on oral soft tissue. \\
\hline de Geus 2015a & $\begin{array}{l}\text { Controlled clinical trial evaluating the genotoxicity and efficacy of at-home bleaching in smokers } \\
\text { and non-smokers. }\end{array}$ \\
\hline de Geus $2015 b$ & Controlled clinical trial evaluating tooth sensitivity in smokers. \\
\hline Dickinson 2000 & Abstract: insufficient information to include. \\
\hline Donly 2001 & Abstract: insufficient information to include. \\
\hline Donly 2002 & Study compared the use of whitening strips in children. \\
\hline Donly $2002 a$ & Study compared the use of whitening strips in children and adolescents. \\
\hline Farrell 2006 & The home-based bleach was applied professionally in the clinic. \\
\hline Farrell 2008 & $\begin{array}{l}\text { Study compared the effect of hydrogen peroxide whitening strips on tooth sensitivity and oral irri- } \\
\text { tation. }\end{array}$
\end{tabular}

\begin{tabular}{|c|c|}
\hline Fugaro 2004 & Study evaluated the histological changes in dental pulp after nightguard vital bleaching. \\
\hline Fugaro 2005 & Study assessed the expression of specific neuropeptides associated with inflammation. \\
\hline Garcia-Godoy 2012 & Abstract: insufficient information to include. \\
\hline Gerlach 2002c & The intervention included toothbrushing. \\
\hline Gerlach 2002d & Abstract: insufficient information to include. \\
\hline Gerlach 2002e & Study compared 2 professional tooth whitening systems for shade change using chroma meter. \\
\hline Gerlach 2003a & One of the interventions included a whitening dentifrice. \\
\hline Gerlach 2004a & One of the interventions included a whitening dentifrice. \\
\hline Gerlach 2004c & Study evaluated the peroxide degradation and dilution kinetics of a bleaching agent. \\
\hline Gerlach 2004d & Trial included teenagers and younger age group participants. \\
\hline Godson 2001 & Abstract: insufficient information to include. \\
\hline Gursoy 2008 & $\begin{array}{l}\text { Study compared the effect of external tooth bleaching on dental plaque accumulation and dis- } \\
\text { colouration. }\end{array}$ \\
\hline Jadad 2011 & $\begin{array}{l}\text { Study evaluated the colour alterations with a new dental bleaching product in patients wearing or- } \\
\text { thodontic appliances. }\end{array}$ \\
\hline Jorgensen 2002 & Study compared the incidence of tooth sensitivity after home whitening treatment. \\
\hline Karpinia 2003 & One of the interventions included a whitening dentifrice. \\
\hline
\end{tabular}




\begin{tabular}{|c|c|}
\hline Study & Reason for exclusion \\
\hline Lee 2003 & Abstract: insufficient information to include. \\
\hline Leonard 2002 & Study evaluated safety issues when using a $16 \%$ carbamide peroxide whitening solution. \\
\hline Leonard 2004 & Study evaluated the efficacy of a desensitizing agent used along with a bleaching agent. \\
\hline Leonard 2007 & $\begin{array}{l}\text { Study evaluated effects on oral tissues and associated tooth sensitivity and patients' perceptions } \\
\text { during tooth bleaching. }\end{array}$ \\
\hline Lisante 2009 & Abstract: insufficient information to include. \\
\hline Loyola-Rodriguez 2003 & Study included adolescents. \\
\hline Majeed 2011 & Abstract: insufficient information to include. \\
\hline Marques 2012 & $\begin{array}{l}\text { Study compared salivary hydrogen peroxide release kinetics and potential toxicity of systemic ex- } \\
\text { posure of } 4 \text { different whitening products. }\end{array}$ \\
\hline Martin 2015 & In-office bleaching was used in intervention. \\
\hline Matis 1999 & Study evaluated in vivo degradation rate of beaching gels in bleaching trays. \\
\hline Matis 2002 & Study compared different degradation of $15 \%$ carbamide peroxide. \\
\hline Matis 2002a & A clinical evaluation of a bleaching agent used with and without reservoirs. \\
\hline Matis 2005 & Quasi-randomised controlled trial. \\
\hline Mazur 2013 & Abstract: insufficient information to include. \\
\hline NCT02603354 & Study involved in-office bleaching as intervention. \\
\hline NCT02682329 & Study involved in-office bleaching as intervention. \\
\hline Perdigao 2013 & Abstract: insufficient information to include. \\
\hline Pinto 2014 & Tooth whitening with hydrogen peroxide in adolescents. \\
\hline Pinto 2017 & Study compared whitening with hydrogen peroxide in adolescents. \\
\hline Rezende 2013 & Study compared the clinical effects of exposure to coffee during at-home vital bleaching. \\
\hline Sagel 2001 & Abstract: insufficient information to include. \\
\hline Schiff 1994 & Study is not about home-based bleaching. \\
\hline Schulte 1993 & Study evaluated clinical changes in the gingiva as a result of at-home bleaching. \\
\hline Schulte 1994 & Study evaluated and compared pulpal responses of teeth exposed to a bleaching agent. \\
\hline Simon 2001 & $\begin{array}{l}\text { Trial comparing tooth whitening with peroxide-containing strips to a marketed whitening denti- } \\
\text { frice. }\end{array}$ \\
\hline Simon 2011 & Abstract: insufficient information to include. \\
\hline
\end{tabular}




\begin{tabular}{ll}
\hline Study & Reason for exclusion \\
\hline Smith 2001 & Abstract: insufficient information to include. \\
\hline Swift 2001 & Abstract: insufficient information to include. \\
\hline Tam 1999 & Study participants included adolescents. \\
\hline Walter 2011 & Abstract: insufficient information to include. \\
\hline Yankell 1997 & Intervention used in the study (chewing gum) is excluded from this review. \\
\hline Zantner 2006 & In-office application was done for 1 group. \\
\hline
\end{tabular}

Characteristics of studies awaiting assessment [ordered by study ID]

\section{Barnes 1998}

\begin{tabular}{|c|c|}
\hline \multirow[t]{7}{*}{ Methods } & Title: clinical evaluation of a new $10 \%$ carbamide peroxide tooth-whitening agent \\
\hline & Trial design: double-blinded clinical trial \\
\hline & Location: University of Maryland Dental School, USA \\
\hline & Language: English \\
\hline & Number of centres: 1 \\
\hline & Recruitment period: not reported \\
\hline & Funding source: Dentsply \\
\hline \multirow[t]{9}{*}{ Participants } & Participants: 18 to 65 years \\
\hline & Total number: 61 \\
\hline & Inclusion criteria: $\mathrm{A} 3$ or darker shade \\
\hline & Exclusion criteria: not reported \\
\hline & Number randomised: not reported \\
\hline & Method of randomisation: not reported \\
\hline & Method of allocation concealment: not reported \\
\hline & Method of blinding: not reported \\
\hline & Number evaluated: 50 \\
\hline \multirow[t]{4}{*}{ Interventions } & Total number of intervention groups: 2 groups \\
\hline & $10 \%$ carbamide peroxide gel in tray \\
\hline & Placebo \\
\hline & Duration of treatment: 4 hours to overnight for 2 weeks \\
\hline \multirow[t]{2}{*}{ Outcomes } & Improvement in tooth shade: 2 weeks, 3 months and 6 months \\
\hline & Tooth sensitivity \\
\hline
\end{tabular}




\section{Gingival irritation}

Notes

Sample size calculation: not reported

Adverse effects: sensitivity

Health-related quality of life: not reported

Key conclusions of the study authors: "The average shade change for the placebo users was less than 1 shade. The average shade change for the NUPRO Gold users was 6.96 shades. Tooth hypersensitivity varied from none to severe. Tissue irritation was minimal. The results of these evaluations indicate that NUPRO Gold is effective as a tooth whitening system, when administered properly under the supervision of a dentist, with commonly reported side effects of transient tooth sensitivity and minimal gingival sensitivity. Little or no change in tissue health was noted"

Correspondence required: yes: unclear if it is a randomised controlled trial. Authors have been mailed requesting for the data

Contact: Dr Douglas Barnes, University of Maryland, Maryland, USA

\section{Bizhang 2017}

Title: effectiveness of a new non-hydrogen peroxide bleaching age
ble-blind placebo-controlled short-term study
Trial design: double-blinded randomised placebo-controlled trial
Location: not reported in abstract
Number of centres: 1
Recruitment period: not reported in abstract
Funding source: not reported in abstract

\section{Participants}

Participants: not reported

Total number: 40

Inclusion criteria: not reported in abstract

Exclusion criteria: not reported in abstract

Number randomised: 40

Method of randomisation: not reported in abstract

Method of allocation concealment: not reported in abstract

Method of blinding: not reported in abstract

Number evaluated: not reported in abstract

Over-the-counter product

Placebo

Duration of treatment: 1 day

Outcomes Improvement in tooth shade


Bizhang 2017 (Continued)
Notes
Yet to procure full text for this article

Braun 2007

Title: spectrophotometric and visual evaluation of vital to
bamide peroxide concentrations
Trial design: double-blinded randomised controlled trial
Location: not reported
Language: English
Number of centres: 1
Recruitment period: not reported
Funding source: not reported

\section{Participants}

Participants: not reported

Total number: 30

Inclusion criteria: not reported

Exclusion criteria: not reported

Number randomised: 30

Method of randomisation: not reported

Method of allocation concealment: not reported

Method of blinding: unmarked syringes

Number evaluated: 30

Total number of intervention groups: 3

$0 \%$ carbamide peroxide

$10 \%$ carbamide peroxide

$17 \%$ carbamide peroxide

Duration of treatment: 1 week

Outcomes Improvement in tooth shade

Notes

Sample size calculation: not reported

Adverse effects: not reported

Key conclusions of the study authors: "The study indicates that higher concentration bleaching agents might whiten teeth faster with major changes in lightness and chroma. However, by bleaching daily for 1 week, similar effects can be achieved with both a high and a low concentration agent. After

treatment, a regression of the resultant shade has to be expected"

Correspondence required: yes: authors have been mailed requesting for missing data 
Browning 2004

\begin{tabular}{|c|c|}
\hline \multirow[t]{2}{*}{ Methods } & $\begin{array}{l}\text { Title: safety and efficacy of a nightguard bleaching agent containing sodium fluoride and potassi- } \\
\text { um nitrate }\end{array}$ \\
\hline & Trial design: unknown \\
\hline \multirow[t]{7}{*}{ Participants } & Participants: 22 \\
\hline & Total number: 22 \\
\hline & Number randomised: not clear \\
\hline & Method of randomisation: not clear \\
\hline & Method of allocation concealment: not clear \\
\hline & Method of blinding: not clear \\
\hline & Number evaluated: 22 \\
\hline \multirow[t]{2}{*}{ Interventions } & $10 \%$ carbamide peroxide with potassium nitrate and sodium fluoride \\
\hline & Placebo \\
\hline \multirow[t]{2}{*}{ Outcomes } & Tooth whitening \\
\hline & Sensitivity of teeth, gingiva, tongue and throat \\
\hline \multirow[t]{4}{*}{ Notes } & Randomisation: not mentioned \\
\hline & Sample size calculation: not reported \\
\hline & Correspondence required: yes: authors have been mailed requesting for missing data \\
\hline & Contact: Dr William Browning, wbrownin@mail.mcg.edu \\
\hline
\end{tabular}

\section{Ferrari 2004}

Trial design: randomised, double-blinded, parallel-group clinical study

Location: private practice, Italy

Language: English

Number of centres: 1

Recruitment period: not reported

Funding source: Procter \& Gamble and Fiji

Participants Participants: not reported

Total number: 37 
Ferrari 2004 (Continued)

Inclusion criteria: not reported

Exclusion criteria: not Reported

Number randomised: 37

Method of randomisation: not reported

Method of allocation concealment: not reported

Method of blinding: similar packing

Number evaluated: only 34 completed the study among them only 32 - day 7, 29 - day 14, 28 - day 28 were present

\begin{tabular}{|c|c|}
\hline \multirow[t]{5}{*}{ Interventions } & Total number of intervention groups: 3 \\
\hline & $1.8 \%$ hydrogen peroxide strips \\
\hline & $3.3 \%$ hydrogen peroxide strips \\
\hline & $5.3 \%$ hydrogen peroxide strips \\
\hline & Duration of treatment: 28 days \\
\hline
\end{tabular}

Outcomes Improvement in tooth shade

Tooth whitening was characterized by decreased $\mathrm{b}^{*}$ (reduction in yellowness) and increased $\Delta \mathrm{L}^{\star}$ (increased brightness)

Notes

Sample size calculation: not reported

Adverse effects: oral irritation

Health-related quality of life: not reported

Key conclusions of the study authors: "Hydrogen peroxide at concentrations ranging from $1.8 \%-5.3 \%$ resulted in significant colour improvement versus baseline as early as Day 7 . There was a concentration-response for reduction in yellowness (delta $b^{\star}$ ) and lightness improvement (delta $L^{\star}$ ) at all time points, favouring the higher concentrations. While the concentration-whitening relationship approached a linear response at Day 7 , continued treatment resulted in incremental colour improvement. All 3 peroxide concentrations were well tolerated, and no subjects discontinued early due to a treatment-related adverse event"

Correspondence required: yes: number of participants per group is not reported, authors have been mailed requesting these data

Contact: Marco Ferrari, Piazza Attias 19, Livorno 57120, Italy, ferrarimar@unisi.it peroxide

Trial design: double-blinded randomised controlled trial

Location: University of Rome, Italy

Language: English

Number of centres: 1 
Gambarini 2004 (Continued)

Recruitment period: not reported

Funding source: not reported

Participants: 21 to 52 years
Total number: 30
Inclusion criteria: Vita shade score of A2 or darker; adults; staining in teeth
Exclusion criteria: not reported
Number randomised: 30
Method of randomisation: not mentioned
Method of allocation concealment: not mentioned
Method of blinding: not reported
Performance/detection bias: not reported
Number evaluated: 30

Interventions

Total number of intervention groups: 2

Hydrogen peroxide gel 5.9\%

Placebo

Duration of treatment: 2 weeks

\begin{tabular}{ll}
\hline Outcomes & Tooth colour change \\
Sensitivity \\
Bleeding on probing \\
Gingival recession \\
\hline
\end{tabular}

Notes

Adverse effects: sensitivity

Health-related quality of life: not reported

Key conclusions of the study authors: "5.9\% hydrogen peroxide was significantly effective in lightening tooth shade. After only 2 weeks, patients enrolled in the study exhibited an overall mean 4.48-shade improvement from baseline, which was significantly greater than placebo group and far exceeded the ADA minimum requirements to claim "clinical efficacy". In the new Colgate Simply White Clear Whitening Gel group, periodontal health (PI and BOP) improved with time overall. Moreover, dentin hypersensitivity did not significantly increase, and all treatments were generally well tolerated"

Correspondence required: yes: missing data, standard deviation cannot be calculated as $P$ values have not been reported in the study. Author has been contacted

Contact: Dr Gianluca Gambarini, ggambarini@tin.it

Trial design: double-blinded randomised controlled trial 
Gegauff 1993 (Continued)

Location: Ohio State University, USA

Language: English

Number of centres: 1

Recruitment period: not reported

Funding source: Ultradent Products, Inc

\begin{tabular}{|c|c|}
\hline \multirow[t]{10}{*}{ Participants } & Participants: 20 to 27 years. Mean age 22 years \\
\hline & Total number: 20 \\
\hline & $\begin{array}{l}\text { Inclusion criteria: no anterior restorations; history of anterior tooth pain or trauma; recurrent gingi- } \\
\text { val lesions or abnormal variation in tooth colour; adults }\end{array}$ \\
\hline & Exclusion criteria: not reported \\
\hline & Number randomised: 20 \\
\hline & Method of randomisation: not mentioned \\
\hline & Method of allocation concealment: not reported \\
\hline & Method of blinding: similar coded syringes \\
\hline & Performance/detection bias: not reported \\
\hline & Number evaluated: control: $n=17$, (3 dropouts) \\
\hline \multirow[t]{4}{*}{ Interventions } & Total number of intervention groups: 2 \\
\hline & Carbamide peroxide $10 \%$ \\
\hline & Placebo gel \\
\hline & Duration of treatment: 8 weeks \\
\hline \multirow[t]{3}{*}{ Outcomes } & $\begin{array}{l}\text { Tooth colour change: } \mathrm{b}^{\star} \text { (decreased } \mathrm{b}^{\star} \text { indicates reduced yellowness); } \Delta \mathrm{L}^{\star} \text { (increased } \Delta \mathrm{L}^{\star} \text { is in- } \\
\text { creased brightness) }\end{array}$ \\
\hline & Gingival Index \\
\hline & Effect on sulcus depth \\
\hline \multirow[t]{6}{*}{ Notes } & Sample size calculation: not reported \\
\hline & Adverse effects: sensitivity \\
\hline & Health-related quality of life: not reported \\
\hline & $\begin{array}{l}\text { Key conclusions of the study authors: "We found that maxillary canines had a higher lightness } \\
\text { change than maxillary incisors. Additionally, the bleaching treatments significantly reduced the } \\
\text { yellowness of all the maxillary anterior teeth. However, this reduction was partially reversed" }\end{array}$ \\
\hline & $\begin{array}{l}\text { Correspondence required: yes: missing data, mean and standard deviation not reported, authors } \\
\text { have been contacted requesting data }\end{array}$ \\
\hline & $\begin{array}{l}\text { Contact: Dr Gegauff, College of Dentistry, Ohio State University, } 305 \text { West 12th Ave, Columbus } \\
432101241 \text {, USA }\end{array}$ \\
\hline
\end{tabular}




\section{Methods}

Title: vital bleaching with a thin peroxide gel: the safety and efficacy of a professional-strength hydrogen peroxide whitening strip

Trial design: double-blinded, randomised controlled trial

Location: not available

Language: English

Number of centres: not available

Recruitment period: not available

Funding source: not available

\begin{tabular}{|c|c|}
\hline \multirow[t]{9}{*}{ Participants } & Participants: adults \\
\hline & Total number: 38 \\
\hline & Inclusion criteria: not available \\
\hline & Exclusion criteria: not available \\
\hline & Number randomised: not available \\
\hline & Method of randomisation: not available \\
\hline & Method of allocation concealment: not available \\
\hline & Method of blinding: not available \\
\hline & Number evaluated: not available \\
\hline \multirow[t]{4}{*}{ Interventions } & Total number of intervention groups: 2 \\
\hline & $6 \%$ hydrogen peroxide \\
\hline & $14 \%$ hydrogen peroxide \\
\hline & Duration of treatment: 2 weeks \\
\hline Outcomes & Improvement in tooth shade \\
\hline \multirow[t]{6}{*}{ Notes } & Sample size calculation: not available \\
\hline & Adverse effects: oral irritation \\
\hline & Health-related quality of life: not available \\
\hline & $\begin{array}{l}\text { Key conclusions of the study authors: "Use of the thin } 14 \% \text { hydrogen peroxide gel strip resulted in } \\
\text { greater whitening, including } 42 \% \text { to } 49 \% \text { greater improvement in tooth colour and faster whitening } \\
\text { onset than that seen with a } 6 \% \text { hydrogen peroxide whitening strip, without clinical evidence of in- } \\
\text { creased oral-tissue irritation" }\end{array}$ \\
\hline & Not able to retrieve full text \\
\hline & $\begin{array}{l}\text { Contact: Dr Robert W Gerlach, Hill Top Research, West Palm Beach, Florida, USA, gerlach.r- } \\
\text { w@pg.com }\end{array}$ \\
\hline
\end{tabular}


Guerrero 2007

\begin{tabular}{ll}
\hline Methods & $\begin{array}{l}\text { Title: clinical response of a professional whitening strip system. A randomised, double-blind, place- } \\
\text { bo-controlled study }\end{array}$ \\
\hline Participants & A total of 30 volunteer students and staff at the National Autonomous University of Mexico \\
\hline Interventions & $6.5 \%$ hydrogen peroxide strips \\
& Placebo strips \\
\hline Outcomes & Safety and efficacy of bleaching strips \\
\hline Notes & Full text not available \\
\hline
\end{tabular}

\section{Heymann 1998}

Methods

Title: clinical evaluation of 2 carbamide peroxide tooth-whitening agents

Trial design: blinded-study

Location: not available

Language: English

Number of centres: not available

Recruitment period: not available

Funding source: not available

Participants: not available
Total number: 51
Inclusion criteria: not available
Exclusion criteria: not available
Number randomised: not available
Method of randomisation: not available
Method of allocation concealment: not available
Method of blinding: not available
Number evaluated: not available

Total number of intervention groups: 2

10\% carbamide peroxide: 2 different brands

Duration of treatment: 1 week

\begin{tabular}{ll}
\hline Outcomes & Improvement in tooth shade \\
\hline Notes & Sample size calculation: not available \\
& Adverse effects: tooth sensitivity and gingival irritation \\
& Health-related quality of life: not available
\end{tabular}


Key conclusions of the study authors: "Not significant difference in the bleaching was noted between 2 groups. Mild gingival irritation and hypersensitivity was noticed between both the groups"

Not able to retrieve full text
Methods
Title: bleaching effects on colour, chemical, and mechanical properties of white spot lesions

Trial design: randomised, double-blinded, placebo-controlled trial

Location: not given in abstract

Language: English

Number of centres: 1

Recruitment period: not given in abstract

Funding source: not given in abstract

\section{Participants: not reported}

Total number: 40

Inclusion criteria: not reported in abstract

Exclusion criteria: not reported in abstract

Number randomised: 40

Method of randomisation: not reported in abstract

Method of allocation concealment: not reported in abstract

Method of blinding: not reported in abstract

Number evaluated: not reported in abstract

Total number of intervention groups: 5

5 groups: 2 test groups (strip and paint-on), 2 negative control groups and 1 positive control group (dentist-supervised home bleaching)

Duration of treatment: 4 weeks

\begin{tabular}{ll}
\hline Outcomes & Improvement in tooth shade \\
\hline Notes & Yet to procure full text for this article \\
\hline
\end{tabular}


Maran 2018 (Continued)
Language: English
Number of centres: 1
Recruitment period: not given in the abstract
Funding source: not given in the abstract

\begin{tabular}{ll}
\hline Participants & Participants: not reported \\
& Total number: 60 \\
& Inclusion criteria: not reported in abstract \\
& Exclusion criteria: not reported in abstract \\
& Number randomised: 60 \\
& Method of randomisation: not reported in abstract \\
& Method of allocation concealment: not reported in abstract \\
& Method of blinding: not reported in abstract \\
& Number evaluated: not reported in abstract \\
\hline Interventions & Total number of intervention groups: 2 \\
& Desensitizing-containing (3\% potassium nitrate and $0.2 \%$ sodium fluoride) and desensitizing-free \\
& $10 \%$ carbamide peroxide gel \\
& Duration of treatment: 21 days \\
\hline Outcomes & Improvement in tooth shade \\
\hline Yet to procure full text for this article
\end{tabular}

Number of centres: 1

Recruitment period: not reported

Funding source: Johnson \& Johnson Consumer and Personal Products Worldwide

Participants: 18 to 65 years
Total number: 225
Inclusion criteria:
- able to comprehend and follow the requirements of the study (including availability on scheduled
visits)
- able to provide written informed consent


NCT02151058 (Continued)

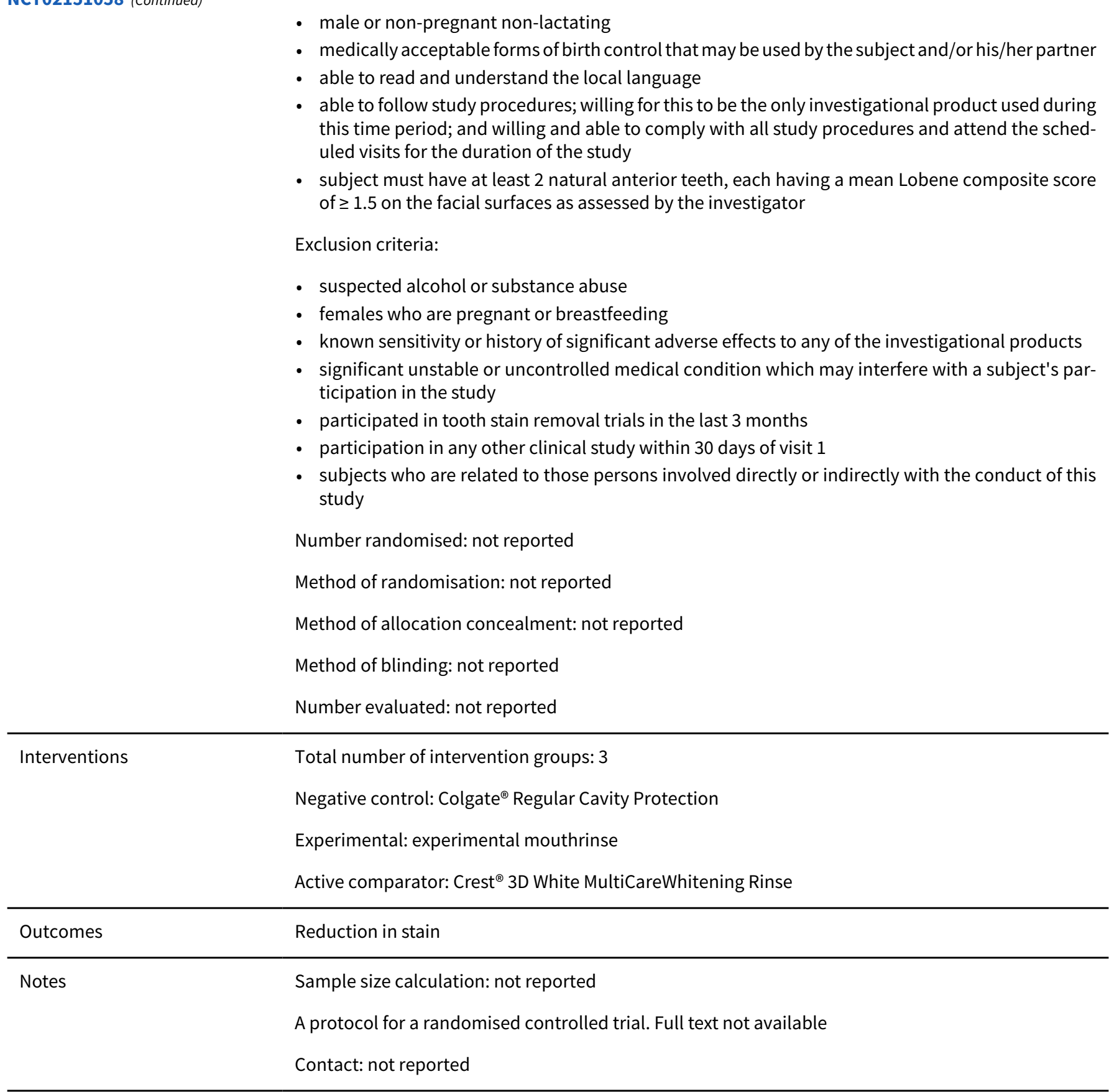

\section{NCT03217994}

\section{Methods}

Title: efficacy of 2 teeth whitening gels. A prospective, double-blind, randomised clinical trial with split-mouth design

\section{Participants}

Adults without prior tooth whitening treatments, tooth decay, or restorations of the upper front teeth. The patients had tooth colours of A3 or less according to the Vita classical scale

$6 \%$ hydrogen peroxide gel to bleach teeth 
NCT03217994 (Continued)

Outcomes Change in tooth colour

Notes A protocol for a randomised controlled trial. Full text not available

\section{Ozcan 2003}

\section{Methods}

Title: the efficacy of 2 prototype chewing gums for the removal of extrinsic tooth stain

Trial design: Double-blinded, randomised controlled trail, parallel design

Location: Marmra University, Istanbul, Turkey

Language: English

Number of centres: 1

Recruitment period: not reported

Funding source: Dandy Sakiz ve Sekerleme Sanayi ve Ticaret AS, Istanbul

Participants: 18 to 24 years. Mean age 20.6 years
Total number: 76
Inclusion criteria: healthy Individual; 12 score able anterior teeth; no orthodontic restoration
Exclusion criteria: pregnant or lactating women
Number randomised: 60
Method of randomisation: not reported
Method of allocation concealment: not reported
Method of blinding: not reported
Number evaluated: not reported

Interventions

Total number of intervention groups: 2

Group 1: sorbitol powder, maltilol syrup, mannitol, glycerine and flavour, no active ingredients

Group 2: sorbitol powder, maltilol syrup, mannitol, glycerine and flavour, sodium tripolyposphate, dicalcium phosphate and sodium bicarbonate 1:4:5

Duration of treatment: 4 weeks

\begin{tabular}{ll}
\hline Outcomes & $\begin{array}{l}\text { Reduction of stain in teeth and gingiva } \\
\text { Gingiva and body were scored by 4-point intensity scale ranging from no stain (0) to heavy stain (3) } \\
\text { and a 4-point area scale ranging from no stain (0) to stain covering greater than 2/3 of region (3) }\end{array}$ \\
\hline Sotes & Sample size calculation: not reported \\
Adverse effects: reported & Health-related quality of life: reported \\
& Key conclusions of the study authors: "The overall difference between the stain scores after 4- \\
weeks' use of the chewing gums was statistically significant for both test Product A (without active \\
ingredient) and Product B (with active ingredient) with regard to the mean baseline stain scores.
\end{tabular}


This difference represented a $48 \%$ reduction in stain scores for those subjects using Product $A$, while the reduction was $64 \%$ for the subjects using Product B"

Correspondence required: yes: missing data. Authors have been contacted

Contact: Assistant Professor Dr Mutlu Ozcan, University of Groningen, Faculty of Medical Sciences, Oral Health Institute, Antonius Deusinglaan 1, 9713 AV, Groningen, The Netherlands; mutluozcan@hotmail.com

Title: sensitivity and tooth whitening agents

Trial design: randomised controlled trial

Location: Medical College of Georgia

Language: English

Number of centres: 1

Recruitment period: not reported

Funding source: not reported

\section{Participants}

Participants: 18 years old and above

Total number: 12

Inclusion criteria: no medical condition; minimal gingival inflammation; no previous history of vital bleaching; A3 shade or above

Exclusion criteria: active caries

Number randomised: 12

Method of randomisation: not reported

Method of allocation concealment: not reported

Method of blinding: not reported

Number evaluated: not reported

Total number of intervention groups: 3

3 brands of commercial product with 10\% carbamide peroxide

Rembrandt Xtra comfort

Nite White Excel

Fx product

Duration of treatment: 2 weeks

\begin{tabular}{ll}
\hline Outcomes & Improvement in tooth shade \\
& Vita shade guide \\
\hline Notes & Sample size calculation: not reported \\
& Adverse effects: sensitivity and gingival irritation
\end{tabular}


Pohjola 2002 (Continued)

Key conclusions of the study authors: "There was no significant difference between the products with respect to improvement in tooth whitening. All 3 products produced sensitivity. Thermal sensitivity was less with Rembrandt Xtra comfort and Nite White Excel"

Correspondence required: yes: missing data, P-value not reported. Authors have been contacted

Contact: Dr Randall M Pohjola, rpobjola2mail.mcg.edu

\section{Reinhardt 1993}

Methods

Title: a clinical study of nightguard vital bleaching

Trial design: double-blinded, randomised controlled clinical trial

Location: University of lowa, USA

Language: English

Number of centres: 1

Recruitment period: not reported

Funding source: Dentmart and Omnii International

Participants

Participants: not reported

Total number: 56

Inclusion criteria: not reported

Exclusion criteria: patients with significant periodontal disease; internal tooth staining

Number randomised: 56

Method of randomisation: not reported

Method of allocation concealment: mentioned, but method not reported

Method of blinding: not reported

Number evaluated: 56

Interventions

Total number of intervention groups: 4 (3 different tray-based brands versus placebo). Intervention with 2 different application regimens for each

Proxigei

- Overnight $n=9$

- 3 hours $n=8$

White \& Brite

- Overnight $n=6$

- 3 hours $n=9$

Rembrandt Lighten

- Overnight $\mathrm{n}=8$

- 3 hours $n=8$

Control 
Reinhardt 1993 (Continued)

- Overnight $n=4$

- 3 hours $n=4$

Duration of treatment: 3 weeks

Outcomes Improvement in shade: Vita shade guide: lightest to darkest using Munsell Value

Gingival Index: 0 = no inflammation; I = inflammation, but no bleeding; 2 = bleeding on probing; and $3=$ spontaneous bleeding

Plaque Index: 0 = no plaque; $1=$ plaque detectable; 2 = plaque from interproximal surface to interproximal surface; $3=$ plaque on more than half of tooth

Notes

Sample size calculation: not reported

Adverse effects: sensitivity

Health-related quality of life: not reported

Key conclusions of the study authors: "The overnight ( 1 application) method produced toothlightening resulted at least equivalent to those of the multiple application (3-application, 3-hour) method. When Proxigei or Rembrandt was used, overnight and 3-hour replenishment produced similar results. The use of White \& Brite overnight also produced results similar to those of Rembrandt and Proxigei. The least effective treatment was White \& Brite used with 3-hour replenishment"

Correspondence required: yes: the percentage of the active ingredient used in the trial is not clear. Authors contacted

Contact: John W Reinhardt, Department of Operative Dentistry, University of lowa College of Dentistry, lowa City 52242, USA

\section{Rosenstiel 1996}

Methods

Title: randomised clinical trial of the efficacy and safety of a home bleaching procedure

Trial design: double-blinded, randomised controlled trial

Location: Ohio State University College, Columbus, Ohio, USA

Language: English

Number of centres: 1

Funding source: Ultradent

Total number: 52

Inclusion criteria: 6 maxillary anterior teeth; free of restoration/caries

Exclusion criteria: intrinsic staining; hypoplasia; fluorosis

Number randomised: 52

Method of randomisation: not reported

Method of allocation concealment: not reported

Method of blinding: not reported 


\begin{tabular}{|c|c|}
\hline \multirow[t]{4}{*}{ Interventions } & Total number of intervention groups: 2 \\
\hline & $10 \%$ carbamide peroxide \\
\hline & Placebo \\
\hline & Duration of treatment: 5 days \\
\hline \multirow[t]{4}{*}{ Outcomes } & $\begin{array}{l}\text { Colour change: } b \text { (decreased } b^{\star} \text { indicates reduced yellowness); } \Delta L^{*} \text { (increased } \Delta L^{\star} \text { is increased } \\
\text { brightness) }\end{array}$ \\
\hline & Sulcus depth \\
\hline & Vitality \\
\hline & Gingival Index \\
\hline \multirow[t]{6}{*}{ Notes } & Sample size calculation: not reported \\
\hline & Adverse effects: sensitivity and gingival irritation \\
\hline & Health-related quality of life: not reported \\
\hline & $\begin{array}{l}\text { Key conclusions of the study authors: "The control group significant reduction in mean colour } \\
\text { change at the end of } 6 \text { months. The mean colour change was more in canines compared to central } \\
\text { incisor. Lightness was significantly increased for the active canines but less with central incisors. } \\
\text { There was not change in vitality, sulcus depth or Gingival Index score" }\end{array}$ \\
\hline & $\begin{array}{l}\text { Correspondence required: yes: missing data, mean and standard deviation not reported. Authors } \\
\text { have been mailed requesting for the data. }\end{array}$ \\
\hline & $\begin{array}{l}\text { Contact: Dr Stephen F Rosenstiel, Section of Restorative and Prosthetic Dentistry, Ohio State Uni- } \\
\text { versity, College of Dentistry, } 305 \text { West 12th Avenue, Columbus, Ohio 43210, USA }\end{array}$ \\
\hline
\end{tabular}

Trial design: randomised, double-blinded, placebo-controlled trial

Location: not given in the abstract

Language: English

Number of centres: 1

Recruitment period: not given in the abstract

Funding source: not given in the abstract

Participants

Participants: not reported

Total number: 50

Inclusion criteria: not reported in abstract

Exclusion criteria: not reported in abstract

Number randomised: 50 
Rossi 2018 (Continued)

Method of randomisation: not reported in abstract

Method of allocation concealment: not reported in abstract

Method of blinding: not reported in abstract

Number evaluated: not reported in abstract

Interventions

Total number of intervention groups: 2

$10 \%$ hydrogen peroxide strip versus tray

Placebo

Duration of treatment: 14 days

Outcomes Improvement in tooth shade

Notes Yet to procure full text for this article

Shin 2010

Title: the evaluation of clinical efficacy and longevity of home bleaching without combined applica-
tion of in-office bleaching
Trial design: randomised controlled trial
Location: not reported
Language: Korean
Number of centres: 1
Recruitment period: not reported
Funding source: not reported

Participants

Participants: age 19 to 40 years

Total number: 28

Inclusion criteria: mild tooth discolouration; 6 anterior teeth present

Exclusion criteria: resin filling; porcelain restoration; dental caries; gingivitis and periodontitis

Number randomised: not reported

Method of randomisation: not reported

Method of allocation concealment: not reported

Method of blinding: not reported

Number evaluated: not reported

Interventions

Total number of intervention groups: 2

$15 \%$ carbamide peroxide tray

Placebo

Duration of treatment: 4 weeks 
Shin 2010 (Continued)

Outcomes Change in tooth colour: $b$ (decreased $b^{*}$ indicates reduced yellowness); $\Delta \mathrm{L}^{*}$ (increased $\Delta \mathrm{L}^{*}$ is increased brightness)

Notes

Sample size calculation: not reported

Adverse effects: not reported

Health-related quality of life: not reported

Key conclusions of the study authors: "Stronger colour change was observed for overall teeth samples in experimental group immediately after treatment (at 4 weeks) compared to ones in control group. There was also a significant difference between baseline and 8 weeks or 12 weeks as the tooth got darker with time)

Correspondence required: yes: missing data, number of participants in control and experimental groups not reported. Authors have been mailed requesting for the data

Contact: Sung Wun Yang, dentyun@catholic.ac.kr

\section{Sielski 2003}

\begin{tabular}{ll}
\hline Methods & $\begin{array}{l}\text { Randomised, controlled, examiner-blinded, parallel-group clinical study to determine efficacy of } \\
\text { tooth-whitening gel when used once daily at night, as compared with a commercially available } \\
\text { dentifrice }\end{array}$ \\
\hline Participants & 75 adults \\
\hline Interventions & Non-whitening dentifrice only \\
& A tooth-whitening gel with a commercially available dentifrice \\
\hline Outcomes & Change in tooth colour \\
\hline Notes & Full text not available \\
\hline
\end{tabular}

\section{Simon 2014}

Methods

Title: placebo-controlled clinical trial evaluating $9.5 \%$ hydrogen peroxide high-adhesion whitening strips

Trial design: parallel, double-blinded, randomised controlled trial

Location: University of Tennessee, USA

Language: English

Number of centres: 1

Funding source: Procter \& Gamble

Participants: 18 to 65 years
Total number: 54
Inclusion criteria: 4 teeth which are A2 shade or darker; healthy adults
Exclusion criteria: intrinsic staining; tooth sensitivity; orthodontic device


Simon 2014 (Continued)

Number randomised: not mentioned

Method of randomisation: not mentioned

Method of allocation concealment: not mention

Method of blinding: not mentioned

Number evaluated: 54

\begin{tabular}{ll}
\hline Interventions & Total number of intervention groups: 2 \\
& $9.5 \%$ hydrogen peroxide strips \\
& Placebo \\
& Duration of treatment: 3 weeks \\
\hline Outcomes & Tooth colour change: $b$ (decreased $b^{\star}$ indicates reduced yellowness); $\Delta \mathrm{L}^{\star}$ (increased $\Delta \mathrm{L}^{\star}$ is in- \\
creased brightness) & Tooth sensitivity \\
\hline Notes & Sample size calculation: not reported \\
& Adverse effects: oral irritation and tooth sensitivity \\
& Health-related quality of life: not reported \\
& Key conclusions of the study authors: "Experimental 9.5\% hydrogen peroxide strip yielded signifi- \\
cant tooth whitening relative to a placebo strip as early as after three days of product use" & Correspondence required: yes: authors have been contacted for missing data \\
Contact: Dr James $F$ Simon, farrells.2@pg.com
\end{tabular}

Characteristics of ongoing studies [ordered by study ID]

NCT03026725

Trial name or title

Effect of tricalcium phosphate on efficacy and sensitivity with vital tooth whitening using $20 \%$ carbamide peroxide

\begin{tabular}{ll}
\hline Methods & Randomised controlled trial \\
\hline Participants & Not given \\
\hline Interventions & Not given \\
\hline Outcomes & Improvement in shade \\
\hline Starting date & 2017 \\
\hline Contact information & clinicaltrials.gov/show/nct03026725 \\
\hline Notes & Results not yet reported \\
\hline
\end{tabular}




\section{DATA AND ANALYSES}

\section{Comparison 1. CP gel in tray versus placebo}

\begin{tabular}{|c|c|c|c|c|}
\hline Outcome or subgroup title & No. of studies & $\begin{array}{l}\text { No. of partici- } \\
\text { pants }\end{array}$ & Statistical method & Effect size \\
\hline $\begin{array}{l}1 \text { Tooth whitening - assessed by the } \\
\text { dentist }\end{array}$ & 3 & & $\begin{array}{l}\text { Mean Difference (IV, Fixed, 95\% } \\
\mathrm{CI})\end{array}$ & Subtotals only \\
\hline $1.15 \%$ CP gel - 2 weeks & 1 & 21 & $\begin{array}{l}\text { Mean Difference (IV, Fixed, 95\% } \\
\mathrm{CI})\end{array}$ & $4.56[1.52,7.59]$ \\
\hline $\begin{array}{l}1.210 \% \text { CP gel with desensitiser - } 2 \\
\text { weeks }\end{array}$ & 1 & 37 & $\begin{array}{l}\text { Mean Difference (IV, Fixed, 95\% } \\
\mathrm{Cl} \text { ) }\end{array}$ & $4.70[3.28,6.12]$ \\
\hline $\begin{array}{l}1.310 \% \text { CP gel - light shade - } 2 \\
\text { weeks }\end{array}$ & 1 & 179 & $\begin{array}{l}\text { Mean Difference (IV, Fixed, 95\% } \\
\mathrm{Cl})\end{array}$ & $4.5[4.04,4.96]$ \\
\hline $\begin{array}{l}1.410 \% \text { CP gel - medium dark shade } \\
\text { - } 2 \text { weeks }\end{array}$ & 1 & 172 & $\begin{array}{l}\text { Mean Difference (IV, Fixed, 95\% } \\
\mathrm{Cl})\end{array}$ & $6.90[6.35,7.45]$ \\
\hline $\begin{array}{l}1.510 \% \text { CP gel - darker shade - } 2 \\
\text { weeks }\end{array}$ & 1 & 176 & $\begin{array}{l}\text { Mean Difference (IV, Fixed, 95\% } \\
\mathrm{Cl} \text { ) }\end{array}$ & $10.0[9.44,10.56]$ \\
\hline
\end{tabular}

\section{Analysis 1.1. Comparison 1 CP gel in tray versus placebo, Outcome 1 Tooth whitening - assessed by the dentist.}

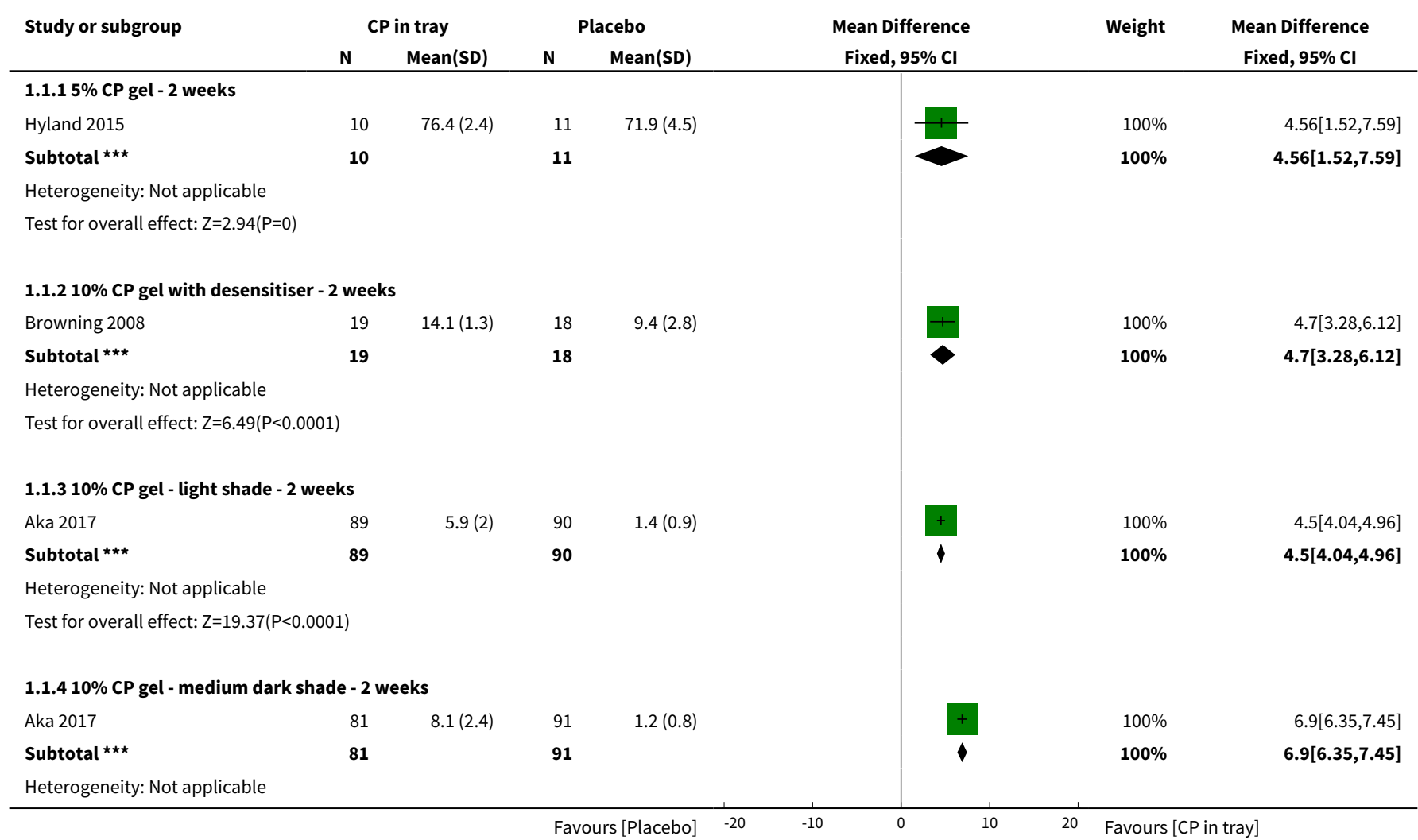




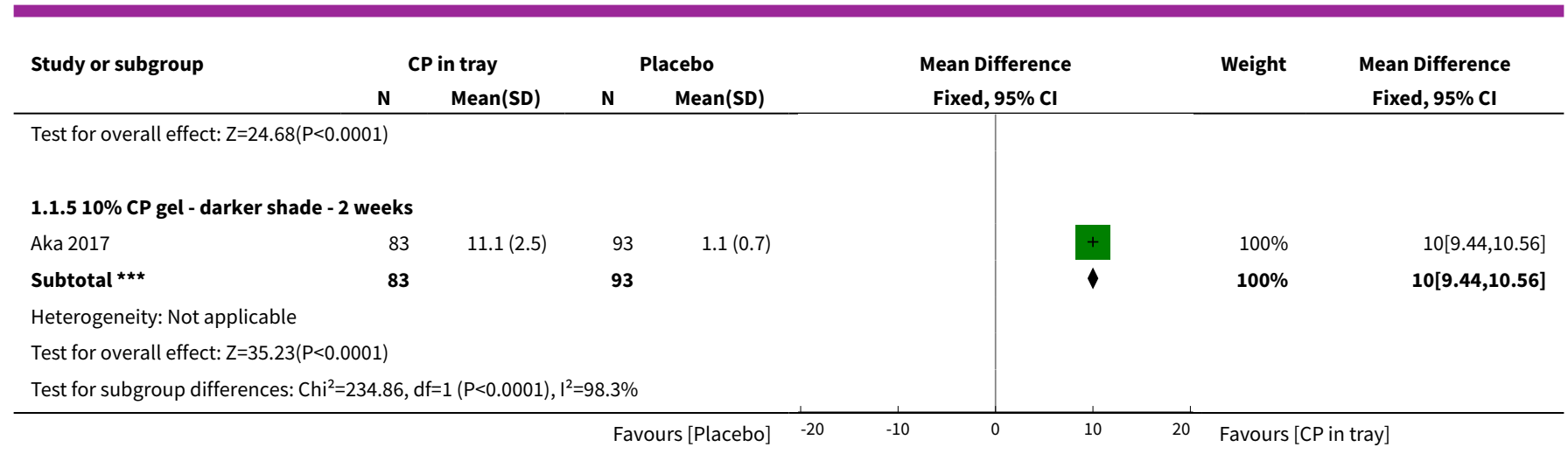

\section{Comparison 2. CP gel in tray versus placebo}

\begin{tabular}{lllll}
\hline Outcome or subgroup title & No. of studies & $\begin{array}{l}\text { No. of partici- } \\
\text { pants }\end{array}$ & Statistical method & Effect size \\
\hline $\begin{array}{l}1 \text { Tooth whitening - assessed by the } \\
\text { dentist }\end{array}$ & 2 & 109 & $\begin{array}{l}\text { Risk Ratio (M-H, Random, 95\% } \\
\text { Cl) }\end{array}$ & $6.74[3.15,14.40]$ \\
\hline $1.110 \%$ CP gel - 6 months & 2 & 109 & $\begin{array}{l}\text { Risk Ratio (M-H, Random, 95\% } \\
\text { Cl) }\end{array}$ & $6.74[3.15,14.40]$ \\
\hline
\end{tabular}

\section{Analysis 2.1. Comparison 2 CP gel in tray versus placebo, Outcome 1 Tooth whitening - assessed by the dentist.}

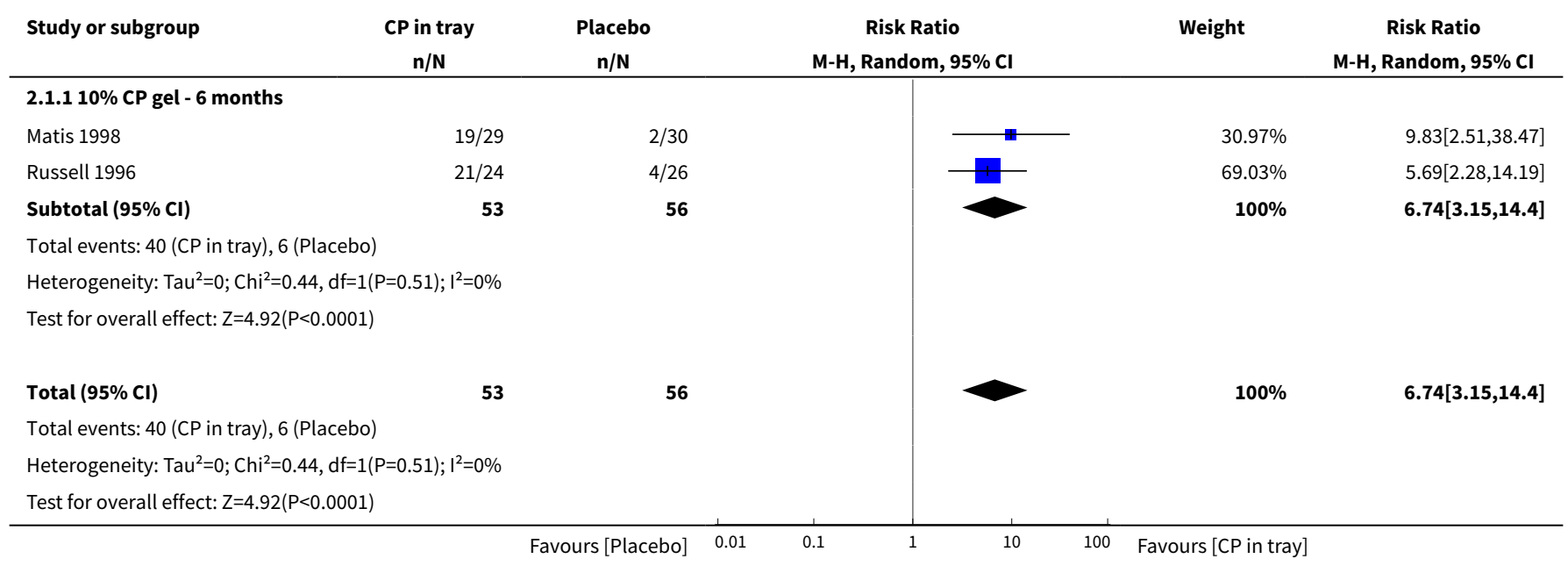

\section{Comparison 3. HP gel in tray versus placebo}

\begin{tabular}{lllll}
\hline Outcome or subgroup title & No. of studies & $\begin{array}{l}\text { No. of partici- } \\
\text { pants }\end{array}$ & Statistical method & Effect size \\
\hline $\begin{array}{l}1 \text { Tooth whitening - assessed by } \\
\text { the dentist }\end{array}$ & 1 & $\begin{array}{l}\text { Mean Difference (IV, Random, 95\% } \\
\text { Cl) }\end{array}$ & Subtotals only \\
\hline
\end{tabular}




\begin{tabular}{lllll}
\hline Outcome or subgroup title & No. of studies & $\begin{array}{l}\text { No. of partici- } \\
\text { pants }\end{array}$ & Statistical method & Effect size \\
\hline $1.16 \%$ HP gel - 14 days & 1 & 49 & $\begin{array}{l}\text { Mean Difference (IV, Random, 95\% } \\
\text { Cl) }\end{array}$ & $3.08[2.28,3.88]$ \\
\hline
\end{tabular}

Analysis 3.1. Comparison 3 HP gel in tray versus placebo, Outcome 1 Tooth whitening - assessed by the dentist.

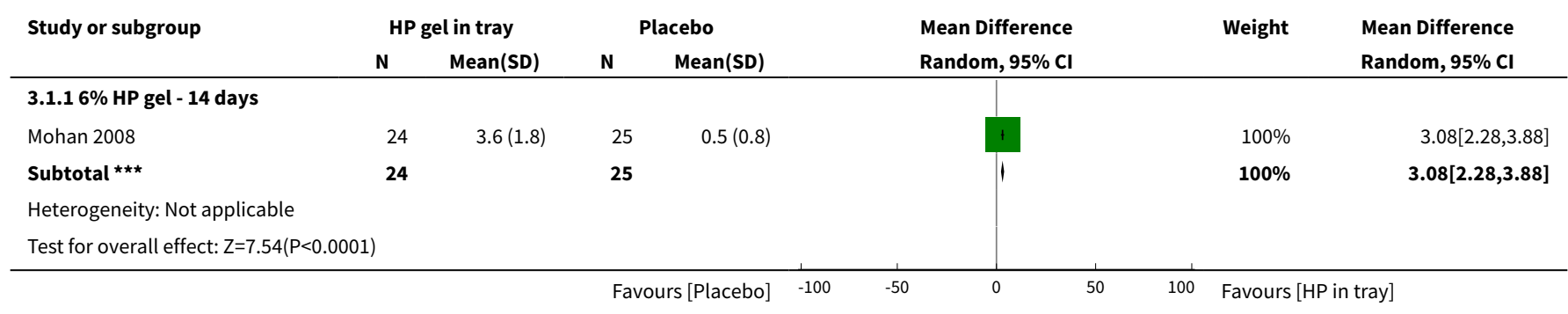

\section{Comparison 4. HP strip versus placebo}

\begin{tabular}{lllll}
\hline $\begin{array}{l}\text { Outcome or subgroup ti- } \\
\text { tle }\end{array}$ & No. of studies & $\begin{array}{l}\text { No. of partici- } \\
\text { pants }\end{array}$ & Statistical method & Effect size \\
\hline $\begin{array}{l}1 \text { Tooth whitening - as- } \\
\text { sessed by the dentist }\end{array}$ & 9 & & Mean Difference (IV, Random, 95\% Cl) & Subtotals only \\
\hline $1.110 \% \mathrm{HP}$ - day 8 & 1 & 36 & Mean Difference (IV, Random, 95\% Cl) & $2.24[1.72,2.76]$ \\
\hline $1.26 \% \mathrm{HP}-2$ weeks & 4 & 195 & Mean Difference (IV, Random, 95\% Cl) & $2.24[1.83,2.66]$ \\
\hline $1.310 \% \mathrm{HP}-15$ days & 1 & 40 & Mean Difference (IV, Random, 95\% Cl) & $1.93[1.34,2.52]$ \\
\hline $1.414 \% \mathrm{HP}-3$ weeks & 1 & 28 & Mean Difference (IV, Random, 95\% Cl) & $2.90[1.73,4.07]$ \\
\hline $1.56 \% \mathrm{HP}-6$ weeks & 1 & 37 & Mean Difference (IV, Random, 95\% Cl) & $5.16[4.21,6.11]$ \\
\hline $1.614 \% \mathrm{HP}-6$ weeks & 1 & 35 & Mean Difference (IV, Random, 95\% Cl) & $1.21[0.67,1.75]$ \\
\hline $1.75 .3 \%$ HP - 6 months & 1 & 52 & & $7.6[6.18,9.02]$ \\
\hline
\end{tabular}

Analysis 4.1. Comparison 4 HP strip versus placebo, Outcome 1 Tooth whitening - assessed by the dentist.

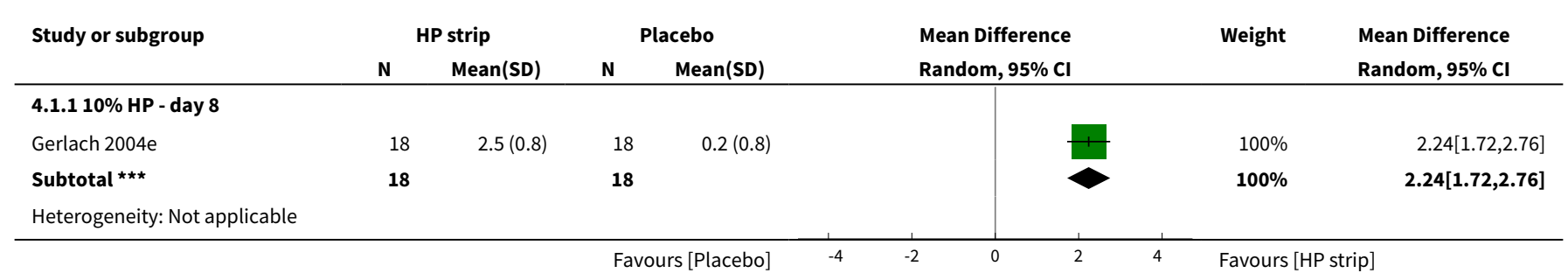




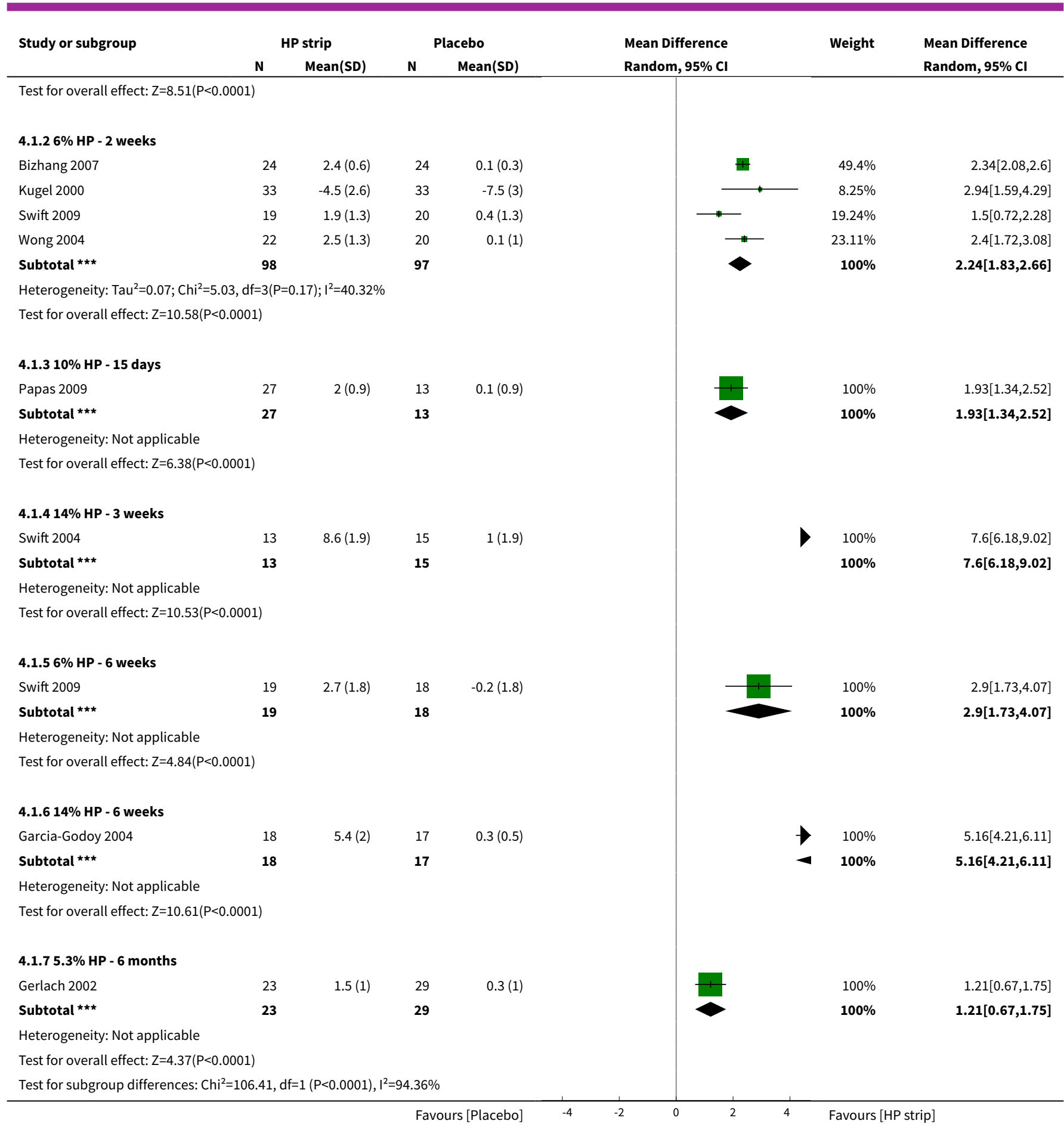

\section{Comparison 5. CP paint-on gel versus placebo}

\begin{tabular}{lllll}
\hline Outcome or subgroup title & No. of studies & $\begin{array}{l}\text { No. of partici- } \\
\text { pants }\end{array}$ & Statistical method & Effect size \\
\hline $\begin{array}{l}1 \text { Tooth whitening - assessed by the } \\
\text { dentist }\end{array}$ & 1 & & $\begin{array}{l}\text { Mean Difference (IV, Random, } \\
95 \% \mathrm{Cl})\end{array}$ & Subtotals only \\
\hline
\end{tabular}




\begin{tabular}{lllll}
\hline Outcome or subgroup title & No. of studies & $\begin{array}{l}\text { No. of partici- } \\
\text { pants }\end{array}$ & Statistical method & Effect size \\
\hline $1.118 \%$ CP paint-on gel - 3 weeks & 1 & 77 & $\begin{array}{l}\text { Mean Difference (IV, Random, } \\
95 \% \text { Cl) }\end{array}$ & $3.50[3.12,3.88]$ \\
\hline
\end{tabular}

Analysis 5.1. Comparison $5 \mathrm{CP}$ paint-on gel versus placebo, Outcome 1 Tooth whitening - assessed by the dentist.

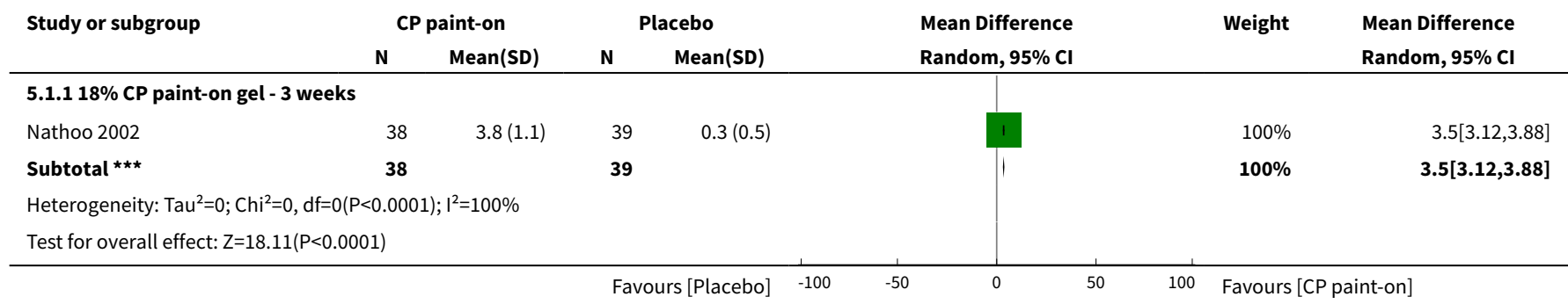

\section{Comparison 6. HP paint-on gel versus placebo}

\begin{tabular}{lllll}
\hline Outcome or subgroup title & No. of studies & $\begin{array}{l}\text { No. of partici- } \\
\text { pants }\end{array}$ & Statistical method & Effect size \\
\hline $\begin{array}{l}1 \text { Tooth whitening - assessed by } \\
\text { the dentist }\end{array}$ & 2 & & $\begin{array}{l}\text { Std. Mean Difference (IV, Random, } \\
95 \% \mathrm{Cl})\end{array}$ & Subtotals only \\
\hline $1.16 \% \mathrm{HP}$ - 2 weeks & 2 & 148 & $\begin{array}{l}\text { Std. Mean Difference (IV, Random, } \\
95 \% \mathrm{Cl})\end{array}$ & $0.67[0.19,1.14]$ \\
\hline
\end{tabular}

Analysis 6.1. Comparison 6 HP paint-on gel versus placebo, Outcome 1 Tooth whitening - assessed by the dentist.

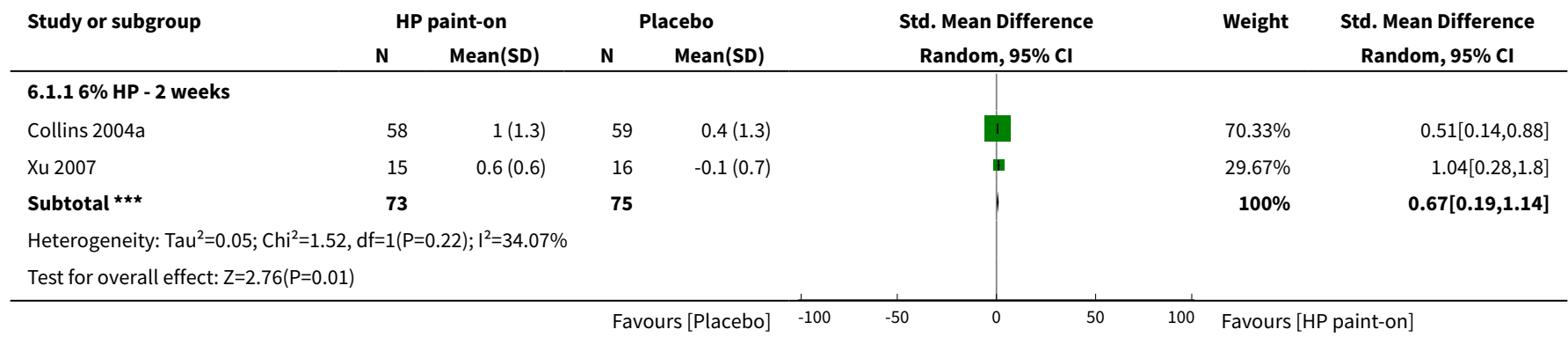


Comparison 7. Chewing gum SHMP versus placebo

\begin{tabular}{lllll}
\hline Outcome or subgroup title & No. of studies & $\begin{array}{l}\text { No. of partici- } \\
\text { pants }\end{array}$ & Statistical method & Effect size \\
\hline $\begin{array}{l}1 \text { Tooth whitening - assessed by } \\
\text { the dentist }\end{array}$ & 3 & & $\begin{array}{l}\text { Mean Difference (Random, 95\% } \\
\text { Cl) }\end{array}$ & Subtotals only \\
\hline $\begin{array}{lllll}1.17 .5 \% \text { SHMP gum - 2 days } \\
1.25 .6 \% \text { SHMP gum - } 3 \text { days }\end{array}$ & 1 & 37 & $\begin{array}{l}\text { Mean Difference (Random, 95\% } \\
\text { Cl) }\end{array}$ & $0.89[0.77,1.01]$ \\
\hline $1.34 \%$ SHMP gum - 12 weeks & 1 & 20 & $\begin{array}{l}\text { Mean Difference (Random, 95\% } \\
\text { Cl) }\end{array}$ & $2.6[1.45,3.75]$ \\
\hline
\end{tabular}

Analysis 7.1. Comparison 7 Chewing gum SHMP versus placebo, Outcome 1 Tooth whitening - assessed by the dentist.

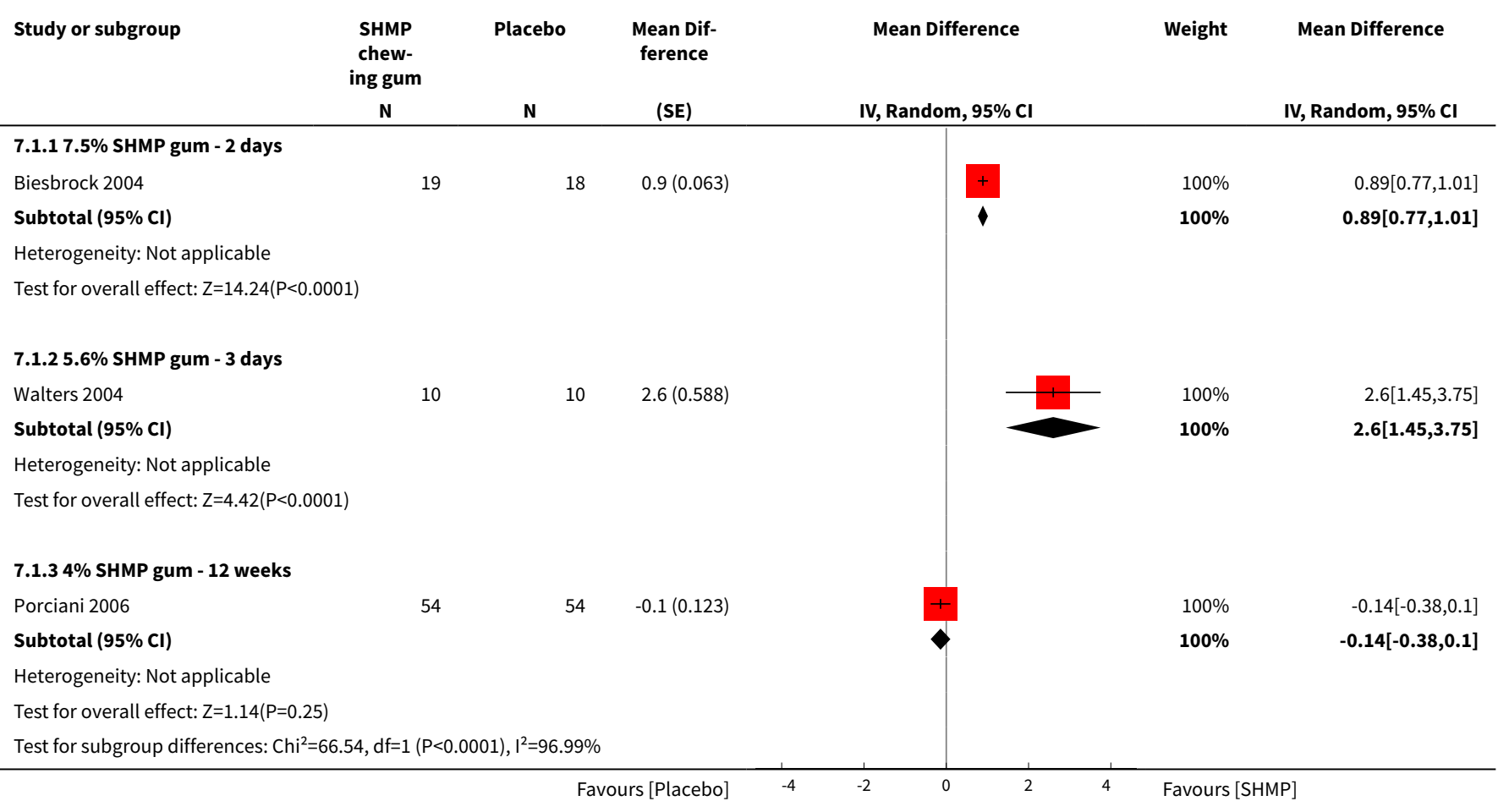

\section{Comparison 8. Chewing gum STPP versus placebo}

\begin{tabular}{lllll}
\hline Outcome or subgroup title & No. of studies & $\begin{array}{l}\text { No. of partici- } \\
\text { pants }\end{array}$ & Statistical method & Effect size \\
\hline $\begin{array}{l}1 \text { Tooth whitening - assessed by the } \\
\text { dentist }\end{array}$ & 1 & $\begin{array}{l}\text { Mean Difference (IV, Random, } \\
95 \% \mathrm{Cl})\end{array}$ & Subtotals only \\
\hline
\end{tabular}




\begin{tabular}{lllll}
\hline Outcome or subgroup title & No. of studies & $\begin{array}{l}\text { No. of partici- } \\
\text { pants }\end{array}$ & Statistical method & Effect size \\
\hline $1.11 \%$ STPP gum - 6 weeks & 1 & 108 & $\begin{array}{l}\text { Mean Difference (IV, Random, } \\
95 \% \text { CI) }\end{array}$ & $0.18[0.10,0.26]$ \\
\hline
\end{tabular}

Analysis 8.1. Comparison 8 Chewing gum STPP versus placebo, Outcome 1 Tooth whitening - assessed by the dentist.

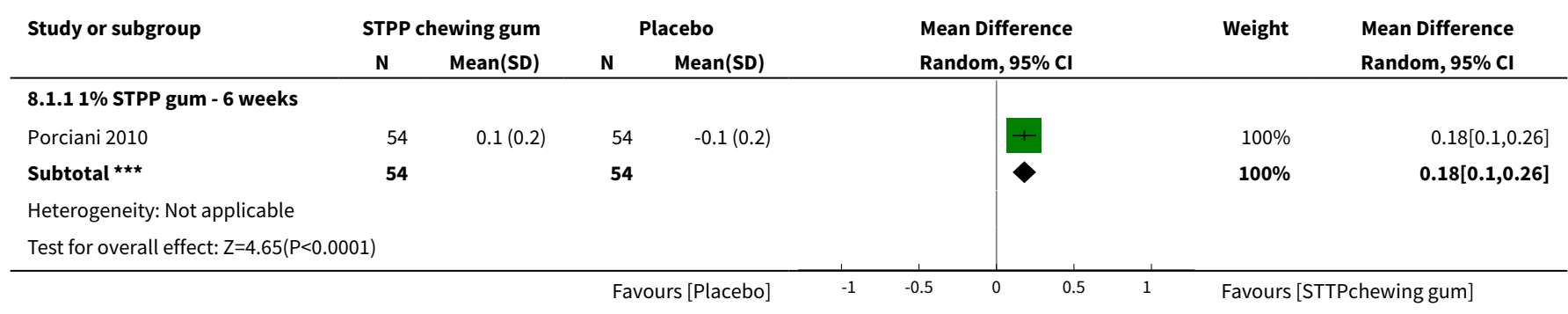

\section{Comparison 9. HP mouthwash versus placebo}

\begin{tabular}{llllll}
\hline Outcome or subgroup title & No. of studies & $\begin{array}{l}\text { No. of partici- } \\
\text { pants }\end{array}$ & Statistical method & Effect size \\
\hline 1 Tooth whitening - assessed by the dentist & 1 & & (Random, 95\% Cl) & Subtotals only \\
\hline $1.11 .5 \%$ HP + 0.05\% F - 6 months & 1 & 78 & (Random, 95\% Cl) & $10.89[5.08,23.35]$ \\
\hline
\end{tabular}

Analysis 9.1. Comparison 9 HP mouthwash versus placebo, Outcome 1 Tooth whitening - assessed by the dentist.

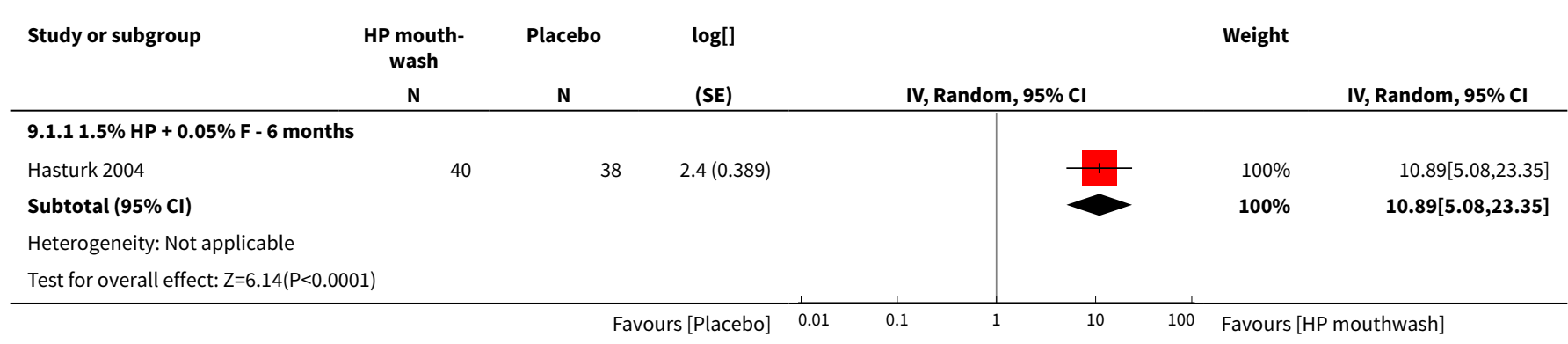

\section{Comparison 10. CP tray versus CP tray}

\begin{tabular}{lllll}
\hline Outcome or subgroup title & No. of studies & $\begin{array}{l}\text { No. of partici- } \\
\text { pants }\end{array}$ & Statistical method & Effect size \\
\hline $\begin{array}{l}1 \text { Tooth whitening - assessed by the } \\
\text { dentist }\end{array}$ & 1 & & $\begin{array}{l}\text { Risk Ratio (M-H, Random, } \\
95 \% \mathrm{Cl})\end{array}$ & Subtotals only \\
\hline
\end{tabular}




\begin{tabular}{lllll}
\hline Outcome or subgroup title & No. of studies & $\begin{array}{l}\text { No. of partici- } \\
\text { pants }\end{array}$ & Statistical method & Effect size \\
\hline $\begin{array}{l}1.110 \% \text { CP tray versus 10\% CP tray - } 2 \\
\text { weeks }\end{array}$ & 1 & 66 & $\begin{array}{l}\text { Risk Ratio (M-H, Random, } \\
95 \% \mathrm{Cl})\end{array}$ & $1.03[0.90,1.18]$ \\
\hline
\end{tabular}

Analysis 10.1. Comparison 10 CP tray versus CP tray, Outcome 1 Tooth whitening - assessed by the dentist.

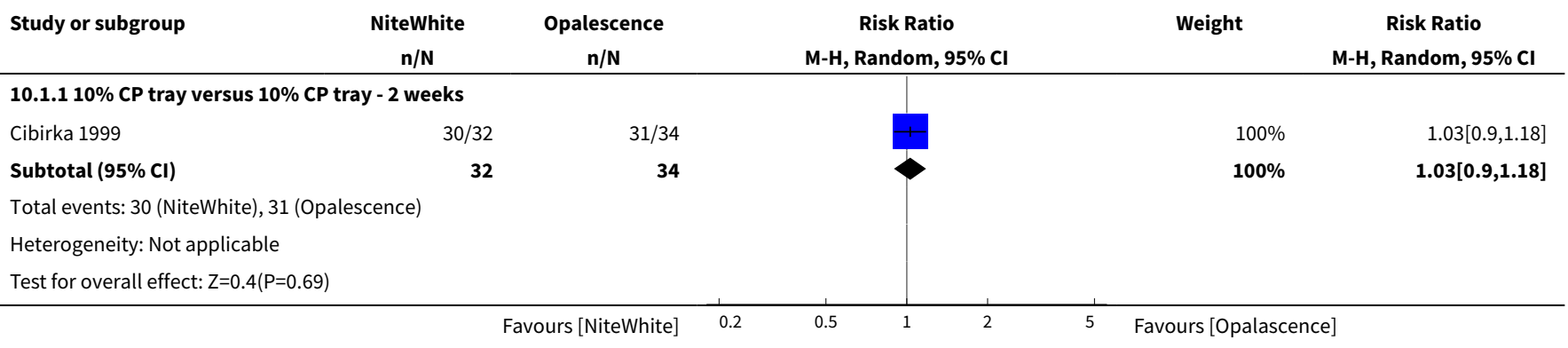

\section{Comparison 11. CP tray versus $\mathrm{CP}$ tray}

\begin{tabular}{|c|c|c|c|c|}
\hline Outcome or subgroup title & No. of studies & $\begin{array}{l}\text { No. of partici- } \\
\text { pants }\end{array}$ & Statistical method & Effect size \\
\hline $\begin{array}{l}1 \text { Tooth whitening - assessed by the } \\
\text { dentist }\end{array}$ & 9 & & $\begin{array}{l}\text { Mean Difference (IV, Random, } \\
95 \% \mathrm{CI} \text { ) }\end{array}$ & Subtotals only \\
\hline $1.15 \% \mathrm{CP}$ versus $10 \% \mathrm{CP}$ - 1 week & 1 & 58 & $\begin{array}{l}\text { Mean Difference (IV, Random, } \\
95 \% \mathrm{CI} \text { ) }\end{array}$ & $-0.38[-5.55,4.79]$ \\
\hline $1.25 \% \mathrm{CP}$ versus $10 \% \mathrm{CP}$ - 2 weeks & 1 & 21 & $\begin{array}{l}\text { Mean Difference (IV, Random, } \\
95 \% \mathrm{CI} \text { ) }\end{array}$ & $0.41[-2.17,2.98]$ \\
\hline $\begin{array}{l}1.310 \% \text { CP Colgate Platinum versus } \\
10 \% \text { CP Rembrandt Lighten - } 2 \text { weeks }\end{array}$ & 2 & 88 & $\begin{array}{l}\text { Mean Difference (IV, Random, } \\
95 \% \mathrm{CI} \text { ) }\end{array}$ & $-1.92[-2.80,-1.03]$ \\
\hline $\begin{array}{l}1.410 \% \text { CP versus } 28 \% \text { CP - } 1 \text { year fol- } \\
\text { low-up }\end{array}$ & 1 & 20 & $\begin{array}{l}\text { Mean Difference (IV, Random, } \\
95 \% \mathrm{CI} \text { ) }\end{array}$ & $-3.30[-8.71,2.11]$ \\
\hline $\begin{array}{l}1.510 \% \text { CP versus } 16 \% \text { CP - 2-year } \\
\text { follow-up }\end{array}$ & 1 & 81 & $\begin{array}{l}\text { Mean Difference (IV, Random, } \\
95 \% \mathrm{CI} \text { ) }\end{array}$ & $1.20[-0.35,2.75]$ \\
\hline $\begin{array}{l}1.630 \% \mathrm{CP} \text { versus } 30 \% \mathrm{CP}+\mathrm{KN}-10 \\
\text { days }\end{array}$ & 1 & 40 & $\begin{array}{l}\text { Mean Difference (IV, Random, } \\
95 \% \mathrm{CI} \text { ) }\end{array}$ & $-0.30[-8.28,7.68]$ \\
\hline $\begin{array}{l}1.716 \% \mathrm{CP} \text { versus } 16 \% \mathrm{CP}+\mathrm{KN}+\mathrm{NaF} \\
-4 \text { weeks }\end{array}$ & 1 & 60 & $\begin{array}{l}\text { Mean Difference (IV, Random, } \\
95 \% \mathrm{CI} \text { ) }\end{array}$ & $-0.20[-0.44,0.04]$ \\
\hline $\begin{array}{l}1.816 \% \text { CP versus } 16 \% C P+A C P-6 \\
\text { months }\end{array}$ & 1 & 27 & $\begin{array}{l}\text { Mean Difference (IV, Random, } \\
95 \% \mathrm{CI} \text { ) }\end{array}$ & $0.78[0.37,1.19]$ \\
\hline $\begin{array}{l}2 \text { Tooth whitening - assessed by the } \\
\text { dentist }\end{array}$ & 2 & & $\begin{array}{l}\text { Mean Difference (Fixed, 95\% } \\
\mathrm{Cl} \text { ) }\end{array}$ & Subtotals only \\
\hline
\end{tabular}




\begin{tabular}{lllll}
\hline Outcome or subgroup title & No. of studies & $\begin{array}{l}\text { No. of partici- } \\
\text { pants }\end{array}$ & Statistical method & Effect size \\
\hline $\begin{array}{l}2.110 \% \text { CP Polanight versus 10\% CP } \\
\text { Opalescence - 2 weeks }\end{array}$ & 1 & 116 & $\begin{array}{l}\text { Mean Difference (Fixed, 95\% } \\
\text { CI) }\end{array}$ & 1.46 [0.13, 2.79] \\
\hline $\begin{array}{l}2.210 \% \text { CP versus 15\% CP tetracy- } \\
\text { cline-stained teeth - 5 years }\end{array}$ & 1 & 58 & $\begin{array}{l}\text { Mean Difference (Fixed, 95\% } \\
\text { CI) }\end{array}$ & -1.47 [-3.56, 0.62] \\
\hline $\begin{array}{l}\text { 3 Tooth whitening - reported by the } \\
\text { patient }\end{array}$ & 1 & & $\begin{array}{l}\text { Mean Difference (IV, Fixed, } \\
95 \% \text { Cl) }\end{array}$ & Subtotals only \\
\hline $\begin{array}{l}\text { 3.1 10\% CP versus 17\% CP - Pa- } \\
\text { tient-contentment - 3 weeks }\end{array}$ & 1 & 20 & $\begin{array}{l}\text { Mean Difference (IV, Fixed, } \\
95 \% \text { CI) }\end{array}$ & 2.60 [2.57, 2.63] \\
\hline
\end{tabular}

Analysis 11.1. Comparison 11 CP tray versus CP tray, Outcome 1 Tooth whitening - assessed by the dentist.

\begin{tabular}{|c|c|c|c|c|c|c|c|}
\hline \multirow[t]{2}{*}{ Study or subgroup } & \multicolumn{2}{|c|}{ Group 1 tray } & \multicolumn{2}{|c|}{ Group 2 tray } & \multirow{2}{*}{$\begin{array}{l}\text { Mean Difference } \\
\text { Random, } 95 \% \mathrm{Cl}\end{array}$} & \multirow[t]{2}{*}{ Weight } & \multirow{2}{*}{$\begin{array}{l}\text { Mean Difference } \\
\text { Random, } 95 \% \mathrm{Cl}\end{array}$} \\
\hline & $\mathbf{N}$ & $\operatorname{Mean}(S D)$ & $\mathbf{N}$ & $\operatorname{Mean}(\mathrm{SD})$ & & & \\
\hline \multicolumn{8}{|c|}{ 11.1.1 5\% CP versus $10 \%$ CP - 1 week } \\
\hline Nathoo 2001 & 29 & $-4.4(10.2)$ & 29 & $-4(9.9)$ & & $100 \%$ & $-0.38[-5.55,4.79]$ \\
\hline Subtotal $\star \star \star$ & 29 & & 29 & & & $100 \%$ & $-0.38[-5.55,4.79]$ \\
\hline \multicolumn{8}{|c|}{ Heterogeneity: Not applicable } \\
\hline \multicolumn{8}{|c|}{ Test for overall effect: $Z=0.14(P=0.89)$} \\
\hline \multicolumn{8}{|c|}{ 11.1.2 $5 \% \mathrm{CP}$ versus $10 \% \mathrm{CP}$ - 2 weeks } \\
\hline Hyland 2015 & 10 & $-76.4(2.4)$ & 11 & $-76.8(3.5)$ & & $100 \%$ & $0.41[-2.17,2.98]$ \\
\hline 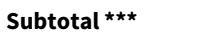 & 10 & & 11 & & & $100 \%$ & $0.41[-2.17,2.98]$ \\
\hline \multicolumn{8}{|c|}{ Heterogeneity: Not applicable } \\
\hline \multicolumn{8}{|c|}{ Test for overall effect: $\mathrm{Z}=0.31(\mathrm{P}=0.76)$} \\
\hline Kowitz 1994 & 24 & $-3.4(1.5)$ & 24 & $-1.7(1.3)$ & - & $75.5 \%$ & $-1.66[-2.44,-0.88]$ \\
\hline Nathoo 1994 & 20 & $-3.8(3.1)$ & 20 & $-1.1(2.3)$ & & $24.5 \%$ & $-2.71[-4.37,-1.05]$ \\
\hline Subtotal $* \star \star$ & 44 & & 44 & & & $100 \%$ & $-1.92[-2.8,-1.03]$ \\
\hline \multicolumn{8}{|c|}{ Heterogeneity: $\mathrm{Tau}^{2}=0.11 ; \mathrm{Chi}^{2}=1.25, \mathrm{df}=1(\mathrm{P}=0.26) ; \mathrm{I}^{2}=20.3 \%$} \\
\hline \multicolumn{8}{|c|}{ Test for overall effect: $Z=4.25(P<0.0001)$} \\
\hline \multicolumn{8}{|c|}{ 11.1.4 10\% CP versus $28 \%$ CP - 1 year follow-up } \\
\hline Turkun 2010 & 10 & $-80.9(6.6)$ & 10 & $-77.6(5.7)$ & & $100 \%$ & $-3.3[-8.71,2.11]$ \\
\hline Subtotal $* \star \star$ & 10 & & 10 & & & $100 \%$ & $-3.3[-8.71,2.11]$ \\
\hline \multicolumn{8}{|c|}{ Heterogeneity: Not applicable } \\
\hline \multicolumn{8}{|c|}{ Test for overall effect: $Z=1.2(P=0.23)$} \\
\hline \multicolumn{8}{|c|}{ 11.1.5 10\% CP versus $16 \%$ CP - 2-year follow-up } \\
\hline Subtotal $\star \star \star$ & 42 & & 39 & & & $100 \%$ & $1.2[-0.35,2.75]$ \\
\hline \multicolumn{8}{|c|}{ Heterogeneity: Not applicable } \\
\hline \multicolumn{8}{|c|}{ Test for overall effect: $Z=1.52(P=0.13)$} \\
\hline \multicolumn{8}{|c|}{$11.1 .630 \% \mathrm{CP}$ versus $30 \% \mathrm{CP}+\mathrm{KN}-10$ days } \\
\hline Gallo 2009 & 20 & $17(6.5)$ & 20 & $17.3(17)$ & & $100 \%$ & $-0.3[-8.28,7.68]$ \\
\hline & & & ours & oup 1 tray] & -2.5 & Favours & tray] \\
\hline
\end{tabular}




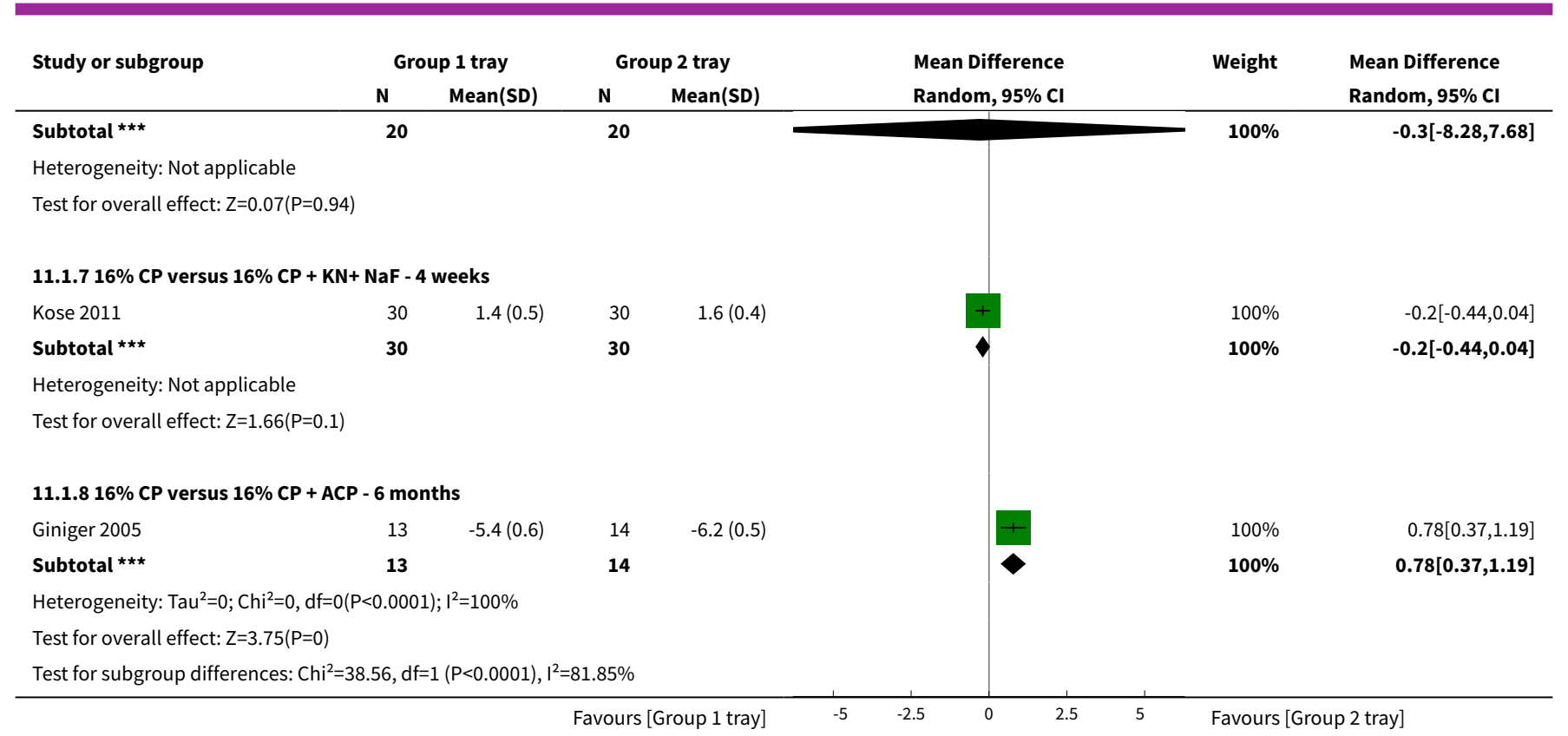

Analysis 11.2. Comparison 11 CP tray versus CP tray, Outcome 2 Tooth whitening - assessed by the dentist.

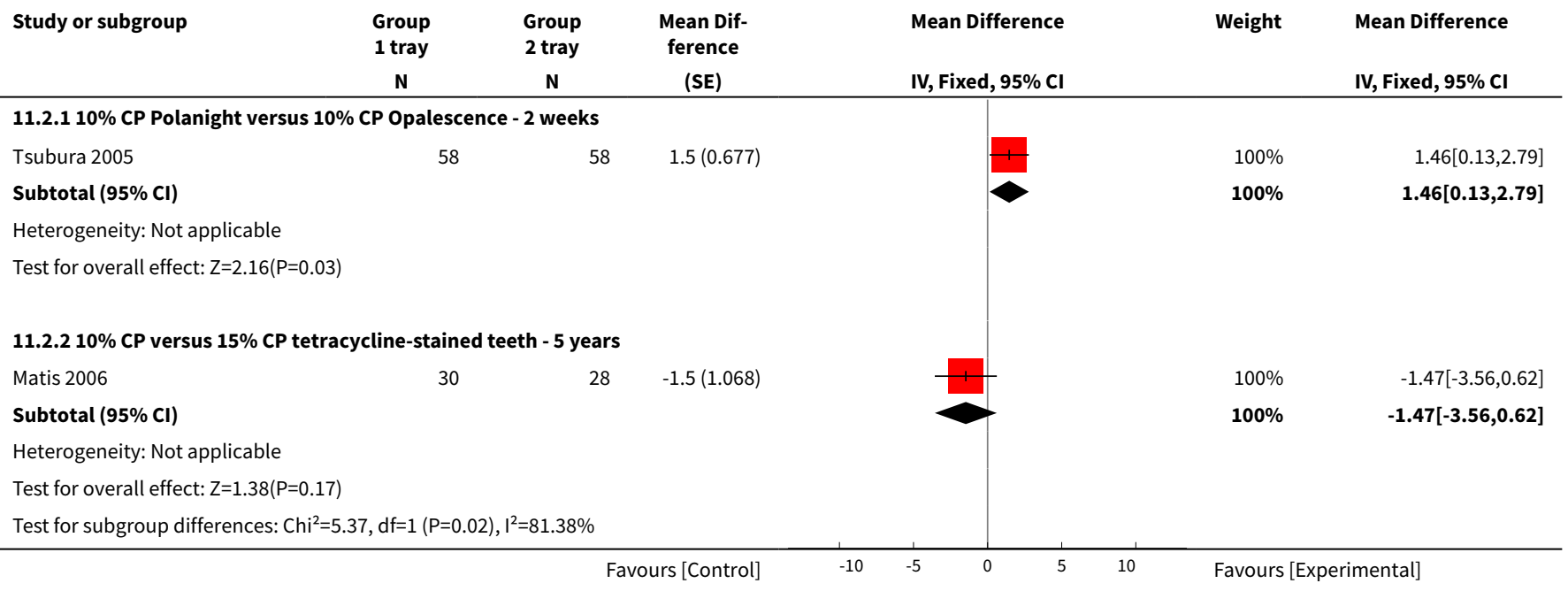

Analysis 11.3. Comparison $11 \mathrm{CP}$ tray versus CP tray, Outcome 3 Tooth whitening - reported by the patient.

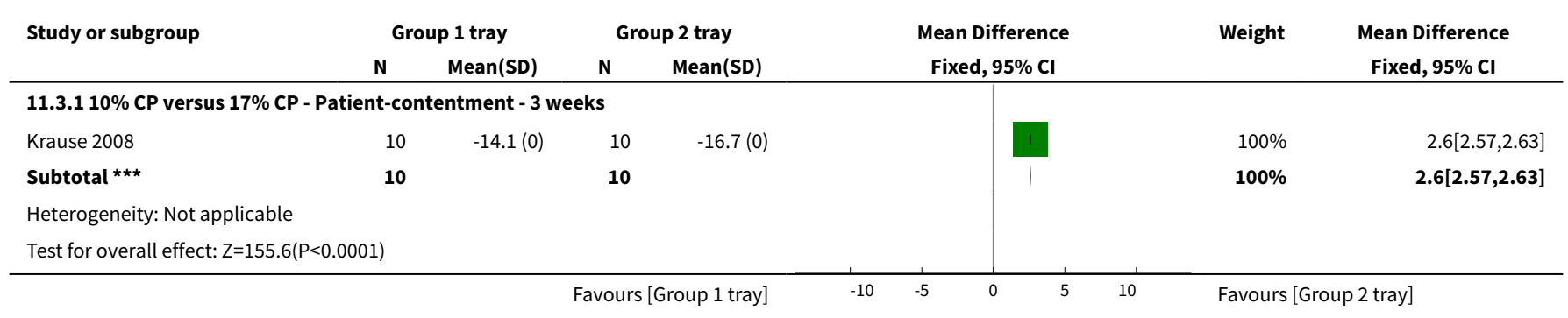




\section{Comparison 12. CP tray versus CP tray}

\begin{tabular}{|c|c|c|c|c|}
\hline Outcome or subgroup title & No. of studies & $\begin{array}{l}\text { No. of partici- } \\
\text { pants }\end{array}$ & Statistical method & Effect size \\
\hline $\begin{array}{l}1 \text { Tooth whitening - assessed by the } \\
\text { dentist }\end{array}$ & 1 & & $\begin{array}{l}\text { Mean Difference (IV, Random, } \\
95 \% \mathrm{CI})\end{array}$ & Subtotals only \\
\hline $1.110 \%$ CP versus $15 \%$ CP - 2 weeks & 1 & 52 & $\begin{array}{l}\text { Mean Difference (IV, Random, } \\
95 \% \mathrm{CI})\end{array}$ & $1.65[0.22,3.08]$ \\
\hline $\begin{array}{l}2 \text { Tooth whitening - assessed by the } \\
\text { dentist }\end{array}$ & 1 & 25 & $\begin{array}{l}\text { Mean Difference (Fixed, 95\% } \\
\mathrm{Cl} \text { ) }\end{array}$ & $2.22[1.29,3.15]$ \\
\hline $2.110 \%$ CP versus $15 \%$ CP - 2 weeks & 1 & 25 & $\begin{array}{l}\text { Mean Difference (Fixed, 95\% } \\
\mathrm{Cl} \text { ) }\end{array}$ & $2.22[1.29,3.15]$ \\
\hline $\begin{array}{l}3 \text { Tooth whitening - assessed by the } \\
\text { dentist }\end{array}$ & 2 & 58 & $\begin{array}{l}\text { Std. Mean Difference (IV, Fixed, } \\
95 \% \mathrm{CI})\end{array}$ & $0.32[-0.20,0.84]$ \\
\hline $\begin{array}{l}3.110 \% \mathrm{CP} \text { versus } 10 \% \mathrm{CP}+\mathrm{KN}+ \\
\mathrm{NaF}-2 \text { weeks }\end{array}$ & 2 & 58 & $\begin{array}{l}\text { Std. Mean Difference (IV, Fixed, } \\
95 \% \mathrm{CI})\end{array}$ & $0.32[-0.20,0.84]$ \\
\hline
\end{tabular}

Analysis 12.1. Comparison 12 CP tray versus CP tray, Outcome 1 Tooth whitening - assessed by the dentist.

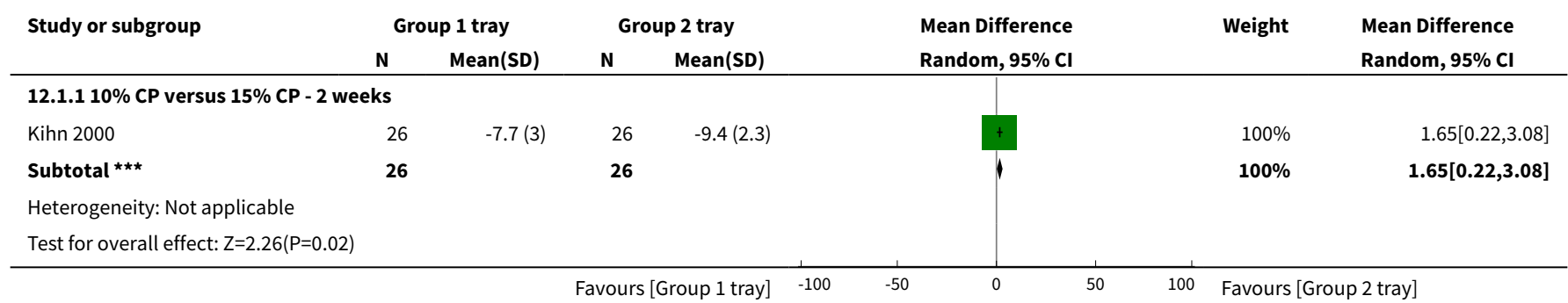

Analysis 12.2. Comparison $12 \mathrm{CP}$ tray versus CP tray, Outcome 2 Tooth whitening - assessed by the dentist.

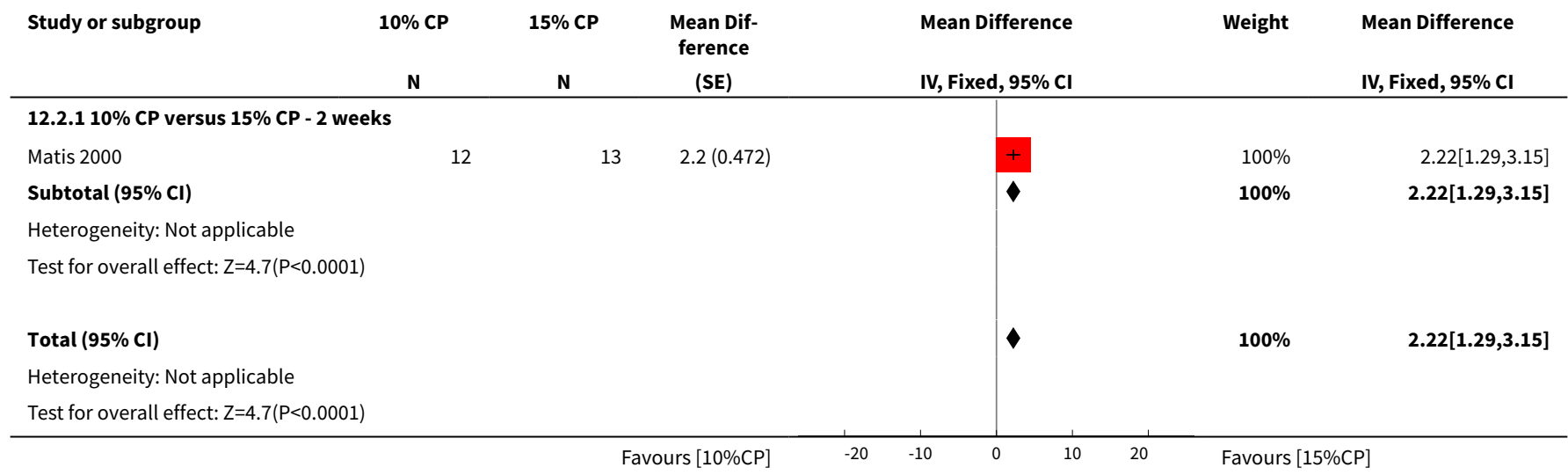


Analysis 12.3. Comparison $12 \mathrm{CP}$ tray versus CP tray, Outcome 3 Tooth whitening - assessed by the dentist.

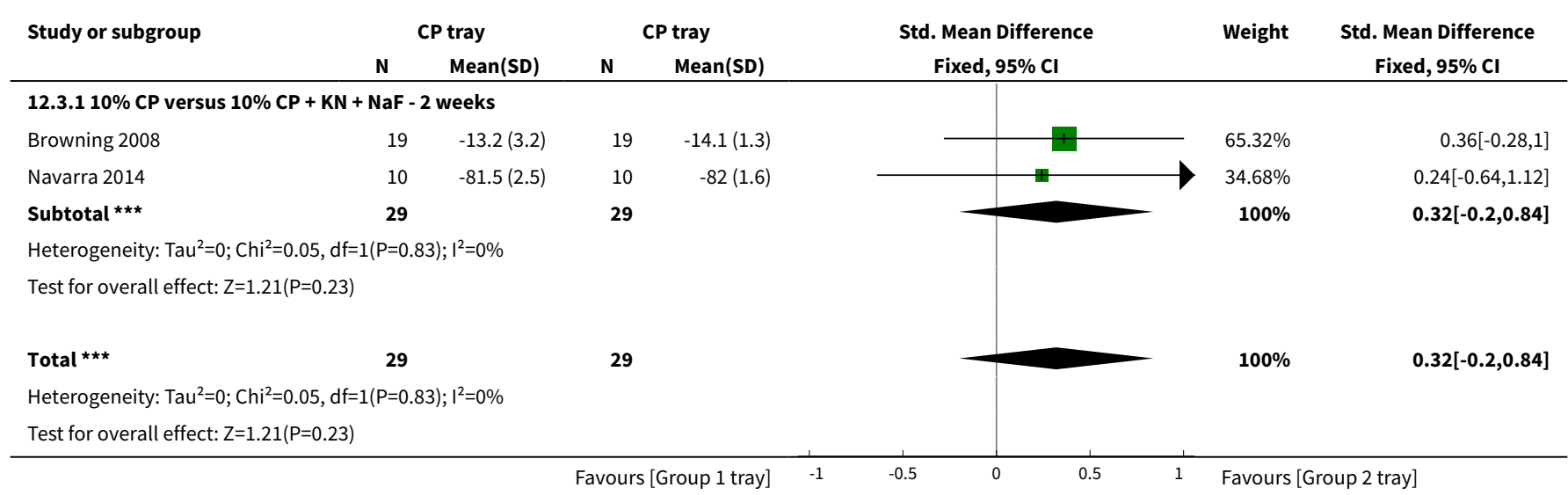

\section{Comparison 13. CP tray versus HP tray}

\begin{tabular}{|c|c|c|c|c|}
\hline Outcome or subgroup title & No. of studies & $\begin{array}{l}\text { No. of partici- } \\
\text { pants }\end{array}$ & Statistical method & Effect size \\
\hline $\begin{array}{l}1 \text { Tooth whitening - assessed by the } \\
\text { dentist }\end{array}$ & 5 & & $\begin{array}{l}\text { Mean Difference (IV, Random, } \\
95 \% \mathrm{CI} \text { ) }\end{array}$ & Subtotals only \\
\hline $1.120 \%$ CP versus $7.5 \%$ HP - 12 days & 1 & 56 & $\begin{array}{l}\text { Mean Difference (IV, Random, } \\
95 \% \mathrm{CI} \text { ) }\end{array}$ & $-0.99[-2.32,0.34]$ \\
\hline $\begin{array}{l}1.210 \% \text { CP versus } 7.5 \% \mathrm{HP} \text { - } 2 \\
\text { weeks }\end{array}$ & 1 & 48 & $\begin{array}{l}\text { Mean Difference (IV, Random, } \\
95 \% \mathrm{Cl} \text { ) }\end{array}$ & $-1.00[-2.86,0.86]$ \\
\hline $1.320 \%$ CP versus $9 \% \mathrm{HP}$ - 2 weeks & 1 & 37 & $\begin{array}{l}\text { Mean Difference (IV, Random, } \\
95 \% \mathrm{CI} \text { ) }\end{array}$ & $-0.58[-8.01,6.85]$ \\
\hline $\begin{array}{l}1.410 \% \mathrm{CP} \text { versus } 6 \% \mathrm{HP} \text { - } 2 \text { weeks - } \\
\text { medium dark and light shade }\end{array}$ & 1 & 349 & $\begin{array}{l}\text { Mean Difference (IV, Random, } \\
95 \% \mathrm{CI} \text { ) }\end{array}$ & $-2.22[-2.63,-1.81]$ \\
\hline $\begin{array}{l}1.510 \% \text { CP versus } 6 \% \mathrm{HP} \text { - } 2 \text { weeks - } \\
\text { darker shade }\end{array}$ & 1 & 164 & $\begin{array}{l}\text { Mean Difference (IV, Random, } \\
95 \% \mathrm{CI} \text { ) }\end{array}$ & $-4.3[-5.02,-3.58]$ \\
\hline $\begin{array}{l}1.620 \% \text { CP versus } 7.5 \% \mathrm{HP}-12 \\
\text { weeks }\end{array}$ & 1 & 24 & $\begin{array}{l}\text { Mean Difference (IV, Random, } \\
95 \% \mathrm{CI} \text { ) }\end{array}$ & $-0.25[-0.40,-0.10]$ \\
\hline $\begin{array}{l}2 \text { Tooth whitening - assessed by the } \\
\text { dentist }\end{array}$ & 1 & & $\begin{array}{l}\text { Mean Difference (Fixed, 95\% } \\
\mathrm{Cl} \text { ) }\end{array}$ & Subtotals only \\
\hline $\begin{array}{l}2.120 \% \text { CP versus } 7.5 \% \text { HP - } 12 \\
\text { weeks }\end{array}$ & 1 & 24 & $\begin{array}{l}\text { Mean Difference (Fixed, 95\% } \\
\mathrm{Cl} \text { ) }\end{array}$ & $0.25[0.10,0.40]$ \\
\hline
\end{tabular}


Analysis 13.1. Comparison $13 \mathrm{CP}$ tray versus HP tray, Outcome 1 Tooth whitening - assessed by the dentist.

\begin{tabular}{|c|c|c|c|c|c|c|c|}
\hline \multirow[t]{2}{*}{ Study or subgroup } & \multicolumn{2}{|c|}{ CP tray } & \multicolumn{2}{|c|}{ HP tray } & \multirow{2}{*}{$\begin{array}{l}\text { Mean Difference } \\
\text { Random, 95\% Cl }\end{array}$} & \multirow[t]{2}{*}{ Weight } & \multirow{2}{*}{$\begin{array}{l}\text { Mean Difference } \\
\text { Random, } 95 \% \mathrm{Cl}\end{array}$} \\
\hline & $\mathbf{N}$ & Mean(SD) & $\mathbf{N}$ & $\operatorname{Mean}(S D)$ & & & \\
\hline \multicolumn{5}{|c|}{ 13.1.1 20\% CP versus $7.5 \%$ HP - 12 days } & & & \\
\hline Ziebolz 2007 & 27 & $-2.6(3.1)$ & 29 & $-1.6(1.8)$ & & $100 \%$ & $-0.99[-2.32,0.34]$ \\
\hline Subtotal $\star \star \star$ & 27 & & 29 & & & $100 \%$ & $-0.99[-2.32,0.34]$ \\
\hline \multicolumn{8}{|c|}{ Heterogeneity: Not applicable } \\
\hline \multicolumn{8}{|c|}{ Test for overall effect: $Z=1.46(P=0.14)$} \\
\hline \multicolumn{8}{|c|}{ 13.1.2 $10 \%$ CP versus $7.5 \%$ HP - 2 weeks } \\
\hline Alonso 2014 & 24 & $3.4(3.7)$ & 24 & $4.4(2.8)$ & & $100 \%$ & $-1[-2.86,0.86]$ \\
\hline Subtotal $\star \star \star ~$ & 24 & & 24 & & & $100 \%$ & $-1[-2.86,0.86]$ \\
\hline \multicolumn{8}{|c|}{ Heterogeneity: Not applicable } \\
\hline \multicolumn{8}{|c|}{ Test for overall effect: $Z=1.06(P=0.29)$} \\
\hline Delgado 2007 & 16 & $-7(10.7)$ & 21 & $-6.4(12.3)$ & & $100 \%$ & $-0.58[-8.01,6.85]$ \\
\hline 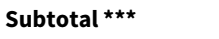 & 16 & & 21 & & & $100 \%$ & $-0.58[-8.01,6.85]$ \\
\hline \multicolumn{8}{|c|}{ Heterogeneity: Not applicable } \\
\hline \multicolumn{8}{|c|}{ Test for overall effect: $Z=0.15(P=0.88)$} \\
\hline \multicolumn{8}{|c|}{ 13.1.4 10\% CP versus $6 \%$ HP - 2 weeks - medium dark and light shade } \\
\hline Aka 2017 & 89 & $-5.9(2)$ & 91 & $-3.8(1.6)$ & + & $60.54 \%$ & $-2.1[-2.63,-1.57]$ \\
\hline Aka 2017 & 81 & $-8.1(2.4)$ & 88 & $-5.7(1.9)$ & $\#$ & $39.46 \%$ & $-2.4[-3.06,-1.74]$ \\
\hline Subtotal $\star \star \star ~$ & 170 & & 179 & & $\diamond$ & $100 \%$ & $-2.22[-2.63,-1.81]$ \\
\hline \multicolumn{8}{|c|}{ Heterogeneity: $\operatorname{Tau}^{2}=0 ; \mathrm{Chi}^{2}=0.49, \mathrm{df}=1(\mathrm{P}=0.49) ; \mathrm{I}^{2}=0 \%$} \\
\hline \multicolumn{8}{|c|}{ Test for overall effect: $Z=10.55(P<0.0001)$} \\
\hline \multicolumn{8}{|c|}{ 13.1.5 10\% CP versus $6 \%$ HP - 2 weeks - darker shade } \\
\hline \multicolumn{8}{|c|}{ Heterogeneity: Not applicable } \\
\hline \multicolumn{8}{|c|}{ Test for overall effect: $Z=11.7(P<0.0001)$} \\
\hline \multicolumn{8}{|c|}{ 13.1.6 20\% CP versus $7.5 \%$ HP - 12 weeks } \\
\hline Mokhlis 2000 & 13 & $-2(0.2)$ & 11 & $-1.7(0.2)$ & + & $100 \%$ & $-0.25[-0.4,-0.1]$ \\
\hline Subtotal $\star \star \star$ & 13 & & 11 & & 1 & $100 \%$ & $-0.25[-0.4,-0.1]$ \\
\hline \multicolumn{8}{|c|}{ Heterogeneity: Not applicable } \\
\hline \multicolumn{8}{|c|}{ Test for overall effect: $\mathrm{Z}=3.21(\mathrm{P}=0)$} \\
\hline Test for subgroup diff & $.01, c$ & $(P<0.0001)$, & $7.24 \%$ & & & & \\
\hline & & & & ars [CP tray] & -5 & Favours & \\
\hline
\end{tabular}

Analysis 13.2. Comparison $13 \mathrm{CP}$ tray versus HP tray, Outcome 2 Tooth whitening - assessed by the dentist.

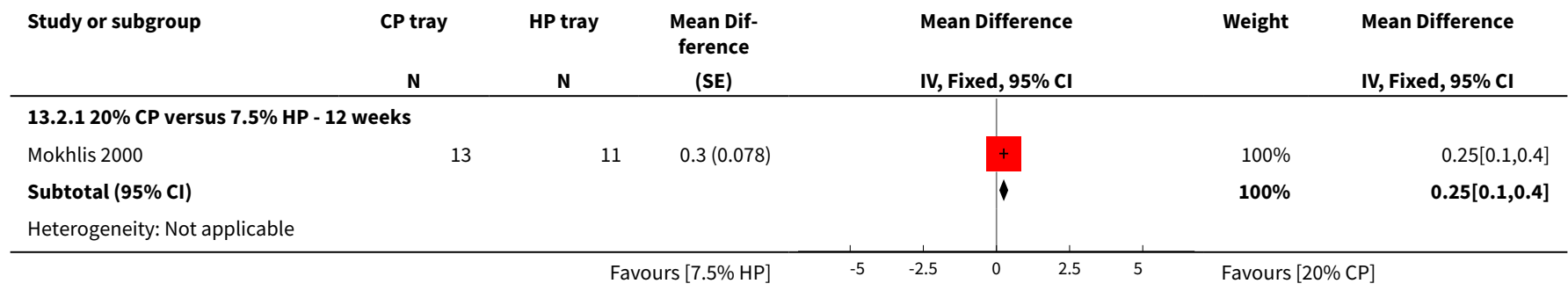




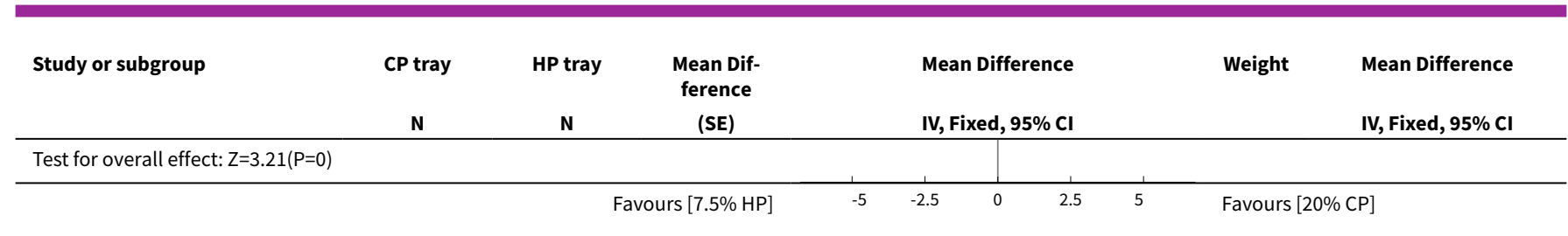

\section{Comparison 14. HP strip versus CP tray}

\begin{tabular}{|c|c|c|c|c|}
\hline Outcome or subgroup title & No. of studies & $\begin{array}{l}\text { No. of partici- } \\
\text { pants }\end{array}$ & Statistical method & Effect size \\
\hline $\begin{array}{l}1 \text { Tooth whitening - assessed by the } \\
\text { dentist }\end{array}$ & 9 & & $\begin{array}{l}\text { Mean Difference (IV, Random, } \\
95 \% \mathrm{Cl} \text { ) }\end{array}$ & Subtotals only \\
\hline $\begin{array}{l}1.16 \% \mathrm{HP} \text { strip versus } 5 \% \mathrm{CP}+5 \% \mathrm{KN} \\
\text { tray - } 1 \text { week }\end{array}$ & 1 & 32 & $\begin{array}{l}\text { Mean Difference (IV, Random, } \\
95 \% \mathrm{CI} \text { ) }\end{array}$ & $-0.71[-1.35,-0.07]$ \\
\hline $\begin{array}{l}1.26 \% \text { HP strip versus } 10 \% \text { CP tray - } 2 \\
\text { weeks }\end{array}$ & 4 & 149 & $\begin{array}{l}\text { Mean Difference (IV, Random, } \\
95 \% \mathrm{Cl} \text { ) }\end{array}$ & $-0.42[-0.92,0.09]$ \\
\hline $\begin{array}{l}1.36 .5 \% \text { HP strip versus } 16 \% \text { CP tray } \\
-21 \text { days }\end{array}$ & 1 & 55 & $\begin{array}{l}\text { Mean Difference (IV, Random, } \\
95 \% \mathrm{CI} \text { ) }\end{array}$ & $2.10[1.16,3.04]$ \\
\hline $\begin{array}{l}1.46 \% \text { HP strip versus } 10 \% \text { CP tray - } 6 \\
\text { weeks }\end{array}$ & 1 & 36 & $\begin{array}{l}\text { Mean Difference (IV, Random, } \\
95 \% \mathrm{CI} \text { ) }\end{array}$ & $-0.30[-0.95,0.35]$ \\
\hline $\begin{array}{l}1.56 .5 \% \text { HP strip versus } 10 \% \text { CP tray - } \\
2 \text { months }\end{array}$ & 1 & 33 & $\begin{array}{l}\text { Mean Difference (IV, Random, } \\
95 \% \mathrm{Cl} \text { ) }\end{array}$ & $-2.63[-4.45,-0.81]$ \\
\hline $\begin{array}{l}1.66 .5 \% \text { HP strip versus } 15 \% \text { CP tray - } \\
3 \text { months }\end{array}$ & 1 & 24 & $\begin{array}{l}\text { Mean Difference (IV, Random, } \\
95 \% \mathrm{CI} \text { ) }\end{array}$ & $3.15[-0.15,6.45]$ \\
\hline $\begin{array}{l}2 \text { Tooth whitening - assessed by the } \\
\text { dentist }\end{array}$ & 1 & & $\begin{array}{l}\text { Mean Difference (Fixed, 95\% } \\
\mathrm{Cl} \text { ) }\end{array}$ & Subtotals only \\
\hline $\begin{array}{l}2.114 \% \text { HP strip versus } 35 \% \text { CP tray - } \\
30 \text { days }\end{array}$ & 1 & 24 & $\begin{array}{l}\text { Mean Difference (Fixed, 95\% } \\
\mathrm{Cl} \text { ) }\end{array}$ & $0.58[-0.61,1.77]$ \\
\hline $\begin{array}{l}3 \text { Tooth whitening - reported by the } \\
\text { patient }\end{array}$ & 1 & 43 & $\begin{array}{l}\text { Mean Difference (IV, Fixed, } \\
95 \% \mathrm{Cl} \text { ) }\end{array}$ & $-0.41[-2.05,1.23]$ \\
\hline
\end{tabular}

Analysis 14.1. Comparison 14 HP strip versus CP tray, Outcome 1 Tooth whitening - assessed by the dentist.

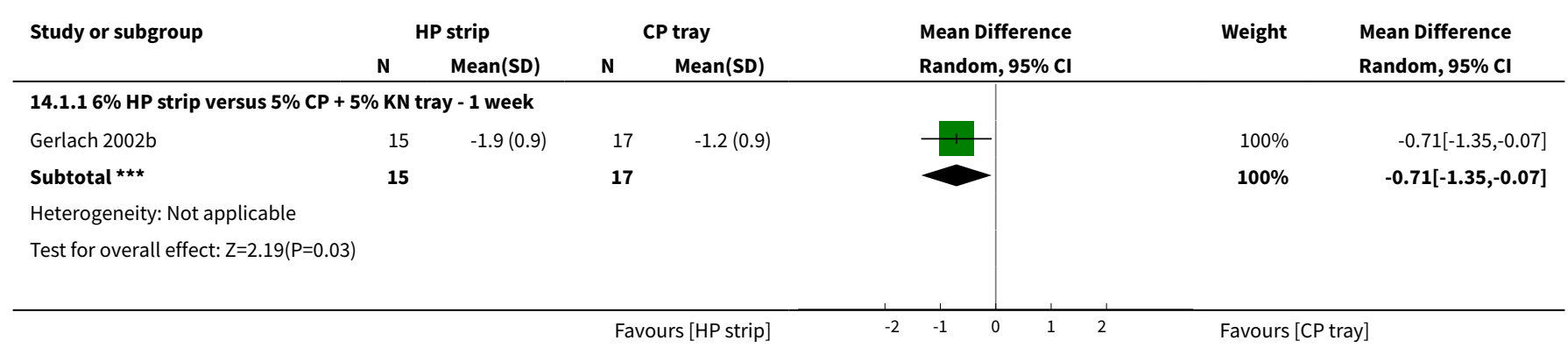




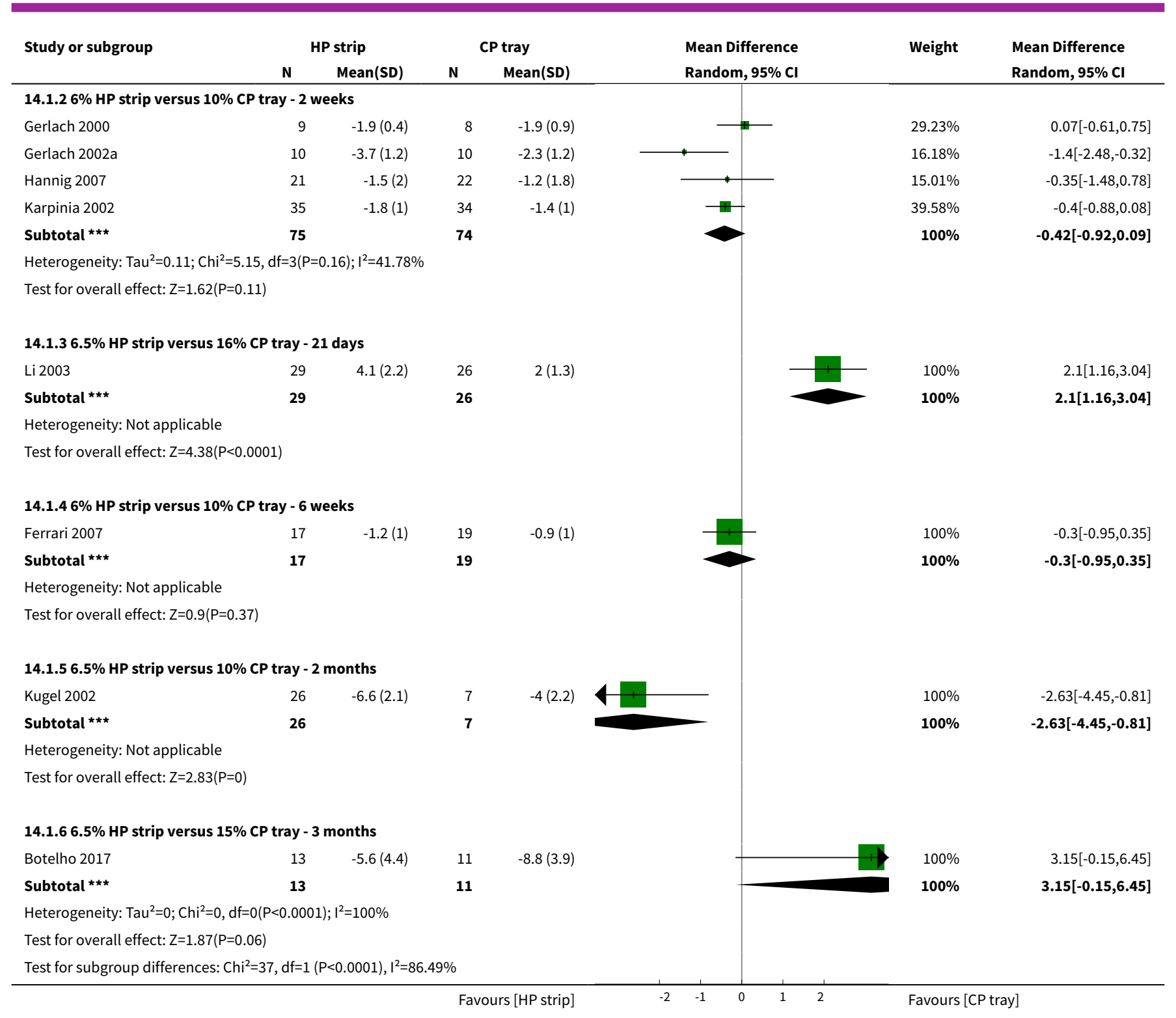

\section{Analysis 14.2. Comparison 14 HP strip versus CP tray, Outcome 2 Tooth whitening - assessed by the dentist.}

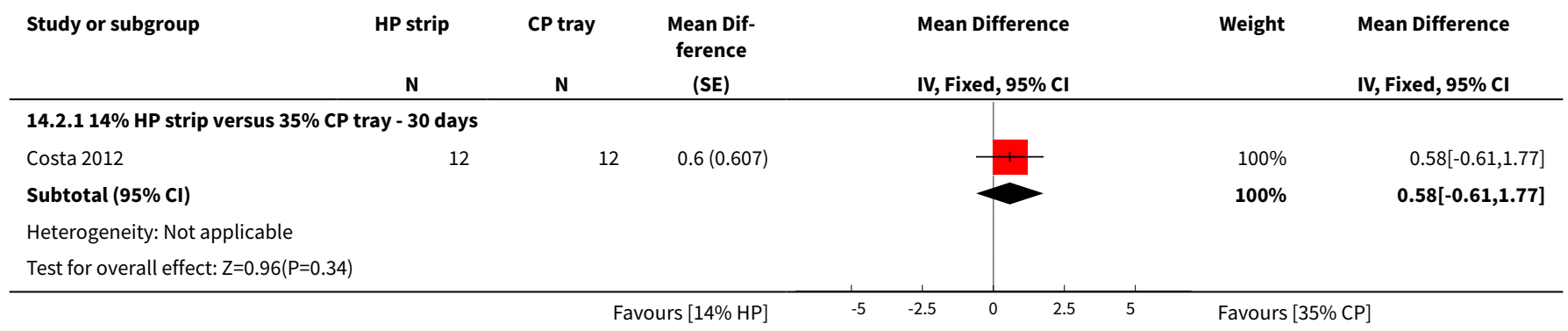


Analysis 14.3. Comparison 14 HP strip versus CP tray, Outcome 3 Tooth whitening - reported by the patient.

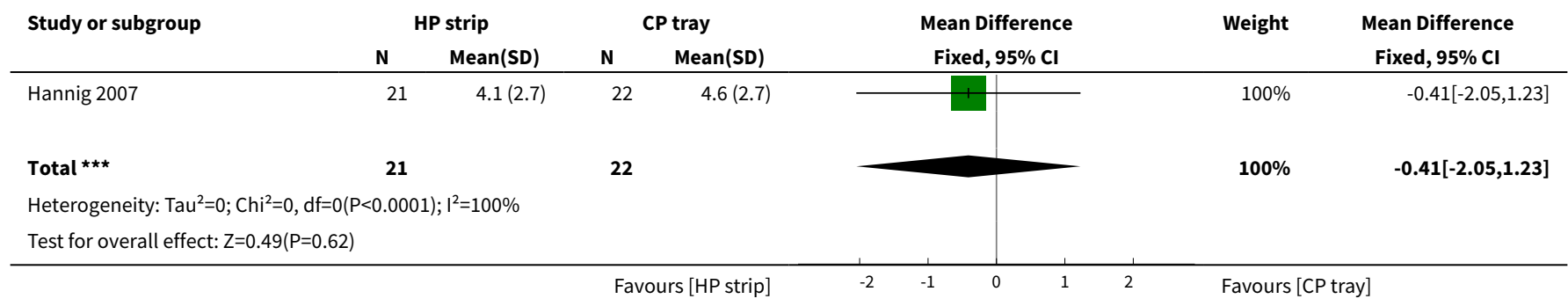

\section{Comparison 15. HP strip versus HP tray}

\begin{tabular}{|c|c|c|c|c|}
\hline Outcome or subgroup title & No. of studies & $\begin{array}{l}\text { No. of partici- } \\
\text { pants }\end{array}$ & Statistical method & Effect size \\
\hline $\begin{array}{l}1 \text { Tooth whitening - assessed by } \\
\text { the dentist }\end{array}$ & 2 & & $\begin{array}{l}\text { Mean Difference (IV, Random, } \\
95 \% \mathrm{CI} \text { ) }\end{array}$ & Subtotals only \\
\hline $\begin{array}{l}1.114 \% \text { HP strip versus } 9.5 \% \mathrm{HP} \\
\text { tray - } 22 \text { days }\end{array}$ & 1 & 29 & $\begin{array}{l}\text { Mean Difference (IV, Random, } \\
95 \% \mathrm{CI} \text { ) }\end{array}$ & $-1.4[-2.35,-0.45]$ \\
\hline $\begin{array}{l}1.25 .3 \% \mathrm{HP} \text { strip versus } 5 \% \mathrm{HP} \\
\text { tray - } 18 \text { months }\end{array}$ & 1 & 28 & $\begin{array}{l}\text { Mean Difference (IV, Random, } \\
95 \% \mathrm{CI})\end{array}$ & $-0.06[-2.36,2.24]$ \\
\hline 2 Patient comfort & 1 & & $\begin{array}{l}\text { Mean Difference (IV, Fixed, 95\% } \\
\mathrm{CI} \text { ) }\end{array}$ & Subtotals only \\
\hline $\begin{array}{l}2.15 .3 \% \text { HP strip versus } 5 \% \text { HP } \\
\text { tray }\end{array}$ & 1 & 28 & $\begin{array}{l}\text { Mean Difference (IV, Fixed, 95\% } \\
\mathrm{CI} \text { ) }\end{array}$ & $1.27[0.13,2.41]$ \\
\hline
\end{tabular}

\section{Analysis 15.1. Comparison 15 HP strip versus HP tray, Outcome 1 Tooth whitening - assessed by the dentist.}

\begin{tabular}{|c|c|c|c|c|c|c|c|}
\hline \multirow[t]{2}{*}{ Study or subgroup } & \multicolumn{2}{|c|}{ HP strip } & \multicolumn{2}{|c|}{ HP tray } & \multirow{2}{*}{$\begin{array}{l}\text { Mean Difference } \\
\text { Random, 95\% Cl }\end{array}$} & \multirow[t]{2}{*}{ Weight } & \multirow{2}{*}{$\begin{array}{l}\text { Mean Difference } \\
\text { Random, } 95 \% \mathrm{CI}\end{array}$} \\
\hline & $\mathbf{N}$ & Mean(SD) & $\mathbf{N}$ & Mean(SD) & & & \\
\hline \multicolumn{8}{|c|}{ 15.1.1 14\% HP strip versus $9.5 \%$ HP tray - 22 days } \\
\hline Gerlach 2004 & 14 & $-3.1(1.3)$ & 15 & $-1.7(1.3)$ & & $100 \%$ & $-1.4[-2.35,-0.45]$ \\
\hline Subtotal $* \star \star$ & 14 & & 15 & & & $100 \%$ & $-1.4[-2.35,-0.45]$ \\
\hline \multicolumn{8}{|c|}{ Heterogeneity: Not applicable } \\
\hline \multicolumn{8}{|c|}{ Test for overall effect: $Z=2.9(P=0)$} \\
\hline \multicolumn{8}{|c|}{ 15.1.2 5.3\% HP strip versus $5 \%$ HP tray - 18 months } \\
\hline Auschill 2012 & 14 & $-6.4(2.7)$ & 14 & $-6.3(3.5)$ & & $100 \%$ & $-0.06[-2.36,2.24]$ \\
\hline Subtotal $* \star \star$ & 14 & & 14 & & & $100 \%$ & $-0.06[-2.36,2.24]$ \\
\hline \multicolumn{8}{|c|}{ Heterogeneity: Not applicable } \\
\hline \multicolumn{8}{|c|}{ Test for overall effect: $Z=0.05(P=0.96)$} \\
\hline \multicolumn{8}{|c|}{ Test for subgroup differences: $\mathrm{Chi}^{2}=1.12, \mathrm{df}=1(\mathrm{P}=0.29), \mathrm{I}^{2}=10.34 \%$} \\
\hline
\end{tabular}


Analysis 15.2. Comparison 15 HP strip versus HP tray, Outcome 2 Patient comfort.

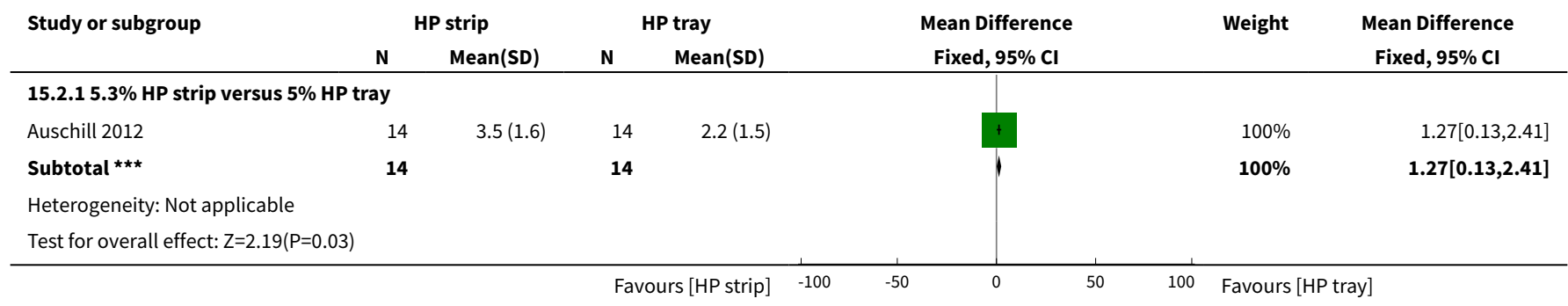

\section{Comparison 16. HP strip versus HP strip}

\begin{tabular}{lllll}
\hline Outcome or subgroup title & No. of studies & $\begin{array}{l}\text { No. of partici- } \\
\text { pants }\end{array}$ & Statistical method & Effect size \\
\hline $\begin{array}{l}1 \text { Tooth whitening - assessed by the } \\
\text { dentist }\end{array}$ & 2 & & $\begin{array}{l}\text { Mean Difference (IV, Random, } \\
95 \% \mathrm{Cl})\end{array}$ & Subtotals only \\
\hline $\begin{array}{l}1.19 .5 \% \text { HP strip versus 10\% HP strip } \\
\text { day } 9\end{array}$ & 1 & 29 & $\begin{array}{l}\text { Mean Difference (IV, Random, } \\
95 \% \mathrm{Cl})\end{array}$ & $-1.5[-2.33,-0.67]$ \\
\hline $\begin{array}{l}1.26 \% \text { HP strip versus 10\% HP strip - } \\
15 \text { days }\end{array}$ & 1 & 35 & $\begin{array}{l}\text { Mean Difference (IV, Random, } \\
95 \% \mathrm{Cl})\end{array}$ & 0.68 [0.16, 1.20] \\
\hline
\end{tabular}

Analysis 16.1. Comparison 16 HP strip versus HP strip, Outcome 1 Tooth whitening - assessed by the dentist.

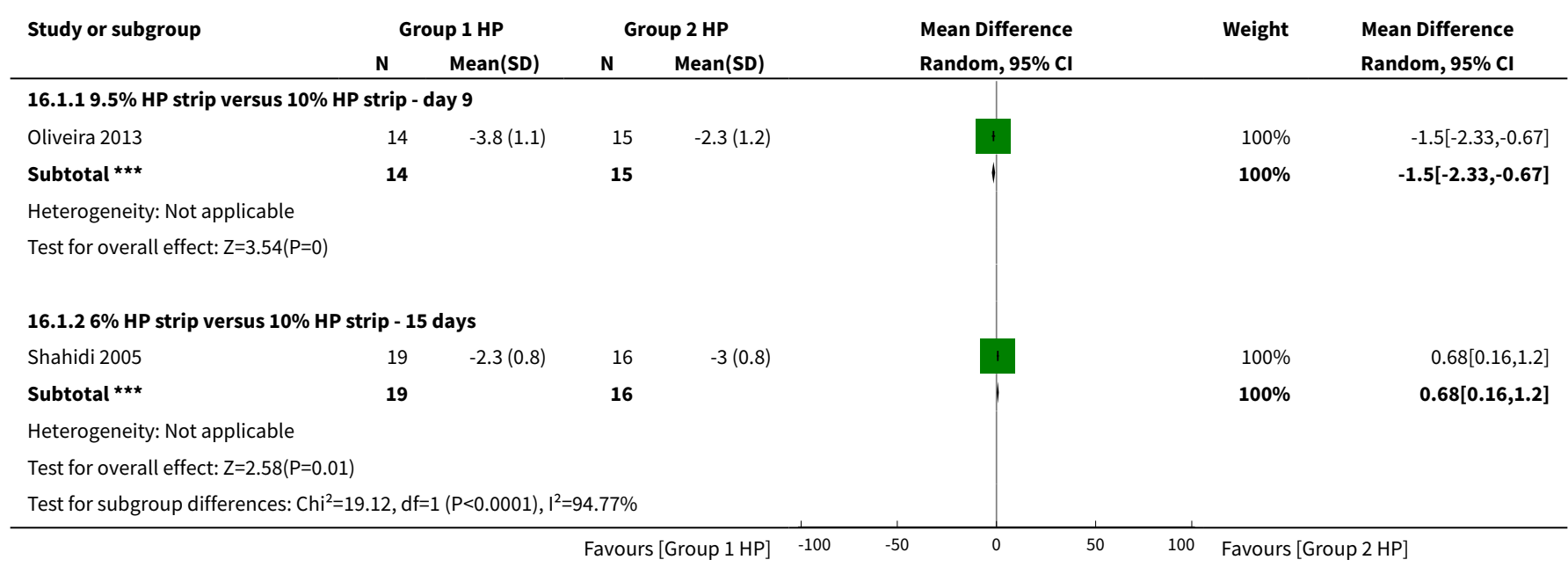


Comparison 17. HP strip versus HP mouthwash

\begin{tabular}{lllll}
\hline Outcome or subgroup title & No. of studies & $\begin{array}{l}\text { No. of partici- } \\
\text { pants }\end{array}$ & Statistical method & Effect size \\
\hline $\begin{array}{l}1 \text { Tooth whitening - assessed by the } \\
\text { dentist }\end{array}$ & 1 & & $\begin{array}{l}\text { Mean Difference (IV, Random, } \\
95 \% \mathrm{Cl})\end{array}$ & Subtotals only \\
\hline $\begin{array}{l}1.110 \% \text { HP strip versus 2\% HP } \\
\text { mouthwash }\end{array}$ & 1 & 28 & $\begin{array}{l}\text { Mean Difference (IV, Random, } \\
95 \% \mathrm{Cl})\end{array}$ & $-1.10[-1.49$, \\
\hline
\end{tabular}

Analysis 17.1. Comparison 17 HP strip versus HP mouthwash, Outcome 1 Tooth whitening - assessed by the dentist.

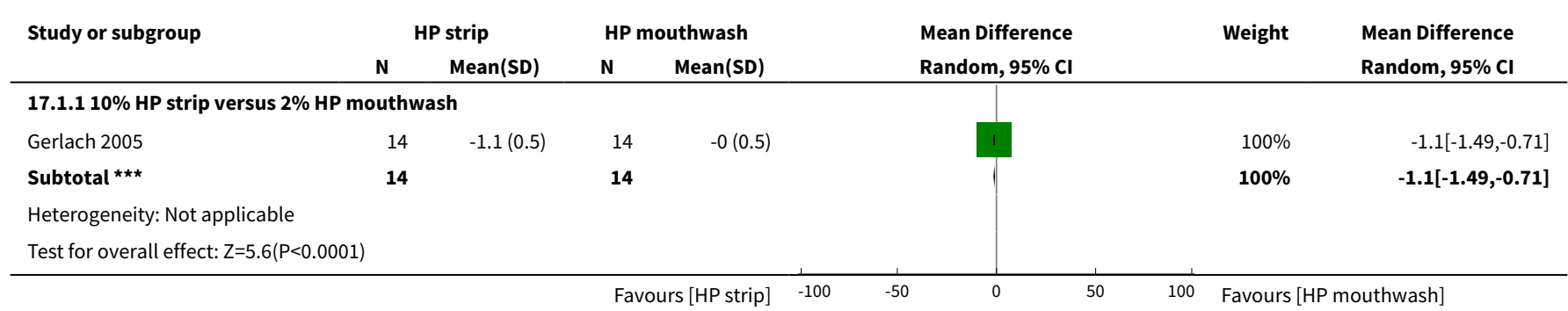

Comparison 18. CP paint-on versus HP strip

\begin{tabular}{lllll}
\hline Outcome or subgroup title & No. of studies & $\begin{array}{l}\text { No. of partici- } \\
\text { pants }\end{array}$ & Statistical method & Effect size \\
\hline $\begin{array}{l}1 \text { Tooth whitening - assessed by } \\
\text { the dentist }\end{array}$ & 2 & & $\begin{array}{l}\text { Std. Mean Difference (IV, Random, } \\
95 \% \text { CI) }\end{array}$ & Subtotals only \\
\hline $\begin{array}{l}1.118 \% \text { CP paint-on versus 6\% HP } \\
\text { strip }\end{array}$ & 2 & 102 & $\begin{array}{l}\text { Std. Mean Difference (IV, Random, } \\
95 \% \text { CI) }\end{array}$ & $1.50[1.06,1.94]$ \\
\hline
\end{tabular}

Analysis 18.1. Comparison $18 \mathrm{CP}$ paint-on versus HP strip, Outcome 1 Tooth whitening - assessed by the dentist.

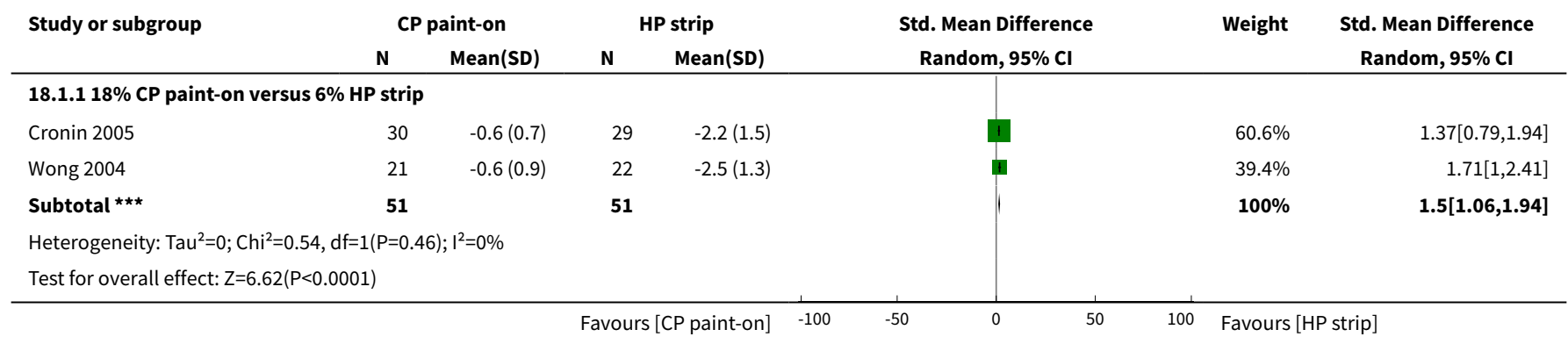


Comparison 19. HP paint-on versus HP strip

\begin{tabular}{|c|c|c|c|c|}
\hline Outcome or subgroup title & No. of studies & $\begin{array}{l}\text { No. of partici- } \\
\text { pants }\end{array}$ & Statistical method & Effect size \\
\hline $\begin{array}{l}1 \text { Tooth whitening - assessed by the } \\
\text { dentist }\end{array}$ & 1 & & $\begin{array}{l}\text { Mean Difference (IV, Random, } \\
95 \% \mathrm{CI})\end{array}$ & Subtotals only \\
\hline $\begin{array}{l}1.15 .9 \% \mathrm{HP} \text { paint-on versus } 6 \% \mathrm{HP} \\
\text { strip }\end{array}$ & 1 & 33 & $\begin{array}{l}\text { Mean Difference (IV, Random, } \\
95 \% \mathrm{CI})\end{array}$ & $1.28[0.77,1.79]$ \\
\hline $\begin{array}{l}2 \text { Tooth whitening - assessed by the } \\
\text { dentist }\end{array}$ & 1 & & $\begin{array}{l}\text { Mean Difference (Fixed, 95\% } \\
\mathrm{Cl} \text { ) }\end{array}$ & Subtotals only \\
\hline $\begin{array}{l}2.15 .9 \% \mathrm{HP} \text { paint-on versus 5.9\% HP } \\
\text { strip }\end{array}$ & 1 & 40 & $\begin{array}{l}\text { Mean Difference (Fixed, 95\% } \\
\mathrm{Cl} \text { ) }\end{array}$ & $2.7[2.08,3.32]$ \\
\hline $\begin{array}{l}3 \text { Tooth whitening - reported by the pa- } \\
\text { tient }\end{array}$ & 1 & & $\begin{array}{l}\text { Mean Difference (IV, Fixed, } \\
95 \% \mathrm{CI} \text { ) }\end{array}$ & Subtotals only \\
\hline $\begin{array}{l}3.15 .9 \% \text { HP paint-on versus 5.9\% HP } \\
\text { strip }\end{array}$ & 1 & 40 & $\begin{array}{l}\text { Mean Difference (IV, Fixed, } \\
95 \% \mathrm{CI})\end{array}$ & $-0.25[-1.88,1.38]$ \\
\hline
\end{tabular}

Analysis 19.1. Comparison 19 HP paint-on versus HP strip, Outcome 1 Tooth whitening - assessed by the dentist.

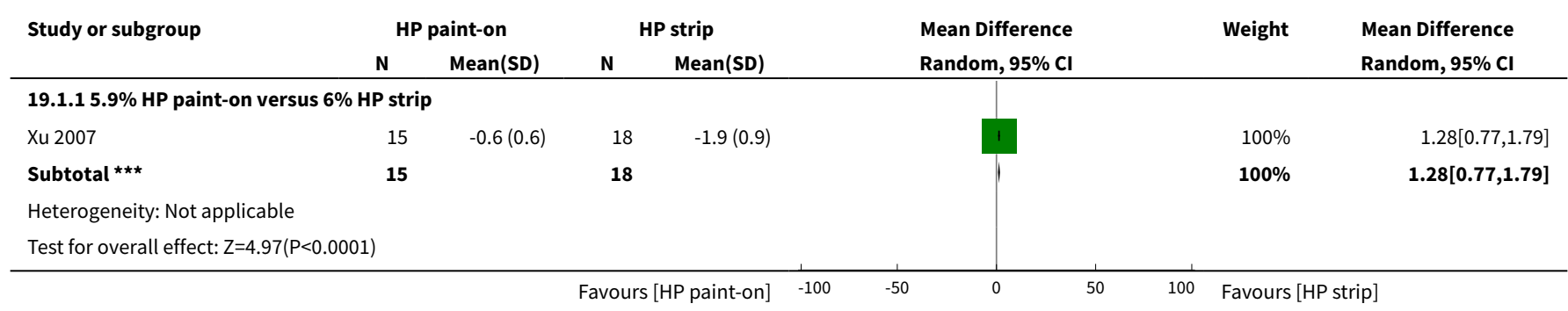

\section{Analysis 19.2. Comparison 19 HP paint-on versus HP strip, Outcome 2 Tooth whitening - assessed by the dentist.}

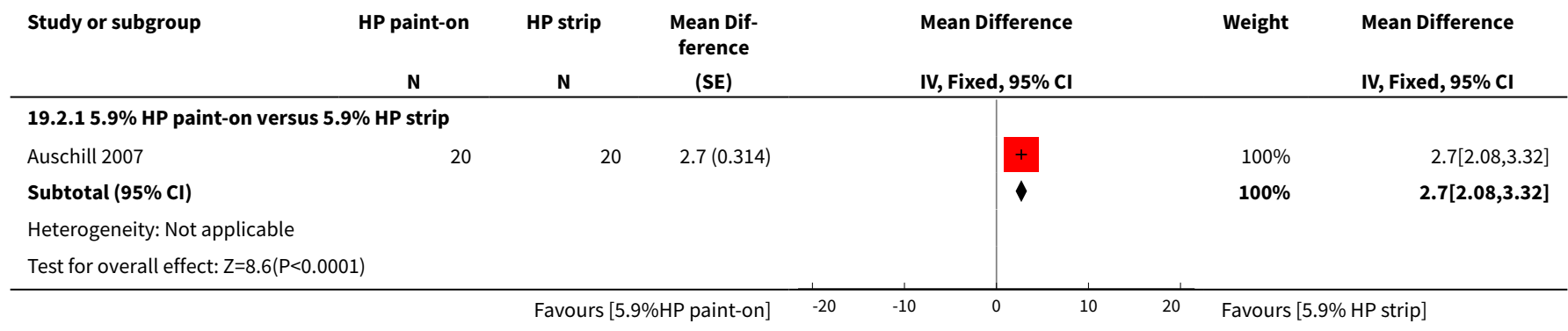


Analysis 19.3. Comparison 19 HP paint-on versus HP strip, Outcome 3 Tooth whitening - reported by the patient.

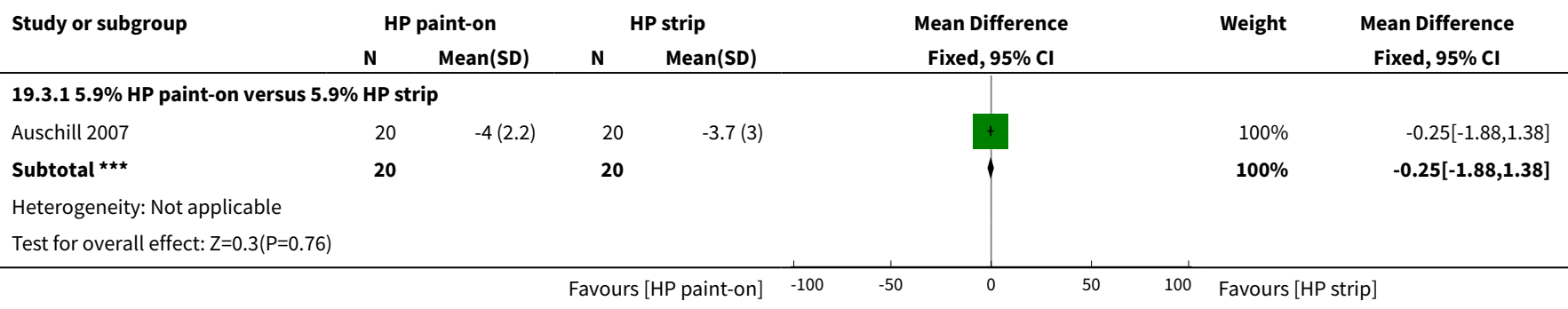

\section{Comparison 20. SPC paint-on versus HP strip}

\begin{tabular}{lllll}
\hline Outcome or subgroup title & No. of studies & $\begin{array}{l}\text { No. of partici- } \\
\text { pants }\end{array}$ & Statistical method & Effect size \\
\hline $\begin{array}{l}1 \text { Tooth whitening - assessed by the } \\
\text { dentist }\end{array}$ & 1 & & $\begin{array}{l}\text { Mean Difference (IV, Random, } \\
95 \% \text { Cl) }\end{array}$ & Subtotals only \\
\hline $\begin{array}{l}1.119 \% \text { SPC paint-on versus 6\% HP } \\
\text { strip - 6 months }\end{array}$ & 1 & 47 & $\begin{array}{l}\text { Mean Difference (IV, Random, } \\
95 \% \text { CI) }\end{array}$ & $0.93[0.59,1.27]$ \\
\hline
\end{tabular}

Analysis 20.1. Comparison 20 SPC paint-on versus HP strip, Outcome 1 Tooth whitening - assessed by the dentist.

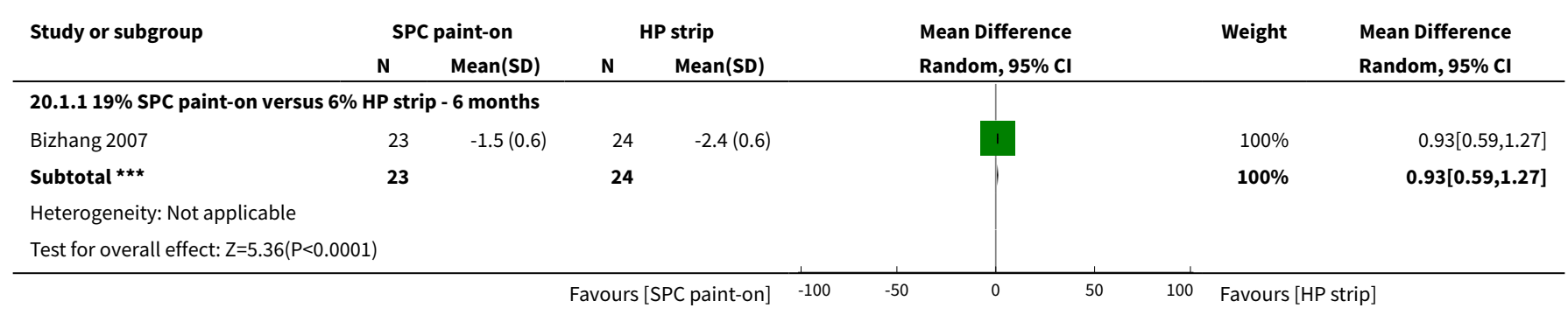

\section{Comparison 21. CP paint-on versus $\mathrm{CP}$ paint-on}

\begin{tabular}{lllll}
\hline Outcome or subgroup title & No. of studies & $\begin{array}{l}\text { No. of partici- } \\
\text { pants }\end{array}$ & Statistical method & Effect size \\
\hline $\begin{array}{l}1 \text { Tooth whitening - assessed by the } \\
\text { dentist }\end{array}$ & 2 & & $\begin{array}{l}\text { Mean Difference (IV, Random, } \\
95 \% \text { Cl) }\end{array}$ & Subtotals only \\
\hline $\begin{array}{l}1.118 \% \text { CP 2x versus 18\% CP 4x-1 } \\
\text { week }\end{array}$ & 1 & 69 & $\begin{array}{l}\text { Mean Difference (IV, Random, } \\
95 \% \text { Cl) }\end{array}$ & 1.39 [0.50, 2.28] \\
\hline $\begin{array}{l}1.218 \% \text { CP versus } 16.4 \% \text { CP - } 2 \\
\text { weeks }\end{array}$ & 1 & 93 & $\begin{array}{l}\text { Mean Difference (IV, Random, } \\
95 \% \text { Cl) }\end{array}$ & $-0.70[-2.21,0.81]$ \\
\hline
\end{tabular}


Analysis 21.1. Comparison 21 CP paint-on versus CP paint-on, Outcome 1 Tooth whitening - assessed by the dentist.

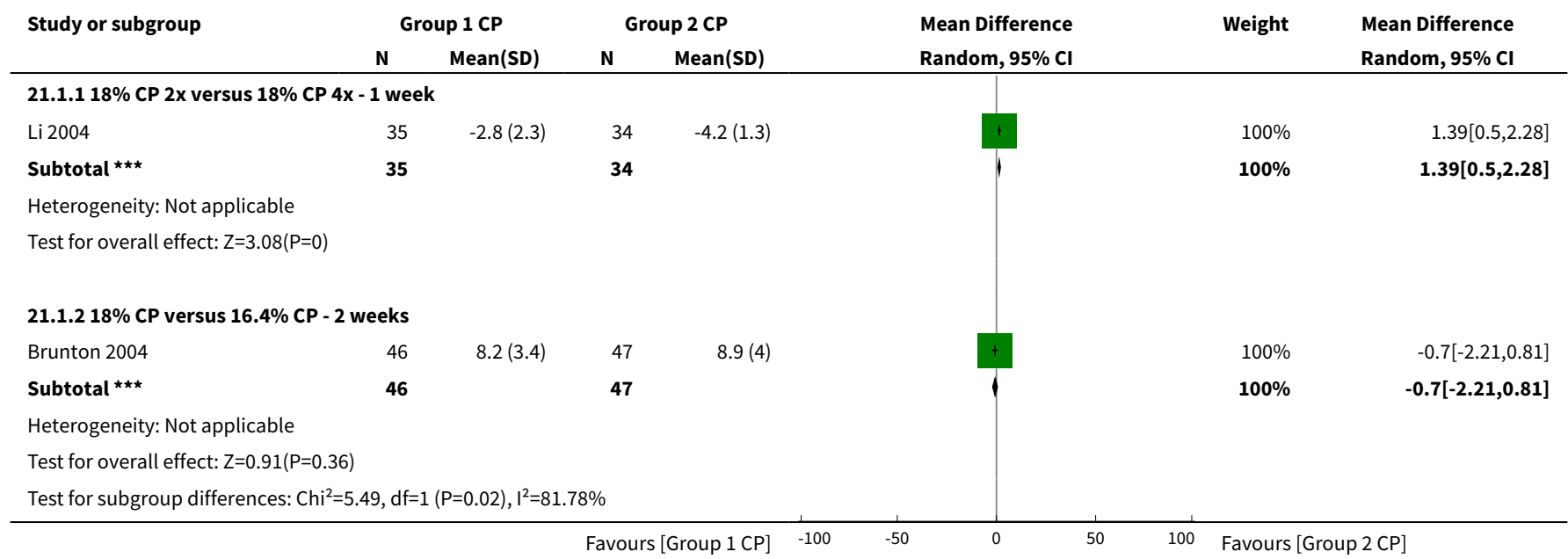

Comparison 22. CP paint-on versus HP paint-on

\begin{tabular}{lllll}
\hline Outcome or subgroup title & No. of studies & $\begin{array}{l}\text { No. of partici- } \\
\text { pants }\end{array}$ & Statistical method & Effect size \\
\hline $\begin{array}{l}1 \text { Tooth whitening - assessed by the } \\
\text { dentist }\end{array}$ & 1 & & $\begin{array}{l}\text { Mean Difference (IV, Random, } \\
95 \% \mathrm{Cl})\end{array}$ & Subtotals only \\
\hline $\begin{array}{l}1.125 \% \text { CP paint-on versus 8.75\% HP } \\
\text { paint-on }\end{array}$ & 1 & 59 & $\begin{array}{l}\text { Mean Difference (IV, Random, } \\
95 \% \mathrm{Cl})\end{array}$ & $-0.16[-1.39,1.07]$ \\
\hline
\end{tabular}

Analysis 22.1. Comparison 22 CP paint-on versus HP paint-on, Outcome 1 Tooth whitening - assessed by the dentist.

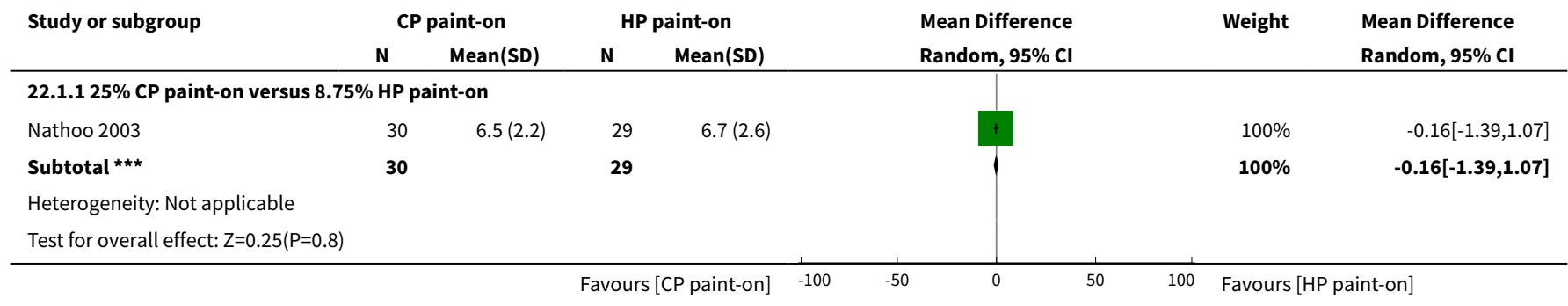

\section{Comparison 23. HP paint-on versus HP paint-on}

\begin{tabular}{lllll}
\hline Outcome or subgroup title & No. of studies & $\begin{array}{l}\text { No. of partici- } \\
\text { pants }\end{array}$ & Statistical method & Effect size \\
\hline $\begin{array}{l}1 \text { Tooth whitening - assessed by the } \\
\text { dentist }\end{array}$ & 1 & & $\begin{array}{l}\text { Mean Difference (IV, Random, } \\
95 \% \mathrm{Cl})\end{array}$ & Subtotals only \\
\hline
\end{tabular}




\begin{tabular}{lllll}
\hline Outcome or subgroup title & No. of studies & $\begin{array}{l}\text { No. of partici- } \\
\text { pants }\end{array}$ & Statistical method & Effect size \\
\hline $\begin{array}{l}1.16 \% \text { HP paint-on versus 6\% HP + KF } \\
\text { paint-on }\end{array}$ & 1 & 67 & $\begin{array}{l}\text { Mean Difference (IV, Random, } \\
95 \% \mathrm{Cl})\end{array}$ & $-0.10[-0.56,0.36]$ \\
\hline
\end{tabular}

Analysis 23.1. Comparison 23 HP paint-on versus HP paint-on, Outcome 1 Tooth whitening - assessed by the dentist.

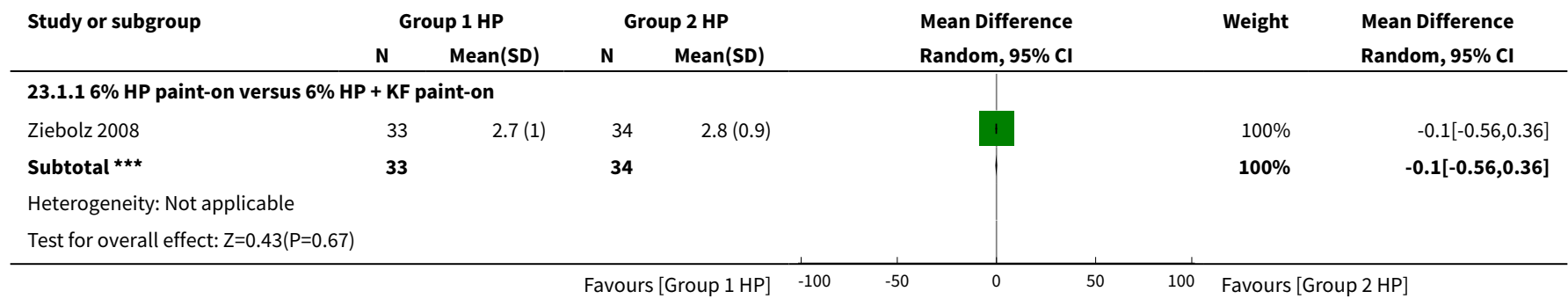

\section{Comparison 24. SPC paint-on versus CP paint-on}

\begin{tabular}{lllll}
\hline Outcome or subgroup title & No. of studies & $\begin{array}{l}\text { No. of partici- } \\
\text { pants }\end{array}$ & Statistical method & Effect size \\
\hline $\begin{array}{l}1 \text { Tooth whitening - assessed by the } \\
\text { dentist }\end{array}$ & 1 & & $\begin{array}{l}\text { Mean Difference (IV, Random, } \\
95 \% \mathrm{Cl})\end{array}$ & Subtotals only \\
\hline $\begin{array}{l}1.119 \% \text { SPC paint-on versus 18\% CP } \\
\text { paint-on - 14 days }\end{array}$ & 1 & 38 & $\begin{array}{l}\text { Mean Difference (IV, Random, } \\
95 \% \mathrm{Cl})\end{array}$ & $-0.58[-0.95$, \\
\hline
\end{tabular}

Analysis 24.1. Comparison 24 SPC paint-on versus CP painton, Outcome 1 Tooth whitening - assessed by the dentist.

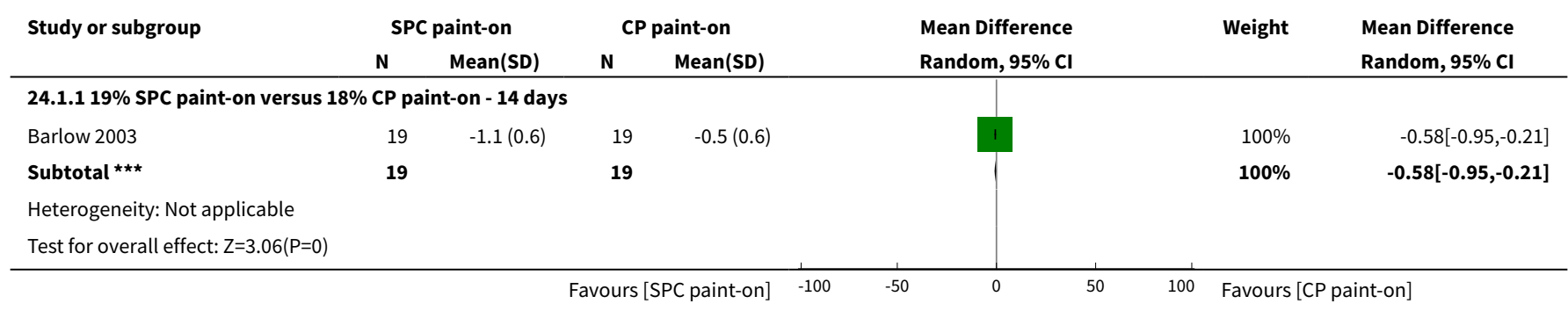


Comparison 25. SPC paint-on versus HP paint-on

\begin{tabular}{lllll}
\hline Outcome or subgroup title & No. of studies & $\begin{array}{l}\text { No. of partici- } \\
\text { pants }\end{array}$ & Statistical method & Effect size \\
\hline $\begin{array}{l}1 \text { Tooth whitening - assessed by the } \\
\text { dentist }\end{array}$ & 1 & & $\begin{array}{l}\text { Mean Difference (IV, Random, } \\
95 \% \mathrm{Cl})\end{array}$ & Subtotals only \\
\hline $\begin{array}{l}1.119 \% \text { SPC paint-on versus 8.7\% HP } \\
\text { paint-on }\end{array}$ & 1 & 56 & Mean Difference (IV, Random, & $-0.36[-0.71$, \\
\hline
\end{tabular}

Analysis 25.1. Comparison 25 SPC paint-on versus HP painton, Outcome 1 Tooth whitening - assessed by the dentist.

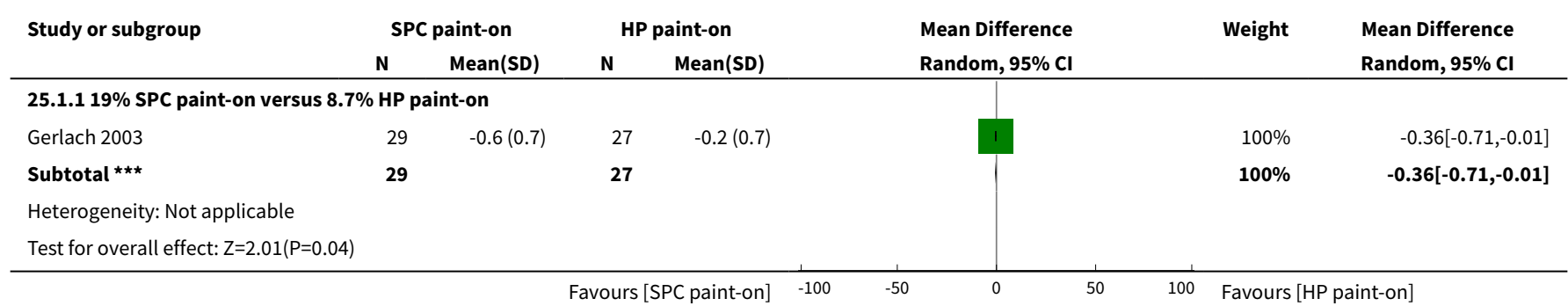

\section{ADDITIONAL TABLES}

Table 1. Details of analyses performed in multiarm trials

\begin{tabular}{|c|c|c|c|}
\hline Trial & Interventions reported in the trials & Interventions considered in analyses & Reason \\
\hline Aka 2017 & $\begin{array}{l}\text { - } \text { Placebo } \\
\text { - } 10 \% \text { carbamide peroxide (Opales- } \\
\text { cence PF) gel in tray } \\
\text { - } 6 \% \text { hydrogen peroxide (Opales- } \\
\text { cence Go) gel in tray }\end{array}$ & $\begin{array}{l}\text { Bleaching agent vs placebo } \\
\text { - } \begin{array}{l}10 \% \text { carbamide peroxide gel in tray vs } \\
\text { placebo }\end{array} \\
\text { Bleaching agent vs bleaching agent } \\
\text { - } 10 \% \text { carbamide peroxide gel in tray vs } 6 \% \\
\text { hydrogen peroxide gel in tray }\end{array}$ & $\begin{array}{l}\text { Most commonly } \\
\text { used concentra- } \\
\text { tions }\end{array}$ \\
\hline Alonso 2014 & $\begin{array}{l}\text { - } 10 \% \text { carbamide peroxide in tray } \\
\text { - } 15 \% \text { carbamide peroxide in tray } \\
\text { - } 7.5 \% \text { hydrogen peroxide in tray } \\
\text { - } 9.5 \% \text { hydrogen peroxide in tray }\end{array}$ & $\begin{array}{l}\text { Bleaching agent vs bleaching agent } \\
\text { - } 10 \% \text { carbamide peroxide in tray vs } 7.5 \% \text { hy- } \\
\text { drogen peroxide in tray }\end{array}$ & $\begin{array}{l}\text { Most commonly } \\
\text { used concentra- } \\
\text { tions }\end{array}$ \\
\hline Bizhang 2007 & $\begin{array}{l}\text { - } 6 \% \text { hydrogen peroxide whitening } \\
\text { strips } \\
\text { - } 19 \% \text { sodium percarbonate brush- } \\
\text { applied gel that dries to a film } \\
\text { - Placebo brush-applied gel without } \\
\text { peroxide }\end{array}$ & $\begin{array}{l}\text { Bleaching agent vs placebo } \\
\text { - } 6 \% \text { hydrogen peroxide whitening strips vs } \\
\text { placebo } \\
\text { Bleaching agent vs bleaching agent }\end{array}$ & $\begin{array}{l}\text { Most commonly } \\
\text { used concentra- } \\
\text { tions }\end{array}$ \\
\hline
\end{tabular}


Table 1. Details of analyses performed in multiarm trials (Continued)

- $19 \%$ sodium percarbonate brush-applied gel that dries to a film vs $6 \%$ hydrogen peroxide whitening strips

\begin{tabular}{|c|c|c|c|}
\hline Browning 2008 & $\begin{array}{l}\text { - } 10 \% \text { carbamide peroxide in tray } \\
\text { - } 10 \% \text { carbamide peroxide, } 3 \% \\
\text { potassium nitrate in tray } \\
\text { - } 10 \% \text { carbamide peroxide, } 0.5 \% \\
\text { potassium nitrate in tray } \\
\text { - } 10 \% \text { carbamide peroxide, } 0.5 \% \\
\text { potassium nitrate, } 0.25 \% \text { sodium } \\
\text { fluoride in tray } \\
\text { - Placebo }\end{array}$ & $\begin{array}{l}\text { Bleaching agent vs placebo } \\
\text { - } 10 \% \text { carbamide peroxide, } 0.5 \% \text { potassium } \\
\text { nitrate, } 0.25 \% \text { sodium fluoride in tray vs } \\
\text { placebo } \\
\text { Bleaching agent vs bleaching agent } \\
\text { - } 10 \% \text { carbamide peroxide in tray vs } 10 \% \text { car- } \\
\text { bamide peroxide, } 0.5 \% \text { potassium nitrate, } \\
0.25 \% \text { sodium fluoride in tray }\end{array}$ & $\begin{array}{l}\text { Most commonly } \\
\text { used concentra- } \\
\text { tions }\end{array}$ \\
\hline Gerlach 2000 & $\begin{array}{l}\text { - } 5.3 \% \text { hydrogen peroxide strips } \\
\text { - } 10 \% \text { carbamide peroxide gel in tray } \\
\text { - } 15 \% \text { carbamide peroxide gel in tray } \\
\text { - } 20 \% \text { carbamide peroxide gel in tray }\end{array}$ & $\begin{array}{l}\text { Bleaching agent vs bleaching agent } \\
\text { - } 5.3 \% \text { hydrogen peroxide strips vs } 10 \% \text { car- } \\
\text { bamide peroxide gel in tray }\end{array}$ & $\begin{array}{l}\text { Most commonly } \\
\text { used concentra- } \\
\text { tions }\end{array}$ \\
\hline Hyland 2015 & $\begin{array}{l}\text { - } 5 \% \text { carbamide peroxide gel } \\
\text { - } 10 \% \text { carbamide peroxide gel } \\
\text { - Placebo }\end{array}$ & $\begin{array}{l}\text { Bleaching agent vs placebo } \\
\text { - } 5 \% \text { carbamide peroxide gel vs placebo } \\
\text { Bleaching agent vs bleaching agent } \\
\text { - } 5 \% \text { carbamide peroxide gel vs } 10 \% \text { car- } \\
\text { bamide peroxide gel }\end{array}$ & $\begin{array}{l}\text { Most commonly } \\
\text { used concentra- } \\
\text { tions }\end{array}$ \\
\hline Krause 2008 & $\begin{array}{l}\text { - } 10 \% \text { carbamide peroxide gel in tray } \\
\text { - } 17 \% \text { carbamide peroxide gel in tray } \\
\text { - } 0 \% \text { carbamide peroxide gel in tray } \\
\text { (control) }\end{array}$ & $\begin{array}{l}\text { Bleaching agent vs bleaching agent } \\
\text { - } 10 \% \text { carbamide peroxide gel in tray vs } 17 \% \\
\text { carbamide peroxide gel in tray }\end{array}$ & $\begin{array}{l}\text { Most commonly } \\
\text { used concentra- } \\
\text { tions }\end{array}$ \\
\hline Li 2003 & $\begin{array}{l}\text { - } 6.5 \% \text { hydrogen peroxide strips } \\
\text { - } 7.5 \% \text { hydrogen peroxide gel in tray } \\
\text { - } 16 \% \text { carbamide peroxide gel in tray }\end{array}$ & $\begin{array}{l}\text { Bleaching agent vs bleaching agent } \\
\text { - } 6.5 \% \text { hydrogen peroxide strips vs } 16 \% \text { car- } \\
\text { bamide peroxide gel in tray }\end{array}$ & $\begin{array}{l}\text { Most commonly } \\
\text { used concentra- } \\
\text { tions }\end{array}$ \\
\hline Li 2004 & $\begin{array}{l}\text { - } 18 \% \text { carbamide peroxide } 2 x \text { paint- } \\
\text { on gel } \\
\text { - } 18 \% \text { carbamide peroxide } 3 x \text { paint- } \\
\text { on gel } \\
\text { - } 18 \% \text { carbamide peroxide } 4 x \text { paint- } \\
\text { on gel }\end{array}$ & $\begin{array}{l}\text { Bleaching agent vs bleaching agent } \\
\text { - } 18 \% \text { carbamide peroxide } 2 x \text { paint-on gel vs } \\
18 \% \text { carbamide peroxide } 4 x \text { paint-on gel }\end{array}$ & $\begin{array}{l}\text { Most commonly } \\
\text { used concentra- } \\
\text { tions }\end{array}$ \\
\hline Matis 2006 & $\begin{array}{l}\text { - } 10 \% \text { carbamide peroxide gel in tray } \\
\text { - } 15 \% \text { carbamide peroxide gel in tray } \\
\text { - } 20 \% \text { carbamide peroxide gel in tray }\end{array}$ & $\begin{array}{l}\text { Bleaching agent vs bleaching agent } \\
\text { - } 10 \% \text { carbamide peroxide gel in tray vs } 15 \% \\
\text { carbamide peroxide gel in tray }\end{array}$ & $\begin{array}{l}\text { Most commonly } \\
\text { used concentra- } \\
\text { tions }\end{array}$ \\
\hline Wong 2004 & $\begin{array}{l}\text { - } 6 \% \text { hydrogen peroxide strips } \\
\text { - } 18 \% \text { carbamide peroxide paint-on } \\
\text { gel } \\
\text { - Placebo: non-whitening tooth- } \\
\text { paste }\end{array}$ & $\begin{array}{l}\text { Bleaching vs placebo } \\
\text { - } 6 \% \text { hydrogen peroxide strips vs placebo } \\
\text { Bleaching agent vs bleaching agent } \\
\text { - } 6 \% \text { hydrogen peroxide strips vs } 18 \% \text { car- } \\
\text { bamide peroxide paint-on gel }\end{array}$ & $\begin{array}{l}\text { Most commonly } \\
\text { used concentra- } \\
\text { tions }\end{array}$ \\
\hline
\end{tabular}


Table 1. Details of analyses performed in multiarm trials (Continued)

\begin{tabular}{|c|c|c|c|}
\hline Xu 2007 & $\begin{array}{l}\text { - } 6 \% \text { hydrogen peroxide strips } \\
\text { - } 5.8 \% \text { hydrogen peroxide paint-on } \\
\text { gel } \\
\text { - Placebo: negative control (water } \\
\text { rinse) }\end{array}$ & $\begin{array}{l}\text { Bleaching vs placebo } \\
\text { - } 6 \% \text { hydrogen peroxide strips vs placebo } \\
\text { Bleaching agent vs bleaching agent } \\
\text { - } 5.8 \% \text { hydrogen peroxide paint-on gel vs } 6 \% \\
\text { hydrogen peroxide strips }\end{array}$ & $\begin{array}{l}\text { Most commonly } \\
\text { used concentra- } \\
\text { tions }\end{array}$ \\
\hline
\end{tabular}

vs = versus; $2 \mathrm{x}=$ twice; $3 \mathrm{x}=3$ times; $4 \mathrm{x}=4$ times.

\section{Table 2. Comparison of included articles: 2006 review version versus current version}

The 2006 review included 25 trials of which 17 trials were included in our review, 3 are under awaiting classification, 2 articles were excluded with reason, 2 were discarded during the initial screening, and 1 did not appear in our search and we could not obtain a copy of:

\begin{tabular}{|c|c|c|c|}
\hline 2006 review & Current review & & \\
\hline Included records & Included records & $\begin{array}{l}\text { Awaiting classifica- } \\
\text { tion }\end{array}$ & No longer included \\
\hline Barnes 1998 & Brunton 2004 & \multirow{21}{*}{$\begin{array}{l}\text { Barnes 1998: unclear } \\
\text { if the trial is a ran- } \\
\text { domised controlled } \\
\text { trial } \\
\text { Gerlach 2004b: full- } \\
\text { text not available } \\
\text { Sielski 2003: full-text } \\
\text { not available } \\
\text { (We did not use the } \\
\text { data from the previous } \\
\text { review as we needed } \\
\text { to be sure about the } \\
\text { studies characteris- } \\
\text { tics) }\end{array}$} & \multirow{21}{*}{$\begin{array}{l}\text { Gerlach 2001: reported on an anticavity whitening } \\
\text { dentifrice; discarded during initial screening } \\
\text { Gerlach 2004a: } 1 \text { of the interventions included a } \\
\text { whitening dentifrice; excluded with reason } \\
\text { Karpinia 2003: } 1 \text { of the interventions included a } \\
\text { whitening dentifrice; excluded with reason } \\
\text { Kowitz 1994a: reported on whitening toothpaste; } \\
\text { discarded during initial screening } \\
\text { Panich 2001: an MSc thesis, did not appear in our } \\
\text { search. We could neither procure the full text of the } \\
\text { thesis nor the published version, hence we did not } \\
\text { include in our review }\end{array}$} \\
\hline Brunton 2004 & Cronin 2005 & & \\
\hline Cronin 2005 & Gerlach 2000 & & \\
\hline Gerlach 2000 & Gerlach 2002 & & \\
\hline Gerlach 2001 & Gerlach 2002a & & \\
\hline Gerlach 2002 & Gerlach 2003 & & \\
\hline Gerlach 2002a & Karpinia 2002 & & \\
\hline Gerlach 2003 & Kihn 2000 & & \\
\hline Gerlach 2004a & Kowitz 1994 & & \\
\hline Gerlach 2004b & Kugel 2000 & & \\
\hline Karpinia 2002 & Li 2003 & & \\
\hline Karpinia 2003 & Li 2004 & & \\
\hline Kihn 2000 & Matis 2000 & & \\
\hline Kowitz 1994 & Mokhlis 2000 & & \\
\hline Kowitz 1994a & Nathoo 1994 & & \\
\hline Kugel 2000 & Nathoo 2002 & & \\
\hline Li 2003 & Nathoo 2003 & & \\
\hline \multicolumn{2}{|l|}{ Li 2004} & & \\
\hline \multicolumn{2}{|l|}{ Matis 2000} & & \\
\hline \multicolumn{2}{|l|}{ Mokhlis 2000} & & \\
\hline Nathoo 1994 & & & \\
\hline
\end{tabular}


Table 2. Comparison of included articles: 2006 review version versus current version (Continued)

Nathoo 2002

Nathoo 2003

Panich 2001

\section{AP PE N DICES}

\section{Appendix 1. Cochrane Oral Health's Trials Register search strategy}

1 (((tooth or teeth or dental) and (whiten* or bleach*)):ti,ab) AND (INREGISTER)

2 (((tooth or teeth or dental) and (stain* and remov $\left.\left.{ }^{\star}\right)\right):$ ti,ab) AND (INREGISTER)

3 (\#1 or \#2) AND (INREGISTER)

4 (("tooth whitening system" or "tooth bleaching system"):ti,ab) AND (INREGISTER)

5 ((tray* and (whiten ${ }^{\star}$ or bleach* or peroxide)):ti,ab) AND (INREGISTER)

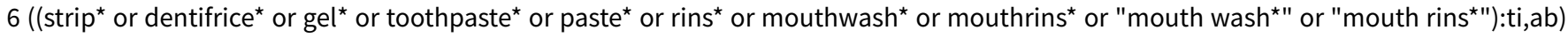
AND (INREGISTER)

7 ("whitening kit*":ti,ab) AND (INREGISTER)

8 (\#4 or \#5 or \#6 or 7 ) AND (INREGISTER)

9 (\#3 and \#8) AND (INREGISTER)

\section{Appendix 2. Cochrane Central Register of Controlled Trials (CENTRAL) search strategy}

\#1 [mh "tooth bleaching"]

\#2 ((tooth or teeth or dental) near/5 (whiten* or bleach* or (stain* near/3 remov*))):ti,ab

\#3 \#1 or \#2

\#4 ("tooth whitening system" or "tooth bleaching system"):ti,ab

\#5 (tray* and (whiten* or bleach* or peroxide)):ti,ab

\#6 [mh dentifrices]

\#7 ((strip* or dentifrice* or gel ${ }^{\star}$ or toothpaste* or paste* or rins* or mouthwash* or mouthrins ${ }^{\star}$ or "mouth wash" or "mouth rins" (whiten ${ }^{\star}$ or bleach* or peroxide)):ti,ab

\#8 "whitening kit*":ti,ab

$\# 9$ \{or \#4-\#8\}

$\# 10 \# 3$ and \#9

\section{Appendix 3. MEDLINE Ovid search strategy}

1. Tooth bleaching/

2. ((tooth or teeth or dental) adj5 (whiten\$ or bleach\$ or (stain\$ adj3 remov\$))).ti,ab.

3. 1 or 2

4. ("tooth whitening system" or "tooth bleaching system").ti,ab.

5. (tray\$ and (whiten\$ or bleach\$ or peroxide)).ti,ab.

6. Dentifrices/

7. ((strip\$ or dentifrice\$ or gel\$ or toothpaste\$ or paste\$ or rins\$ or mouthwash\$ or mouthrins\$ or "mouth wash\$" or "mouth rins\$") and (whiten\$ or bleach\$ or peroxide)).ti,ab.

8. "whitening kit\$\$".ti,ab.

9. or/4-8

10. 3 and 9

This subject search was linked to the Cochrane Highly Sensitive Search Strategy (CHSSS) for identifying randomised trials in MEDLINE: sensitivity- maximising version (2008 revision) as referenced in Chapter 6.4.11.1 and detailed in box 6.4.c of theCochrane Handbook for Systematic Reviews of Interventions, Version 5.1.0 (updated March 2011) (Lefebvre 2011).

1. randomised controlled trial.pt.

2. controlled clinical trial.pt.

3. randomized.ab.

4. placebo.ab.

5. drug therapy.fs. 
6. randomly.ab.

7. trial.ab.

8. groups.ab.

9. or/1-8

10. exp animals/ not humans.sh.

11.9 not 10

\section{Appendix 4. Embase Ovid search strategy}

1. tooth discoloration/

2. ((tooth or teeth or dental) adj5 (whiten\$ or bleach\$ or (stain\$ adj3 remov\$))).ti,ab.

3.1 or 2

4. tooth bleaching agent/

5. ("tooth whitening system" or "tooth bleaching system").ti,ab.

6. (tray\$ and (whiten\$ or bleach\$ or peroxide)).ti,ab.

7. toothpaste/

8. (strip\$ or dentifrice or gel\$ or toothpaste\$ or paste\$ or rins\$ or mouthwash\$ or mouthrins\$ or "mouth wash\$" or "mouth rins\$").ti,ab.

9. "whitening kit\$".ti,ab.

10. or/4-9

11.3 and 10

This subject search was linked to an adapted version of the Cochrane Embase Project filter for identifying randomised controlled trials in Embase Ovid (see http://www.cochranelibrary.com/help/central-creation-details.html for information).

1. Randomized controlled trial/

2. Controlled clinical study/

3. Random\$.ti,ab.

4. randomization/

5. intermethod comparison/

6. placebo.ti,ab.

7. (compare or compared or comparison).ti.

8. ((evaluated or evaluate or evaluating or assessed or assess) and (compare or compared or comparing or comparison)).ab.

9. (open adj label).ti,ab.

10. ((double or single or doubly or singly) adj (blind or blinded or blindly)).ti,ab.

11. double blind procedure/

12. parallel group $\$ 1 . t i, a b$.

13. (crossover or cross over).ti,ab.

14. ((assign\$ or match or matched or allocation) adj5 (alternate or group $\$ 1$ or intervention $\$ 1$ or patient $\$ 1$ or subject $\$ 1$ or participant

\$1)).ti,ab.

15. (assigned or allocated).ti,ab.

16. (controlled adj7 (study or design or trial)).ti,ab.

17. (volunteer or volunteers).ti,ab.

18. trial.ti.

19. or/1-18

20. (exp animal/ or animal.hw. or nonhuman/) not (exp human/ or human cell/ or (human or humans).ti.)

21. 19 not 20

\section{Appendix 5. US National Institutes of Health Ongoing Trials Register ClinicalTrials.gov search strategy}

whiten and teeth

bleach and teeth

tooth and stain and removal

\section{Appendix 6. World Health Organization International Clinical Trials Registry Platform search strategy}

whiten and tooth or whiten and teeth

bleach and tooth or bleach and teeth

tooth and stain and removal

\section{WHAT'S NEW}




\begin{tabular}{lll}
\hline Date & Event & Description \\
\hline 12 June 2018 & $\begin{array}{l}\text { New citation required and conclusions } \\
\text { have changed }\end{array}$ & $\begin{array}{l}\text { New authors. Review update including 46 new studies bringing } \\
\text { the total to 71 included studies. Methods updated. 'Summary of } \\
\text { findings' tables included. Conclusions changed. }\end{array}$ \\
\hline
\end{tabular}

12 June $2018 \quad$ New search has been performed

Searches updated to 12 June 2018.

\section{CONTRIBUTIONS OF AUTHORS}

- Prashanti Eachempati: protocol, selecting trials, analyses, final review, and updating review.

- Sumanth Kumbargere Nagraj: arbiter, analyses, final review, and updating review.

- Salian Kiran Kumar Krishanappa: obtaining copies of trials, selecting trials, data extraction, and entering data into Review Manager 5.

- Puneet Gupta: selecting trials, data extraction, and entering data into Review Manager 5.

- Ibrahim Ethem Yayali: selecting trials, data extraction, and entering data into Review Manager 5.

\section{DECLARATIONS OF INTEREST}

Prashanti Eachempati: none known.

Sumanth Kumbargere Nagraj: none known.

Salian Kiran Kumar Krishanappa: none known.

Puneet Gupta: none known.

Ibrahim Ethem Yaylali: none known.

\section{SOURCES OF SUPPORT}

\section{Internal sources}

- Faculty of Dentistry, Melaka-Manipal Medical College, Manipal University, Melaka Campus, Malaysia.

\section{External sources}

- National Institute for Health Research (NIHR), UK.

This project was supported by the NIHR, via Cochrane Infrastructure funding to Cochrane Oral Health. The views and opinions expressed herein are those of the review authors and do not necessarily reflect those of the Systematic Reviews Programme, the NIHR, the NHS or the Department of Health.

- Cochrane Oral Health Global Alliance, Other.

The production of Cochrane Oral Health reviews has been supported financially by our Global Alliance since 2011 (oralhealth.cochrane.org/partnerships-alliances). Contributors over the past year have been the American Association of Public Health Dentistry, USA; AS-Akademie, Germany; the British Association for the Study of Community Dentistry, UK; the British Society of Paediatric Dentistry, UK; the Canadian Dental Hygienists Association, Canada; the Centre for Dental Education and Research at All India Institute of Medical Sciences, India; the National Center for Dental Hygiene Research \& Practice, USA; New York University College of Dentistry, USA; NHS Education for Scotland, UK; and the Swiss Society for Endodontology, Switzerland.

\section{DIFFERENCES BETWEEN PROTOCOL AND REVIEW}

There are a few differences between the previous version of the review and this updated version.

- Previous review considered outcome data for tooth whiteness immediately after 2 weeks of product applications whereas we considered trials with any duration of treatment.

- This version excluded quasi-randomised trials.

- Trials addressing bleaching for tetracycline-stained teeth were not included in the previous review. We included two trials comparing the effects of a bleaching agent on participants with tetracycline stains.

- Some trials which were included in the previous review are not included in the present review as they consisted of whitening dentifrices which were part of our exclusion criteria (Additional Table 2).

- Trials on whitening chewing gums and mouthrinses are included in our current review but absent in the previous review.

- In the previous review analysis was not combined for trials with the same bleaching agent using different measurement methods (Vita shade guide and colorimeter). We used standardised mean difference (SMD) and combined data wherever relevant. 
- Report on examiner calibration and correlation analysis between concentration of peroxide and effect size was not included in our review but is mentioned in the previous review.

- We updated the methods, used GRADE to assess the certainty of the evidence and included 'Summary of findings' tables.

\section{N D EX TERM S}

\section{Medical Subject Headings (MeSH)}

Carbamide Peroxide [adverse effects] [therapeutic use]; Chewing Gum; Hydrogen Peroxide [adverse effects] [therapeutic use]; Mouthwashes [therapeutic use]; Nonprescription Drugs; Phosphates [therapeutic use]; Polyphosphates [therapeutic use]; Randomized Controlled Trials as Topic; Self Care [ ${ }^{*}$ methods]; Tooth Bleaching [adverse effects] [ ${ }^{*}$ methods]; Tooth Bleaching Agents [adverse effects] [ ${ }^{\star}$ therapeutic use]; Toothpastes [therapeutic use]; Urea [therapeutic use]

\section{MeSH check words}

Adult; Humans 